Review

\title{
Calcium Orthophosphate-Containing Biocomposites and Hybrid Biomaterials for Biomedical Applications
}

\author{
Sergey V. Dorozhkin \\ Kudrinskaja sq. 1-155, Moscow 123242, Russia; E-Mail: sedorozhkin@yandex.ru \\ Academic Editor: Francesco Puoci
}

Received: 16 June 2015 / Accepted: 1 August 2015 / Published: 7 August 2015

\begin{abstract}
The state-of-the-art on calcium orthophosphate $\left(\mathrm{CaPO}_{4}\right)$-containing biocomposites and hybrid biomaterials suitable for biomedical applications is presented. Since these types of biomaterials offer many significant and exciting possibilities for hard tissue regeneration, this subject belongs to a rapidly expanding area of biomedical research. Through the successful combinations of the desired properties of matrix materials with those of fillers (in such systems, $\mathrm{CaPO}_{4}$ might play either role), innovative bone graft biomaterials can be designed. Various types of $\mathrm{CaPO}_{4}$-based biocomposites and hybrid biomaterials those are either already in use or being investigated for biomedical applications are extensively discussed. Many different formulations in terms of the material constituents, fabrication technologies, structural and bioactive properties, as well as both in vitro and in vivo characteristics have been already proposed. Among the others, the nano-structurally controlled biocomposites, those containing nanodimensional compounds, biomimetically fabricated formulations with collagen, chitin and/or gelatin, as well as various functionally graded structures seem to be the most promising candidates for clinical applications. The specific advantages of using $\mathrm{CaPO}_{4}$-based biocomposites and hybrid biomaterials in the selected applications are highlighted. As the way from a laboratory to a hospital is a long one and the prospective biomedical candidates have to meet many different necessities, the critical issues and scientific challenges that require further research and development are also examined.
\end{abstract}

Keywords: calcium orthophosphates; hydroxyapatite; biocomposites; hybrid biomaterials; bone grafts; biomedical applications; tissue engineering 


\section{Introduction}

The fracture of bones due to various traumas or natural aging is a typical type of a tissue failure. An operative treatment frequently requires implantation of a temporary or a permanent prosthesis, which still is a challenge for orthopedic surgeons, especially in the cases of large bone defects. A fast aging of the population and serious drawbacks of natural bone grafts make the situation even worse; therefore, there is a high clinical demand for bone substitutes. Unfortunately, a medical application of xenografts (e.g., bovine bone) is generally associated with potential viral infections. In addition, xenografts have a low osteogenicity, an increased immunogenicity and, usually, resorb more rapidly than autogenous bone. Similar limitations are also valid for human allografts (i.e., tissue transplantation between individuals of the same species but of non-identical genetic composition), where the concerns about potential risks of transmitting tumor cells, a variety of bacterial and viral infections, as well as immunological and blood group incompatibility are even stronger. Moreover, harvesting and conservation of allografts (exogenous bones) are additional limiting factors [1-3]. Autografts (endogenous bones) are still the "golden standard" among any substitution materials because they are osteogenic, osteoinductive, osteoconductive, completely biocompatible, non-toxic and do not cause any immunological problems (non-allergic). They contain viable osteogenic cells, bone matrix proteins and support bone growth. Usually, autografts are well accepted by the body and rapidly integrated into the surrounding bone tissues. Due to these reasons, they are used routinely for a long period with good clinical results [2-5]; however, it is fair to say on complication cases, those frequently happened in the past [6]. Unfortunately, a limited number of donor sites restrict the quantity of autografts harvested from the iliac crest or other locations of the patient's own body. In addition, their medical application is always associated with additional traumas and scars resulting from the extraction of a donor tissue during a superfluous surgical operation, which requires further healing at the donation site and can involve long-term postoperative pain. Thus, any types of biologically derived transplants appear to be imperfect solutions, mainly due to a restricted quantity of donor tissues, donor site morbidity, as well as potential risks of an immunological incompatibility and disease transfer [7-9]. In this light, manmade materials (alloplastic or synthetic bone grafts) stand out as a reasonable option because they are easily available, might be processed and modified to suit the specific needs of a given application. What is more, there are no concerns about potential infections, immunological incompatibility, sterility and donor site morbidity. Therefore, investigations on artificial materials for bone tissue repair appear to be one of the key subjects in the field of biomaterials research for clinical applications $[10,11]$.

Currently, there are several classes of synthetic bone grafting biomaterials for in vivo applications. The examples include natural coral, coral-derived materials, bovine porous demineralized bone, human demineralized bone matrix, bioactive glasses, glass-ceramics and $\mathrm{CaPO}_{4}[12,13]$. Among them, porous bioceramics made of $\mathrm{CaPO}_{4}$ appear to be very prominent due to both the excellent biocompatibility and bonding ability to living bone in the body. This is directly related to the fact that the inorganic material of mammalian calcified tissues, i.e., of bone and teeth, consists of $\mathrm{CaPO}_{4}[14,15]$. Due to this reason, other artificial materials are normally encapsulated by fibrous tissue, when implanted in body defects, while $\mathrm{CaPO}_{4}$ are not. Many types of $\mathrm{CaPO}_{4}$-based bioceramics with different chemical composition are already on the market $[16,17]$. Unfortunately, as for any ceramic material, $\mathrm{CaPO}_{4}$ bioceramics alone lack the mechanical and elastic properties of the calcified tissues. Namely, scaffolds made of $\mathrm{CaPO}_{4}$ only 
suffer from a low elasticity, a high brittleness, a poor tensile strength, a low mechanical reliability and fracture toughness, which leads to various concerns about their mechanical performance after implantation. Besides, in many cases, it is difficult to form $\mathrm{CaPO}_{4}$ bioceramics into the desired shapes [16,17].

The superior strength and partial elasticity of biological calcified tissues (e.g., bones) are due to the presence of bioorganic polymers (mainly, collagen type I fibers) rather than to a natural ceramic (mainly, a poorly crystalline ion-substituted calcium-deficient hydroxyapatite (CDHA), often referred to as "biological apatite") phase [18-21]. The elastic collagen fibers are aligned in bone along the main stress directions. The biochemical composition of bones is given in Table 1 [22]. A decalcified bone becomes very flexible being easily twisted, whereas a bone without collagen is very brittle; thus, the inorganic nano-sized crystals of biological apatite provide with the hardness and stiffness, while the bioorganic fibers are responsible for the elasticity and toughness. In bones, both types of materials integrate each other into a nanometric scale in such a way that the crystallite size, fibers orientation, short-range order between the components, etc., determine its nanostructure and therefore the function and mechanical properties of the entire composite. From the mechanical point of view, bone is a tough material at low strain rates but fractures more like a brittle material at high strain rates; generally, it is rather weak in tension and shear, particularly along the longitudinal plane. Besides, bone is an anisotropic material because its properties are directionally dependent [18-21].

Table 1. The biochemical composition of bones [22]. The composition is varied from species to species and from bone to bone.

\begin{tabular}{c|c|c|c}
\hline Inorganic Phases & wt \% & Bioorganic Phases & wt \% \\
\hline $\mathrm{CaPO}_{4}$ (biological apatite) & $\sim 60$ & collagen type I & $\sim 20$ \\
\hline water & $\sim 9$ & $\begin{array}{c}\text { non-collagenous proteins: } \\
\text { osteocalcin, osteonectin, } \\
\text { osteopontin, thrombospondin, } \\
\text { morphogenetic proteins, } \\
\text { sialoprotein, serum proteins }\end{array}$ & $\sim 3$ \\
\hline carbonates & $\sim 4$ & $\begin{array}{c}\text { other traces: polysaccharides, } \\
\text { lipids, cytokines }\end{array}$ & balance \\
\hline citrates & $\sim 0.9$ & $\begin{array}{c}\text { primary bone cells: osteoblasts, } \\
\text { osteocytes, osteoclasts }\end{array}$ & balance \\
\hline sodium & $\sim 0.7$ & & \\
\hline magnesium & $\sim 0.5$ & & \\
\hline other traces: $\mathrm{Cl}^{-}, \mathrm{F}^{-}, \mathrm{K}^{+} \mathrm{Sr}^{2+}, \mathrm{Pb}^{2+}, \mathrm{Zn}^{2+}, \mathrm{Cu}^{2+}, \mathrm{Fe}^{2+}$ & balance & & \\
\hline
\end{tabular}

It remains a great challenge to design the ideal bone graft that emulates the nature's own structures or functions. Certainly, the successful design requires an appreciation of the bones' structure. According to expectations, the ideal bone graft should be benign, available in a variety of forms and sizes, all with sufficient mechanical properties for use in load-bearing sites, form a chemical bond at the bone/implant interface, as well as be osteogenic, osteoinductive, osteoconductive, biocompatible, completely biodegradable at the expense of bone growth and moldable to fill and restore bone defects [23,24]. Further, it should resemble the chemical composition of bones (thus, the presence of $\mathrm{CaPO}_{4}$ is mandatory), exhibit contiguous porosity to encourage invasion by the live host tissue, as well as possess 
both viscoelastic and semi-brittle behavior, as bones do [25-27]. Moreover, the degradation kinetics of the ideal implant should be adjusted to the healing rate of the human tissue with absence of any chemical or biological irritation and/or toxicity caused by substances, which are released due to corrosion or degradation. Ideally, the combined mechanical strength of the implant and the ingrowing bone should remain constant throughout the regenerative process. Furthermore, the substitution implant material should not disturb significantly the stress environment of the surrounding living tissue [28]. Finally, there is an opinion, that in the case of a serious trauma, bone should fracture rather than the implant [23]. A good sterilizability, storability and processability, as well as a relatively low cost are also of a great importance to permit a clinical application. Unfortunately, no artificial biomaterial is yet available, which embodies all these requirements and unlikely it will appear in the nearest future. To date, most of the available biomaterials appear to be either predominantly osteogenic or osteoinductive or else purely osteoconductive [1].

Careful consideration of the bone type and mechanical properties are needed to design bone substitutes. Indeed, in high load-bearing bones such as the femur, the stiffness of the implant needs to be adequate, not too stiff to result in strain shielding, but rigid enough to present stability. However, in relatively low load-bearing applications such as cranial bone repairs, it is more important to have stability and the correct three-dimensional shapes for aesthetic reasons. One of the most promising alternatives is to apply materials with similar composition and nanostructure to that of bone tissue. Mimicking the structure of calcified tissues and addressing the limitations of the individual materials, development of organic-inorganic hybrid biomaterials provides excellent possibilities for improving the conventional bone implants. In this sense, suitable biocomposites of tailored physical, biological and mechanical properties with the predictable degradation behavior can be prepared combining biologically relevant $\mathrm{CaPO}_{4}$ with bioresorbable polymers [29]. As a rule, the general behavior of such biocomposites is dependent on nature, structure and relative contents of the constitutive components, although other parameters such as the preparation conditions also determine the properties of the final materials. Currently, $\mathrm{CaPO}_{4}$ is incorporated as either a filler or a coating (or both) either into or onto a biodegradable polymer matrix, in the form of particles or fibers, and are increasingly considered for using as bone tissue engineering scaffolds due to their improved physical, biologic and mechanical properties [30-33]. In addition, such biocomposites could fulfill general requirements to the next generation of biomaterials, those should combine the bioactive and bioresorbable properties to activate in vivo mechanisms of tissue regeneration, stimulating the body to heal itself and leading to replacement of the implants by the regenerating tissue. Thus, through the successful combinations of ductile polymer matrixes with hard and bioactive particulate bioceramic fillers, optimal materials can be designed and, ideally, this approach could lead to a superior construction to be used as either implants or posterior dental restorative material $[29,34]$.

A lint-reinforced plaster was the first composite used in clinical orthopedics as an external immobilizer (bandage) in the treatment of bone fracture by Mathijsen in 1852 [35], followed by Dreesman in 1892 [36]. A great progress in the clinical application of various types of composite materials has been achieved since then. Based on both the past experience and the newly gained knowledge, various composite materials with tailored mechanical and biological performance can be manufactured and used to meet various clinical requirements [37]. However, this review presents only a brief history and advances in the field of $\mathrm{CaPO}_{4}$-based biocomposites and hybrid biomaterials suitable 
for biomedical application. The majority of the reviewed literature is restricted to the recent publications; a limited number of papers published in the XX-th century have been cited. Various aspects of the material constituents, fabrication technologies, structural and bioactive properties, as well as phase interaction have been considered and discussed in details. Finally, several critical issues and scientific challenges that are needed for further advancement are outlined.

\section{General Information and Knowledge}

According to Wikipedia, the free encyclopedia, "composite materials (or composites for short) are engineered materials made from two or more constituent materials with significantly different physical or chemical properties and which remain separate and distinct on a macroscopic level within the finished structure" [38]. Thus, composites are always heterogeneous. Furthermore, the phases of any composite retain their identities and properties, and are bonded, which is why an interface is maintained between them. This provides improved specific or synergistic characteristics that are not obtainable by any of the original phases alone [39]. Following the point of view of some predecessors, we also consider that "for the purpose of this review, composites are defined as those having a distinct phase distributed through their bulk, as opposed to modular or coated components" [40] (p. 1329). For this reason, with a few important exceptions, the structures obtained by soaking of various materials in supersaturated solutions containing ions of calcium and orthophosphate (e.g., Refs. [41-44]), those obtained by coating of various materials by $\mathrm{CaPO}_{4}$ (reviewed in Refs. [45-47]), as well as $\mathrm{CaPO}_{4}$ coated by other compounds [48-51] have not been considered; however, composite coatings have been considered. Occasionally, porous $\mathrm{CaPO}_{4}$ scaffolds filled by cells inside the pores [52-55], as well as $\mathrm{CaPO}_{4}$ impregnated by biologically active substances [56,57] are also defined as composites and/or hybrids; nevertheless, such structures have not been considered either.

In any composite, there are two major categories of constituent materials: a matrix (or a continuous phase) and (a) dispersed phase(s). To create a composite, at least one portion of each type is required. General information on the major fabrication and processing techniques might be found elsewhere [40,58]. The continuous phase is responsible for filling the volume, as well as it surrounds and supports the dispersed material(s) by maintaining their relative positions. The dispersed phase(s) is (are) usually responsible for enhancing one or more properties of the matrix. Most of the composites target an enhancement of mechanical properties of the matrix, such as stiffness and strength; however, other properties, such as erosion stability, transport properties (electrical or thermal), radiopacity, density or biocompatibility might also be of a great interest. This synergism produces the properties, which are unavailable from the individual constituent materials [58,59]. What's more, by controlling the volume fractions and local and global arrangement of the dispersed phase, the properties and design of composites can be varied and tailored to suit the necessary conditions. For example, in the case of ceramics, the dispersed phase serves to impede crack growth. In this case, it acts as reinforcement. A number of methods, including deflecting crack tips, forming bridges across crack faces, absorbing energy during pullout and causing a redistribution of stresses in regions, adjacent to crack tips, can be used to accomplish this [60]. Other factors to be considered in composites are the volume fraction of the dispersed phase(s), its (their) orientation and homogeneity of the overall composite. For example, higher volume fractions of reinforcement phases tend to improve the mechanical properties of the composites, 
while continuous and aligned fibers best prevent crack propagation with the added property of anisotropic behavior. From a structural point of view, composites are anisotropic in nature: their mechanical properties are different in different directions. Furthermore, the uniform distribution of the dispersed phase is also desirable, as it imparts consistent properties to the composite $[38,58,59]$.

In general, composites might be simple, complex, graded and hierarchical. The term "a simple composite" is referred to the composites those result from the homogeneous dispersion of one dispersed phase throughout a matrix. The term "a complex composite" is referred to the composites those result from the homogeneous dispersion of several dispersed phases throughout one matrix. The term "a graded composite" is referred to the composites those result from the intentionally structurally inhomogeneous dispersion of one or several dispersed phases throughout one matrix. The term "a hierarchical composite" is referred to the cases, when fine entities of either a simple or a complex composite is somehow aggregated to form coarser ones (e.g., granules or particles) which afterwards are dispersed inside another matrix to produce the second hierarchical scale of the composite structure. There is another set of four types of composites: (i) fibrous composites, where the fibers are in a matrix; (ii) laminar composites, in which the phases are in layers; (iii) particulate composites, where the particles or flakes are in a matrix; and (iv) hybrid composites, which are combinations of any of the above. Still other classification type of the available composites is based on the matrix materials (metals, ceramics and polymers) [37].

In most cases, three interdependent factors must be considered in designing of any composite: (i) a selection of the suitable matrix and dispersed materials, (ii) a choice of appropriate fabrication and processing methods, (iii) both internal and external design of the device itself [40]. Furthermore, any composite must be formed to shape. To do this, the matrix material can be added before or after the dispersed material has been placed into a mold cavity or onto the mold surface. The matrix material experiences a melding event, that, depending upon the nature of the matrix material, can occur in various ways such as chemical polymerization, setting, curing or solidification from a melted state. Due to a general inhomogeneity, the physical properties of many composite materials are not isotropic but rather orthotropic (i.e., there are different properties or strengths in different orthogonal directions) $[38,58,59]$.

In order to prepare any type of a composite, at least, two different materials must be mixed. Thus, a phase miscibility phenomenon appears to be of the paramount importance $[61,62]$. Furthermore, the interfacial strength among the phases is a very important factor because a lack of adhesion among the phases will result in an early failure at the interface and thus in a decrease in the mechanical properties, especially the tensile strength. From a chemical point of view, we can distinguish several types of the interaction among the composite components: materials with strong (covalent, coordination, ionic) interactions; those with weak interactions (van der Waals forces, hydrogen bonds, hydrophilic-hydrophobic balance) or without chemical interactions among the components [63]. Wetting is also important in bonding or adherence of the materials. It depends on the hydrophilicity or polarity of the filler(s) and the available polar groups of the matrix.

Regarding biocomposites, they are defined as nontoxic composites able to interact well with the human body in vivo and, ideally, contain one or more component(s) that stimulate(s) the healing process and uptake of the implant [64]. Thus, for biocomposites the biological compatibility appears to be more important than any other type of compatibility [37,65-67]. Interestingly that according to the databases, the first paper with the term "biocomposite" in the title was published in 1987 [68] and the one containing 
a combination of terms "biocomposite" and HA in the title was published in 1991 [69]. Thus, the subject of $\mathrm{CaPO}_{4}$-based biocomposites and hybrid biomaterials appears to be quite new. The most common properties from the bioorganic and inorganic domains to be combined in biocomposites have been summarized in Table 2 [24]. For general advantages of the modern $\mathrm{CaPO}_{4}$-based biocomposites over $\mathrm{CaPO}_{4}$ bioceramics and bioresorbable polymers individually, the interested readers are advised to get through "Composite materials strategy" section of Ref. [29].

Table 2. General respective properties from the bioorganic and inorganic domains, to be combined in various composites and hybrid materials [24].

\begin{tabular}{cc}
\hline Inorganic & Bioorganic \\
\hline hardness, brittleness & elasticity, plasticity \\
high density & low density \\
thermal stability & permeability \\
hydrophilicity & hydrophobicity \\
high refractive index & selective complexation \\
mixed valence slate (red-ox) & chemical reactivity \\
strength & bioactivity \\
\hline
\end{tabular}

\section{The Major Constituents}

\section{1. $\mathrm{CaPO}_{4}$}

$\mathrm{CaPO}_{4}$ were first mentioned in 1769 as the major constituents of bones and have been investigated since then [70,71]. The main driving force behind the use of $\mathrm{CaPO}_{4}$ as bone substitute materials is their chemical similarity to the mineral component of mammalian bones and teeth [14-16]. As a result, in addition to being non-toxic, they are biocompatible, not recognized as foreign materials in the body and, most importantly, both exhibit bioactive behavior and integrate into living tissue by the same processes active in remodeling healthy bone. This leads to an intimate physicochemical bond between the implants and bone, termed osteointegration. More to the point, $\mathrm{CaPO}_{4}$ are also known to support osteoblast adhesion and proliferation. Even so, the major limitations to use $\mathrm{CaPO}_{4}$ as load-bearing biomaterials are their mechanical properties; namely, they are brittle with poor fatigue resistance [23]. The poor mechanical behavior is even more evident for highly porous ceramics and scaffolds because porosity greater than $100 \mu \mathrm{m}$ is considered as the requirement for proper vascularization and bone cell colonization [72,73]. That is why, in biomedical applications $\mathrm{CaPO}_{4}$ are used primarily as fillers and coatings [16].

The complete list of known $\mathrm{CaPO}_{4}$, including their standard abbreviations and the major properties, is given in Table 3, while the detailed information on $\mathrm{CaPO}_{4}$, might be found in special books and monographs [16,74-78]. 
Table 3. Existing $\mathrm{CaPO}_{4}$ and their major properties [16].

\begin{tabular}{|c|c|c|c|c|c|}
\hline $\begin{array}{c}\mathrm{Ca} / \mathrm{P} \\
\text { Molar } \\
\text { Ratio }\end{array}$ & Compound & Formula & $\begin{array}{c}\text { Solubility at } \\
25^{\circ} \mathrm{C},-\log \left(K_{\mathrm{s}}\right)\end{array}$ & $\begin{array}{l}\text { Solubility at } \\
25^{\circ} \mathrm{C}, \mathrm{g} / \mathrm{L}\end{array}$ & $\begin{array}{c}\text { pH Stability Range in } \\
\text { Aqueous Solutions at } 25^{\circ} \mathrm{C}\end{array}$ \\
\hline 0.5 & Monocalcium phosphate monohydrate (MCPM) & $\mathrm{Ca}\left(\mathrm{H}_{2} \mathrm{PO}_{4}\right)_{2} \cdot \mathrm{H}_{2} \mathrm{O}$ & 1.14 & $\sim 18$ & $0.0-2.0$ \\
\hline 0.5 & Monocalcium phosphate anhydrous (MCPA or MCP) & $\mathrm{Ca}\left(\mathrm{H}_{2} \mathrm{PO}_{4}\right)_{2}$ & 1.14 & $\sim 17$ & [c] \\
\hline 1.0 & $\begin{array}{l}\text { Dicalcium phosphate dihydrate (DCPD), } \\
\text { mineral brushite }\end{array}$ & $\mathrm{CaHPO}_{4} \cdot 2 \mathrm{H}_{2} \mathrm{O}$ & 6.59 & $\sim 0.088$ & $2.0-6.0$ \\
\hline 1.0 & $\begin{array}{l}\text { Dicalcium phosphate anhydrous (DCPA or DCP), } \\
\text { mineral monetite }\end{array}$ & $\mathrm{CaHPO}_{4}$ & 6.90 & $\sim 0.048$ & [c] \\
\hline 1.33 & Octacalcium phosphate (OCP) & $\mathrm{Ca}_{8}\left(\mathrm{HPO}_{4}\right)_{2}\left(\mathrm{PO}_{4}\right)_{4} \cdot 5 \mathrm{H}_{2} \mathrm{O}$ & 96.6 & $\sim 0.0081$ & $5.5-7.0$ \\
\hline 1.5 & $\alpha$-Tricalcium phosphate $(\alpha-$ TCP) & $\alpha-\mathrm{Ca}_{3}\left(\mathrm{PO}_{4}\right)_{2}$ & 25.5 & $\sim 0.0025$ & [a] \\
\hline 1.5 & $\beta$-Tricalcium phosphate $(\beta$-TCP) & $\beta-\mathrm{Ca}_{3}\left(\mathrm{PO}_{4}\right)_{2}$ & 28.9 & $\sim 0.0005$ & [a] \\
\hline $1.2-2.2$ & Amorphous calcium phosphates (ACP) & $\begin{array}{c}\mathrm{Ca}_{x} \mathrm{H}_{y}\left(\mathrm{PO}_{4}\right)_{z} \cdot n \mathrm{H}_{2} \mathrm{O}, n=3-4.5 \\
15 \%-20 \% \mathrm{H}_{2} \mathrm{O}\end{array}$ & [b] & [b] & $\sim 5-12^{[\mathrm{d}]}$ \\
\hline $1.5-1.67$ & $\begin{array}{l}\text { Calcium-deficient hydroxyapatite } \\
\text { (CDHA or Ca-def HA) }{ }^{[e]}\end{array}$ & $\begin{array}{c}\mathrm{Ca}_{10-x}\left(\mathrm{HPO}_{4}\right)_{x}\left(\mathrm{PO}_{4}\right)_{6-x}(\mathrm{OH})_{2-x} \\
(0<x<1)\end{array}$ & $\sim 85$ & $\sim 0.0094$ & $6.5-9.5$ \\
\hline 1.67 & Hydroxyapatite (HA, HAp or OHAp) & $\mathrm{Ca}_{10}\left(\mathrm{PO}_{4}\right)_{6}(\mathrm{OH})_{2}$ & 116.8 & $\sim 0.0003$ & $9.5-12$ \\
\hline 1.67 & Fluorapatite (FA or FAp) & $\mathrm{Ca}_{10}\left(\mathrm{PO}_{4}\right)_{6} \mathrm{~F}_{2}$ & 120.0 & $\sim 0.0002$ & $7-12$ \\
\hline 1.67 & Oxyapatite (OA, OAp or OXA) ${ }^{[f]}$, mineral voelckerite & $\mathrm{Ca}_{10}\left(\mathrm{PO}_{4}\right)_{6} \mathrm{O}$ & $\sim 69$ & $\sim 0.087$ & [a] \\
\hline 2.0 & $\begin{array}{l}\text { Tetracalcium phosphate (TTCP or TetCP), } \\
\text { mineral hilgenstockite }\end{array}$ & $\mathrm{Ca}_{4}\left(\mathrm{PO}_{4}\right)_{2} \mathrm{O}$ & $38-44$ & $\sim 0.0007$ & [a] \\
\hline
\end{tabular}

[a] These compounds cannot be precipitated from aqueous solutions.

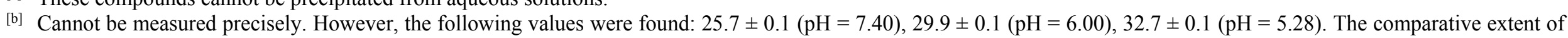
dissolution in acidic buffer is: ACP $>>\alpha-\mathrm{TCP}>>\beta-\mathrm{TCP}>\mathrm{CDHA}>>\mathrm{HA}>\mathrm{FA}$.

[c] Stable at temperatures above $100{ }^{\circ} \mathrm{C}$.

[d] Always metastable.

[e] Occasionally, it is called "precipitated HA (PHA)".

[f] Existence of OA remains questionable. 


\subsection{Polymers}

Polymers are a class of materials consisting of large molecules, often containing many thousands of small units, or monomers, joined together chemically to form one giant chain. In this respect, polymers are comparable with major functional components of the biological environment: lipids, proteins and polysaccharides. They differ from each other in chemical composition, molecular weight, polydispersity, crystallinity, hydrophobicity, solubility and thermal transitions. Besides, their properties can be fine-tuned over a wide range by varying the type of polymer, chain length, as well as by copolymerization or blending of two or more polymers $[79,80]$. Opposite to ceramics, polymers exhibit substantial viscoelastic properties and easily can be fabricated into complex structures, such as sponge-like sheets, gels or complex structures with intricate porous networks and channels [81]. Being X-ray transparent and non-magnetic polymeric materials are fully compatible with the modern diagnostic methods such as computed tomography and magnetic resonance imaging. Unfortunately, most of them are unable to meet the strict demands of the in vivo physiological environment. Namely, the main requirements to polymers suitable for biomedical applications are that they must be biocompatible, not eliciting an excessive or chronic inflammatory response upon implantation and, for those that degrade, that they breakdown into non-toxic products only. Unfortunately, polymers, for the most part, lack rigidity, ductility and ultimate mechanical properties required in load bearing applications. Thus, despite their good biocompatibility, many of the polymeric materials are mainly used for soft tissue replacements (such as skin, blood vessel, cartilage, ligament replacement, etc). Moreover, the sterilization processes (autoclave, ethylene oxide and ${ }^{6 \circ} \mathrm{Co}$ irradiation) may affect the polymer properties [82].

There is a variety of biocompatible polymers suitable for biomedical applications [83-85]. For example, polyacrylates, poly(acrylonitrile-co-vinylchloride) and polylysine have been investigated for cell encapsulation and immunoisolation [86,87]. Polyorthoesters and PCL have been investigated as drug delivery devices, the latter for long-term sustained release because of their slow degradation rates [88]. PCL is a hydrolytic polyester having appropriate resorption period and releases nontoxic byproducts upon degradation [89,90]. PU is in use in engineering of both hard and soft tissues, as well as in nanomedicine [91]. Polymers considered for orthopedic purposes include polyanhydrides, which have also been investigated as delivery devices (due to their rapid and well-defined surface erosion), for bone augmentation or replacement since they can be photopolymerized in situ [88,92,93]. To overcome their poor mechanical properties, they have been co-polymerized with imides or formulated to be crosslinkable in situ [93]. Other polymers, such as polyphosphazenes, can have their properties (e.g., degradation rate) easily modified by varying the nature of their side groups and have been shown to support osteoblast adhesion, which makes them candidate materials for skeletal tissue regeneration [93]. PPF has emerged as a good bone replacement material, exhibiting good mechanical properties (comparable to trabecular bone), possessing the capability to crosslink in vivo through the $\mathrm{C}=\mathrm{C}$ bond and being hydrolytically degradable. It has also been examined as a material for drug delivery devices [88,92-95]. Polycarbonates have been suggested as suitable materials to make scaffolds for bone replacement and have been modified with tyrosine-derived amino acids to render them biodegradable [88,96]. Polydioxanone has been also tested for biomedical applications [97]. PMMA is widely used in orthopedics, as a bone cement for implant fixation, as well as to repair certain fractures and bone defects, for example, osteoporotic vertebral bodies $[98,99]$. However, PMMA sets by a polymerization of toxic 
monomers, which also evolves significant amounts of heat that damages tissues. Moreover, it is neither degradable nor bioactive, does not bond chemically to bones and might generate particulate debris leading to an inflammatory foreign body response [92,100]. A number of other non-degradable polymers applied in orthopedic surgery include PE in its different modifications such as low density PE, HDPE and ultrahigh molecular weight PE (used as the articular surface of total hip replacement implants [101,102]), polyethylene terepthalate and PP, which are applied to repair knee ligaments [103]. Polyactive ${ }^{\mathrm{TM}}$, a block copolymer of PEG and PBT, was also considered for biomedical application [104-106]. Cellulose $[107,108]$ and its esters $[109,110]$ are also popular. Finally yet importantly, polyethylene oxide, PHB and blends thereof have also been tested for biomedical applications [29].

Nonetheless, the most popular synthetic polymers used in medicine are the linear aliphatic poly $(\alpha-$ hydroxyesters) such as PLA, PGA and their copolymers-PLGA (Table 4). These materials have been extensively studied; they appear to be the only synthetic and biodegradable polymers with an extensive FDA approval history $[29,93,111,112]$. They are biocompatible, mostly non-inflammatory, as well as degrade in vivo through hydrolysis and possible enzymatic action into products that are removed from the body by regular metabolic pathways $[88,93,113]$. Besides, they might be used for drug delivery purposes [114]. Poly( $\alpha$-hydroxyesters) have been investigated as scaffolds for replacement and regeneration of a variety of tissues, cell carriers, controlled delivery devices for drugs or proteins (e.g., growth factors), membranes or films, screws, pins and plates for orthopedic applications $[88,93,115,116]$. Additionally, the degradation rate of PLGA can be adjusted by varying the amounts of the two component monomers (Table 4), which in orthopedic applications can be exploited to create materials that degrade in concert with bone ingrowth [117]. Furthermore, PLGA is known to support osteoblast migration and proliferation $[93,118]$, which is a necessity for bone tissue regeneration. Unfortunately, such polymers on their own, though they reduce the effect of stress-shielding, are too weak to be used in load bearing situations and are only recommended in certain clinical indications, such as ankle and elbow fractures [113]. In addition, they exhibit bulk degradation, leading to both a loss in mechanical properties and lowering of the local solution $\mathrm{pH}$ that accelerates further degradation in an autocatalytic manner. As the body is unable to cope with the vast amounts of implant degradation products, this might lead to an inflammatory foreign body response. Finally, poly( $\alpha$-hydroxyesters) do not possess the bioactive and osteoconductive properties [93,119].

Several classifications of the biomedically relevant polymers are possible. For example, some authors distinguish between synthetic polymers like PE, PMMA, PLA, PGA, PCL, etc., and polymers of biological origin, which comprise polysaccharides (starch, alginate, chitin/chitosan [120,121], gellan gum, cellulose, hyaluronic acid and its derivatives), proteins (soy, collagen, gelatin, fibrin, silk) and a variety of biofibers, such as lignocellulosic natural fibers [122,123]. Among them, natural polymers often posses highly organized structures and may contain an extracellular substance, called ligand, which is necessary to bind with cell receptors. However, they always contain various impurities, which should be removed prior use. As synthetic polymers can be produced under the controlled conditions, they in general exhibit predictable and reproducible mechanical and physical properties such as tensile strength, elastic modulus and degradation rate. Control of impurities is a further advantage of synthetic polymers. Other authors differentiate between resorbable or biodegradable (e.g., poly( $\alpha$-hydroxyesters), polysaccharides and proteins) and non-resorbable (e.g., PE, PP, PMMA and cellulose) polymers [123]. Furthermore, polymeric materials can be broadly classified as thermoplastics and thermosets. For 
example, HDPE and PEEK are the examples of thermoplastics, while polydimethylsiloxane and PMMA are the examples of thermosets [82]. The list of synthetic biodegradable polymers used for biomedical application as scaffold materials is available as Table 1 in Ref. [123], while further details on polymers suitable for biomedical applications are available in literature [82,116,124-129] where the interested readers are referred to. Good reviews on the synthesis of different biodegradable polymers [130], as well as on the experimental trends in polymer composites [131] are available elsewhere.

Table 4. Major properties of several FDA (Food and Drug Administration) approved biodegradable polymers [111]. $T_{\mathrm{g}}$, glass transition temperature; $T_{\mathrm{m}}$, melting point.

\begin{tabular}{|c|c|c|c|}
\hline Polymer & $\begin{array}{c}\text { Thermal Properties } \\
\left({ }^{\circ} \mathrm{C}\right) \\
\end{array}$ & $\begin{array}{c}\text { Tensile Modulus } \\
\text { (GPa) } \\
\end{array}$ & $\begin{array}{c}\text { Degradation Time } \\
\text { (Months) }\end{array}$ \\
\hline polyglycolic acid (PGA) & $\begin{array}{c}T_{\mathrm{g}}=35-40 \\
T_{\mathrm{m}}=225-230\end{array}$ & 7.06 & $\begin{array}{c}\text { 6-12 (strength loss } \\
\text { within } 3 \text { weeks) }\end{array}$ \\
\hline L-polylactic acid (LPLA) & $\begin{array}{c}T_{\mathrm{g}}=60-65 \\
T_{\mathrm{m}}=173-178\end{array}$ & 2.7 & $>24$ \\
\hline D,L-polylactic acid (DLPLA) & $\begin{array}{l}T_{\mathrm{g}}=55-60 \\
\text { amorphous }\end{array}$ & 1.9 & $12-16$ \\
\hline $\begin{array}{c}\text { 85/15 D,L-polylactic-co-glycolic acid } \\
\text { (85/15 DLPLGA) }\end{array}$ & $\begin{array}{l}T_{\mathrm{g}}=50-55 \\
\text { amorphous }\end{array}$ & 2.0 & $5-6$ \\
\hline $\begin{array}{c}\text { 75/25 D,L-polylactic-co-glycolic acid } \\
\text { (75/25 DLPLGA) }\end{array}$ & $\begin{array}{l}T_{\mathrm{g}}=50-55 \\
\text { amorphous }\end{array}$ & 2.0 & $4-5$ \\
\hline $\begin{array}{c}\text { 65/35 D,L-polylactic-co-glycolic acid } \\
\text { (65/35 DLPLGA) }\end{array}$ & $\begin{array}{l}T_{\mathrm{g}}=45-50 \\
\text { amorphous }\end{array}$ & 2.0 & $3-4$ \\
\hline $\begin{array}{c}\text { 50/50 D,L-polylactic-co-glycolic acid } \\
\text { (50/50 DLPLGA) }\end{array}$ & $\begin{array}{l}T_{\mathrm{g}}=45-50 \\
\text { amorphous }\end{array}$ & 2.0 & $1-2$ \\
\hline poly(e-caprolactone) (PCL) & $\begin{array}{c}T_{\mathrm{g}}=(-60)-(-65) \\
T_{\mathrm{m}}=58-63\end{array}$ & 0.4 & $>24$ \\
\hline
\end{tabular}

\subsection{Inorganic Materials and Compounds}

\subsubsection{Metals}

Titanium (Ti) is one of the best biocompatible metals and used most widely as implant $[132,133]$. Besides, there are other metallic implants made of pure $\mathrm{Zr}, \mathrm{Hf}, \mathrm{V}, \mathrm{Nb}$, Ta, Re, $\mathrm{Ni}, \mathrm{Fe}, \mathrm{Cu}, \mathrm{Ag}$, stainless steels and various alloys suitable for biomedical application [134-136]. Recent studies revealed even a greater biomedical potential of porous metals [137-139]. The metallic implants provide the necessary strength and toughness that are required in load-bearing parts of the body and, due to these advantages, metals will continue to play an important role as orthopedic biomaterials in the future, even though there are concerns with regard to the release of certain ions from and corrosion products of metallic implants. Of course, neither metals nor alloys are biomimetic (the term biomimetic can be defined as a processing technique that either mimics or inspires the biological mechanism, in part or whole [140]) in terms of chemical composition because there are no elemental metals in the human body. In addition, even biocompatible metals are bioinert: while not rejected by the human body, any metallic implants cannot actively interact with the surrounding tissues. Nevertheless, in some cases (especially when they are 
coated by $\mathrm{CaPO}_{4}$ [47]) the metallic implants can show a reasonable biocompatibility [141]. Until recently, only permanent implants were made of metals and alloys, in which degradation or corrosion was not desirable. However, during recent years a number of magnesium implants have been proposed which are aimed to degrade in the body in order to make room for ingrowing bones $[142,143]$.

\subsubsection{Glasses and Glass-Ceramics}

Special types of both glasses [144,145] and glass-ceramics [146,147] are also suitable materials for biomedical applications and a special $\mathrm{Na}_{2} \mathrm{O}-\mathrm{CaO}-\mathrm{SiO}_{2}-\mathrm{P}_{2} \mathrm{O}_{5}$ formulation named Bioglass ${ }^{\circledR}$ [148-150] is the most popular among them. They are produced via standard glass production techniques and require pure raw materials. Bioglass ${ }^{\circledR}$ is a biocompatible and osteoconductive biomaterial. It bonds to bone without an intervening fibrous connective tissue interface and, due to these properties, it has been widely used for filling bone defects. The primary shortcoming of Bioglass ${ }^{\circledR}$ is mechanical weakness and low fracture toughness due to an amorphous two-dimensional glass network. The bending strength of most Bioglass ${ }^{\circledR}$ compositions is in the range of $40-60 \mathrm{MPa}$, which is not suitable for major load-bearing applications. Making porosity in Bioglass ${ }^{\circledR}$-based scaffolds is beneficial for even better resorption and bioactivity [148-150].

By heat treatment, a suitable glass can be converted into glass-crystal composites containing crystalline phase(s) of controlled sizes and contents. The resultant glass-ceramics can have superior mechanical properties to the parent glass as well as to sintered crystalline ceramics [146,147]. The bioactive A-W glass-ceramics is made from the parent glass in the pseudoternary system $3 \mathrm{CaO}_{2} \cdot \mathrm{P}_{2} \mathrm{O}_{5}-$ $\mathrm{CaO} \cdot \mathrm{SiO}_{2}-\mathrm{MgO} \cdot \mathrm{CaO} \cdot 2 \mathrm{SiO}_{2}$, which is produced by a conventional melt-quenching method. The bioactivity of A-W glass-ceramics is much higher than that of sintered HA. It possesses excellent mechanical properties and has therefore been used clinically for iliac and vertebrae prostheses and as intervertebral spacers $[151,152]$.

\subsubsection{Ceramics}

Metal oxide ceramics, such as alumina $\left(\mathrm{Al}_{2} \mathrm{O}_{3}\right.$, high purity, polycrystalline, fine grained) zirconia $\left(\mathrm{ZrO}_{2}\right)$ and some other oxides (e.g., $\left.\mathrm{TiO}_{2}, \mathrm{SiO}_{2}\right)$ have been widely studied due to their bioinertness, excellent tribological properties, high wear resistance, fracture toughness and strength, as well as a relatively low friction $[148,153,154]$. Among them, due to transformation from the tetragonal to the monoclinic phase, a volume change occurs when pure zirconia is cooled down, which causes cracking of the zirconia ceramics [155]. Therefore, additives such as calcia $(\mathrm{CaO})$, magnesia $(\mathrm{MgO})$ and yttria $\left(\mathrm{Y}_{2} \mathrm{O}_{3}\right)$ must be mixed with zirconia to stabilize the material in either the tetragonal or the cubic phase. Such material is called PSZ [156,157]. However, the brittle nature of any ceramics has limited their scope of clinical applications and hence more research is needed to improve their properties.

\subsubsection{Carbon}

Due to its bioinertness, excellent tribological properties, fracture toughness and strength, as well as a low friction, elemental carbon has been used as a biomaterial, at least, since 1972 [158]. Applications include orthopedic prostheses, vitreous carbon roots for replacement teeth, structural skeletal extensions, 
bone bridges and hip prostheses. Biomedical properties of amorphous carbon were studied as well [159]. However, current trends represent investigations on biomedical applications of nanodimentional carbon, such as nanotubes $[160,161]$.

Carbon nanotubes with their small dimensions, a high aspect (length to diameter) ratio as well as the exceptional mechanical properties, including extreme flexibility and strength, significant resistance to bending, high resilience and the ability to reverse any buckling of the tube, have the excellent potential to accomplish necessary mechanical properties. The studies revealed that they might possess some bioactivity [162,163]. However, non-functionalized carbon nanotubes tend to agglomerate and form bundles. Besides, they are soluble in neither water nor organic solvents. Luckily, chemical functionalization allows carbon nanotubes to be dispersed more easily, which can improve interfacial bonding with other components of the composites [164]. Furthermore, functionalization of carbon nanotubes with carboxylic groups was found to confer a capacity to induce calcification similar to woven bones [165]. Interestingly that carbon nanotubes might be functionalized by in situ deposition of CDHA on their surface [166].

\section{Biocomposites and Hybrid Biomaterials Containing $\mathrm{CaPO}_{4}$}

Generally, the available $\mathrm{CaPO}_{4}$-containing biocomposites and hybrid biomaterials suitable for biomedical applications might be divided into several (partly overlapping) broad areas:

- biocomposites with polymers;

- $\quad$ self-setting formulations;

- formulations based on nanodimensional $\mathrm{CaPO}_{4}$ and nanodimensional biocomposites;

- biocomposites with collagen;

- formulations with other bioorganic compounds and/or biological macromolecules;

- injectable bone substitutes (IBS);

- biocomposites with inorganic compounds, carbon and metals;

- functionally graded formulations;

- biosensors.

The majority of them were developed following a bone-analogue concept in attempts to mimic natural bones. The details on each area are provided below.

\subsection{Biocomposites with Polymers}

Typically, the polymeric components of biocomposites and hybrid biomaterials comprise polymers that both have shown a good biocompatibility and are routinely used in surgical applications. In general, since polymers have a low modulus (2-7 GPa, as the maximum) as compared to that of bone (3-30 $\mathrm{GPa}), \mathrm{CaPO}_{4}$ bioceramics need to be loaded at a high weight \% ratio. Besides, general knowledge on composite mechanics suggests that any high aspect ratio particles, such as whiskers or fibers, significantly improve the modulus at a lower loading. Thus, some attempts have been already performed to prepare biocomposites containing whisker-like [167-173] or needle-like [174-177] $\mathrm{CaPO}_{4}$, as well as $\mathrm{CaPO}_{4}$ fibers [178]. 
The history of implantable $\mathrm{CaPO}_{4} /$ polymer formulations started in 1981 (however, a more general topic "ceramic-plastic material as a bone substitute" is, at least, 18 years older [179]) from the pioneering study by Prof. William Bonfield and colleagues at Queen Mary College, University of London, performed on HA/PE blends [180,181]. That initial study introduced a bone-analogue concept, when proposed biocomposites comprised a polymer ductile matrix of PE and a ceramic stiff phase of HA, and was substantially extended and developed in further investigations by that research group [66,182-192]. More recent studies included investigations on the influence of surface topography of HA/PE composites on cell proliferation and attachment [193-196]. The material is composed of a particular combination of HA particles at a volume loading of $\sim 40 \%$ uniformly dispensed in a HDPE matrix. The idea was to mimic bones by using a polymeric matrix that can develop a considerable anisotropic character through adequate orientation techniques reinforced with a bone-like bioceramics that assures both a mechanical reinforcement and a bioactive character of the composite. Following FDA approval in 1994, in 1995 this material has become commercially available under the trade-name HAPEX ${ }^{\mathrm{TM}}$ (Smith and Nephew Richards, Bartlett, TN, USA), and to date it has been implanted in over 300,000 patients with the successful results. It remains the only clinically successful bioactive composite, which was a major step in the implant field [148,197]. The major production stages of HAPEX ${ }^{\mathrm{TM}}$ include blending, compounding and centrifugal milling. A bulk material or device is then created from this powder by compression and injection molding [37]. Besides, HA/HDPE biocomposites might be prepared by a hot rolling technique that facilitated uniform dispersion and blending of the reinforcements in the matrix [198]. In addition, PP might be used instead of PE [199-202].

A mechanical interlock between the both phases of HAPEX ${ }^{\mathrm{TM}}$ is formed by shrinkage of HDPE onto the HA particles during cooling $[66,67,203]$. Both HA particle size and their distribution in the HDPE matrix were recognized as important parameters affecting the mechanical behavior of HAPEX ${ }^{\mathrm{TM}}$. Namely, smaller HA particles were found to lead to stiffer composites due to general increasing of interfaces between the polymer and the ceramics; furthermore, rigidity of HAPEX ${ }^{\mathrm{TM}}$ was found to be proportional to HA volume fraction [187]. Furthermore, coupling agents, e.g., 3-trimethoxysiyl propylmethacrylate for HA and acrylic acid for HDPE might be used to improve bonding (by both chemical adhesion and mechanical coupling) between HA and HDPE [204,205]. Obviously, other types of $\mathrm{CaPO}_{4}$ might be used instead of HA in biocomposites with PE [206]. Furthermore, attempts were performed to improve the mechanical properties of HAPEX ${ }^{\mathrm{TM}}$ by incorporating other ceramic phases into the polymer matrix, such as PSZ [207] and alumina [208]. For example, a partial replacement of HA filler particles by PSZ particles was found to lead to an increase in the strength and fracture toughness of HA/HDPE biocomposites. The compressive stress, set up by the volume expansion associated with tetragonal to monoclinic phase transformation of PSZ, inhibits or retards the crack propagation within the composite. This results in an enhanced fracture toughness of the $\mathrm{HA} / \mathrm{ZrO}_{2} / \mathrm{HDPE}$ biocomposite [207].

Various studies revealed that HAPEX ${ }^{\mathrm{TM}}$ attached directly to bones by chemical bonding (a bioactive fixation), rather than forming fibrous encapsulation (a morphological fixation). Initial clinical applications of HAPEX ${ }^{\mathrm{TM}}$ came in orbital reconstruction [209] but since 1995, the main uses of this composite have been in the shafts of middle ear implants for the treatment of conductive hearing loss [210,211]. In both applications, HAPEX ${ }^{\mathrm{TM}}$ offers the advantage of in situ shaping, so a surgeon can make final alterations to optimize the fit of the prosthesis to the bone of a patient and subsequent activity requires only limited mechanical loading with virtually no risk of failure from insufficient tensile strength $[66,67,149]$. As 
compared to cortical bones, HA/PE composites have a superior fracture toughness for HA concentrations below $\sim 40 \%$ and similar fracture toughness in the $45 \%-50 \%$ range. Their Young's modulus is in the range of $1-8 \mathrm{GPa}$, which is quite close to that of bone. The examination of the fracture surfaces revealed that only mechanical bond occurs between HA and PE. Unfortunately, the HA/PE composites are not biodegradable, the available surface area of HA is low and the presence of bioinert PE decreases the ability to bond to bones. Furthermore, HAPEX ${ }^{\mathrm{TM}}$ has been designed with a maximized density to increase its strength but the resulting lack of porosity limits the ingrowth of osteoblasts when the implant is placed into the body [23,150]. Further details on HAPEX ${ }^{\mathrm{TM}}$ are available elsewhere $[66,67]$. Except of HAPEX ${ }^{\mathrm{TM}}$, other types of both HA/PE [212-220] and HA/undisclosed polymer (HA ${ }^{\text {nano }}$ Bone, Promimic AB, Göteborg, Sweden) biocomposites are also known.

Both linear and branched PE was used as a matrix and the biocomposites with the former were found to give a higher modulus [213]. The reinforcing mechanisms in $\mathrm{CaPO}_{4} /$ polymer formulations have yet to be convincingly disclosed. Generally, if a poor filler choice is made, the polymeric matrix might be affected by the filler through reduction of molecular weight during composite processing, formation of an immobilized shell of polymer around the particles (transcrystallization, surface-induced crystallization or epitaxial growth) and changes in conformation of the polymer due to particle surfaces and inter-particle spacing $[66,67]$. On the other hand, the reinforcing effect of $\mathrm{CaPO}_{4}$ particles might depend on the molding technique employed: a higher orientation of the polymeric matrix was found to result in a higher mechanical performance of the composite [218,219].

Many other blends of $\mathrm{CaPO}_{4}$ with various polymers are possible, including rather unusual formulations with dendrimers [221]. Even light-curable $\mathrm{CaPO}_{4} /$ polymer formulations are known [222]. The list of the appropriate $\mathrm{CaPO}_{4}$ is shown in Table 3 (except of MCPM and MCPA - both are too acidic and, therefore, are not biocompatible [16]; nevertheless, to overcome this drawback, they might be mixed with basic compounds, such as $\mathrm{HA}$, TTCP, $\mathrm{CaCO}_{3}, \mathrm{CaO}$, etc.) many biomedically suitable polymers have been listed above. The combination of $\mathrm{CaPO}_{4}$ and polymers into biocomposites has a twofold purpose. The desirable mechanical properties of polymers compensate for a poor mechanical behavior of $\mathrm{CaPO}_{4}$ bioceramics, while in turn the desirable bioactive properties of $\mathrm{CaPO}_{4}$ improve those of polymers, expanding the possible uses of each material within the body [223-226]. Namely, polymers have been added to $\mathrm{CaPO}_{4}$ in order to improve their mechanical strength [223], while $\mathrm{CaPO}_{4}$ fillers have been blended with polymers to improve their compressive strength and modulus, in addition to increasing their osteoconductive properties [119,227-230]. In 1990-s, it was established that with increasing of $\mathrm{CaPO}_{4}$ content, both Young's modulus and bioactivity of the biocomposites generally increased, while the ductility decreased [23]. However, the later investigations revealed that the mechanical properties of $\mathrm{CaPO}_{4} /$ polymer biocomposites were not so straightforward: the strength was found to decrease with increasing the $\mathrm{CaPO}_{4}$ content in such biocomposites [231]. Nevertheless, biocompatibility of such biocomposites is enhanced because $\mathrm{CaPO}_{4}$ fillers induce an increased initial flash spread of serum proteins compared to the more hydrophobic polymer surfaces [232]. What's more, experimental results of these biocomposites indicated favorable cell-material interactions with increased cell activities as compared to each polymer alone [225]. Furthermore, such formulations can provide a sustained release of calcium and orthophosphate ions into the milieus, which is important for mineralized tissue regeneration [224]. Indeed, a combination of two different materials draws on the advantages of each one to create a superior biocomposite with respect to the materials on their own. 
It is logical to assume that the proper biocomposite of a $\mathrm{CaPO}_{4}$ (for instance, $\mathrm{CDHA}$ ) with a bioorganic polymer (for instance, collagen) would yield the physical, chemical and mechanical properties similar to those of human bones. Different ways have been already realized to bring these two components together into biocomposites, like mechanical blending, compounding, ball milling, dispersion of ceramic fillers into a polymer-solvent solution, a melt extrusion of a ceramic/polymer powder mixture, co-precipitation and electrochemical co-deposition [22,37,233-235]. Three methods for preparing a homogeneous blend of HA with PLLA were compared [233]. A dry process, consisting in mixing ceramic powder and polymer pellets before a compression-molding step, was used. The second technique was based on the dispersion of ceramic fillers into a polymer-solvent solution. The third method was a melt extrusion of a ceramic/polymer powder mixture. Mixing dry powders led to a ceramic particle network around the polymer pellets, whereas the solvent and melt methods also produced a homogeneous dispersion of HA in the matrix. The main drawback of the solvent casting method is the risk of potentially toxic organic solvent residues. The melt extrusion method was shown to be a good way to prepare homogeneous ceramic/polymer blends [233].

Besides, there is in situ formation, which involves either synthesizing the reinforcement inside a preformed matrix material or synthesizing the matrix material around the reinforcement [37,236,237]. This is one of the most attractive routes, since it avoids extensive particle agglomeration. For example, several papers have reported in situ formation technique to produce various composites of $\mathrm{CaPO}_{4}$ with carbon nanotubes [238-241]. Other examples comprise using amino acid-capped gold nano-sized particles as scaffolds to grow CDHA [242] and preparation of nano-sized HA/PA biocomposites [243,244]. In certain cases, a mechano-chemical route [245,246], emulsions [247-252], freeze-drying [253,254] and freeze-thawing techniques [255] or gel-templated mineralization [256] might be applied to produce $\mathrm{CaPO}_{4}$-based biocomposites. Various fabrication procedures are well described elsewhere [22,37,233], where the interested readers are referred.

The interfacial bonding between the phases is an important issue of any $\mathrm{CaPO}_{4} / \mathrm{polymer}$ biocomposite. Four types of mutual arrangements of nanodimensional particles to polymer chains have been classified by Kickelbick (Figure 1): (1) inorganic particles embedded in inorganic polymer, (2) incorporation of particles by bonding to the polymer backbone, (3) an interpenetrating network with chemical bonds, (4) an inorganic-organic hybrid polymer [257]. If adhesion among the phases is poor, the mechanical properties of a biocomposite suffer. To solve the problem, various approaches have been already introduced. For example, a diisocyanate coupling agent was used to bind PEG/PBT (Polyactive ${ }^{\mathrm{TM}}$ ) block copolymers to HA filler particles. Using surface-modified HA particles as a filler in a PEG/PBT matrix significantly improved the elastic modulus and strength of the polymer as compared to the polymers filled with ungrafted HA [228,258]. Another group used processing conditions to achieve a better adhesion of the filler to the matrix by pressing blends of varying PLLA and HA content at different temperatures and pressures [259]. The researchers found that maximum compressive strength was achieved at $\sim 15 \mathrm{wt} \%$ of PLLA. By using blends with $20 \mathrm{wt} \%$ of PLLA, the authors also established that increasing the pressing temperature and pressure improved the mechanical properties. The former was explained by decrease in viscosity of the PLLA associated with a temperature increase, hence leading to improved wettability of HA particles. The latter was explained by increased compaction and penetration of pores at higher pressure, in conjunction with a greater fluidity of the polymer at higher temperatures. The combination of high pressures and temperatures was found to decrease porosity and 
guarantee a close apposition of a polymer to the particles, thereby improving the compressive strength [223] and fracture energy [260] of the biocomposites. The PLLA/HA biocomposites scaffolds were found to improve cell survival over plain PLLA scaffolds [261].

It is also possible to introduce porosity into $\mathrm{CaPO}_{4}$-based biocomposites, which is advantageous for most applications as bone substitution material. The porosity facilitates migration of osteoblasts from surrounding bones to the implant site. Various material processing strategies to prepare composite scaffolds with interconnected porosity comprise thermally induced phase separation, solvent casting and particle leaching, solid freeform fabrication techniques, microsphere sintering and coating [123,262-265]. A supercritical gas foaming technique might be used as well [233,266,267].

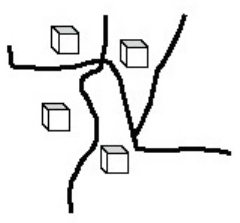

1)

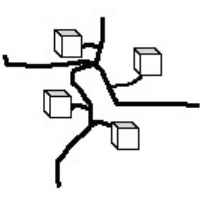

2)

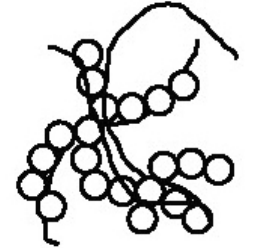

3)

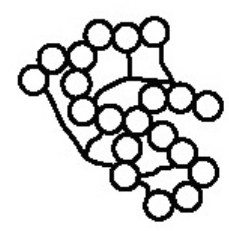

4)

Figure 1. Four types of mutual arrangements of nano-sized particles to a polymer chain: (1) inorganic particles embedded in an inorganic polymer, (2) incorporation of particles by bonding to the polymer backbone, (3) interpenetrating network with chemical bonds, (4) inorganic-organic hybrid polymer. Reprinted from Ref. [257] with permission.

\subsubsection{Apatite-Based Formulations}

A biological apatite is known to be the major inorganic phase of mammalian calcified tissues [14,15]. Consequently, CDHA, HA, carbonateapatite (both with and without dopants) and, occasionally, FA have been applied to prepare biocomposites with other compounds, usually with the aim to improve the bioactivity. For example, polysulfone composed with HA can be used as a starting material for longterm implants [268-270]. Retrieved in vivo, HA/polysulfone biocomposite coated samples from rabbit distal femurs demonstrated direct bone apposition to the coatings, as compared to the fibrous encapsulation that occurred when uncoated samples were used [268]. The resorption time of such biocomposites is a very important factor, which depends on polymer's microstructure and the presence of modifying phases [269].

Various apatite-containing biocomposites with PVA [255,271-275], PVAP [276] and several other polymeric components [277-286] have been already developed. Namely, PVA/CDHA biocomposite blocks were prepared by precipitation of CDHA in aqueous solutions of PVA [255]. An artificial cornea consisted of a porous nano-sized HA/PVA hydrogel skirt and a transparent center of PVA hydrogel has been prepared as well. The results displayed a good biocompatibility and interlocking between artificial cornea and host tissues [271,272]. PVAP has been chosen as a polymer matrix, because its phosphate groups can act as a coupling/anchoring agent, which has a higher affinity toward the HA surface [276]. Greish and Brown developed HA/Ca poly(vinyl phosphonate) biocomposites [280-282]. A templatedriven nucleation and mineral growth process for the high-affinity integration of CDHA with PHEMA hydrogel scaffold has been developed as well [285]. 
PEEK [167,168,287-293] and high impact polystyrene [294,295] were also applied to create biocomposites with HA having a potential for clinical use in load bearing applications. The study on reinforcing PEEK with thermally sprayed HA particles revealed that the mechanical properties increased monotonically with the reinforcement concentration, with a maximum value in the study of $\sim 40 \%$ volume fraction of HA particles [287-289]. The reported ranges of stiffness within 2.8-16.0 GPa and strength within 45.5-69 MPa exceeded the lower values for human bone (7-30 GPa and 50-150 MPa, respectively) [288]. Modeling of the mechanical behavior of HA/PEEK biocomposites is available elsewhere [290].

Biodegradable poly( $\alpha$-hydroxyesters) are well established in clinical medicine. Currently, they provide with a good choice when a suitable polymeric filler material is sought. For example, HA/PLGA formulations were developed which appeared to possess a cellular-compatibility suitable for bone tissue regeneration [296-304]. Zhang and Ma seeded highly porous PLLA foams with HA particles in order to improve the osteoconductivity of polymer scaffolds for bone tissue engineering [227]. They pointed out that hydration of the foams prior to incubation in simulated body fluid increased the amount of carbonated $\mathrm{CDHA}$ material due to an increase of $\mathrm{COOH}$ and $\mathrm{OH}$ groups on the polymer surface, which apparently acted as nucleation sites for apatite. The mechanical properties of $\mathrm{PLA} / \mathrm{CaPO}_{4}$ biocomposites fabricated using different techniques, as well as the results of in vitro and in vivo experiments with them are available in literature [300].

On their own, poly( $\alpha$-hydroxyesters), such as PGA and PLA, are known to degrade to acidic products (glycolic and lactic acids, respectively) that both catalyze polymer degradation and cause inflammatory reactions of the surrounding tissues [305]. Thus, in biocomposites of poly( $\alpha$-hydroxyesters) with $\mathrm{CaPO}_{4}$, the presence of slightly basic compounds (HA, TTCP) to some extent neutralizes the acid molecules, provides with a weak $\mathrm{pH}$-buffering effect at the polymer surface and, therefore, more or less compensates these drawbacks [119,300,306-308]. However, additives of even more basic chemicals (e.g., $\mathrm{CaO}$, $\mathrm{CaCO}_{3}$ ) might be necessary [123,308-310]. Extensive cell culture experiments on pH-stabilized composites of PGA and carbonateapatite were reported, which afterwards were supported by extensive in vitro $\mathrm{pH}$-studies [311]. A consequent development of this approach has led to designing of functionally graded composite skull implants consisting of polylactides, carbonateapatite and $\mathrm{CaCO}_{3}[312,313]$. Besides the pH-buffering effect, inclusion of $\mathrm{CaPO}_{4}$ was found to modify both surface and bulk properties of the biodegradable poly( $\alpha$-hydroxyesters) by increasing the hydrophilicity and water absorption of the polymer matrix, thus altering the scaffold degradation kinetics. For example, polymer biocomposites filled with HA particles was found to hydrolyze homogeneously due to water penetrating into interfacial regions [314].

Biocomposites of poly $\left(\alpha\right.$-hydroxyesters) with $\mathrm{CaPO}_{4}$ are prepared mainly by incorporating the inorganic phase into a polymeric solution, followed by drying under vacuum. The resulting solid biocomposites might be shaped using different processing techniques. One can also prepare these biocomposites by mixing HA particles with L-lactide prior the polymerization [306] or by a combination of slip-casting technique and hot pressing [315]; however, other production techniques are known [300,302,316]. Addition of a surfactant (surface active agent) might be useful to keep the suspension homogeneity [317]. Furthermore, HA/PLA [248,249] and HA/PLGA [250] microspheres might be prepared by a microemulsion technique. More complex formulations, such as carbonated-FA/PLA [318] and PLGA/carbon nanotubes/HA [319], are also known. An interesting list of references, assigned to the 
different ways of preparing $\mathrm{HA} /$ poly( $\alpha$-hydroxyesters) biodegradable composites, might be found in publications by Durucan and Brown [320-322]. The authors prepared CDHA/PLA and CDHA/PLGA biocomposites by solvent casting technique with a subsequent hydrolysis of $\alpha$-TCP to CDHA in aqueous solutions. The presence of both polymers was found to inhibit $\alpha$-TCP hydrolysis, if compared with that of single-phase $\alpha$-TCP; what is more, the inhibiting effect of PLA exceeded that of PLGA [320-322]. The physical interactions between $\mathrm{CaPO}_{4}$ and poly( $\alpha$-hydroxyesters) might be easily seen in Figure 2 [322]. Another set of good pictures might be found in Ref. [51]. Nevertheless, it should not be forgotten that typically non-melt based routes lead to development of composites with lower mechanical performance and many times require the use of toxic solvents and intensive hand labor [125].

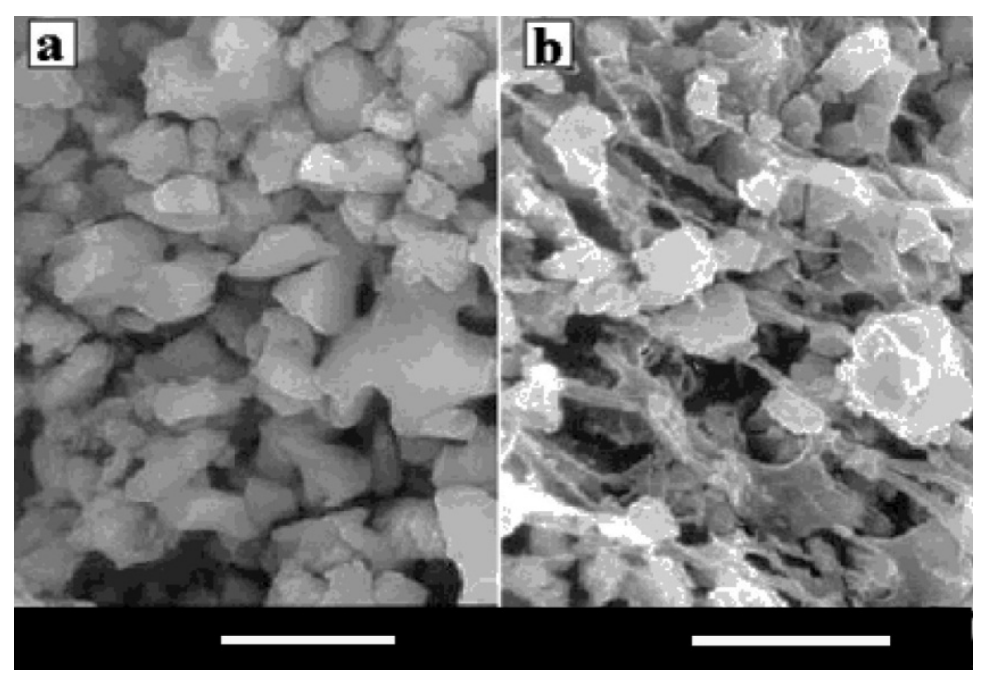

Figure 2. SEM micrographs of (a) $\alpha$-Tricalcium phosphate ( $\alpha$-TCP) compact; (b) $\alpha$-TCP/PLGA biocomposite (bars $=5 \mu \mathrm{m}$ ). Reprinted from Ref. [322] with permission.

The mechanical properties of poly( $\alpha$-hydroxyesters) could be substantially improved by addition of $\mathrm{CaPO}_{4}$ [323,324]. Namely, CDHA/PLLA biocomposites of very high mechanical properties were developed [119] and fixation tools (screws and plates) made of these composites were manufactured and tested. These fixation tools revealed an easy handling and shaping according to the implant site geometry, total resorbability, good ability to bond directly to the bone tissue without interposed fibrous tissue, osteoconductivity, biocompatibility and high stiffness retainable for the period necessary to achieve bone union [314,316]. The initial bending strength of $280 \mathrm{MPa}$ exceeded that of cortical bone (120-210 MPa), while the modulus was as high as $12 \mathrm{GPa}$ [119]. The strength could be maintained above $200 \mathrm{MPa}$ up to 25 weeks in phosphate-buffered saline solution. Such biocomposites could be obtained by precipitation from PLLA/dichloromethane solutions, in which small particles of $\mathrm{CaPO}_{4}$ were distributed [118,325]. Porous scaffolds of PDLLA + HA [267,326,327] and PLGA + HA [328] have been manufactured as well. Upon implantation into rabbit femora, a newly formed bone was observed and biodegradation was significantly enhanced if compared to single-phase HA bioceramics. This might be due to a local release of lactic acid, which in turn dissolves HA. In other studies, PLA and PGA fibers were combined with porous HA scaffolds. Such reinforcement did not hinder bone ingrowth into the implants, which supported further development of such biocomposites as bone graft substitutes [29,300,301]. 
Blends (named as SEVA-C) of EVOH with starch filled with 10-30 wt \% HA have been fabricated to yield biocomposites with modulus up to $\sim 7 \mathrm{GPa}$ with a 30\% HA loading [329-333]. The incorporation of bioactive fillers such as HA into SEVA-C aimed to assure the bioactive behavior of the composite and to provide the necessary stiffness within the typical range of human cortical bone properties. These biocomposites exhibited a strong in vitro bioactivity that was supported by the polymer's water-uptake capability [334]. However, the reinforcement of SEVA-C by HA particles was found to affect the rheological behavior of the blend. A degradation model of these biocomposites has been developed [335].

Higher homologues poly(3-hydroxybutyrate), 3-PHB, and poly(3-hydroxyvalerate) show almost no biodegradation. Nevertheless, biocomposites of these polymers with $\mathrm{CaPO}_{4}$ showed a good biocompatibility both in vitro and in vivo [336-340]. Both bioactivity and mechanical properties of these biocomposites can be tailored by varying the volume percentage of $\mathrm{CaPO}_{4}$. Similarly, biocomposites of PHBHV with both HA and amorphous carbonated apatite (almost ACP) appeared to have a promising potential for repair and replacement of damaged bones [341-344].

Along this line, PCL is used as a slowly biodegradable but well biocompatible polymer. PCL/HA and PCL/CDHA biocomposites have been already discussed as suitable materials for substitution, regeneration and repair of bone tissues [262,345-352]. For example, biocomposites were obtained by infiltration of $\varepsilon$-caprolactone monomer into porous apatite blocks and in situ polymerization [346]. The composites were found to be biodegradable and might be applied as cancellous or trabecular bone replacement material or for a cartilage regeneration. Both the mechanical performance and biocompatibility in osteoblast cell culture of PCL were shown to be strongly increased when HA was added [353]. Several preparation techniques of PCL/HA biocomposites are known [262,349]. For example, to make biocomposite fibers of PCL with nanodimensional HA, the desired amount of nanodimensional HA powder was dispersed in a solvent using magnetic stirrer followed by ultrasonication for $30 \mathrm{~min}$. Then, PCL was dissolved in this suspension, followed by the solvent evaporation [354]. The opposite preparation order is also possible: PCL was initially dissolved in chloroform at room temperature $(7 \%$ $10 \% \mathrm{w} / \mathrm{v})$, then HA $(\sim 10 \mu \mathrm{m}$ particle size $)$ was suspended in the solution, sonicated for $60 \mathrm{~s}$, followed by the solvent evaporation [355] or salt-leaching [356]. The mechanical properties obtained by this technique were about one-third that of trabecular bone. In a comparative study, PCL and biological apatite were mixed in the ratio 19:1 in an extruder [357]. At the end of the preparation, the mixture was cooled in an atmosphere of nitrogen. The authors observed that the presence of biological apatite improved the modulus while concurrently increasing the hydrophilicity of the polymeric substrate. Besides, an increase in apatite concentration was found to increase both the modulus and yield stress of the composite, which indicated to good interfacial interactions between the biological apatite and PCL. It was also observed that the presence of biological apatite stimulated osteoblasts attachment to the biomaterial and cell proliferation [357]. In another study, a PCL/HA biocomposite was prepared by blending in melt form at $120{ }^{\circ} \mathrm{C}$ until the torque reached equilibrium in the rheometer that was attached to the blender [358]. Then the sample was compression molded and cut into specimens of appropriate size for testing. It was observed that the composite containing $20 \mathrm{wt} \% \mathrm{HA}$ had the highest strength [358]. However, a direct grafting of PCL on the surface of HA particles seems to be the most interesting preparation technique [345]. In another study, HA porous scaffolds were coated by a PCL/HA composite coating [359]. In this system, PCL, as a coating component, was able to improve the brittleness and low strength of the HA scaffolds, while the particles in the coating were to improve the osteoconductivity 
and bioactivity of the coating layer. More complex formulations, such as PDLLA/PCL/HA [360], PLLA/PCL/HA [361], FA-HA/PCL [362], magnetic PCL/Fe-doped HA [363] and supramolecular PCL/functionalized HA [364,365] biocomposites, have been prepared as well. Further details on both the PCL/HA biocomposites and the processing methodologies thereof might be found elsewhere [262,349].

An interesting phenomenon of fractal growth of FA/gelatin composite crystals (Figure 3) was achieved by diffusion of calcium- and orthophosphate + fluoride-containing solutions from the opposite sides into a tube filled with a gelatin gel [366-372]. The reasons of this phenomenon are not quite clear yet. Other types of $\mathrm{CaPO}_{4}$-based composites, based on DCPD and OCP, were grown by the similar technique in an iota-carrageenan gel [373]. Up to now, nothing has yet been reported on a possible biomedical application of such unusual structural composites.

To finalize this section, one should note that there are polymers possessing shape-memory properties [374] and, therefore, $\mathrm{CaPO}_{4}$-based composites with such polymers appear to have the shape-memory properties as well [375-377].

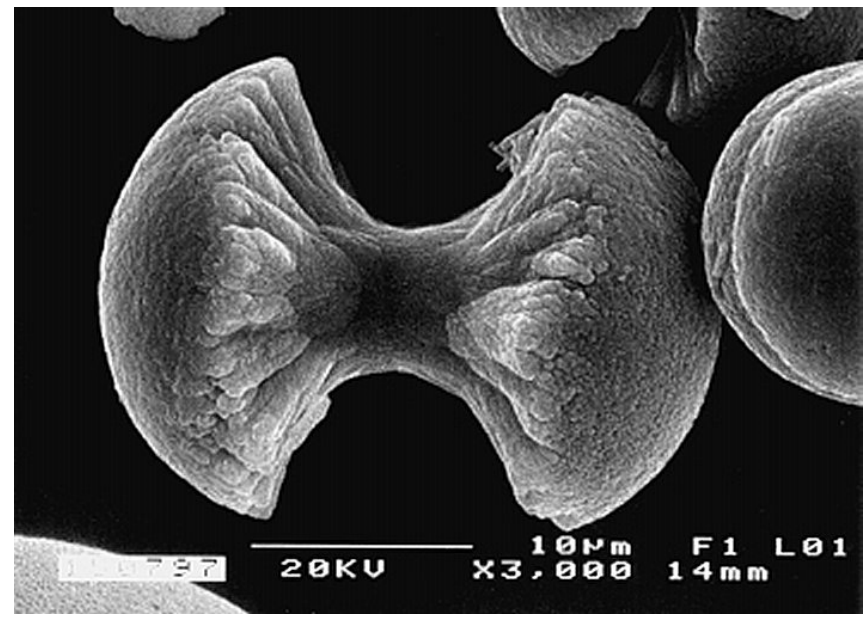

Figure 3. A biomimetically grown aggregate of fluorapatite (FA) that was crystallized in a gelatin matrix. Its shape can be explained and simulated by a fractal growth mechanism. Scale bar: $10 \mu \mathrm{m}$. Reprinted from Ref. [366] with permission.

\subsubsection{TCP-Based Formulations}

Both $\alpha$-TCP and $\beta$-TCP have a higher solubility than HA (Table 3). Besides, they are faster resorbed in vivo (however, there are some reports about a lack of TCP biodegradation after implantation in calvarial defects [378]). Therefore, $\alpha$-TCP and $\beta$-TCP were widely used instead of apatites to prepare completely biodegradable biocomposites [379-399]. For example, a biodegradable and osteoconductive biocomposite made of $\beta$-TCP particles and gelatin was proposed [383]. This material was tested in vivo with good results. It was found to be biocompatible, osteoconductive and biodegradable with no need for a second surgical operation to remove the device after healing occurred. Both herbal extracts [384] and $\mathrm{K}_{2} \mathrm{HPO}_{4}$ [385] might be added to this formulation. Another research group prepared biocomposites of crosslinked gelatin with $\beta$-TCP and both a good biocompatibility and bone formation upon subcutaneous implantation in rats were found [386]. Yang et al., [390] extended this to porous (porosity $\sim 75 \%$ ) $\beta$-TCP/gelatin biocomposites those also contained BMP-4. Furthermore, cell-compatible and possessive some osteoinductive properties porous $\beta$-TCP/alginate-gelatin hybrid scaffolds were prepared and 
successfully tested in vitro [387]. In addition, the $\mathrm{CaPO}_{4}$ fillers were found to have a reinforcing effect [400]. More to the point, biocomposites of $\beta$-TCP with PLLA [325,379-381] and PLGC [382] were prepared. Although $\beta$-TCP was able to counter the acidic degradation of the polyester to some extent, it did not prevent a $\mathrm{pH}$ drop down to $\sim 6$. Nevertheless, implantation of this biocomposite in beagles' mandibular bones was successful [382]. $\alpha$-TCP/gelatin formulations are known as well [393].

Based on a self-reinforcement concept, biocomposites of TCP with polylactides were prepared and studied using conventional mechanical testing [401]. Resorbable scaffolds were fabricated from such biocomposites [402]. Chitosan was also used as the matrix for the incorporation of $\beta$-TCP by a solid/liquid phase separation of the polymer solution and subsequent sublimation of the solvent. Due to complexation of the functional groups of chitosan with calcium ions of $\beta$-TCP, these biocomposites had high compressive modulus and strength [403]. PCL/ $\beta$-TCP biocomposites were developed in other studies [404-408] and their in vitro degradation behavior was systematically monitored by immersion in simulated body fluid at $37^{\circ} \mathrm{C}$ [406]. To extend this topic further, PCL/ $\beta$-TCP biocomposites might be loaded by drugs [407].

An in vitro study with primary rat calvarial osteoblasts showed an increased cellular activity in the BMP-loaded samples [390]. Other researchers investigated BMP-2-loaded porous $\beta$-TCP/gelatin biocomposites (porosity 95\%, average pore size 180-200 $\mu \mathrm{m}$ ) [409] and confirmed the precious study. A long-term implantation study of PDLLA/ $\alpha$-TCP composites in a loaded sheep implant model showed good results after 12 months but a strong osteolytic reaction after 24 months. This was ascribed to the almost complete dissolution of $\alpha$-TCP to this time and an adverse reaction of the remaining PDLLA [410].

More complex $\mathrm{CaPO}_{4}$-based formulations are known as well. For example, there is a biocomposite consisting of three interpenetrating networks: TCP, CDHA and PLGA [411]. Firstly, a porous TCP network was produced by coating a PU foam by hydrolysable $\alpha$-TCP slurry. Then, a CDHA network was derived from self-setting $\mathrm{CaPO}_{4}$ formulations filled in the porous TCP network. Finally, the remaining open pore network in the CDHA/ $\alpha$-TCP structures was infiltrated with PLGA. This biocomposite consists of three phases with different degradation behavior. It was postulated that bone would grow on the fastest degrading network of PLGA, while the remaining $\mathrm{CaPO}_{4}$ phases would remain intact thus maintaining their geometry and load bearing capability [411].

\subsubsection{Formulations Based on Other Types of $\mathrm{CaPO}_{4}$}

The number of research publications devoted to formulations based on other types of $\mathrm{CaPO}_{4}$ is substantially lesser than those devoted to apatites and TCP. Biphasic calcium phosphate (BCP), which is a solid composite of HA and $\beta$-TCP (however, similar formulations of HA and $\alpha$-TCP, as well as of $\alpha$-TCP and $\beta$-TCP are known, as well [412]) appears to be most popular among the remaining types of $\mathrm{CaPO}_{4}$. For example, collagen coated BCP ceramics was studied and the biocompatibility towards osteoblasts was found to increase upon coating with collagen [413]. Another research group created porous PDLLA/BCP scaffolds and coated them with a hydrophilic PEG/vancomycin composite for both drug delivery purposes and surface modification [414]. More to the point, both PLGA/BCP [415,416] and PLLA/BCP [417] biocomposites were fabricated and their cytotoxicity and fibroblast properties were found to be acceptable for natural bone tissue reparation, filling and augmentation $[418,419]$. 
Besides, PCL/BCP [420,421], PTMC/BCP [422] and gelatin/BCP [423,424] biocomposites are known as well.

A choice of DCPD-based biocomposites of DCPD, albumin and duplex DNA was prepared by water/oil/water interfacial reaction method [247]. Core-shell type DCPD/chitosan biocomposite fibers were prepared by a wet spinning method in another study [425]. The energy-dispersive X-ray spectroscopy analysis indicated that $\mathrm{Ca}$ and $\mathrm{P}$ atoms were mainly distributed on the outer layer of the composite fibers; however, a little amount of $\mathrm{P}$ atoms remained inside the fibers. This indicated that the composite fibers formed a unique core-shell structure with shell of $\mathrm{CaPO}_{4}$ and core of chitosan [425]. A similar formulation was prepared for further applications in self-setting biocomposites [426]. DCPA/BSA biocomposites were synthesized through the co-precipitation of BSA on the nanodimensional particles of DCPA performed in ethanol [427]. Nanodimensional DCPA was synthesized and incorporated into dental resins to form dental biocomposites [428-431]. Although, this is beyond the biomedical subject, it is interesting to mention that some DCPD/polymer composites could be used as proton conductors in battery devices [432,433]. Nothing has been reported on their biocompatibility but, perhaps, sometime the improved formulations will be used to fabricate biocompatible batteries for implantable electronic devices.

Various ACP-based biocomposites and hybrid formulations for dental applications have been developed [434,435]. Besides, several ACP-based formulations were investigated as potential biocomposites for bone grafting [344,436-438] and drug delivery [439,440]. Namely, ACP/PPF biocomposites were prepared by in situ precipitation [437], while PHB/carbonated ACP and PHBHV/carbonated ACP biocomposites appeared to be well suited as slowly biodegradable bone substitution material [344].

Finally, information on the biomedically relevant OCP-based formulations might be found in a topical review [441].

\subsection{Self-Setting Formulations}

Inorganic self-setting $\mathrm{CaPO}_{4}$ formulations (cements) hardening in the body were introduced in the early 1980-s. Since then, they have been broadly studied and many formulations have been proposed. These formulations set and harden due to various chemical interactions among $\mathrm{CaPO}_{4}$ that finally lead to formation of a monolithic body consisting of either CHDA or DCPD with possible admixtures of other phases. Unfortunately, having a ceramic nature, the self-setting $\mathrm{CaPO}_{4}$ formulations are brittle after hardening and the setting time is sometimes unsuitable for clinical procedures [442]. Therefore, to improve their properties, various attempts have been performed to transform them into biocomposites, e.g., by adding hydroxycarboxylic acids [443-445], gelatin [389,446-449], osteocalcin/collagen [450], alginate [451], chitin [452], chitosan [453], silk fibroin [454], silanized HPMC [455], bioactive glass [456], magnetic nanoparticles [457], etc. More to the point, various reinforcement additives of different shapes and nature are widely used to improve the mechanical properties of the self-setting formulations $[458,459]$. Even carbon nanotubes are used for this purpose [460,461]. Although the biomaterials community does not use this term, a substantial amount of the reinforced formulations might be defined as $\mathrm{CaPO}_{4}$-based concretes [442]. The idea behind the concretes is simple: if a strong filler is present in the matrix, it might stop crack propagation. 
Various apatite-containing formulations based on PMMA [462-470] and polyethylmethacrylate [471,472] have been already developed. Such biocomposites might be prepared by dispersion of apatite powder into a viscous fluid of the polymer [473] and used for drug delivery purposes [474]. When the mechanical properties of the concretes composed of PMMA matrix and HA particles of various sizes were tested, the tensile results showed that strength was independent on particle sizes. After immersion into Ringer's solution, the tensile strength was not altered whereas the fatigue properties were significantly reduced. The biocompatibility of PMMA/HA formulations was tested in vivo and enhanced osteogenic properties of the implants compared to single-phase PMMA were observed [463-466]. It was shown that not only the mechanical properties of PMMA were improved but the osteoblast response of PMMA was also enhanced with addition of HA [464]. Thereby, by adding of $\mathrm{CaPO}_{4}$, a non-biodegradable PMMA was made more bioactive and osteoconductive, yielding a well-processible biocomposite concrete. As a drawback, the PMMA/HA formulations possess a low flexural, compressive and tensile strength.

Biocomposites made from $\mathrm{CaPO}_{4}$ and various types of resins appeared to possess comparable mechanical and biological properties to typical PMMA cement, leading to potential uses for implant fixation [170,172,475-477]. To improve the mechanical properties of the self-setting $\mathrm{CaPO}_{4}$-based formulations and stabilize them at the implant site, various researchers have resorted to formulations that set in situ, primarily through crosslinking reactions of the polymeric matrix. For example, TTCP was reacted with PAA, forming a crosslinked CDHA/calcium polyacrylate biocomposite [478]. In aqueous solutions, TTCP hydrolyzes to CDHA [16] and the liberated calcium cations react with PAA, forming the crosslinked network [478]. Reed et al., synthesized a dicarboxy polyphosphazene that can be crosslinked by calcium cations and CDHA/polyphosphazene biocomposites with a compressive strength $\sim 10 \mathrm{MPa}$ and of $\sim 65 \%$ porosity were prepared as a result [479]. To mimic PMMA cements, PFF/ $\beta$-TCP biocomposites were prepared with addition of vinyl monomer to crosslink PPF. As a result, quick setting and degradable formulations with a low heat output and compressive strengths in the range of $1-12 \mathrm{MPa}$ were prepared by varying the molecular weight of PPF, as well as the contents of the monomer, $\beta$-TCP, initiator and $\mathrm{NaCl}$, as a porogen [480,481]. An acrylic formulation with Sr-containing $\mathrm{HA}$ as a filler [100], an injectable polydimethylsiloxane/HA formulation [482], biocomposites consisting of PLGA microspheres and self-setting $\mathrm{CaPO}_{4}$-based formulations [483,484], as well as hybrid formulations of chitosan oligosaccharide/gelatin/CaPO 4 [485] were prepared as well.

In order to improve the mechanical properties of self-setting formulations, numerous researchers blended various polymers to them. For example, gelatin might be added to self-setting formulations, primarily to stabilize the paste in aqueous solution before it develops adequate rigidity and, secondly, to improve the compressive strength $[389,446,486]$. Adding rod-like fillers to the self-setting formulations also caused an improvement in the mechanical properties [486]. For example, PAA and PVA were successfully used to improve the mechanical properties of a TTCP + DCPD formulation but, unfortunately, with an inevitable and unacceptable reduction of both workability and setting time [487,488]. Similar findings were reported in the presence of sodium alginate and sodium polyacrylate [489]. Other polymers, such as polyphosphazene might be used as well [490-492]. Other examples of polymer/CaPO 4 self-setting formulations might be found elsewhere [493,494]. Metallic wires might be used as reinforcements as well [495].

Porous $\mathrm{CaPO}_{4}$ scaffolds with interconnected macropores $(\sim 1 \mathrm{~mm})$, micropores $(\sim 5 \mu \mathrm{m})$ and of high porosity $(\sim 80 \%)$ were prepared by coating PU foams with a TTCP + DCPA formulation, followed by 
firing at $1200{ }^{\circ} \mathrm{C}$. In order to improve the mechanical properties of the scaffolds, the open micropores of the struts were then infiltrated by a PLGA solution to achieve an interpenetrating bioactive ceramic/biodegradable polymer composite structure. The PLGA filled struts were further coated with a 58S bioactive glass/PLGA composite coating. The obtained complex porous biocomposites could be used as tissue engineering scaffolds for low-load bearing applications [496]. A more complicated construction, in which the PLGA macroporous phase has been reinforced with a bioresorbable TTCP + DCPA formulation, followed by surface coating of the entire construct by a non-stoichiomentic CDHA layer, has been designed as well [497]. In one more study, $\alpha$-TCP and poly(ethylenephosphate) sodium salt-coated PLGA microparticles were mixed with castor oil and water to form an oil-in-water emulsion. The resulting emulsion spontaneously set in a humidified atmosphere at ambient temperature. The PLGA particles incorporated within the set formulations were hydrolytically degraded, which resulted in the formation of an interconnected microporosity [498].

A porosity level of $42 \%-80 \%$ was introduced into self-setting $\mathrm{CaPO}_{4} /$ chitosan biocomposites by addition of the water-soluble mannitol [499]. Chitosan significantly improved the mechanical strength of the entire biocomposite [500]. A similar approach was used by other researchers who studied the effect of the addition of PLGA microparticles [501-504] (which can also be loaded with drugs or growth factors [505-507]) to self-setting $\mathrm{CaPO}_{4}$ formulations. These biocomposites were implanted into cranial defects of rats and a content of $\sim 30 \mathrm{wt}$. \% of the microparticles was found to give the best results [501], while the addition of a growth factor to the biocomposites significantly increased bone contact at 2 weeks and enhanced new bone formation at 8 weeks [507]. The in vivo rabbit femur implant tests showed that self-setting $\mathrm{PLGA} \mathrm{CaPO}_{4}$ formulations exhibited outstanding biocompatibility and bioactivity, as well as a better osteoconduction and degradability than self-setting formulations consisted solely from $\mathrm{CaPO}_{4}[502]$.

To finalize this topic, one should mention that $\mathrm{CaPO}_{4}$ might be added into self-setting calcium sulfate (plaster of Paris) formulations to improve their osteoconductivity [508,509]. Similarly, calcium silicates could be added to the self-setting $\mathrm{CaPO}_{4}$ formulations [510-513].

\subsection{Formulations Based on Nanodimensional $\mathrm{CaPO}_{4}$ and Nanodimensional Biocomposites}

Nanodimensional and nanophasic materials are the materials that have particles or grain sizes less than $100 \mathrm{~nm}$, respectively. However, before proceeding any further, one should clearly differentiate between the nanodimensional composites and the composites containing nanodimensional particles. The former might be any type of composites but disintegrated to particles with dimensions $<100 \mathrm{~nm}$, while the latter consist of two or more materials, in which at least one of the materials is of a nanometer-scale. Nevertheless, both options combine the properties of both components, thus broadening their functionality beyond the pure materials.

Nanodimensional and nanophasic materials have different mechanical and optical properties if compared to the large grained materials of the same chemical composition. Namely, they possess the unique surface properties, such as an increased number of atoms, grain boundaries and defects at the surface, huge surface area and altered electronic structure, if compared to the conventional micron-sized materials. For example, nanodimensional HA (size $\sim 67 \mathrm{~nm}$ ) has a higher surface roughness of $17 \mathrm{~nm}$ if compared to $10 \mathrm{~nm}$ for the conventional submicron size HA $(\sim 180 \mathrm{~nm})$, while the contact angles (a 
quantitative measure of the wetting of a solid by a liquid) are significantly lower for nanodimensional HA (6.1) if compared to the conventional HA (11.51). Additionally, the diameter of individual pores in nanodimensional HA compacts is five-times smaller (pore diameter $\sim 6.6 \AA$ ) than that in the conventional grain-sized HA compacts (pore diameter within 19.8-31.0 A) [514]. Besides, nanodimensional $\mathrm{CaPO}_{4}$ promote osteoblast cells adhesion, differentiation and proliferation, osteointegration and deposition of calcium containing minerals on its surface better than microcrystalline ones; thus enhancing formation of a new bone tissue within a short period [515-517]. More to the point, nanodimensional HA was found to cause apoptosis of the leukemia P388 cells [518].

Natural bones and teeth are hierarchical biocomposites of biological origin based on nanodimensional compounds because they consists of nano-sized blade-like crystals of biological apatite grown in intimate contact with the organic matrix rich in bioorganic fibers and organized in complicated hierarchical structures. Given the fact that the major bioorganic phase of bones is collagen, i.e., a natural polymer (Table 1), it is obvious that biocomposites of nanodimensional $\mathrm{CaPO}_{4}$ with biodegradable polymers should be advantageous as bone grafting materials. In such biocomposites, the inorganic phase would be responsible for the mechanical strength (hardness) and bioactivity, while the polymeric phase would provide the elasticity. In addition, the solubility of $\mathrm{CaPO}_{4}$ depends on their crystallite size (smaller crystals have a higher solubility) and on their carbonate content (higher carbonate content increases the solubility) [519]. To the author's best knowledge, among $\mathrm{CaPO}_{4}$ listed in Table 3, before very recently only apatites (CDHA, HA and, perhaps, FA) have been available in nanodimensional state. However, recently, nano-sized DCPA [428-430] and nano-sized MCPM [520] have been synthesized and applied to prepare biocomposites with strong ionic release to combat tooth caries. Presumably, all $\mathrm{CaPO}_{4}$ from Table 3 might be manufactured in nanodimensional and/or nanocrystalline state; however not all of them have been prepared yet [517].

A number of investigations have been conducted recently to determine the mineralization, biocompatibility and mechanical properties of the biocomposites based on various (bio)polymers and nanodimensional $\mathrm{CaPO}_{4}$ (mainly, $\mathrm{HA}$ ). Unfortunately, in the majority of the already published papers it often remained unclear whether "nanodimensional HA", in fact, represented the nanodimensional stoichiometric HA or a nanodimensional non-stoichiometric CDHA; therefore, no differentiation is possible between them. These studies covered biocomposites with PGA [521], PLA [176,267,522-533] and its copolymers with PGA [534-536] and PCL [537], collagen [538-548], collagen + PLA [549-554], collagen + PVA [555], collagen + alginate [556,557], alginate [558], gelatin [559-564], PPF [565-567], PA [243,244,568-575], PVA [271,272,576-578], PVAP [276], poly(ethylene-co-acrylic) acid [579,580], chitosan [581-589] and its derivatives [590], konjac glucomannan + chitosan [591], chitosan + gelatin [592], xanthan [593], PHEMA + PCL [594], PCL [317,354,595-597], PU [598], cellulose [41,42,599-602], Ti [602-604], $\mathrm{ZrO}_{2}$ [605-607], glasses [608,609], metals and alloys [610,611] and many other biocompatible hybrid formulations [215,256,264,270,342,612-631]. Furthermore, each from the aforementioned formulations might be covered by a layer of nanodimensional $\mathrm{CaPO}_{4}$, as it was done by Zandi et al. [632], who coated a biocomposite of nano-sized rods HA with gelatin by nano-sized HA. Several nanodimensional biocomposites were found to be applicable as carriers for delivery of drugs and growth factors [609,633-635], as well as promising vectors with ultrahigh gene loading and transfection efficiency [636]. Data are available on the excellent biocompatibility of such biocomposites [545]. The dispersion state of nano-sized particles appears to be the critical parameter in controlling the mechanical 
properties of nanodimensional biocomposites, as nano-sized particles always tend to aggregate owing to their high surface energy [342]. A comparison was made of the mechanical properties between biocomposites of PA with the nano-sized and micron-sized HA. The results showed that the bending and tensile strengths of the biocomposite increased with increasing content of nanodimensional HA but decreased with increasing micron-sized HA content [243]. A SEM image of the mineralized collagen fibrils, demonstrating homogeneity of the nanodimensional biocomposite and the close interaction between the mineral phase and the reconstituted collagen fibrils is shown in Figure 4 [637].

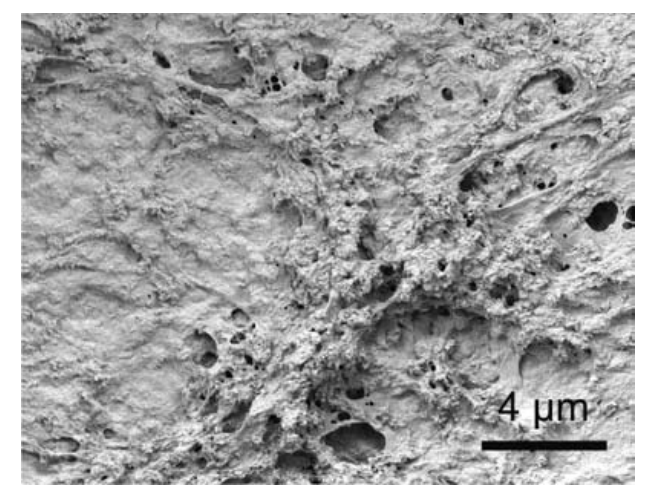

Figure 4. Scanning electron microscopy image of reconstituted mineralized collagen I fibrils. An example of an organic-inorganic nanostructural composite, mimicking the extracellular matrix of bone tissue on the nanometer scale. Reprinted from Ref. [637] with permission.

Porous (porosity $\sim 85 \%$ ) biocomposites of nano-sized HA with collagen and PLA have been prepared by precipitation and freeze-drying; these biocomposites did not show a $\mathrm{pH}$ drop upon in vitro degradation [549-551]. They were implanted in the radius of rabbits and showed a high biocompatibility and partial resorption after 12 weeks. Nano-sized HA/chitosan biocomposites with improved mechanical stability were prepared from HA/chitosan nano-sized rods [638]. Nano-sized HA/PLLA biocomposites of high porosity $(\sim 90 \%)$ were prepared using thermally induced phase separation [639]. Besides, nanodimensional HA was used to prepare biocomposites with PAA and the nanostructure of the resulting nano-sized crystals exhibited a core-shell configuration [640,641].

Nanodimensional crystals of HA appeared to be suitable for intra-osseous implantation and offered a potential to formulate enhanced biocomposites for clinical applications [642]. Thus, the biocompatibility of chitosan in osteoblast cell culture was significantly improved by addition of nano-sized HA [643]. Similar finding was valid for nanodimensional HA/PA biocomposites [569]. Further details on nanodimensional biocomposites might be found in excellent reviews [22,644]. More to the point, a more general review on applications of nanodimensional biomaterials in orthopedics is also available [645], where the interested readers are referred.

\subsection{Biocomposites with Collagen}

The main constituent of the bioorganic matrix of bones is type I collagen (Table 1) with molecules about $300 \mathrm{~nm}$ in length. The structural and biochemical properties of collagens have been widely investigated and over 25 collagen subtypes have been identified [646,647]. This protein is conducive to crystal formation in the associated inorganic matrix. It is easily degraded and resorbed by the body and 
allows good attachment to cells. Collagen alone is not effective as an osteoinductive material but it becomes osteoconductive in combination with $\mathrm{CaPO}_{4}$ [648]. Both collagen type I and HA were found to enhance osteoblast differentiation [649] but combined together, they were shown to accelerate osteogenesis. However, this tendency is not so straightforward: the data are available that implanted $\mathrm{HA} /$ collagen biocomposites enhanced regeneration of calvaria bone defects in young rats but postponed the regeneration of calvaria bone in aged rats [650]. Finally, addition of $\mathrm{CaPO}_{4}$ to collagen sheets was found to give a higher stability and an increased resistance to 3D swelling compared to the collagen reference [651]. Therefore, a bone analogue based on these two constituents should possess the remarkable properties.

The unique characteristics of bones are the spatial orientation between the nanodimentional crystals of biological apatite and collagen macromolecules at the nano-scale, where the crystals (about $50 \mathrm{~nm}$ length) are aligned parallel to the collagen fibrils, which is believed to be the source of the mechanical strength of bones (Figure 5a) [652]. The collagen molecules and the crystals of biological apatite assembled into mineralized fibrils are approximately $6 \mathrm{~nm}$ in diameter and $300 \mathrm{~nm}$ long. Although the complete mechanisms involved in the bone building strategy are still unclear, the strengthening effect of nanodimentional crystals of biological apatite in calcified tissues might be explained by the fact that the collagen matrix is a load transfer medium and thus transfers the load to the intrinsically rigid inorganic crystals [14,15,19-21]. Furthermore, the crystals of biological apatite located in between tangled fibrils cross-link the fibers either through a mechanical interlocking or by forming calcium ion bridges, thus increasing deformation resistance of the collagenous fiber network [653].

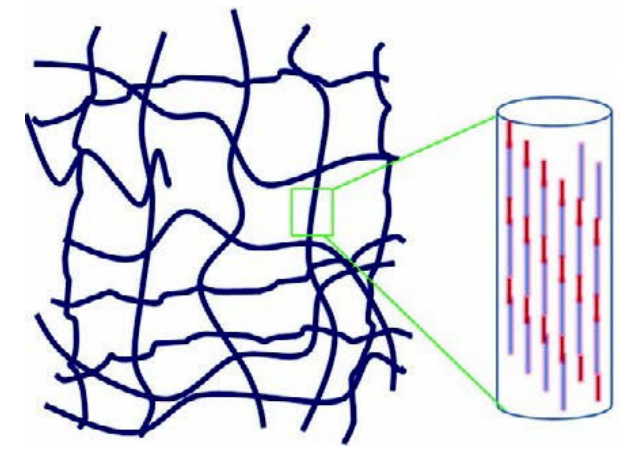

(a)

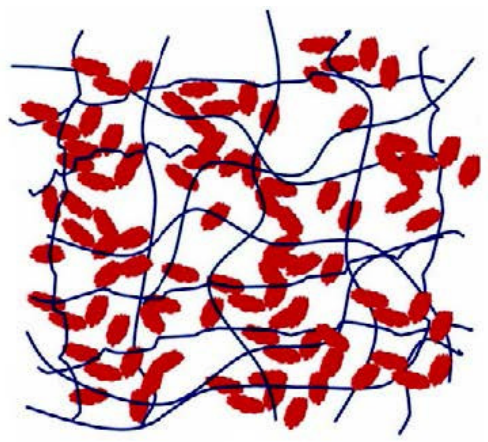

(b)

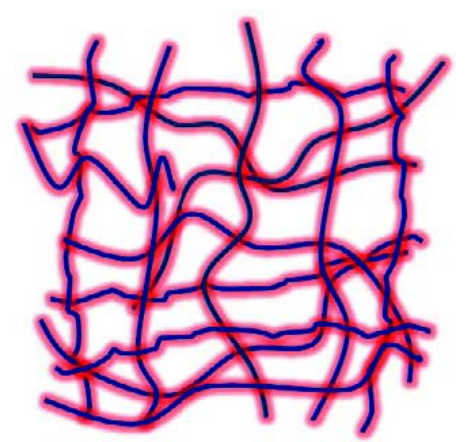

(c)

Figure 5. Schematic illustrations of collagen- $\mathrm{CaPO}_{4}$ nanocomposites: (a) structure of bone: nanodimensional crystals of biological apatite aligned along with the collagen fibrils; linking occurs between the apatite and polar groups on collagen chains in bone; (b) nanodimensional crystals of $\mathrm{CaPO}_{4}$ physically "trapped" within the collagen matrix; (c) nanodimensional crystals of $\mathrm{CaPO}_{4}$ deposited on and covered the collagen fibrils surface. Reprinted from Ref. [652] with permission.

When $\mathrm{CaPO}_{4}$ are combined with collagen in a laboratory, the prepared biocomposites appear to be substantially different from natural bone tissue (Figure $5 \mathrm{~b}, \mathrm{c}$ ) due to a lack of real interaction between the two components, i.e., the interactions that are able to modify the intrinsic characteristics of the singular components themselves. The main characteristics of the route, by which the mineralized hard tissues are formed in vivo, is that the organic matrix is laid down first and the inorganic reinforcing phase 
grows within this organic matrix $[14,15,20,21]$. Although to date, neither the elegance of the biomineral assembly mechanisms nor the intricate composite nano-sized architectures have been duplicated by nonbiological methods, the best way to mimic bone is to copy the way it is formed, namely by nucleation and growth of CDHA nano-sized crystals from a supersaturated solution both onto and within the collagen fibrils. Such syntheses were denoted as "biologically inspired"' which means they reproduce an ordered pattern and an environment very similar to natural ones [654-656]. The biologically inspired biocomposites of collagen and $\mathrm{CaPO}_{4}$ (mainly, apatites) for bone substitute have a long history [648,657-670] and started from the pioneering study by Banks et al., who grew CDHA on reconstituted calfskin collagen tapes in 1977 [671], followed by initial medical applications performed by other researchers in 1982 [672,673]. Such combinations were found to be bioactive, osteoconductive, osteoinductive $[648,674-676]$ and, in general, artificial grafts manufactured from this type of the biocomposites are likely to behave similarly to bones and be of more use in surgery than those prepared from any other materials. Indeed, data are available on the superiority of $\mathrm{CaPO}_{4} /$ collagen biocomposite scaffolds over the artificial polymeric and $\mathrm{CaPO}_{4}$ bioceramic scaffolds individually [677].

It has been found that $\mathrm{CaPO}_{4}$ may be successfully precipitated onto a collagen substrate of whatever form or source $[539,671,678,679]$. However, adherence of $\mathrm{CaPO}_{4}$ crystals to collagen did depend on how much the collagen had been denatured: the more fibrillar the collagen, the greater attachment. Clarke et al., first reported the production of a biocomposite produced by precipitation of DCPD onto a collagen matrix with the aid of phosphorylated amino acids commonly associated with fracture sites [657]. Self-setting CDHA forming formulations (DCPD + TTCP) have been mixed with a collagen suspension, hydrated and allowed to set. CDHA crystals were found to nucleate on the collagen fibril network, giving a material with the mechanical properties weaker than those reported for bone. More to the point, the prepared biocomposites were without the nanostructure similar to that of bone $[658,680]$. The oriented growth of OCP crystals on collagen was achieved by an experimental device in which $\mathrm{Ca}^{2+}$ and $\mathrm{PO}_{4}{ }^{3-}$ ions diffused into a collagen disc from the opposite directions [680-682]. Unfortunately, these experiments were designed to simulate the mechanism of in vivo precipitation of biological apatite only; due to this reason, the mechanical properties of the biocomposites were not tested [683].

Conventionally, collagen/CaPO 4 biocomposites can be prepared by blending or mixing of collagen and $\mathrm{CaPO}_{4}$, as well as by biomimetic methods [539,550,634,654-658,668,678,683-694]. Tampieri et al., [656] produced and compared artificial bone like tissue apatite/collagen biocomposites prepared by using two different methodologies: (1) dispersion of apatite in a collagen aqueous suspension and then freeze dried and (2) direct nucleation of an apatitic phase on assembling collagen fibrils. Biocomposites obtained using first way were similar to uncalcified natural collagen. The crystallite sizes were not uniform and were often aggregated and randomly distributed into the matrix (Figure 5b), proving that there was no real interaction between apatite and collagen fibers. However, the second method allowed the direct nucleation of nano-sized crystals of apatite on self-assembled collagen fibers. In this case, the two components (CDHA and collagen) exhibited strong interactions (Figure 5c), highlighted by several analysis techniques, which showed a complete analogy of the composite with calcified natural tissue [656]. Other production techniques are also possible. For example, using a polymer-induced liquid-precursor process, collagen/apatite biocomposites mimicking the nanostructure of bones, wherein nano-sized crystals of apatite were embedded within the collagen fibrils, were prepared [693]. More complicated formulations, such as a magnetite enriched HA/collagen [695] and HA/collagen/PVA [696] biocomposites, 
have been developed as well. Recent investigations revealed that $\mathrm{CaPO}_{4} /$ collagen formulations might be printed by means of 3D printers [697].

In addition, collagen might be incorporated into various self-setting $\mathrm{CaPO}_{4}$-based formulations [658,680,698-702]. Typically, a type I collagen sponge is presoaked in $\mathrm{PO}_{4}{ }^{3-}$-containing a highly basic aqueous solution and then is immersed into $\mathrm{Ca}^{2+}$-containing solution to allow mineral deposition. Also, collagen I fibers might be dissolved in acetic acid and then this solution is added to phosphoric acid, followed by a neutralization synthesis (performed at $25^{\circ} \mathrm{C}$ and solution $\mathrm{pH}$ within $9-10$ ) between an aqueous suspension of $\mathrm{Ca}(\mathrm{OH})_{2}$ and the $\mathrm{H}_{3} \mathrm{PO}_{4} /$ collagen solution $[654,655]$. To ensure the quality of the final product, it is necessary to control the $\mathrm{Ca} / \mathrm{P}$ ionic ratio in the reaction solution. One way to do this is to dissolve a commercial $\mathrm{CaPO}_{4}$ in an acid; another one is to add $\mathrm{Ca}^{2+}$ and $\mathrm{PO}_{4}{ }^{3-}$ ions in a certain ratio to the solution and after that induce the reaction [703]. Biomimetically, one can achieve an oriented growth of CDHA crystals onto dissolved collagen fibrils in aqueous solutions via a self-organization mechanism [688]. Besides, CDHA crystallization from aqueous solutions might be performed in the presence of a previously dispersed collagen [539]. More to the point, collagen might be first dispersed in an acidic solution, followed by addition of calcium and orthophosphate ions and then co-precipitation of collagen and CDHA might be induced by either increasing the solution $\mathrm{pH}$ or adding mixing agents. Although it resulted in biocomposites with poor mechanical properties, pressing of the apatite/collagen mixtures at $40{ }^{\circ} \mathrm{C}$ under $200 \mathrm{MPa}$ for several days is also known [704]. Attempts have been performed for a computer simulation of apatite/collagen composite formation process [705]. It is interesting to note, that such biocomposites were found to possess some piezoelectric properties [706].

As the majority of the collagen/HA biocomposites are conventionally processed by anchoring micronsized HA particles into collagen matrix, it makes quite difficult to obtain a uniform and homogeneous composite graft. Besides, such biocomposites have inadequate mechanical properties; over and above, the proper pore sizes have not been achieved either. Further, microcrystalline HA, which is in contrast to nanocrystalline bone apatite, might take a longer time to be remodeled into a new bone tissue upon the implantation. In addition, some of the biocomposites exhibited very poor mechanical properties, probably due to a lack of strong interfacial bonding between the constituents. Furthermore, in all blended composites, the crystallite sizes of $\mathrm{CaPO}_{4}$ were not uniform and the crystals were often aggregated and randomly distributed within a fibrous matrix of collagen. Therefore, no structural similarity to natural bone was obtained and only a compositional similarity to that of natural bone was achieved. The aforementioned data clearly demonstrate that the chemical composition similar to bone is insufficient for manufacturing the proper grafts; both the mechanical properties and mimetic of the bone nanostructure are necessary to function as bone in recipient sites. There is a chance for improving osteointegration by reducing the grain size of HA crystals by activating of ultrafine apatite growth into the matrix. This may lead to enhance the mechanical properties and osteointegration with improved biological and biochemical affinity to the host bone. Besides, the porosity was found to have a positive influence on the ingrowth of the surrounding tissues into the pores of collagen/HA biocomposites [707,708].

Bovine collagen might be mixed with $\mathrm{CaPO}_{4}$ and such biocomposites are marketed commercially as bone-graft substitutes those further can be combined with bone marrow aspirated from the iliac crest of the site of the fracture. Bioimplant ${ }^{\circledR}$, Bio-Oss Collagen ${ }^{\circledR}$, Boneject $^{\circledR}$, Collagraft $^{\circledR}$, CollapAn $^{\circledR}, \operatorname{Healos}^{\circledR}$, Integra Mozaik $^{\circledR}$, and $\mathrm{LitAr}^{\circledR}$ are several examples of the commercially available $\mathrm{CaPO}_{4} /$ collagen grafts for the clinical use [17,22]. Application of these materials was compared with autografts for the 
management of acute fractures of long bones with defects, which had been stabilized by internal or external fixation $[709,710]$. These biocomposites are osteogenic, osteoinductive and osteoconductive; however, they lack the structural strength and require a harvest of the patient's bone marrow. OCP/collagen biocomposites have been investigated [711] and clinically tested [712].

Collagen sponges with an open porosity $(30-100 \mu \mathrm{m})$ were prepared by a freeze-drying technique and then their surface was coated by a $10-\mu \mathrm{m}$ layer of biomimetic apatite precipitated from simulated body fluid [713]. The researchers found a good in vitro performance with fibroblast cell culture. Other preparation techniques are also possible [714]. Collagen/HA microspheres or gel beads have been prepared in the intention of making injectable bone fillers [715-717]. Liao et al. succeeded in mimicking the bone structure by blending carbonateapatite with collagen [718]. A similar material (mineralized collagen) was implanted into femur of rats and excellent clinical results were observed after 12 weeks [719]. Collagen/HA biocomposites were prepared and their mechanical performance was increased by crosslinking the collagen fibers with glutaraldehyde [540-542]. These biocomposites were tested in rabbits and showed a good biological performance, osteoconductivity and biodegradation. A similar approach was selected to prepare $\mathrm{HA} /$ collagen microspheres (diameter $\sim 5 \mu \mathrm{m}$ ) by a water-oil emulsion technique in which the surface was also cross-linked by glutaraldehyde [716]. That material showed a good in vitro performance with osteoblast cell culture. A porous bone graft substitute was formed from a nano-sized HA/collagen biocomposite combined with PLA by a freeze-drying method; the resulting material was found to mimic natural bones at several hierarchical levels [550]. Subsequent in vitro experiments confirmed a good adhesion, proliferation and migration of osteoblasts into this composite [549]. A further increase in biocompatibility might be achieved by addition of various dopants. For example, to enhance bone substitution, Si-substituted HA/collagen composites have been developed with silicon located preferentially in the collagen phase [541]. Porous (porosity level $\sim 95 \%$ with interconnected pores of $50-100 \mu \mathrm{m}$ ) biocomposites of collagen (crosslinked with glutaraldehyde) and $\beta$-TCP have been prepared by a freeze-drying technique, followed by sublimation of the solvent; the biocomposites showed a good biocompatibility upon implantation in the rabbit jaw [720].

Biocomposites of $\mathrm{CaPO}_{4}$ with collagen were found to be useful for delivery of drugs, growth factors and other important biomolecules [557,660,702,721-723]. Namely, an HA/collagen - alginate $(20 \mu \mathrm{L})$ with the rhBMP-2 $(100 \mu \mathrm{g} / \mathrm{mL}, 15 \mu \mathrm{L})$ showed bone formation throughout the implant 5 weeks after implantation without obvious deformation of the material [557]. Gotterbarm et al., developed a twolayered collagen/ $\beta$-TCP implant augmented with chondral inductive growth factors for repair of osteochondral defects in the trochlear groove of minipigs. This approach might be a new promising option for the treatment of deep osteochondral defects in joint surgery [722].

To conclude this part, one should note that biocomposites of apatites with collagen are a very hot topic for research and up to now, just a few papers have been devoted to biocomposites of other $\mathrm{CaPO}_{4}$ with collagen [685-687,722,724-726]. These biomaterials mimic natural bones to some extent, while their subsequent biological evaluation suggests that they are readily incorporated into the bone metabolism in a way similar to bone remodeling, instead of acting as permanent implant [550,673]. However, the performance of these biocomposites depends on the source of collagen from which it was processed. Several attempts have been made to simulate the collagen-HA interfacial behavior in real bone by means of crosslinking agents such as glutaraldehyde [540,542,543,678,716,720] with the purpose to improve the mechanical properties of these biocomposites. Unfortunately, a further progress 
in this direction is restricted by a high cost, difficulty to control cross-infection, a poor definition of commercial sources of collagens, as well as by a lack of an appropriate technology to fabricate bone-resembling microstructures. Further details on $\mathrm{CaPO}_{4} /$ collagen biocomposites might be found elsewhere [652,663,727].

\subsection{Formulations with Other Bioorganic Compounds}

The biggest practical problems with collagen type I are its cost and the poor definition of commercial sources of this material, which makes it difficult to follow up on well controlled processing. Therefore, collagen type I can be replaced by other compounds. One should notice, that, besides collagen, both human and mammalian bodies contain dozens types of various bioorganic compounds, proteins and biological macromolecules. Therefore, the substantial amount of them potentially might be used to prepare biocomposites with $\mathrm{CaPO}_{4}$. For example, a biologically strong adhesion (to prevent invasion of bacteria) between teeth and the surrounding epithelial tissues is attributed to a cell-adhesive protein, laminin [728]. In order to mimic the nature, a laminin/apatite biocomposite layer was successfully created on the surface of both titanium [729] and EVOH [730,731] using the biomimetic approach. A more complicated laminin/DNA/apatite biocomposite layer was found to be an efficient gene transfer system [732]. Further details on this subject are available in a topical review [733].

$\mathrm{CaPO}_{4} /$ gelatin biocomposites are widely investigated as potential bone replacement biomaterials [253,383-390,409,446-448,559-564,734-739]. For example, gelatin foams were successfully mechanically reinforced by HA and then crosslinked by a carbodiimide derivative [253]. Such foams were shown to be a good carrier for antibiotic tetracycline [735]. Several biocomposites of $\mathrm{CaPO}_{4}$ with alginates have been prepared [387,556,557,560,655,740-744]. For example, porous HA/alginate composites based on hydrogels were prepared both biomimetically [655] and by using a freeze-drying technique [740]. More complicated formulations have been developed as well [745].

Various biocomposites of $\mathrm{CaPO}_{4}$ with chitosan [234,403,425,436,453,462,499,581-591,618,638, 643,746-757] and chitin [392,452,758-762] are also very popular. For example, a solution-based method was developed to combine HA powders with chitin, in which the ceramic particles were uniformly dispersed [758,759]. Unfortunately, it was difficult to obtain the uniform dispersions. The mechanical properties of the final biocomposites were not very good; due to a poor adhesion between the filler and the matrix both the tensile strength and modulus were found to decrease with increase of the HA amount. Microscopic examination revealed that HA particles were intervened between the polymer chains, weakening their interactions and decreasing the entire strength [758,759]. Other manufacturing techniques might be found in the aforementioned references, for example, a HA/chitosan biocomposite was produced by a hydrothermal process from natural $\mathrm{CaCO}_{3} /$ chitosan biocomposite of crab shells [751]. Similarly, HA/chitosan biocomposite was produced by a hydrothermal process from previously prepared DCPD/chitosan biocomposite [752]. Biocomposites of natural HA with chitosan were found to possess both a good hard tissue biocompatibility and an excellent osteoconductivity, which is suitable for artificial bone implants and frame materials of tissue engineering [748]. Data are available that addition of $\mathrm{CaPO}_{4}$ into chitosan improved cell attachment, provided a higher cell proliferation and well-spread morphology when compared to chitosan alone [584,754]. More complex formulations, such as silk fibers reinforced HA/chitosan [763] and HA/collagen/chitosan [764] biocomposites, have been 
studied as well. Besides biomedical applications, biocomposites of nano-sized HA with chitin/chitosan might be used for removal of $\mathrm{Fe}$ (III) [765] and fluorides [766,767] from aqueous solutions.

Biocomposites of CDHA with water-soluble proteins, such as BSA, might be prepared by a precipitation method [493,768-771]. In such biocomposites, BSA is not strongly fixed to solid CDHA, which is useful for a sustained release. However, this is not the case if a water/oil/water interfacial reaction route has been used [247]. To extend this subject, inclusion of DNA into CDHA/BSA biocomposites was claimed [247,772-774]. Furthermore, biocomposites of an unspecified $\mathrm{CaPO}_{4}$ with DNA [775,776], as well as those of nano-sized crystals of biomimetic apatite with $\mathrm{C}_{60}$ fullerene and Au-DNA nano-sized particles [777] were prepared as well.

Akashi and co-workers developed a procedure to prepare $\mathrm{CaPO}_{4}$-based biocomposites by soaking hydrogels in supersaturated by $\mathrm{Ca}^{2+}$ and $\mathrm{PO}_{4}{ }^{3-}$ ions solutions in order to precipitate $\mathrm{CDHA}$ in the hydrogels (up to $70 \%$ by weight of CDHA could be added to these biocomposites) [778]. This procedure was applied to chitosan; the 3D shape of the resulting biocomposite was controlled by the shape of the starting chitosan hydrogel [779]. Another research group developed biocomposites based on in situ $\mathrm{CaPO}_{4}$ mineralization of self-assembled supramolecular hydrogels [780]. Other experimental approaches are also possible [781].

Various biocomposites of CDHA with glutamic and aspartic amino acids, as well as poly-glutamic and poly-aspartic amino acids have been prepared and investigated [277,278,782-785]. These (poly)amino acids were quantitatively incorporated into CDHA crystals, provoking a reduction of the coherent length of the crystalline domains and decreasing the crystal sizes. The relative amounts of the (poly)amino acid content in the solid phase, determined through HPLC analysis, increased with their concentration in solution up to a maximum of about $7.8 \mathrm{wt} \%$ for CDHA/aspartic acid and $4.3 \mathrm{wt} \%$ for CDHA/glutamic acid biocomposites. The small crystal dimensions, which implied a great surface area, and the presence of (poly)amino acids were suggested to be relevant for possible application of these biocomposites for hard tissues replacement [277,278,782-785]. A schematic description of a biocomposite formation from amino acids and ACP is shown in Figure 6 [786].

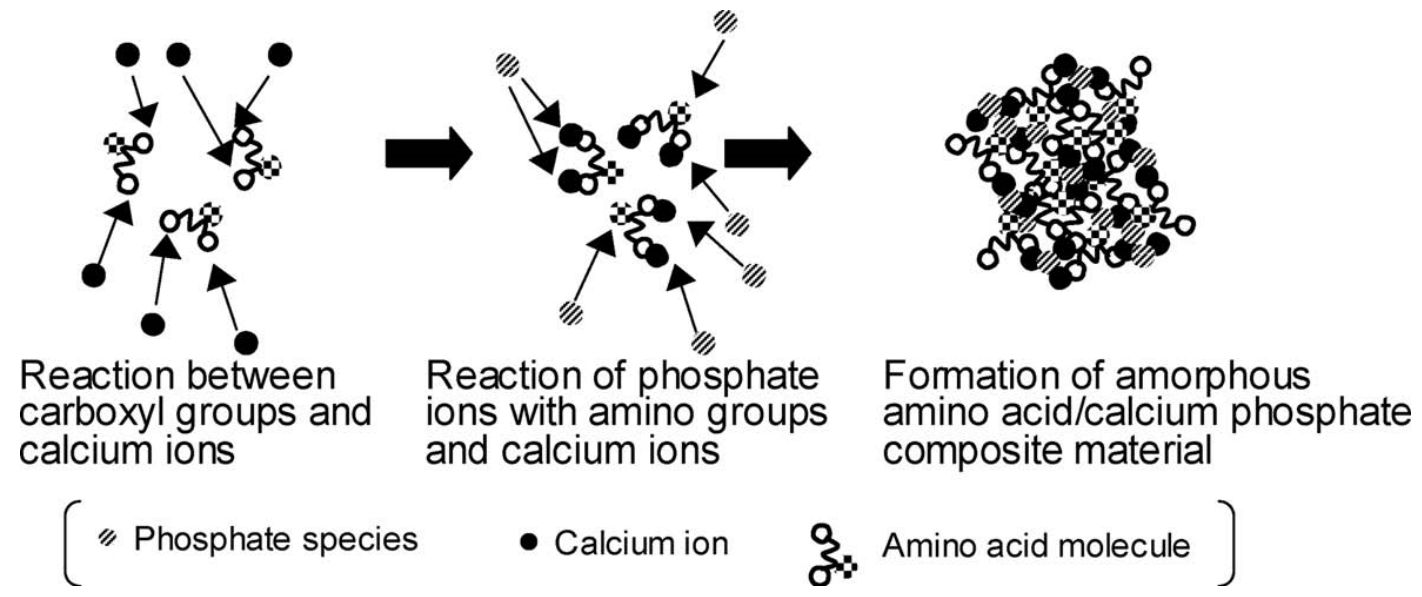

Figure 6. A proposed mechanism for the formation of amorphous calcium phosphates (ACP)/amino acid biocomposites in aqueous solutions. Reprinted from Ref. [786] with permission. 
Furthermore, BCP (HA $+\beta$-TCP)/agarose macroporous scaffolds with controlled and complete interconnection, high porosity, thoroughly open pores and tailored pore size were prepared for tissue engineering application [787,788]. Agarose, a biodegradable polymer, was selected as the organic matrix because it was a biocompatible hydrogel, which acted as gelling agent leading to strong gels and fast room temperature polymerization. Porous scaffolds with the designed architecture were manufactured by combining a low temperature shaping method with stereo-lithography and two drying techniques. The biocompatibility of this BCP/agarose system was tested with mouse L929 fibroblast and human SAOS-2 osteoblast during different colonization times [789].

Fibrin sealants are non-cytotoxic, fully resorbable, biological matrices that simulate the last stages of a natural coagulation cascade, forming a structured fibrin clot similar to a physiological clot [790]. Biocomposites of $\mathrm{CaPO}_{4}$ with fibrin sealants might develop the clinical applications of bone substitutes. The 3D mesh of fibrin sealant interpenetrates the macro- and micro-porous structure of $\mathrm{CaPO}_{4}$ ceramics. The physical, chemical and biological properties of $\mathrm{CaPO}_{4}$ bioceramics and the fibrin glue might be cumulated in biocomposites, suitable for preparation of advanced bone grafts [791-797].

Furthermore, there are biocomposites of $\mathrm{CaPO}_{4}$ with bisphosphonates [798], silk fibroin [245,619, 620,799-804], cellulose [805], chitosan + silk fibroin [806], chitosan derivatives [807], fibronectin [808], chondroitin sulfate [235,675,809], casein phosphopeptides [810], okra hydrocolloids [811], keratin hydrogel [812], amyloid fibrils [813], agarose [814] and vitamins [815]. Photopolymerisable formulations have been developed as well [816]. Besides, the reader's attention is pointed out to an interesting approach to crystallize CDHA inside poly(allylamine)/poly(styrene sulfonate) polyelectrolyte capsules resulting in empty biocomposite spheres of micron size. Depending on the amount of precipitated CDHA, the thickness of the shell of biocomposite spheres can be varied between 25 and $150 \mathrm{~nm}$. These biocomposite capsules might find application as medical agents for bone repairing and catalytic microreactors [817].

\subsection{Injectable Bone Substitutes (IBS)}

With the development of minimally invasive surgical methods, for example percutaneous surgery, directly injectable biomaterials are needed. The challenge is to place a biomaterial at the site of surgery by the least possible invasive method. In this regard, IBS appear to be a convenient alternative to solid bone-filling materials. They represent ready-to-use suspensions of $\mathrm{CaPO}_{4}$ microspheres [818,819], nanosized rods [820] or powder(s) in a liquid carrier phase. However, addition of other phases, such as calcium sulfate [821], is possible. IBS look like opaque viscous pastes with the rheological properties, sufficient to inject them into bone defects by means of surgical syringes and needles. Besides, IBS could be easily produced in a sterile stage. Their stable composition and mechanical properties are suitable for reproducibility of the biological response [822,823]. All types of IBS are divided into two major groups: self-setting formulations (see Section 4.2.) and those, which do not set. The latter ones are described here.

IBS requires suitable rheological properties to ensure bonding and cohesion of the mineral phase in situ with good cell permeability. Usually, the necessary level of viscosity is created by addition of suitable water-soluble polymers [92,251,564,824-832]. However, IBS based on hydrogels are known as well [833-835]. Therefore, the majority of $\mathrm{CaPO}_{4}$-containing IBS formulations might be considered as

a subgroup of $\mathrm{CaPO}_{4} /$ polymer biocomposites. For example, an IBS was described that involved a 
silanized hydroxyethylcellulose carrier with $\mathrm{BCP}(\mathrm{HA}+\beta-\mathrm{TCP})$. The suspension is liquid at $\mathrm{pH}$ within $10-12$, but gels quickly at $\mathrm{pH}<9$ [826]. Injectable composites can be formed with $\beta$-TCP to improve mechanical integrity [480]. Similarly, a polydioxanone-co-glycolide-based biocomposite reinforced with HA or $\beta$-TCP can be used as an injectable or moldable putty [827]. During the crosslinking reaction following injection, carbon dioxide is released allowing the formation of interconnected pores. Furthermore, HA/poly(L-lactide-co-e-caprolactone) biocomposite microparticles were fabricated as an injectable scaffold via the Pickering emulsion route in the absence of any molecular surfactants. A stable injectable oil-in-water emulsion was obtained using water dispersed HA nano-sized crystals as the particulate emulsifier and a dichloromethane solution of poly(L-lactide-co-e-caprolactone) as an oil phase [251]. $\mathrm{CaPO}_{4}$-containing IBS based on other (bio)organic hydrogels, such as gelatin [564], CMC [830], oligo(poly(ethylene glycol) fumarate) [828,829], chitosan + collagen [831] have been developed as well. In addition, photocrosslinkable formulations are known [832].

Daculsi et al. developed viscous IBS biocomposites based on BCP $(60 \% \mathrm{HA}+40 \% \beta-\mathrm{TCP})$ and $2 \%$ aqueous solution of HPMC that was said to be perfectly biocompatible, resorbable and easily fitted bone defects (due to an initial plasticity) [825,836-843]. The best ratio BCP/HPMC aqueous solution was found to be at $\sim 65 / 35 \mathrm{w} / \mathrm{w}$. To extend this subject further, IBS might be loaded by cells $[844,845]$, radiopaque elements [846] or microparticles [847], as well as functionalized by nucleic acids [848]. Selfhardening formulations, based on Si-HPMC hydrogel, are known as well [844]. The list of the commercially available $\mathrm{CaPO}_{4}$-based IBS formulations is presented in Table 5 [848].

Table 5. A list of some commercial non-setting $\mathrm{CaPO}_{4}$-based injectable bone substitutes (IBS) and pastes with indication of producer, product name, composition (when available) and form [848].

\begin{tabular}{|c|c|c|c|}
\hline Producer & Product name & Composition & Form \\
\hline \multirow[b]{2}{*}{ ApaTech (UK) } & Actifuse $^{\mathrm{TM}}$ & HA, polymer and aqueous solution & pre-mixed \\
\hline & $\begin{array}{l}\text { Actifuse }^{\mathrm{TM}} \text { Shape; } \\
\text { Actifuse }^{\mathrm{TM}} \text { ABX }\end{array}$ & Si-substituted $\mathrm{CaPO}_{4}$ and a polymer & pre-mixed \\
\hline Baxter (US) & TricOs T; TricOs & $\begin{array}{c}\text { BCP }(60 \% \text { HA, } 40 \% \beta-\mathrm{TCP}) \\
\text { granules and Tissucol (fibrin glue) }\end{array}$ & to be mixed \\
\hline $\begin{array}{l}\text { Berkeley Advanced } \\
\text { Biomaterials }\end{array}$ & Bi-Ostetic Putty & not disclosed & not disclosed \\
\hline BioForm (US) & $\begin{array}{c}\text { Calcium hydroxylapatite } \\
\text { implant }\end{array}$ & $\begin{array}{l}\text { HA powder embedded in a mixture of } \\
\text { glycerine, water and CMC }\end{array}$ & pre-mixed \\
\hline \multirow{3}{*}{ Biomatlante (FR) } & In'Oss $^{\mathrm{TM}}$ & $\begin{array}{c}\text { BCP granules (60\% HA, 40\% } \beta \text {-TCP; } \\
0.08-0.2 \mathrm{~mm}) \text { and } 2 \% \text { HPMC }\end{array}$ & pre-mixed \\
\hline & $\mathrm{MBCPGel}^{\circledR}$ & $\begin{array}{c}\text { BCP granules }(60 \% \mathrm{HA}, 40 \% \beta-\mathrm{TCP} \\
0.08-0.2 \mathrm{~mm}) \text { and } 2 \% \mathrm{HPMC}\end{array}$ & pre-mixed \\
\hline & Hydr'Os & $\begin{array}{c}\mathrm{BCP} \text { granules (60\% HA, } 40 \% \beta-\mathrm{TCP} \\
\text { micro- and nano-sized particles) and } \\
\text { saline solution }\end{array}$ & pre-mixed \\
\hline $\begin{array}{l}\text { Degradable solutions } \\
\qquad(\mathrm{CH})\end{array}$ & Easy graft ${ }^{\mathrm{TM}}$ & $\begin{array}{c}\beta \text {-TCP or BCP granules }(0.45-1.0 \mathrm{~mm}) \\
\text { coated with } 10 \mu \mathrm{m} \text { PLGA, } \\
\text { N-methyl-2-pyrrolydone }\end{array}$ & to be mixed \\
\hline
\end{tabular}


Table 5. Cont.

\begin{tabular}{|c|c|c|c|}
\hline Producer & Product name & Composition & Form \\
\hline Dentsply (US) & Pepgen P-15 ${ }^{\circledR}$ flow & $\begin{array}{l}\text { HA }(0.25-0.42 \mathrm{~mm}), \mathrm{P}-15 \text { peptide and } \\
\text { aqueous Na hyaluronate solution }\end{array}$ & to be mixed \\
\hline DePuy Spine (US) & Healos ${ }^{\circledR} \mathrm{Fx}$ & HA $(20 \%-30 \%)$ and collagen & to be mixed \\
\hline Fluidinova (P) & $\begin{array}{l}\text { nanoXIM TCP } \\
\text { nanoXIM HA }\end{array}$ & $\begin{array}{c}\beta-\mathrm{TCP}(5 \% \text { or } 15 \%) \text { and water } \\
\text { HA }(5 \%, 15 \%, 30 \% \text { or } 40 \%) \text { and water }\end{array}$ & $\begin{array}{l}\text { pre-mixed } \\
\text { pre-mixed }\end{array}$ \\
\hline $\begin{array}{l}\text { Integra LifeSciences } \\
\text { (US) }\end{array}$ & $\begin{array}{l}\text { Mozaik Osteoconductive } \\
\text { Scaffold }\end{array}$ & $\beta$-TCP $(80 \%)$ and type 1 collagen $(20 \%)$ & to be mixed \\
\hline Mathys Ltd $(\mathrm{CH})$ & Ceros $^{\circledR}$ Putty/cyclOS ${ }^{\circledR}$ Putty & $\begin{array}{c}\beta \text {-TCP granules }(0.125-0.71 \mathrm{~mm} ; 94 \%) \\
\text { and recombinant Na hyaluronate } \\
\text { powder }(6 \%)\end{array}$ & to be mixed \\
\hline Medtronic (US) & Mastergraft ${ }^{\circledR}$ & $\begin{array}{c}\mathrm{BCP}(85 \% \mathrm{HA}, 15 \% \beta-\mathrm{TCP}) \text { and } \\
\text { bovine collagen }\end{array}$ & to be mixed \\
\hline Merz Aesthetics (GER) & RADIESSE $^{\circledR}$ & HA particles suspended in a gel & pre-mixed \\
\hline Osartis / AAP (GER) & Ostim $^{\circledR}$ & Nanocrystalline HA (35\%) and water $(65 \%)$ & pre-mixed \\
\hline Smith \& Nephew (US) & JAXTCP & $\begin{array}{c}\beta \text {-TCP granules and an aqueous solution of } \\
1.75 \% \mathrm{CMC} \text { and } 10 \% \text { glycerol }\end{array}$ & to be mixed \\
\hline Stryker (US) & Calstrux ${ }^{\mathrm{TM}}$ & $\beta$-TCP granules and CMC & to be mixed \\
\hline Teknimed (FR) & Nanogel & HA $(100-200 \mathrm{~nm})(30 \%)$ and water $(70 \%)$ & pre-mixed \\
\hline Therics (US) & Therigraft ${ }^{\mathrm{TM}}$ Putty & $\beta$-TCP granules and polymer & pre-mixed \\
\hline Zimmer (US) & Collagraft & $\begin{array}{l}\mathrm{BCP} \text { granules }(65 \% \mathrm{HA}, 35 \% \beta-\mathrm{TCP} \\
0.5-1.0 \mathrm{~mm}) \text {, bovine collagen and bone } \\
\text { marrow aspirate }\end{array}$ & to be mixed \\
\hline
\end{tabular}

The advanced characteristics of IBS come from their good rheological properties and biocompatibility and the ease of tissue regeneration. Although the fabrication of IBS biocomposites in most cases improved the mechanical properties of the system and provided the material with resistance to fluids penetration, these achievements were limited by the amount of polymer that can be added to the paste. For instance, Mickiewicz et al. reported that after a critical concentration (that depended on the type and molecular weight of the polymer, but was always around 10\%), the polymer started forming a thick coating on the crystal clusters, preventing them from interlocking, originating plastic flow and, as a consequence, decreasing mechanical properties [493]. More to the point, Fujishiro et al. reported a decrease in mechanical properties with higher amounts of gel, which was attributed to formation of pores due to leaching of gelatin in solution [486]. Therefore, it seems that mechanical properties, although improved by the addition of polymers, are still a limitation for the application of $\mathrm{CaPO}_{4}$-based IBS formulations in load-bearing sites [125]. Further details on IBS are available elsewhere [822,823].

\subsection{Biocomposites with Inorganic Compounds, Carbon and Metals}

To overcome the problem of poor mechanical properties of $\mathrm{CaPO}_{4}$ bioceramics, suitable biocomposites of $\mathrm{CaPO}_{4}$ reinforced by various inorganic materials, glasses and metals have been developed as well. Such biocomposites are mainly prepared by the common ceramic processing techniques such as thermal treatment after kneading [849-851], powder slurry coating [852] and metal-sol mixing [853]. For 
example, HA was combined with Bioglass ${ }^{\circledR}$ (Novabone Products, Alachua, FL) $[854,855]$ and with other glasses [856] to form glass-ceramics biocomposites. Other reinforcement materials for $\mathrm{CaPO}_{4}$ could be differentiated by either shape of the fillers, such as particles [857,858], platelets [859,860], whiskers [520,861-863], fibers [864-868], or their chemical composition. Namely, zirconia and PSZ [849-852,861,869-881], alumina [857,860,882-887], titania [888-896] and their mixtures [897], other oxides and their mixtures [863,898-905], silica and/or glasses [906-915], wollastonite [916-920], mullite [921-924], various metals and alloys [495,610,611,866,925-943], calcium sulfate [944-949], calcium carbonate [950,951], silicon carbide [623,862], barium titanate [952], zeolite [953], boron nitride [954], zirconium nitride [955], carbon [956] and several other materials [957-959] were added to $\mathrm{CaPO}_{4}$ to improve reliability. In addition, $\mathrm{CaPO}_{4}$-based biocomposites possessing piezoelectric properties have been proposed [960]. Interestingly, $\mathrm{Fe}_{3} \mathrm{O}_{4} / \mathrm{HA}$ composites possess photocatalytic properties [902,903]. More complicated formulations, such as HA/aluminum oxide/carbon nanotubes [961], have been developed as well. Unfortunately, significant amounts of the reinforcing phases are needed to achieve the desired properties and, as these materials are either bioinert, significantly less bioactive than $\mathrm{CaPO}_{4}$ or not bioresorbable, the ability of the biocomposites to form a stable interface with bone is poorer if compared with $\mathrm{CaPO}_{4}$ bioceramics alone. Due to the presence of bioinert compounds, such formulations occasionally are called bioinert/bioactive composites [908]. The ideal reinforcement material would impart mechanical integrity to a biocomposite at low loadings, without diminishing its bioactivity.

There are several types of HA/glass biocomposites. The first one is also called bioactive glass-ceramics. A dense and homogeneous biocomposite was obtained after a heat treatment of the parent glass, which comprised $\sim 38$ wt \% oxy-FAP $\left(\mathrm{Ca}_{10}\left(\mathrm{PO}_{4}\right)_{6}(\mathrm{O}, \mathrm{F})_{2}\right)$ and $\sim 34 \mathrm{wt} \% \beta$-wollastonite $\left(\mathrm{CaO} \cdot \mathrm{SiO}_{2}\right)$ crystals, $50-100 \mathrm{~nm}$ in size in a $\mathrm{MgO}-\mathrm{CaO}-\mathrm{SiO}_{2}$ glassy matrix [916-920]. A-W glass-ceramics is an assembly of small apatite particles effectively reinforced by wollastonite. The bending strength, fracture toughness and Young's modulus of A-W glass-ceramics are the highest among bioactive glass and glass-ceramics, enabling it to be used in some major compression load bearing applications, such as vertebral prostheses and iliac crest replacement. It combines a high bioactivity with the suitable mechanical properties [962]. $\beta$-TCP/wollastonite biocomposites are also known [963-965]. More complicated formulations have been developed as well. For example, A-W/HDPE composite (AWPEX) biomaterials have been designed to match the mechanical strength of human cortical bone and to provide favorable bioactivity, with potential use in many orthopedic applications [966-969]. Similar can be said on A-W/silk fibroin biocomposites [970]. Other examples comprise wollastonite-reinforced HA/Ca polycarboxylate [971], glass-reinforced HA/polyacrylate [972], as well as collagen- [973] and gelatin- [974] calcium phosphate silicate/wollastonite biocomposites.

HA/glass biocomposites can be prepared by simple sintering of appropriate HA/glass powder mixtures [975-978]. If sintering is carried out below $1000^{\circ} \mathrm{C}$, HA does not react with the bioactive glass [976,977] or this reaction is limited [978]. Besides, reaction between HA and glasses depends on the glass composition. In another approach, small quantities of bioactive glass have been added to HA bioceramics in order to improve densification and/or mechanical properties [23]. In addition, biocomposites might be sintered from HA and silica [908]. In general, bioactive glass-ceramics maintain a high strength for a longer time than HA bioceramics under both the in vitro and in vivo conditions [910]. 
Due to a huge difference in shapes, it is a challenge to prepare homogeneous mixtures of $\mathrm{CaPO}_{4}$ and carbon nanotubes: "one can imagine something similar to achieving a homogeneous mixture of peas and spaghetti” [979] (p. 7). Nevertheless, different strategies might be employed to prepare $\mathrm{CaPO}_{4} / \mathrm{carbon}$ nanotube biocomposites. For example, apatites might be chemically synthesized by using carboxyl functionalized carbon nanotubes as a matrix [238-241,980-984]. Physico-chemical characterization of these biocomposites showed that nucleation of CDHA initiates through the carboxyl group [238]. Hot pressing [985], compaction [986], plasma spraying [987], laser surface alloying [988-990], spark plasma sintering [991], shear mixing [992], sol-gel [993] and precipitation [994] techniques might be applied as well. Due to carbon oxidation at elevated temperature, sintering of $\mathrm{CaPO}_{4} /$ carbon nanotube biocomposites must be performed in a deoxidizing atmosphere [995]. The research on $\mathrm{CaPO}_{4}$ (up to now, only apatite)/carbon nanotube biocomposites is in its early stages, with the first papers published in 2004 [980]. Due to this reason, the mechanical property data for such biocomposites have been reported only in few papers; however, these results are encouraging. For example, Chen et al., performed nano-indentation tests on biocomposite coatings to give hardness and Young's modulus values [990]. They found that the higher the loading of the nanotubes, the better the mechanical properties. Namely, at $20 \mathrm{wt} \%$ loading, hardness was increased by $\sim 43 \%$ and Young's modulus by $\sim 21 \%$ over a singlephase HA coating [990]. Scratching test results indicated that as alloyed HA biocomposite coatings exhibited improved wear resistance and lower friction coefficient with increasing the amount of carbon nanotubes in the precursor material powders [989]. Additionally, measurements of the elastic modulus and hardness of the biocomposite coatings indicated that the mechanical properties were also affected by the amount of carbon nanotubes [988]. Another research group performed compression tests on bulk HA/carbon nanotubes biocomposites and found an increase in strength over single-phase HA [980]. However, the highest compressive strength they achieved for any material was only $102 \mathrm{MPa}$, which is similar to that of cortical bone but much lower than the typical values for dense HA [979]. More complex formulations, such as poly-L-lysine/HA/carbon nanotube hybrid biocomposites, have been also developed [996]. Furthermore, $\mathrm{CaPO}_{4} /$ carbon nanotube biocomposites might be immobilized by hemoglobin [997]. Unfortunately, carbon nanotubes are very stable substances; they are neither bioresorbable nor biodegradable. Therefore, during in vivo bioresorption, the nanotubes will get into the human body from the biocomposite matrix and might cause uncertain health problems. Certainly, this problem must be solved. To conclude the carbon subject, one should mention on application of carbon fibers of microscopic dimensions [998-1000], nanodimensional diamonds [1001], graphene [1002-1007], fullerenes [777] and its derivatives [1008] to reinforce $\mathrm{CaPO}_{4}$ bioceramics.

As seen from the amount of the references, $\mathrm{CaPO}_{4} /$ zirconia biocomposites appear to be a hot topic of the research. The main disadvantage of HA reinforced by PSZ is degradation of zirconia in wet environments $[861,870,871,874]$. Transformation of the tetragonal $\mathrm{ZrO}_{2}$ to the monoclinic phase on the surface results in formation of microcracks and consequently lowers the strength of the implant $[1009,1010]$.

Various biocomposites of $\mathrm{CaPO}_{4}$ with metals and alloys have been fabricated as well [495,610,611,866,925-943]. For example, an HA-based biocomposite reinforced with 20 vol.\% of Ti particles was fabricated by hot pressing [927]. Besides, biocomposites of $\mathrm{CaPO}_{4}$ with metals might be prepared by powder metallurgy processing $[928,930,941,942]$ and mechanical compaction followed by sintering [611,936,939]. Furthermore, silver nanoparticles/HA biocomposites could be synthesized through $\gamma$-irradiation reduction of silver ions incorporated into HA particles [610]. At high temperatures, 
the presence of Ti metal phase was found to promote dehydration and decomposition of HA into $\beta$-TCP and TTCP $[927,928,939,1011]$ and/or partial formation of $\beta$-TCP and calcium titanates instead of HA [603,930,942,1011]. Comparing with pure HA bioceramics manufactured under the same conditions, the HA/Ti biocomposites possessed a higher fracture toughness, bending strength, work of fracture, which is better suitable for the biomedical applications [936]. However, the mechanical properties appeared to be not high enough to use HA/Ti biocomposites in load-bearing applications. Luckily, the histological evaluations revealed that HA/Ti biocomposites could be partially integrated with newborn bone tissues after 3 weeks and fully osteointegrated at 12 weeks in vivo [927]. Similar findings had been earlier made for HA bioceramics reinforced by addition of silver particulates (5-30 vol.\%) and subsequent sintering of the HA/Ag powder compacts $[925,926]$. Besides, addition of silver imparts an antimicrobial activity [932]. Other studies on $\mathrm{CaPO}_{4} / \mathrm{Ti}$ biocomposites are available elsewhere [929,931,942]. In all the aforementioned studies, $\mathrm{CaPO}_{4}$ was used as a matrix; however, the opposite situation is also possible. There is a study, in which composites made of $\mathrm{Mg} 3 \mathrm{Zn} 0.8 \mathrm{Zr}$ alloy as a matrix and $\beta$-TCP particles as reinforcements were prepared for investigating the effect of $\beta$-TCP spherical particles sized in $100 \mathrm{~nm}$ on the microstructure of that alloy [1012].

To conclude this part, biocomposites consisting of $\mathrm{CaPO}_{4}$ only should be briefly described. First, all types of biphasic, triphasic and multiphasic $\mathrm{CaPO}_{4}$ formulations should be mentioned [412]. For example, in 1980-s, BCP was called as "TCP ceramics complexed with HA" [1013]. Even nowadays BCP is occasionally called as a "nanocomposite" [1014]. Furthermore, fluoridated HA (described by a chemical formula $\mathrm{Ca}_{10}\left(\mathrm{PO}_{4}\right)_{6}(\mathrm{OH})_{2-x} \mathrm{~F}_{x}$, where $0<x<2$ ) might be mentioned as composites [1015]; however, an applicability of the "composite" term for such systems is doubtful. One should better consider 70\% HA-powder $+30 \%$ HA-whisker biocomposites, which were fabricated by pressureless sintering, hot pressing and hot isostatic pressing. These biocomposites were found to exhibit an improved toughness, attaining the lower fracture-toughness limit of bone without a decrease of bioactivity and biocompatibility [1016,1017]. Besides, a dual HA biocomposite that combined two HA materials with different porosities: HA with $75 \%$ porosity, for bone ingrowth and HA with $0 \%$ porosity, for load bearing was manufactured. This dual HA biocomposite appeared to be suitable for use as an implant material for spinal interbody fusion as a substitute for iliac bone grafts, which could eliminate the disadvantages associated with autograft harvesting [1018]. A biodegradable biocomposite porous scaffold comprising a $\beta$-TCP matrix and nano-sized fibers of HA was developed and studied for load-bearing bone tissue engineering. The nano-sized fibers of HA were prepared by a biomimetic precipitation method, the inclusion of which significantly enhanced the mechanical property of the scaffold, attaining a compressive strength of 9.87 MPa, comparable to the high-end value (2-10 MPa) of cancellous bone [1019]. Furthermore, HA/ $\beta$-TCP biocomposites with different $\beta$-TCP content $(10,20$, and $30 \mathrm{wt} \%)$ were prepared by wet mixing of HA and $\beta$-TCP powders, compaction of the powder mixtures and sintering [1020]. Finally, it is interesting to mention on a successful reinforcement of carbonateapatite porous blocks by newly prepared carbonateapatite crystals (i.e., by the same compound; thus, a biocomposite of two different carbonateapatites was obtained) [1021]. First, a calcium salt was introduced to micropores of carbonateapatite blocks. Then, the calcium salt was carbonated to form calcite inside the micropores of the carbonateapatite blocks by exposing the blocks to carbon dioxide at the second step. On the third step, the blocks were immersed in a $\mathrm{Na}_{2} \mathrm{HPO}_{4}$ aqueous solution. In this process, calcite inside the micropores of the carbonateapatite blocks was transformed to carbonateapatite and the newly formed 
crystals of carbonateapatite entangled on those of the existing carbonateapatite blocks. Due to bonding between the newly formed carbonateapatite crystals and the existing ones in the carbonateapatite blocks, a mechanical strength of the blocks became $\sim 1.5$ times higher when compared to that before the treatment [1021].

\subsection{Functionally Graded Formulations}

Although, in most cases, the homogeneous distribution of filler(s) inside a matrix is required [347], there are composites, in which this is not the case. For example, functionally graded materials (FGM) might be characterized by the intentional variations in composition and/or structure gradually over volume, resulting in corresponding changes in the properties of the composite. The main feature of such materials is the almost continuously graded composition that results in two different properties at the two ends of the structure. Such composites can be designed for specific function and applications. Various approaches based on the bulk (particulate processing), pre-form processing, layer processing and melt processing are used to fabricate the functionally graded materials.

Bone is a biologically formed composite with variable density ranging from very dense and stiff (cortical bone) to a soft and foamed structure (trabecular bone). Normally the outer part of long bones consists of cortical bone with the density decreasing towards the core, where the trabecular bone is found. The trabecular bone is porous and the porosity is filled with osseous medulla [14,15]. This brief description clearly indicates that bones are natural functionally graded composites.

The concept of FGM has been increasingly used for biomaterial design and currently it remains to be an important area of the research. For example, many studies have been performed to fabricate porositygraded $\mathrm{CaPO}_{4}$ bioceramics in attempts to mimic the porous structure of bones [1022-1025]. This is a structural approach to fabricate FGM. Besides, there is a compositional approach. For example, functionally graded composite skull implants consisting of polylactides, carbonateapatite and $\mathrm{CaCO}_{3}$ have been prepared [312,313]. In addition, powder metallurgy methods have been used to fabricate $\mathrm{HA} / \mathrm{Ti}$ functionally graded biocomposite dental implants offering the biocompatible HA on the tissue side and titanium on the outer side for mechanical strength [1026-1028]. The graded structure in the longitudinal direction contains more $\mathrm{Ti}$ in the upper section and more HA in the lower section. Actually, in the upper section the occlusal force is directly applied and $\mathrm{Ti}$ offers the required mechanical performance; in the lower part, which is implanted inside the bone, the HA confers the bioactive and osteoconductive properties to the material [1026]. Since the optimum conditions of sintering for Ti and $\mathrm{HA}$ are very different, HA/Ti functionally graded biocomposites are difficult to fabricate and the sintering conditions for their mixtures are obliged to compromise. The expected properties of this implant are shown in Figure 7 [1027]. Such biocomposites might be both symmetrical [1029] and asymmetrical [1030]. 

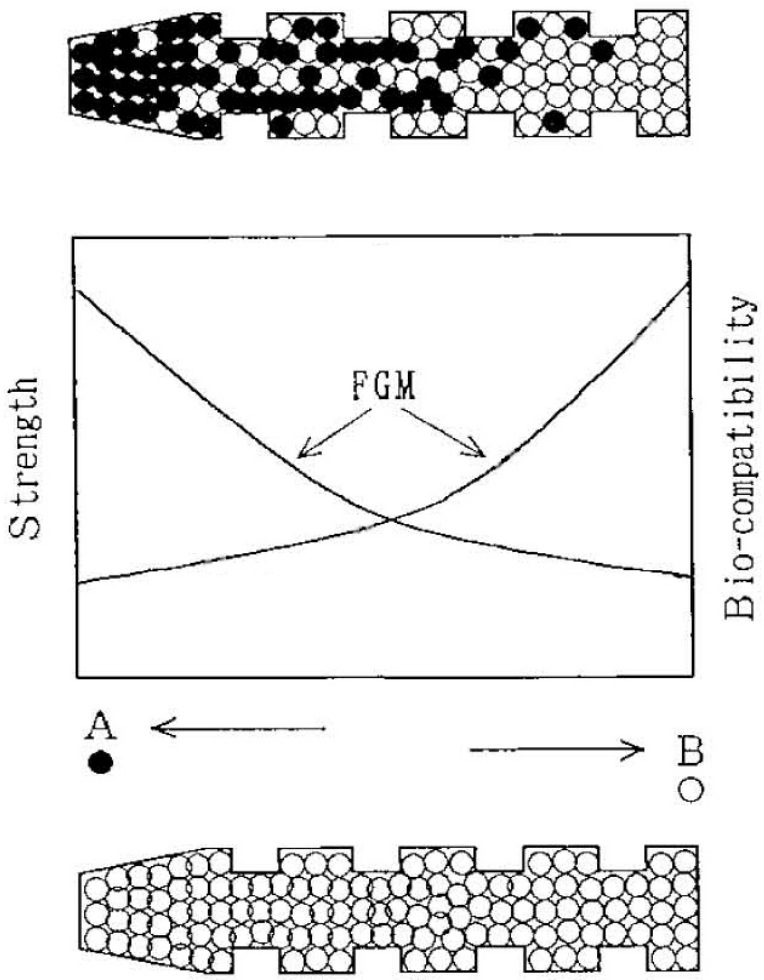

Figure 7. Expected properties of functionally graded biocomposite dental implant. For comparison, the upper drawing shows a functionally graded implant and the lower one shows a conventional uniform implant. The properties are shown in the middle. The implant with the composition changed from a biocompatible metal (Ti) at one end (left in the figure), increasing the concentration of bioceramics (hydroxyapatite (HA)) toward 100\% HA at the other end (right in the figure), could control both mechanical properties and biocompatibility without an abrupt change due to the formation of discrete boundary. This functionally gradient material (FGM) biocomposite was designed to provide more titanium for the upper part where occlusal force is directly applied and more HA for the lower part, which is implanted inside the jawbone. Reprinted from Ref. [1027] with permission.

A series of functionally graded $\mathrm{CaPO}_{4}$ coatings incorporated with various percentages of silver were deposited on titanium substrates using ion beam-assisted deposition. The analysis of the coating's crosssection revealed a decreased crystallinity as well as a distribution of nano-sized $(10-50 \mathrm{~nm})$ silver particles from the coating/substrate interface to top surface [1031]. Similarly, compositionally graded HA/Ti biocomposite coatings were prepared by rf-plasma spraying [1032]. In addition, both laser cladding [1033] and combinatorial matrix-assisted pulsed laser evaporation [1034] techniques were used to put down gradient deposits of carbon nanotubes/HA and Sr-substituted HA and zoledronate-modified $\mathrm{HA}$, respectively. A functionally graded HA/PMMA biocomposite was developed based on sedimentary HA distributions in a PMMA viscous fluid, using a centrifuge to avoid stress convergence on the interface. The stress-strain curves of this biocomposite showed a sufficient strength for biomedical applications along with the relaxation of brittleness and fragility [473]. A compositionally graded collagen/nanodimensional HA biocomposite scaffold might be prepared by an in situ diffusion method. Chemical and microstructural analysis revealed a gradient of the $\mathrm{Ca}$ to $\mathrm{P}$ ratio across the width of the scaffold template, resulting in the formation of a Ca-rich side and a Ca-depleted side of scaffold. The 
Ca-rich side featured low porosity and agglomerates of the nanodimensional HA crystallites, while the Ca-depleted side featured higher porosity and nanodimensional HA crystallites integrated with collagen fibrils to form a porous network structure [1035]. A three-layered graded biocomposite membrane, with one face of $8 \%$ nanodimensional carbonateapatite/collagen/PLGA porous membrane, the opposite face of pure PLGA non-porous membrane and the middle layer of $4 \%$ nanodimensional carbonateapatite/collagen/PLGA as the transition was prepared through the layer-by-layer casting method [552]. A similar PLGA/nano-HA/lauric acid graded biocomposite was prepared as well [1036]. Functionally graded non-woven meshes of PCL incorporated by nano-sized particles of $\beta$-TCP were prepared using a hybrid twin-screw extrusion/electrospinning process [1037]. A functionally graded HA/silk fibroin biocomposite was prepared by pulse electric current sintering [1038]. HA/glass FGM layers were coated on titanium alloy (Ti6A14V) substrates. The design of these layers and the use of the glass were for achieving a strong bonding between the FGM layered coatings and the substrates [1039,1040].

Functionally graded $\beta$-TCP/FA biocomposites combine the biostability of FA with the bioresorbable properties of $\beta$-TCP. An interesting multilayered (each layer of $1 \mathrm{~mm}$ thick) structure consisting of $\beta$ TCP/FA biocomposites with different molar ratios has been prepared, giving rise to formation of a FGM (Figure 8). After implantation, the preferential dissolution of $\beta$-TCP phase would result in functionally gradient porosity for bone ingrowth [1041]. In addition, compositionally graded HA-alumina-zirconia biocomposites were prepared by the same multilayered technique [1042]. Functionally graded fluoridated HA with a gradient of fluoride [1043] and carbonated HA with a gradient of carbonate [1044] were synthesized as well. HA/zirconia graded biocomposites were fabricated to enhance the mechanical properties of HA while retaining its bone bonding property [879]. $\mathrm{TiO}_{2}$ and $\mathrm{HA}$ were found to be a good combination for FGM providing both a gradient of bioactivity and a good mechanical strength [1045]. Besides, graded $\mathrm{HA} / \mathrm{CaCO}_{3}$ biocomposite structures for bone ingrowth were developed [1046]. Functionally graded composite skull implants consisting of polylactides, carbonateapatite and $\mathrm{CaCO}_{3}$ are known as well [312,313]. Thus, the research in this field is quite promising but currently the mechanical properties of the available biocomposites do not match the similar properties of bones [1047].

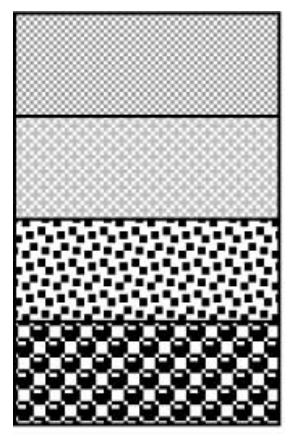

(a)

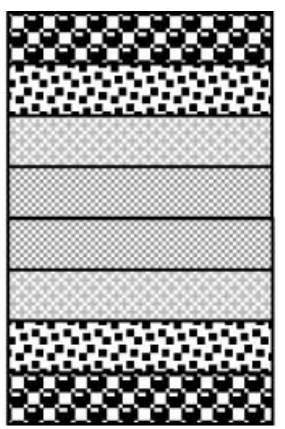

B8 $\mathrm{FA}+50 \mathrm{TCP}$

Fت FA + 40TCP

$\mathrm{FA}+30 \mathrm{TCP}$

$\mathrm{FA}+20 \mathrm{TCP}$

Figure 8. A schematic diagram showing the arrangement of the FA/ $\beta$-TCP biocomposite layers. (a) A non-symmetric functionally gradient material (FGM); (b) symmetric FGM. Reprinted from Ref. [1041] with permission. 


\subsection{Biosensors}

A biosensor is a device for detection of an analyte that combines a biological component with a physicochemical detector component. Very briefly, it consists of three parts: a sensitive biological element; a transducer or a detector element that transforms the signal resulting from the interaction of the analyte with the biological element into another signal; and associated electronics that is primarily responsible for the display of the results in a user-friendly way [1048].

The surface of biologically relevant $\mathrm{CaPO}_{4}$ (CDHA, HA, $\alpha$-TCP, $\beta$-TCP, DCPD, DCPA) has an excellent ability of adsorption for functional biomolecules such as proteins, albumins, DNA, as well as some other types of chemicals. Therefore, several $\mathrm{CaPO}_{4}$-based biocomposites and hybrid biomaterials were found to be applicable for manufacturing of biosensors [996,1049-1056]. For example, formation of poly-L-lysine/HA/carbon nanotube hybrid nanodimensional particles was described and a general design strategy for an immunosensing platform was proposed based on adsorption of antibodies onto this biocomposite [996]. In another paper, a hybrid material formed by assembling of nanodimensional particles of gold onto nano-sized HA was employed for the interface design of piezoelectric immunosensor, on which the antibodies were bound. The developed sensing interface appeared to possess some advantages, such as activation-free immobilization and high antigen-binding activities of antibodies, over using nano-sized either HA or gold alone [1050]. A novel tyrosinase biosensor based on nano-sized $\mathrm{HA} /$ chitosan composite has been developed for the detection of phenolic compounds [1054]. Further details on the subject are available in the aforementioned references.

Up to date, not many papers have been published on the biosensor application of $\mathrm{CaPO}_{4}$-based biocomposites and hybrid biomaterials. Presumably, this subject will be further developed in the future and, perhaps, sometime implantable biosensors will be designed to perform the continuous concentration monitoring of the important biological macromolecules in vivo. Possibly, those implantable biosensors will be able to use an electric power, generated by DCPD/polymer composite-based battery devices [432,433].

\section{Interactions among the Phases}

An important aspect that should be addressed in details is a mutual interaction between $\mathrm{CaPO}_{4}$ and other phases in biocomposites and hybrid biomaterials. In general, an interaction among the phases in any composite can be either mechanical, when it results from radial compression forces exerted by the matrix on the filler particles (for example, developed during cooling due to thermal contraction), or chemical, when the reactivity of the filler towards the matrix has an important role. In the latter case, it is important to distinguish a physical interaction from chemical bonding [219]. According to Wypych [1057], physical interaction is more or less temporary, implicating hydrogen bonding or van der Waals forces, whereas chemical bonding is stronger and more permanent, involving covalent bond formation. Thus, a chemical interfacial bond among the phases is preferred to achieve a higher strength of a composite. The magnitude of the interfacial bond among the phases determines how well a weak matrix transmits stress to the strong fibers. However, while a bond among the matrix and reinforcements must exist for the purpose of stress transfer, it should not be so strong that it prevents toughening mechanisms, such as de-bonding and fiber pullout [979]. 
There is still doubt as to the exact bonding mechanism among bone minerals (biological apatite) and bioorganics (collagen), which undoubtedly plays a critical role in determining the mechanical properties of bones. Namely, bone minerals are not directly bonded to collagen but through non-collagenous proteins that make up $\sim 3 \%$ of bones (Table 1) and provide with active sites for biomineralization and for cellular attachment [22]. In bones, the interfacial bonding forces are mainly ionic bonds, hydrogen bonds and hydrophobic interactions, which give the bones the unique composite behavior [322]. There is an opinion that, opposite to bones, there is no sign of chemical bonding among the phases in conventional $\mathrm{CaPO}_{4} /$ collagen biocomposites, probably due to a lack of suitable interfacial bonding during mixing [703]. However, this is not the case for phosphorylated collagens [690]. Readers skilled in computer modeling are forwarded to simulations of the interactions between collagen peptides and HA surfaces [1058] as well as between three polymers (PE, PA and PLA) and HA surfaces [1059], respectively.

To study possible interactions among the phases, Fourier-transformed infrared (FTIR) spectra of some $\mathrm{CaPO}_{4}$-based biocomposites and collagen films were collected and transformed into absorption spectra using the Kramers-Kronig equation to demonstrate energy shifts of residues on the apatite/collagen interface. After comparing FTIR spectra of biocomposites and collagen films in detail, red shifts of the absorption bands for $\mathrm{C}-\mathrm{O}$ bonds were observed in the spectra of the biocomposites. These red shifts were described as a decrease of bonding energies of $\mathrm{C}-\mathrm{O}$ bonds and assumed to be caused by an interaction to $\mathrm{Ca}^{2+}$ ions located on the surfaces of apatite nano-sized crystals, as shown in Figure 9 [688]. Another proof of a chemical interaction between apatite and collagen was also evaluated in FTIR spectra of CDHA/collagen biocomposites, in which a shift of the band corresponding to - $\mathrm{COO}^{-}$ stretching from 1340 to $1337 \mathrm{~cm}^{-1}$ was observed [654,655]. More to the point, nucleation of apatite crystals onto collagen through a chemical interaction with carboxylate groups of collagen macromolecules has been reported [1060-1062].

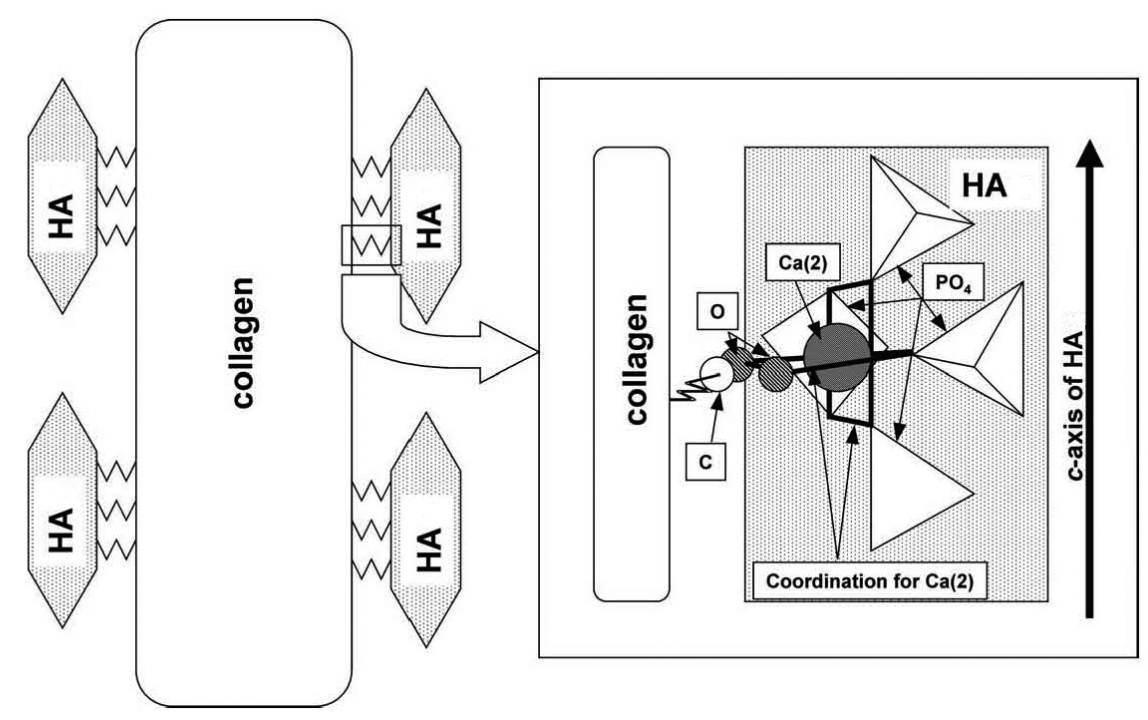

Figure 9. A schematic drawing of the relation between self-organization (directional deposition of HA on collagen) and interfacial interaction in biocomposites. Direction of interaction between HA and collagen is restricted by covalent bond between $\mathrm{COO}$ and $\mathrm{Ca}(2)$ to maintain regular coordination number of 7 . Reprinted from Ref. [688] with permission. 
FTIR spectroscopy seems to be the major tool to study a possible chemical bonding among the phases in $\mathrm{CaPO}_{4}$-based biocomposites and hybrid biomaterials [212,243,276,283,284,379,437,542,555,568, $571,573,577,580,591,612,617,620,628,655,690,737,738,777,806,835,1063-1069]$. For example, the characteristic bands at 2918, 2850 and $1472 \mathrm{~cm}^{-1}$ for the hydrocarbon backbone of PE appeared to have zero shift in an HA/PE biocomposite. However, in the case of PA, several FTIR bands indicated that the polar groups shifted apparently: the bands at 3304, 1273 and $692 \mathrm{~cm}^{-1}$ derived from stretching of $\mathrm{N}-\mathrm{H}$, stretching of $\mathrm{C}-\mathrm{N}-\mathrm{H}$ and vibrating of $\mathrm{N}-\mathrm{H}$ moved to 3306,1275 and $690 \mathrm{~cm}^{-1}$, respectively, in the HA/PA biocomposites. Furthermore, both stretching $\left(3568 \mathrm{~cm}^{-1}\right)$ and vibrating $\left(692 \mathrm{~cm}^{-1}\right)$ modes of hydroxide in HA moved to 3570 and $690 \mathrm{~cm}^{-1}$ in the HA/PA biocomposites, respectively, indicating the formation of hydrogen bonds. Besides, bands at 1094 and $1031 \mathrm{~cm}^{-1}$ of $\mathrm{PO}_{4}$ modes also shifted to 1093 and $1033 \mathrm{~cm}^{-1}$ in HA/PA biocomposites. The bands shift in a fingerprint area indicated that the hydroxide and orthophosphate on the surface of HA might interact with plentiful carboxyl and amino groups of PA through nucleophilic addition [212]. Comparable conclusions were made for HA/PVA [577], CDHA/alginate [655], ACP/PPF [437], HA/maleic anhydride [284], HA/carboxylated PU [1069] and $\beta$-TCP/PLLA [379] biocomposites, in which weak chemical bonds were considered to form between $\mathrm{Ca}^{2+}$ ions located on the HA, CDHA, ACP or $\beta$-TCP surface, respectively, and slightly polarized $\mathrm{O}$ atoms of $\mathrm{C}=\mathrm{O}$ bonds in the surrounding bioorganic compounds. The data obtained suggested that crystallization of $\mathrm{CaPO}_{4}$ in chitosan-containing solutions was substantially modulated by a chemical interaction of the components; apparently, a part of calcium was captured by chitosan and did not participate in the formation of the main mineral phase [1068]. Schematically, this type of the chemical interaction is shown in Figure 10 [655].

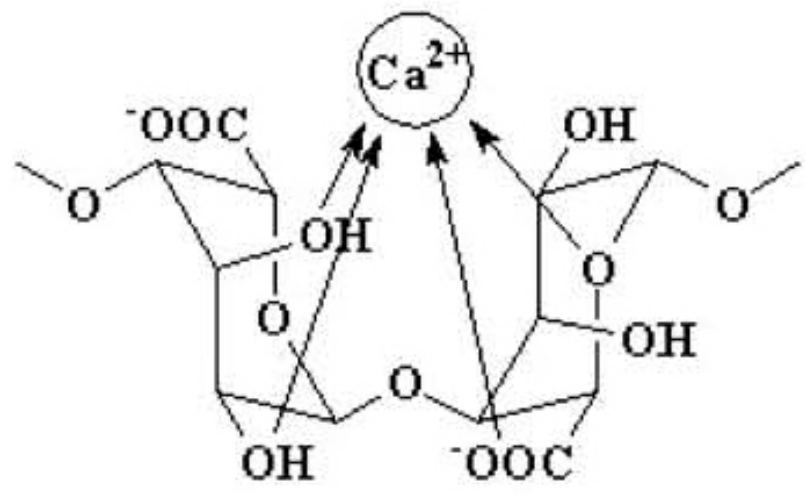

Figure 10. A schematic diagram of $\mathrm{Ca}^{2+}$ ion binding with alginate chains. Reprinted from Ref. [655] with permission.

Except of FTIR spectroscopy, other measurement techniques are also able to show some evidences of a chemical interaction among the phases in $\mathrm{CaPO}_{4}$-based biocomposites and hybrid biomaterials [276,379,571,573,577,1064-1071]. For example, for nano-sized crystals of CDHA/alendronate such evidences were observed by thermogravimetric analysis: DTG plots of the crystals appeared to be quite different from those obtained from mechanical mixtures of CDHA and calcium alendronate with similar compositions [1070]. Analogous DTG results were obtained for nano-sized HA/PVA biocomposites [577]. In the case of biocomposites of nano-sized HA with PA, a hydrogen bonding among the phases 
was detected by differential scanning calorimetry technique [571]. Similar results were obtained in another study [1065]. One more example comprises application of the dynamic mechanical analysis to investigate softening mechanism of $\beta$-TCP/PLLA biocomposites [379]. As to biocomposites of nano-sized HA with PVAP, some indirect evidences of a chemical bonding among the phases were found by X-ray diffraction and thermogravimetric analysis [276]. A strong structural correlation between the orientation of FA crystallites and gelatin within the FA/gelatin composite spheres was discovered that indicated to a substantial reorganization of the macromolecular matrix within the area of a growing aggregate [366]. In addition, chemical interactions between HA and organic molecules have been elucidated using ab initio calculation methods [1072].

By means of the X-ray photo-electronic spectroscopy (XPS) technique, binding energies of Ca, $\mathrm{P}$ and $\mathrm{O}$ atoms were found to have some differences between nano-sized HA (Ca: 350.5 and 345.5; O: 530.2; P: $132.5 \mathrm{eV}$ ) and nano-sized HA/konjac glucomannan/chitosan biocomposite (Ca: 352.1 and 347.4; O: 531.2; P: $133.4 \mathrm{eV}$, respectively) [591]. Further measurements by FTIR and X-ray diffraction revealed that nano-sized HA was mainly linked with konjac glucomannan and chitosan by hydrogen bonding among $\mathrm{OH}^{-}$and $\mathrm{PO}_{4}{ }^{3-}$ ions of $\mathrm{HA}$ and $-\mathrm{C}=\mathrm{O}$ and $-\mathrm{NH}$ groups of konjac glucomannan and chitosan copolymer and there was a stable interface formed among the three phases in the biocomposite. Meanwhile, coordinate bonding might be formed between $\mathrm{Ca}^{2+}$ and $-\mathrm{NH}$. Stable interfaces have been formed among the three phases in a biocomposite [591]. Hydrogen bonding between $\mathrm{C}=\mathrm{O}$ groups of PDLLA and the surface P-OH groups of HA was discovered by XPS in another study [1065]. In $\mathrm{HA} /$ collagen biocomposites, a covalent bond formation between $\mathrm{Ca}^{2+}$ ions of $\mathrm{HA}$ and $\mathrm{RCOO}^{-}$groups of collagen molecules was found by XPS [543]. Similar XPS observations were also made for several other $\mathrm{CaPO}_{4}$-based biocomposites and hybrid biomaterials [568,612,617]. All possible chemical interactions between HA crystals and bioorganic molecules in HA/chitosan-gelatin network films is shown in Figure 11 [1067].

The interaction and adhesion between $\mathrm{CaPO}_{4}$ fillers and respective matrixes have a significant effect on the properties of particulate filled reinforced materials, being essential to transfer the load among the phases and thus improve the mechanical performance of the biocomposites [283]. However, for a substantial amount of the aforementioned formulations, the interaction among the phases is mechanical in nature. This is because the matrix often consists of compounds with no functional groups or unsaturated bonds, which can form ionic complexes with the constituents of $\mathrm{CaPO}_{4}$. Obviously, less coupling exists between non-polar polymers and $\mathrm{CaPO}_{4}$ ceramic particles. Therefore, polymers with functional groups pendant to the polymer backbone, which can act as sites for bridging to $\mathrm{CaPO}_{4}$, are more promising in this respect [322].

In order to influence the interactions among the phases, various supplementary reagents are applied. Namely, if the primary effect of a processing additive is to increase the interaction between the phases, such additives can be regarded as coupling agents [1073]. These agents establish chemical bridges between the matrix and the fillers, promoting adhesion among the phases. In many cases, their effect is not unique; for example, it also might influence rheology of the composites [219]. In the case of $\mathrm{CaPO}_{4}$, a hexamethylene diisocyanate coupling agent was used to bind PEG/PBT (Polyactive ${ }^{\mathrm{TM}}$ ) block copolymers [228] and other polymers [1063] to HA filler particles. Thermogravimetric and infrared analysis demonstrated that the polymers were chemically bonded to the HA particles through the isocyanate groups, making it a suitable approach to improve the adhesion [1063]. Other researchers used 
glutaraldehyde as a cross-linked reagent [390,540,542,543,562,648,678,716,720,1074]. In addition, the interfacial bonding among $\mathrm{CaPO}_{4}$ and other components might be induced by silanes $[204,205,228$, 333,576,1075-1080], zirconates [219,333,335,1081-1083], titanates [219,333,1081,1084], phosphoric acid [579], alkaline pretreatment [799,801], PAA [1085] and some other chemicals. Furthermore, some polymers might be grafted onto the surface of $\mathrm{CaPO}_{4}$ particles [596]. Structural modifications of the polymeric matrices, for instance, with introduction of acrylic acid [186,204,205,228], have also proved to be effective methods. For example, application of polyacids as a bonding agent for HA/Polyactive ${ }^{\mathrm{TM}}$ composites caused the surface modified HA particles to maintain better contact with the polymer at fracture and improved mechanical properties [228]. The use of titanate and zirconate coupling agents appeared to be very dependent on the molding technique employed [219]. Silane-coupled HA powders were tested before applying them as fillers in biodegradable composites. This treatment allowed HA withstanding the attack of aqueous solutions without impairing overall bioactivity [1076-1080]. Besides, a chemically modified reinforcement phase-matrix interface was found to improve the mechanical properties of the biocomposites. The examples include chemically coupled HA/PE [204,205], chemically formed HA/Ca poly(vinylphosphonate) [280] and PLA/HA fibers [178]. These biocomposites are able to consume a large amount of energy in the fracture.

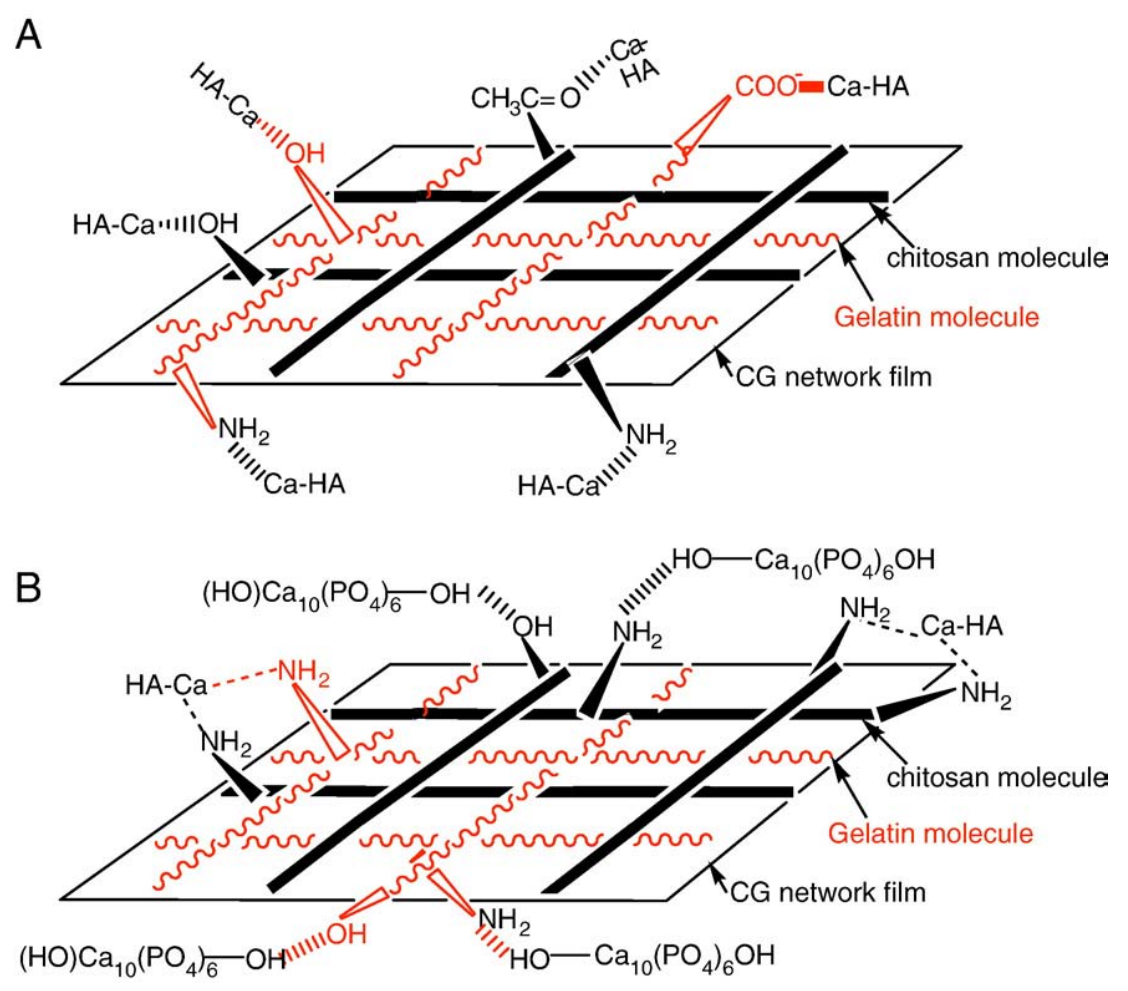

Figure 11. Possible interactions between a chitosan-gelatin (CG) network and HA crystals in HA/CG biocomposites: (A) In the case of a nano-dimensional HA (nHA); (B) In the case of a micro-dimensional HA (mHA). According to the authors: "When nHA formed on the surface of CG network via biomineralization, the corresponding ion interaction is the main drive force. However, as the mHA crystals depositing on the surface of $\mathrm{CG}$ network, the hydrogen bonds between $\mathrm{COOH}, \mathrm{OH},-\mathrm{NH}_{2}$ of $\mathrm{CG}$ films and $\mathrm{OH}$ groups of HA crystals take the important role.” (p. 1215). Reprinted from Ref. [1067] with permission. 
The action of some coupling agents was found to combine two distinct mechanisms: (i) crosslinking of the polymeric matrix (valid for zirconate and titanate coupling agents) and (ii) improvement of the interfacial interactions among the major phases of the biocomposites. This interfacial adhesion improvement appeared to be much dependent on the chemical nature ( $\mathrm{pH}$ and type of metallic centre) of the coupling agents [333]. Several works claimed that silanes did interact with HA [204,269,1076-1078]. It was shown that a silicon-containing inter-phase existed between HA and PE, which promoted the chemical adhesion between the HA particles and the polymer. A silane-coupling agent also facilitated penetration of PE into cavities of individual HA particles, which resulted in enhanced mechanical interlocking at the matrix-reinforcement interface [204,205].

Thus, the optimization of biocomposite properties by coupling agents is currently an important area of the research. The control and development of molecular-level associations of polymers with $\mathrm{CaPO}_{4}$ is suggested to be significant for the resulting mechanical responses in biocomposites. It appears that a fundamental molecular understanding of the interfacial behavior in biocomposites is an area not sufficiently addressed in literature. Various experimental characterization techniques using electron microscopy, vibrational spectroscopy, X-ray diffraction, scanning probe microscopy and others are used routinely to characterize these materials besides mechanical property characterization. In addition, atomic scale models for simulating the phase interaction and predicting responses in the novel material systems, where nanostructures and nano-interfaces are included, are important to understand and predict the load deformation behavior [1047].

In addition to the aforementioned, the surface of $\mathrm{CaPO}_{4}$ might be modified as well [596,1081-1093]. An interesting approach for HA surface modification was described [1092]. First, in situ synthesis of surface thiol-functionalized HA (HA-SH) was realized by adding 3-mercaptopropionic acid during hydrothermal synthesis of HA (Figure 12A). This was followed by grafting polymerization of ethylene glycol methacrylate phosphate by radical chain transfer generating the sulfur-centered radicals on the HA surfaces (Figure 12B), which initiated the surface grafting polymerization of ethylene glycol methacrylate phosphate (Figure 12C) [1092]. In certain cases, the surface functionalization of $\mathrm{CaPO}_{4}$ particles was found to decrease the bacterial adherence on their surface [1093]. Other examples might be found in literature [596,1081-1093]. In general, the purpose of surface modifying is not only to guarantee the even distribution of $\mathrm{CaPO}_{4}$ particles at a high loading level in the matrix but also to prevent or delay the debonding process of $\mathrm{CaPO}_{4}$ particles from the matrix. Obviously, all surface modifiers must satisfy several biomedical requirements, such as no toxicity, good biocompatibility and no changes in the biological or physico-chemical properties of the fillers.

Addition of adhesion promoting agents might be an alternative to improve the interaction between the fillers and the matrix. For example, Morita et al. incorporated 4-methacryloyloxyethyl trimellitate anhydride to promote adhesion of the polymer to HA [1094]. In another study, a phosphoric ester was added to the liquid component of the formulation [1095]. Both the strength and the affinity index of biocomposites were found to increase, probably due to the effects of co-polymerization.

Possible interactions between BCP and HPMC have been investigated in IBS composites $[838,839,1096]$. After mixing, there was a decrease in the mean diameter of BCP granules and this influenced the viscosity of the paste. Dissolution of grain boundaries of $\beta$-TCP crystals and precipitation of CDHA on HA crystal surface were found during the interaction. Both phenomena were responsible for the observed 
granulometric changes [838,839]; however, within the sensitivity of the employed measurement techniques, no chemical bonding between BCP and HPMC was detected [1096].

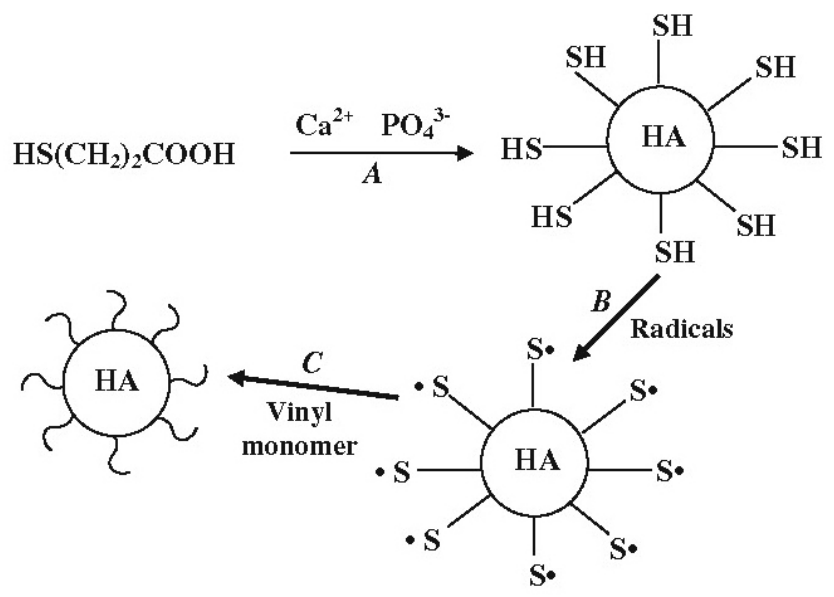

Figure 12. Surface modification of HA particles by grafting polymerization according to Lee et al., [1092]: (A) Surface thiol functionalized HA; (B) Sulfur-centered radical on HA surface; (C) Surface grafting polymerization of ethylene glycol methacrylate phosphate. Reprinted from Ref. [63] with permission.

A co-precipitation technique was used to prepare CDHA/chitosan biocomposites [746]. Growth of CDHA crystals was inhibited by organic acids with more than two carboxyl groups, which strongly bind to CDHA surfaces via COO-Ca bonds. Transmission electron microscopy images revealed that CDHA formed elliptic aggregates with chemical interactions (probably coordination bond) between $\mathrm{Ca}$ on its surface and amino groups of chitosan; the nano-sized crystals of CDHA were found to align along the chitosan molecules, with the amino groups working as the nucleation sites [746]. Formation of calcium cross-linked polymer carboxylate salts was suggested during setting of self-hardening (TTCP + DCPA)/polyphosphazane biocomposites; a chemical involvement of the polymer in the setting process was concluded based on the results of $\mathrm{pH}$ monitoring [490-492].

A chemical bond between the phases was presumed in PCL/HA composites, prepared by the grafting technique [345]; unfortunately, no strong experimental evidences were provided. In another study, $\mathrm{CDHA} /$ poly $(\alpha$-hydroxyester) composites were prepared by a low temperature chemical route [320]. In that study, pre-composite structures were prepared by combining $\alpha$-TCP with PLA, PLGA and copolymers thereof. The final biocomposite was achieved by in situ hydrolysis of $\alpha$-TCP to CDHA performed at $56{ }^{\circ} \mathrm{C}$ either in solvent cast or pressed pre-composites. That transformation occurred without any chemical reaction between the polymer and $\mathrm{CaPO}_{4}$, as it was determined by FTIR spectroscopy [320].

In nearly every study on HA/carbon nanotubes biocomposites, the nanotubes were functionalized before combining them with HA. Most researchers did this by oxidation [239-241,980,981], although non-covalent functionalizing with sodium dodecylsulfate [980] and coating the nanotubes by a polymer [1097] before combining them with HA were also reported. Several studies by transmission 
electron microscopy revealed evidences that the functionalization enhanced interaction between carbon nanotubes and HA [980,981,1098].

For $\mathrm{CaPO}_{4}$-based biocomposites able to sustain a high-temperature sintering (valid for the formulations consisting of inorganic components only), an inter-diffusion of chemical elements might take place among the phases. Such effect was detected by energy-dispersive X-ray spectroscopy in $\mathrm{HA} / \mathrm{TiO}_{2}$ biocomposite particles with partial formation of calcium titanates; this process was found to be favorable to enhancing the cohesive strength of particles in the composite coating [1099]. Similar high-temperature interactions between $\mathrm{HA}$ and zirconia [849,872], as well as between $\mathrm{HA}$ and $\mathrm{Ti}$ $[603,927,928,930,939,942]$ were also detected. Namely, lower Ti content composites sintered at $1200{ }^{\circ} \mathrm{C}$ showed main crystalline phases as $\mathrm{CaTiO}_{3}, \mathrm{CaO}$ and $\mathrm{Ti}_{x} \mathrm{P}_{y}$, while an increase in Ti content to 50 vol.\% revealed $\mathrm{Ti}_{2} \mathrm{O}$ and residual $\alpha$-Ti as additional phases. Thus, the chemical interactions between HA and Ti were expressed by the following unbalanced illustrative scheme [928]:

$$
\mathrm{Ti}+\mathrm{Ca}_{10}\left(\mathrm{PO}_{4}\right)_{6}(\mathrm{OH})_{2} \rightarrow \mathrm{CaTiO}_{3}+\mathrm{CaO}+\mathrm{Ti}_{\mathrm{x}} \mathrm{P}_{\mathrm{y}}+\left(\mathrm{Ti}_{2} \mathrm{O}\right)+\left(\mathrm{Ca}_{4} \mathrm{P}_{2} \mathrm{O}_{9}\right)+\mathrm{H}_{2} \mathrm{O}
$$

Such undesired interactions between Ti and HA could be minimized if Ti particles were coated by silica [943].

Besides, partial decomposition of HA and formation of various calcium aluminates were detected in $\mathrm{HA} / \mathrm{Al}_{2} \mathrm{O}_{3}$ biocomposites after sintering at $1200-1300{ }^{\circ} \mathrm{C}$. This has been attributed to the diffusion of $\mathrm{Ca}^{2+}$ from HA into the alumina matrix and the depletion of $\mathrm{Ca}^{2+}$ from $\mathrm{HA}$ leads to the decomposition of HA into $\beta$-TCP. All these processes influence the mechanical strength of the biocomposites [883-887,1099].

\section{Bioactivity and Biodegradation}

The continuous degradation of an implant causes a gradual load transfer to the healing tissue, preventing stress-shielding atrophy and stimulates the healing and remodeling of bones. Some requirements must be fulfilled by the ideal prosthetic biodegradable materials, such as biocompatibility, adequate initial strength and stiffness, retention of mechanical properties throughout sufficient time to assure its biofunctionality and non-toxicity of the degradation by-products [125]. In most cases, bioactivity (i.e., ability of bonding to bones) of biologically relevant $\mathrm{CaPO}_{4}$ reinforced by other materials is lower than that of pure $\mathrm{CaPO}_{4}[148,1100]$.

In general, both bioactivity and biodegradability of any biocomposite and/or hybrid biomaterial are determined by the same properties of the constituents. Both processes are very multi-factorial because, during implantation, the surface of any graft contacts with biological fluids and, shortly afterwards, is colonized by cells. Much more biology, than chemistry and material science altogether, is involved into these very complex processes and many specific details still remain unknown. In addition, biodegradation of all components of biocomposites occurs simultaneously and the obtained byproducts might influence both the entire process and biodegradation of each component. For example, in the case of biocomposites prepared from polyesters and TCP, hydrolysis reactions of the ester bonds, acid dissociation of the carboxylic end groups, dissolution of TCP and buffering reactions by the dissolved phosphate ions occur simultaneously [1101-1104]. In such cases, basic TCP buffer the acidic degradation products of polyesters, thus reducing autocatalysis and delaying polymer degradation. This is why both $\mathrm{pH}$ and mass drops occurred at earlier degradation time points for the pure polymer samples 
than for the corresponding composites. However, this is not always the case. Namely, studies are available, in which the presence of $\mathrm{CaPO}_{4}$ did not have an effect on the degradation rate of the polymer matrix [1105-1107]. Therefore, to simplify the task, biodegradation of the individual components should be considered independently. Namely, an in vitro biodegradation of the biologically relevant $\mathrm{CaPO}_{4}$ might be described by their chemical dissolution in slightly acidic media (they are almost insoluble in alkaline solutions [74,75]), which, in the case of CDHA, might be described as a sequence of four successive chemical Equations (2)-(5) [1108,1109]:

$$
\begin{aligned}
& \mathrm{Ca}_{10-x}\left(\mathrm{HPO}_{4}\right)_{x}\left(\mathrm{PO}_{4}\right)_{6-x}(\mathrm{OH})_{2-x}+(2-x) \mathrm{H}^{+}=\mathrm{Ca}_{10-x}\left(\mathrm{HPO}_{4}\right)_{x}\left(\mathrm{PO}_{4}\right)_{6-x}\left(\mathrm{H}_{2} \mathrm{O}\right)_{2-x}(2-x)^{+} \\
& \mathrm{Ca}_{10-x}\left(\mathrm{HPO}_{4}\right)_{x}\left(\mathrm{PO}_{4}\right)_{6-x}\left(\mathrm{H}_{2} \mathrm{O}\right)_{2-x}\left(2_{-}\right)^{+}= 3 \mathrm{Ca}_{3}\left(\mathrm{PO}_{4}\right)_{2}+(1-x) \mathrm{Ca}^{2+}+(2-x) \mathrm{H}_{2} \mathrm{O} \\
& \mathrm{Ca}_{3}\left(\mathrm{PO}_{4}\right)_{2}+2 \mathrm{H}^{+}=\mathrm{Ca}^{2+}+2 \mathrm{CaHPO}_{4} \\
& \mathrm{CaHPO}_{4}+\mathrm{H}^{+}=\mathrm{Ca}^{2+}+\mathrm{H}_{2} \mathrm{PO}_{4}^{-}
\end{aligned}
$$

Biodegradability of polymers generally depends on the following factors: 1) chemical stability of the polymer backbone, 2) hydrophobicity of the monomer, 3) morphology of the polymer, 4) initial molecular weight, 5) fabrication processes, 6) geometry of the implant, 7) properties of the scaffold such as porosity and pore diameter [262]. A summary on degradation of PLA and PGA, as well as that of SEVA-C is available in literature Ref. [125] (p. 798 and p. 803, respectively), where the interested readers are referred to.

Concerning in vivo studies, biodegradation of HA/PLLA and CDHA/PLLA biocomposite rods in subcutis and medullary cavities of rabbits were investigated mechanically and histologically; the degradation was found to be faster for the case of using uncalcinated CDHA instead of calcinated HA [1110]. In a more detailed study, new bone formation was detected at 2 weeks after implantation, especially for formulations with a high HA content [1111]. More to the point, a direct contact between bones and these composites without intervening fibrous tissue was detected in this case $[1111,1112]$. Both SEVA-C and SEVA-C/HA biocomposites were found to exhibit a non-cytotoxic behavior $[1113,1114]$, inducing a satisfactory tissue response when implanted as shown by in vivo studies [1114]. Furthermore, SEVA-C/HA biocomposites induced a positive response on osteoblast-like cells to what concerned cell adhesion and proliferation [1113]. An in vivo study on biodegradation of microspheres (PLGA, gelatin and PTMC were used) $/ \mathrm{CaPO}_{4}$ biocomposites revealed that they exhibited microsphere degradation after 12 weeks of subcutaneous implantation, which was accompanied by compression strength decreasing [1115]. Interestingly that the amount of $\mathrm{CaPO}_{4}$ in biocomposites was found to have a greater effect on the early stages of osteoblast behavior (cell attachment and proliferation) rather than the immediate and late stages (proliferation and differentiation) [1116].

Both in vitro (the samples were immersed into $1 \%$ trypsin/phosphate-buffered saline solution at $37^{\circ} \mathrm{C}$ ) and in vivo (implantation of samples into the posterolateral lumbar spine of rabbits) biodegradation were investigated for nano-sized HA/collagen/PLA biocomposites [551]. The results demonstrated that weight loss increased continuously in vitro with a reduction in mass of $\sim 20 \%$ after 4 weeks. During the experimental period in vitro, a relative rate of reduction of the three components in this material was shown to differ greatly: collagen decreased the fastest, from $40 \%$ by weight to $20 \%$ in the composite; HA content increased from $45 \%$ to $\sim 60 \%$; while the amount of PLA changed a little. In vivo, the collagen/HA ratio appeared to be slightly higher near the transverse process than in the 
central part of the intertransverse process [551]. Hasegawa et al., [1117] performed in vivo study, spanning over a period of 5-7 years, on high strength HA/PLLA biocomposite rods for the internal fixation of bone fractures. In that work, both uncalcined CDHA and calcined HA were used as reinforcing phases in PLLA matrix. Those composites were implanted in the femur of 25 rabbits. It was found that the implanted materials were resorbed after 6 years of implantation. The presence of remodeled bone and trabecular bone bonding was the significant outcome. These data clearly demonstrate a biodegradation independence of various components of biocomposites.

\section{Some Challenges and Critical Issues}

The scientific information summarized in this review represents the developments of $\mathrm{CaPO}_{4}$-based biocomposites and hybrid biomaterials from a variety of approaches, starting from conventional ones to tissue engineering. Such formulations combined with osteoconductive, osteoinductive factors, and/or osteogenic cells have already gained much interest as a new and versatile class of biomaterials and are perceived to be beneficial in many aspects as bone grafts [22,1118]. However, current applications of these biomaterials in medicine and surgery are still remarkably less than might be expected. In many biomedical applications, research and testing of such formulations have been introduced and highly developed but only in a very few cases an industrial production and commercial distribution of medical devices partially or entirely made of biocomposites have started. The medical application of biocomposites and hybrid biomaterials requires a better understanding of the objectives and limitations involved. Recently, the main critical issues have been summarized as follows [197]:

- There are not enough reliable experimental and clinical data supporting the long-term performance of biocomposites with respect to monolithic traditional materials;

- The design of biocomposites and hybrid biomaterials is far more complex than that of conventional monolithic materials because of the large number of additional design variables to be considered;

- The available fabrication methods may limit the possible reinforcement configurations, may be time consuming, expensive, highly skilled and may require special cleaning and sterilization processes;

- There are no satisfactory standards yet for biocompatibility testing of the biocomposite implants because the ways in which the components of any biocomposite interact to living tissues are not completely understood;

- There are no adequate standards for the assessment of biocomposite fatigue performance because the fatigue behavior of such materials is far more complex and difficult to predict than that of traditional materials [197].

In addition, much work needs to be done in the analysis of cells and their different behaviors with regard to their interactions with $\mathrm{CaPO}_{4}$ /polymer biocomposites. Important but unresolved questions are: (1) What is/are the mechanism(s) by which such biocomposites promote cell proliferation and differentiation? (2) How can we determine the pathways? Future studies will focus on the ability to functionalize the surfaces of $\mathrm{CaPO}_{4} /$ polymer biocomposites with molecules of different natures and 
dimensions by means of their attachment to cells that will enable them to act selectively on biological molecules such as proteins and peptides [264].

On the other hand, in spite of an enormous progress in biocomposite processing, to achieve the desired characteristics researchers still need to develop more advanced technologies to fabricate a bone-resembling hierarchical organization over several length scales. Development of novel grafting materials depends on the progress in research into the structure of natural bones. The key issues are not only to understand the fundamentals of biomineralization but also to translate such knowledge into practical synthetic pathways to produce better bone grafts. Unfortunately, when it comes to the fabrication of biocomposites, mimicing natural bones from the nanometer to the micrometer dimensions, there are many key issues, including control of morphology, incorporation of foreign ions, interaction with biomolecules and assembly of the organic and inorganic phases, which are still not well understood. A processing gap between the lower-level building units and the higher-order architecture could severely limit the practical application of current $\mathrm{CaPO}_{4}$-based biocomposites and hybrid biomaterials. Therefore, further substantial research efforts have been outlined to address the following key challenges [22]:

- Optimizing biocomposite processing conditions;

- Optimization of interfacial bonding and strength equivalent to natural bone;

- Optimization of the surface properties and pore size to maximize bone growth;

- Maintaining the adequate volume of the construct in vivo to allow bone formation to take place;

- Withstanding the load-bearing conditions;

- Matching the bioresorbability of the grafts and their biomechanical properties while forming new bone;

- Understanding the molecular mechanisms by which the cells and the biocomposite matrix interact with each other in vivo to promote bone regeneration;

- Supporting angiogenesis and vascularization for the growth of healthy bone cells and subsequent tissue formation and remodeling.

The aforementioned critical issues have to be solved before a widespread commercial use of $\mathrm{CaPO}_{4}-$ based biocomposites and hybrid biomaterials can be made in surgery and medicine.

\section{Conclusions}

All types of calcified tissues of mammals appear to possess complex hierarchical structures and, from the material point of view, they are multi-phase organic/inorganic biocomposites. Their mechanical properties are outstanding (considering weak constituents from which they are assembled) and far beyond those, that can be achieved using the same synthetic materials with present technologies. This is because biological organisms produce biocomposites that are organized in terms of both composition and structure, containing both brittle $\mathrm{CaPO}_{4}$ and ductile bioorganic components in very complex structures, hierarchically organized at the nano-, micro- and meso-levels. Additionally, the calcified tissues are always multifunctional: for example, bones provide structural support for the body and are responsible for blood cell formation. The third defining characteristic of biological systems, in contrast with current synthetic systems, is their self-healing ability, which is nearly universal in nature. These 
complex structures, which have risen from millions of years of evolution, inspire materials scientists in the design of novel biomaterials [1119].

Obviously, no single-phase biomaterial is able to provide all the essential features of bones and/or other calcified tissues and therefore, there is a great need to engineer multi-phase biocomposites with the structure and composition mimicking those of natural bones. This resulted in an idea of adding $\mathrm{CaPO}_{4}$ to polymers, which further led from the bioinert monolithic materials used in the 1970s and 1980s to the bioactive biodegradable biocomposites used now as modulus-matched biomaterials and since recently as the scaffolds for tissue engineering. This approach resulted in applications of conventional composite manufacturing techniques to the field of biomaterials and the studies summarized in this review have shown that the proper combination of a ductile matrix with a brittle, hard and bioactive $\mathrm{CaPO}_{4}$ filler offers many advantages for biomedical applications. Namely, the desirable properties of some components can compensate for a poor mechanical behavior of $\mathrm{CaPO}_{4}$ bioceramics, while in turn the desirable bioactive properties of $\mathrm{CaPO}_{4}$ improve those of other phases, thus expanding the possible application of each material within the body $[66,67]$. However, the reviewed literature clearly indicates that among possible types of $\mathrm{CaPO}_{4}$-based biocomposites and hybrid biomaterials only simple, complex and graded ones, as well as fibrous, laminar and particulate ones (see Section 2. "General Information and Knowledge" for details) have been investigated. Presumably, a future progress in this subject will require concentrating efforts on elaboration and development of both hierarchical and hybrid biocomposites. Furthermore, following the modern tendency of tissue engineering, a novel generation of $\mathrm{CaPO}_{4}$-based biocomposites and hybrid biomaterials should also contain a biological living part.

To finalize, the future of the $\mathrm{CaPO}_{4}$-based biocomposites and hybrid biomaterials is now directly dependent on the formation of multidisciplinary teams composed of experts but primarily experts ready to collaborate in close collaboration with others and thus be able to deal efficiently with the complexity of the human organism. The physical chemistries of solids, solid surfaces, polymer dispersion and solutions, as well as material-cell interactions are among the phenomena to be tackled. Furthermore, much work remains to be done on a long way from a laboratory to clinics and the success depends on the effective co-operation of clinicians, chemists, biologists, bioengineers and materials scientists.

\section{Conflicts of Interest}

The author declares no conflict of interest.

\section{Abbreviations}

$\begin{array}{ll}\text { A-W } & \text { apatite-wollastonite } \\ \text { BMP } & \text { bone morphogenetic protein } \\ \text { BSA } & \text { bovine serum albumin } \\ \text { CMC } & \text { carboxymethylcellulose } \\ \text { EVOH } & \text { a copolymer of ethylene and vinyl alcohol } \\ \text { IBS } & \text { injectable bone substitute } \\ \text { HDPE } & \text { high-density polyethylene } \\ \text { HPMC } & \text { hydroxypropylmethylcellulose } \\ \text { PA } & \text { polyamide }\end{array}$




\begin{tabular}{|c|c|}
\hline PAA & polyacrylic acid \\
\hline PBT & polybutyleneterephthalate \\
\hline PCL & $\operatorname{poly}(\varepsilon$-caprolactone $)$ \\
\hline PDLLA & poly(D,L-lactic acid) \\
\hline $\mathrm{PE}$ & polyethylene \\
\hline PEEK & polyetheretherketone \\
\hline PEG & polyethylene glycol \\
\hline PGA & polyglycolic acid \\
\hline PHB & polyhydroxybutyrate \\
\hline PHBHV & poly(hydroxybutyrate-co-hydroxyvalerate) \\
\hline PHEMA & polyhydroxyethyl methacrylate \\
\hline PLA & polylactic acid \\
\hline PLGA & poly(lactic-co-glycolic) acid \\
\hline PLGC & $c o$-polyester lactide- $c o$-glycolide- $c o$ - $\varepsilon$-caprolactone \\
\hline PLLA & poly(L-lactic acid) \\
\hline PMMA & polymethylmethacrylate \\
\hline PP & polypropylene \\
\hline PPF & poly(propylene-co-fumarate) \\
\hline PSZ & partially stabilized zirconia \\
\hline PTMC & poly(trimethylene carbonate) \\
\hline PU & polyurethane \\
\hline PVA & polyvinyl alcohol \\
\hline PVAP & polyvinyl alcohol phosphate \\
\hline SEVA-C & a blend of EVOH with starch \\
\hline
\end{tabular}

\section{References}

1. Chau, A.M.T.; Mobbs, R.J. Bone graft substitutes in anterior cervical discectomy and fusion. Eur. Spine J. 2009, 18, 449-464.

2. Kaveh, K.; Ibrahim, R.; Bakar, M.Z.A.; Ibrahim, T.A. Bone grafting and bone graft substitutes. J. Anim. Vet. Adv. 2010, 9, 1055-1067.

3. Shibuya, N.; Jupiter, D.C. Bone graft substitute: allograft and xenograft. Clin. Podiatr. Med. Surg. 2015, 32, 21-34.

4. Conway, J.D. Autograft and nonunions: morbidity with intramedullary bone graft versus iliac crest bone graft. Orthop. Clin. North Am. 2010, 41, 75-84.

5. Li, S.; Chen, Y.; Lin, Z.; Cui, W.; Zhao, J.; Su, W. A systematic review of randomized controlled clinical trials comparing hamstring autografts versus bone-patellar tendon-bone autografts for the reconstruction of the anterior cruciate ligament. Arch. Orthop. Trauma Surg. 2012, 132, $1287-1297$.

6. Keller, E.E.; Triplett, W.W. Iliac crest bone grafting: review of 160 consecutive cases. J. Oral Maxillofac. Surg. 1987, 45, 11-14. 
7. Schaaf, H.; Lendeckel, S.; Howaldt, H.P.; Streckbein, P. Donor site morbidity after bone harvesting from the anterior iliac crest. Oral Surg. Oral Med. Oral Pathol. Oral Radiol. Endod. 2010, 109, 52-58.

8. Carlsen, A.; Gorst-Rasmussen, A.; Jensen, T. Donor site morbidity associated with autogenous bone harvesting from the ascending mandibular ramus. Implant Dent. 2013, 22, 503-506.

9. Qvick, L.M.; Ritter, C.A.; Mutty, C.E.; Rohrbacher, B.J.; Buyea, C.M.; Anders, M.J. Donor site morbidity with reamer-irrigator-aspirator (RIA) use for autogenous bone graft harvesting in a single centre 204 case series. Injury 2013, 44, 1263-1269.

10. Li, Z.; Kawashita, M. Current progress in inorganic artificial biomaterials. J. Artif. Organ. 2011, $14,163-170$.

11. Bojar, W.; Kucharska, M.; Ciach, T.; Koperski, Ł.; Jastrzębski, Z.; Szałwiński, M. Bone regeneration potential of the new chitosan-based alloplastic biomaterial. J. Biomater. Appl. 2014, $28,1060-1068$.

12. Panchbhavi, V.K. Synthetic bone grafting in foot and ankle surgery. Foot Ankle Clin. 2010, 15, 559-576.

13. Dinopoulos, H.; Dimitriou, R.; Giannoudis, P.V. Bone graft substitutes: What are the options? Surgeon 2012, 10, 230-239.

14. Weiner, S.; Wagner, H.D. The material bone: structure-mechanical function relations. Ann. Rev. Mater. Sci. 1998, 28, 271-298.

15. Rey, C.; Combes, C.; Drouet, C.; Glimcher, M.J. Bone mineral: update on chemical composition and structure. Osteoporos. Int. 2009, 20, 1013-1021.

16. Dorozhkin, S.V. Calcium Orthophosphates: Applications in Nature, Biology, and Medicine; Pan Stanford: Singapore, 2012; p. 854.

17. Dorozhkin, S.V. Calcium orthophosphate-based bioceramics. Materials 2013, 6, 3840-3942.

18. Burr, D.B. The contribution of the organic matrix to bone's material properties. Bone 2002, 31, $8-11$.

19. Fratzl, P.; Gupta, H.S.; Paschalis, E.P.; Roschger, P. Structure and mechanical quality of the collagen-mineral nano-composite in bone. J. Mater. Chem. 2004, 14, 2115-2123.

20. Olszta, M.J.; Cheng, X.G.; Jee, S.S.; Kumar, B.R.; Kim, Y.Y.; Kaufman, M.J.; Douglas, E.P.; Gower, L.B. Bone structure and formation: a new perspective. Mater. Sci. Eng. $R$ 2007, 58, 77-116.

21. Fonseca, H.; Moreira-Gonçalves, D.; Coriolano, H.J.A.; Duarte, J.A. Bone quality: The determinants of bone strength and fragility. Sports Med. 2014, 44, 37-53.

22. Murugan, R.; Ramakrishna, S. Development of nanocomposites for bone grafting. Compos. Sci. Technol. 2005, 65, 2385-2406.

23. Suchanek, W.; Yoshimura, M. Processing and properties of hydroxyapatite-based biomaterials for use as hard tissue replacement implants. J. Mater. Res. 1998, 13, 94-117.

24. Vallet-Regi, M.; Arcos, D. Nanostructured hybrid materials for bone tissue regeneration. Curr. Nanosci. 2006, 2, 179-189.

25. Doblaré, M.; Garcia, J.M.; Gómez, M.J. Modelling bone tissue fracture and healing: a review. Eng. Fract. Mech. 2004, 71, 1809-1840.

26. Vallet-Regi, M. Revisiting ceramics for medical applications. Dalton Trans. 2006, 5211-5220. 
27. Pioletti, D.P. Biomechanics in bone tissue engineering. Comput. Methods Biomech. Biomed. Engin. 2010, 13, 837-846.

28. Huiskes, R.; Ruimerman, R.; van Lenthe, H.G.; Janssen, J.D. Effects of mechanical forces on maintenance and adaptation of form in trabecular bone. Nature 2000, 405, 704-706.

29. Boccaccini, A.R.; Blaker, J.J. Bioactive composite materials for tissue engineering scaffolds. Expert Rev. Med. Dev. 2005, 2, 303-317.

30. Hutmacher, D.W.; Schantz, J.T.; Lam, C.X.F.; Tan, K.C.; Lim, T.C. State of the art and future directions of scaffold-based bone engineering from a biomaterials perspective. J. Tissue Eng. Regen. Med. 2007, 1, 245-260.

31. Guarino, V.; Causa, F.; Ambrosio, L. Bioactive scaffolds for bone and ligament tissue. Expert Rev. Med. Dev. 2007, 4, 405-418.

32. Yunos, D.M.; Bretcanu, O.; Boccaccini, A.R. Polymer-bioceramic composites for tissue engineering scaffolds. J. Mater. Sci. 2008, 43, 4433-4442.

33. Zhao, H.X. Progress of study on drug-loaded chitosan/hydroxyapatite composite in bone tissue engineering. J. Funct. Mater. 2014, 45, 13006-13012, 13020.

34. Hench, L.L.; Polak, J.M. Third-generation biomedical materials. Science 2002, 295, 1014-1017.

35. Mathijsen, A. Nieuwe Wijze van Aanwending van het Gips-Verband bij Beenbreuken; J.B. van Loghem: Haarlem, The Netherlands, 1852; p. 21.

36. Dreesman, H. Über Knochenplombierung. Beitr. Klin. Chir. 1892, 9, 804-810.

37. Wang, M. Developing bioactive composite materials for tissue replacement. Biomaterials 2003, 24, 2133-2151.

38. Composite material. Available online: http:/en.wikipedia.org/wiki/Composite_material (assessed on 16 June 2015).

39. Gibson, R.F. A review of recent research on mechanics of multifunctional composite materials and structures. Compos. Struct. 2010, 92, 2793-2810.

40. Evans, S.L.; Gregson, P.J. Composite technology in load-bearing orthopaedic implants. Biomaterials 1998, 19, 1329-1342.

41. Wan, Y.Z.; Hong, L.; Jia, S.R.; Huang, Y.; Zhu, Y.; Wang, Y.L.; Jiang, H.J. Synthesis and characterization of hydroxyapatite-bacterial cellulose nanocomposites. Compos. Sci. Technol. 2006, 66, 1825-1832.

42. Wan, Y.Z.; Huang, Y.; Yuan, C.D.; Raman, S.; Zhu, Y.; Jiang, H.J.; He, F.; Gao, C. Biomimetic synthesis of hydroxyapatite/bacterial cellulose nanocomposites for biomedical applications. Mater. Sci. Eng. C 2007, 27, 855-864.

43. Ohtsuki, C.; Kamitakahara, M.; Miyazaki, T. Coating bone-like apatite onto organic substrates using solutions mimicking body fluid. J. Tissue Eng. Regen. Med. 2007, 1, 33-38.

44. Oyane, A. Development of apatite-based composites by a biomimetic process for biomedical applications. J. Ceram. Soc. Jpn. 2010, 118, 77-81.

45. Dorozhkin, S.V. Calcium orthophosphate deposits: preparation, properties and biomedical applications. Mater. Sci. Eng. C 2015, 55, 272-326.

46. Surmenev, R.A.; Surmeneva, M.A.; Ivanova, A.A. Significance of calcium phosphate coatings for the enhancement of new bone osteogenesis-A review. Acta Biomater. 2014, 10, 557-579. 
47. Dorozhkin, S.V. Calcium orthophosphate coatings on magnesium and its biodegradable alloys. Acta Biomater. 2014, 10, 2919-2934.

48. Zhao, J.; Guo, L.Y.; Yang, X.B.; Weng, J. Preparation of bioactive porous HA/PCL composite scaffolds. Appl. Surf. Sci. 2008, 255, 2942-2946.

49. Dorozhkin, S.; Ajaal, T. Toughening of porous bioceramic scaffolds by bioresorbable polymeric coatings. Proc. Inst. Mech. Eng. H 2009, 223, 459-470.

50. Woo, A.S.; Jang, J.L.; Liberman, R.F.; Weinzweig, J. Creation of a vascularized composite graft with acellular dermal matrix and hydroxyapatite. Plast. Reconstr. Surg. 2010, 125, 1661-1669.

51. Zhao, J.; Duan, K.; Zhang, J.W.; Lu, X.; Weng, J. The influence of polymer concentrations on the structure and mechanical properties of porous polycaprolactone-coated hydroxyapatite scaffolds. Appl. Surf. Sci. 2010, 256, 4586-4590.

52. Dong, J.; Uemura, T.; Kojima, H.; Kikuchi, M.; Tanaka, J.; Tateishi, T. Application of lowpressure system to sustain in vivo bone formation in osteoblast/porous hydroxyapatite composite. Mater. Sci. Eng. C 2001, 17, 37-43.

53. Zerbo, I.R.; Bronckers, A.L.J.J.; de Lange, G.; Burger, E.H. Localisation of osteogenic and osteoclastic cells in porous $\beta$-tricalcium phosphate particles used for human maxillary sinus floor elevation. Biomaterials 2005, 26, 1445-1451.

54. Mikán, J.; Villamil, M.; Montes, T.; Carretero, C.; Bernal, C.; Torres, M.L.; Zakaria, F.A. Porcine model for hybrid material of carbonated apatite and osteoprogenitor cells. Mater. Res. Innov. 2009, 13, 323-326.

55. Oe, K.; Miwa, M.; Nagamune, K.; Sakai, Y.; Lee, S.Y.; Niikura, T.; Iwakura, T.; Hasegawa, T.; Shibanuma, N.; Hata, Y.; et al. Nondestructive evaluation of cell numbers in bone marrow stromal cell/ $\beta$-tricalcium phosphate composites using ultrasound. Tissue Eng. C 2010, 16, 347-353.

56. Krout, A.; Wen, H.B.; Hippensteel, E.; Li, P. A hybrid coating of biomimetic apatite and osteocalcin. J. Biomed. Mater. Res. A 2005, 73, 377-387.

57. Kundu, B.; Soundrapandian, C.; Nandi, S.K.; Mukherjee, P.; Dandapat, N.; Roy, S.; Datta, B.K.; Mandal, T.K.; Basu, D.; Bhattacharya, R.N. Development of new localized drug delivery system based on ceftriaxone-sulbactam composite drug impregnated porous hydroxyapatite: a systematic approach for in vitro and in vivo animal trial. Pharm. Res. 2010, 27, 1659-1676.

58. Kickelbick, G. Hybrid Materials. Synthesis, Characterization, and Applications; Wiley-VCH Verlag: Weinheim, Germany, 2007; p. 498.

59. Matthews, F.L.; Rawlings, R.D. Composite Materials: Engineering and Science; CRC Press: Boca Raton, FL, USA, 2000; p. 480.

60. Xia, Z.; Riester, L.; Curtin, W.A.; Li, H.; Sheldon, B.W.; Liang, J.; Chang, B.; Xu, J.M. Direct observation of toughening mechanisms in carbon nanotube ceramic matrix composites. Acta Mater. 2004, 52, 931-944.

61. Tavares, M.I.B.; Ferreira, O.; Preto, M.; Miguez, E.; Soares, I.L.; da Silva, E.P. Evaluation of composites miscibility by low field NMR. Int. J. Polym. Mater. 2007, 56, 1113-1118.

62. Kiran, E. Polymer miscibility, phase separation, morphological modifications and polymorphic transformations in dense fluids. J. Supercrit. Fluids 2009, 47, 466-483. 
63. Šupová, M. Problem of hydroxyapatite dispersion in polymer matrices: A review. J. Mater. Sci. Mater. Med. 2009, 20, 1201-1213.

64. Böstman, O.; Pihlajamäki, H. Clinical biocompatibility of biodegradable orthopaedic implants for internal fixation: A review. Biomaterials 2000, 21, 2615-2621.

65. John, M.J.; Thomas, S. Biofibres and biocomposites. Carbohydr. Polym. 2008, 71, 343-364.

66. Rea, S.M.; Bonfield, W. Biocomposites for medical applications. J. Aust. Ceram. Soc. 2004, 40, $43-57$.

67. Tanner, K.E. Bioactive ceramic-reinforced composites for bone augmentation. J. R. Soc. Interface 2010, 7, S541-S557.

68. Gravitis, Y.A.; Tééyaér, R.E.; Kallavus, U.L.; Andersons, B.A.; Ozol'-Kalnin, V.G.; Kokorevich, A.G.; Érin'sh, P.P.; Veveris, G.P. Biocomposite structure of wood cell membranes and their destruction by explosive autohydrolysis. Mech. Compos. Mater. 1987, 22, 721-725.

69. Bernard, S.L.; Picha, G.J. The use of coralline hydroxyapatite in a 'biocomposite' free flap. Plast. Reconstr. Surg. 1991, 87, 96-107.

70. Dorozhkin, S.V. Calcium orthophosphates and human beings. A historical perspective from the 1770s until 1940. Biomatter 2012, 2, 53-70.

71. Dorozhkin, S.V. A detailed history of calcium orthophosphates from 1770s till 1950. Mater. Sci. Eng. C 2013, 33, 3085-3110.

72. Hing, K.A. Bioceramic bone graft substitutes: influence of porosity and chemistry. Int. J. Appl. Ceram. Technol. 2005, 2, 184-199.

73. R. Naqshbandi, A.; Sopyan, I.; Gunawan. Development of porous calcium phosphate bioceramics for bone implant applications: a review. Rec. Pat. Mater. Sci. 2013, 6, 238-252.

74. LeGeros, R.Z. Calcium Phosphates in Oral Biology and Medicine. In Monographs in Oral Science; Myers, H.M., Ed.; Karger: Basel, Switzerland, 1991; Volume 15, p. 201.

75. Elliott, J.C. Structure and Chemistry of the Apatites and Other Calcium Orthophosphates. In Studies in Inorganic Chemistry; Agrawal, P.K., Ed.; Elsevier: Amsterdam, The Netherlands, 1994; Volume 18, p. 389.

76. Amjad, Z. Calcium Phosphates in Biological and Industrial Systems; Kluwer: Boston, MA, USA, 1997; p. 529.

77. Heimann, R.B. Calcium Phosphate: Structure, Synthesis, Properties, and Applications; Nova Science: New York, NY, USA, 2012; p. 498.

78. Gshalaev, V.S.; Demirchan, A.C. Hydroxyapatite: Synthesis, Properties and Applications; Nova Science: New York, NY, USA, 2012; p. 477.

79. Carraher, C.E., Jr. Introduction to Polymer Chemistry, 2nd ed.; CRC Press: Boca Raton, FL, USA, 2010; p. 534.

80. Young, R.J.; Lovell, P.A. Introduction to Polymers, 3rd ed.; CRC Press: Boca Raton, FL, USA, 2011; p. 688.

81. Thomson, R.C.; Ak, S.; Yaszemski, M.J.; Mikos, A.G. Polymer Scaffold Processing. In Principles of Tissue Engineering; Academic Press: New York, NY, USA, 2000; p. 251-262.

82. Ramakrishna, S.; Mayer, J.; Wintermantel, E.; Leong, K.W. Biomedical applications of polymer-composite materials: A review. Compos. Sci. Technol. 2001, 61, 1189-1224. 
83. Shastri, V.P. Non-degradable biocompatible polymers in medicine: past, present and future. Curr. Pharm. Biotechnol. 2003, 4, 331-337.

84. Chen, H.; Yuan, L.; Song, W.; Wu, Z.; Li, D. Biocompatible polymer materials: Role of protein-surface interactions. Prog. Polym. Sci. 2008, 33, 1059-1087.

85. Tanaka, M.; Sato, K.; Kitakami, E.; Kobayashi, S.; Hoshiba, T.; Fukushima, K. Design of biocompatible and biodegradable polymers based on intermediate water concept. Polymer J. 2015, 47, 114-121.

86. Lanza, R.P.; Hayes, J.L.; Chick, W.L. Encapsulated cell technology. Nature Biotechnol. 1996, 14, 1107-1111.

87. Shukla, S.C.; Singh, A.; Pandey, A.K.; Mishra, A. Review on production and medical applications of \&-polylysine. Biochem. Eng. J. 2012, 65, 70-81.

88. Agrawal, C.M.; Ray, R.B. Biodegradable polymeric scaffolds for musculoskeletal tissue engineering. J. Biomed. Mater. Res. 2001, 55, 141-150.

89. Kweon, H.; Yoo, M.; Park, I.; Kim, T.; Lee, H.; Lee, S.; Oh, J.; Akaike, T.; Cho, C. A novel degradable polycaprolactone network for tissue engineering. Biomaterials 2003, 24, 801-808.

90. Wang, Y.C.; Zhang, P.H. Electrospun absorbable polycaprolactone (PCL) scaffolds for medical applications. Adv. Mater. Res. 2014, 906, 221-225.

91. Sartori, S.; Chiono, V.; Tonda-Turo, C.; Mattu, C.; Gianluca, C. Biomimetic polyurethanes in nano and regenerative medicine. J. Mater. Chem. B 2014, 2, 5128-5144.

92. Temenoff, J.S.; Mikos, A.G. Injectable biodegradable materials for orthopedic tissue engineering. Biomaterials 2000, 21, 2405-2412.

93. Behravesh, E.; Yasko, A.W.; Engel, P.S.; Mikos, A.G. Synthetic biodegradable polymers for orthopaedic applications. Clin. Orthop. Rel. Res. 1999, 367, S118-S125.

94. Lewandrowski, K.U.; Gresser, J.D.; Wise, D.L.; White, R.L.; Trantolo, D.J. Osteoconductivity of an injectable and bioresorbable poly(propyleneglycol-co-fumaric acid) bone cement. Biomaterials 2000, 21, 293-298.

95. Lee, K.W.; Wang, S.; Fox, B.C.; Ritman, E.L.; Yaszemski, M.J.; Lu, L. Poly(propylene fumarate) bone tissue engineering scaffold fabrication using stereolithography: effects of resin formulations and laser parameters. Biomacromolecules 2007, 8, 1077-1084.

96. Xu, J.; Feng, E.; Song, J. Renaissance of aliphatic polycarbonates: New techniques and biomedical applications. J. Appl. Polym. Sci. 2014, 131, 39822:1-39822:16.

97. Boland, E.D.; Coleman, B.D.; Barnes, C.P.; Simpson, D.G.; Wnek, G.E.; Bowlin, G.L. Electrospinning polydioxanone for biomedical applications. Acta Biomater. 2005, 1, 115-123.

98. Gilbert, J.L. Acrylics in Biomedical Engineering. In Encyclopedia of Materials: Science and Technology; Elsevier: Amsterdam, The Netherlands, 2001; pp. 11-18.

99. Frazer, R.Q.; Byron, R.T.; Osborne, P.B.; West, K.P. PMMA: An essential material in medicine and dentistry. J. Long-Term Eff. Med. Implants 2005, 15, 629-639.

100. Li, Y.W.; Leong, J.C.Y.; Lu, W.W.; Luk, K.D.K.; Cheung, K.M.C.; Chiu, K.Y.; Chow, S.P. A novel injectable bioactive bone cement for spinal surgery: A developmental and preclinical study. J. Biomed. Mater. Res. 2000, 52, 164-170.

101. Mckellop, H.; Shen, F.; Lu, B.; Campbell, P.; Salovey, R. Development of an extremely wear resistant UHMW polyethylene for total hip replacements. J. Orthop. Res. 1999, 17, 157-167. 
102. Kurtz, S.M.; Muratoglu, O.K.; Evans, M.; Edidin, A.A. Advances in the processing, sterilization and crosslinking of ultra-high molecular weight polyethylene for total joint arthroplasty. Biomaterials 1999, 20, 1659-1688.

103. Laurencin, C.T.; Ambrosio, M.A.; Borden, M.D.; Cooper, J.A., Jr. Tissue engineering: Orthopedic applications. Ann. Rev. Biomed. Eng. 1999, 1, 19-46.

104. Meijer, G.J.; Cune, M.S.; van Dooren, M.; de Putter, C.; van Blitterswijk, C.A. A comparative study of flexible (Polyactive ${ }^{\mathrm{TM}}$ ) versus rigid (hydroxylapatite) permucosal dental implants. I. Clinical aspects. J. Oral Rehabil. 1997, 24, 85-92.

105. Meijer, G.J.; Dalmeijer, R.A.; de Putter, C.; van Blitterswijk, C.A. A comparative study of flexible (Polyactive ${ }^{\mathrm{TM}}$ ) versus rigid (hydroxylapatite) permucosal dental implants. II. Histological aspects. J. Oral Rehabil. 1997, 24, 93-101.

106. Waris, E.; Ashammakhi, N.; Lehtimäki, M.; Tulamo, R.M.; Törmälä, P.; Kellomäki, M.; Konttinen, Y.T. Long-term bone tissue reaction to polyethylene oxide/polybutylene terephthalate copolymer (Polyactive ${ }^{\circledR}$ ) in metacarpophalangeal joint reconstruction. Biomaterials 2008, 29, 2509-2515.

107. Svensson, A.; Nicklasson, E.; Harrah, T.; Panilaitis, B.; Kaplan, D.L.; Brittberg, M.; Gatenholm, $\mathrm{P}$. Bacterial cellulose as a potential scaffold for tissue engineering of cartilage. Biomaterials $\mathbf{2 0 0 5}$, 26, 419-431.

108. Rampinelli, G.; di Landro, L.; Fujii, T. Characterization of biomaterials based on microfibrillated cellulose with different modifications. J. Reinf. Plast. Compos. 2010, 29, 1793-1803.

109. Granja, P.L.; Barbosa, M.A.; Pouysége, L.; de Jéso, B.; Rouais, F.; Baquuey, C. Cellulose phosphates as biomaterials. Mineralization of chemically modified regenerated cellulose hydrogels. J. Mater. Sci. 2001, 36, 2163-2172.

110. Granja, P.L.; Jéso, B.D.; Bareille, R.; Rouais, F.; Baquey, C.; Barbosa, M.A. Cellulose phosphates as biomaterials. In vitro biocompatibility studies. React. Funct. Polym. 2006, 66, 728-739.

111. Thomas, V.; Dean, D.R.; Vohra, Y.K. Nanostructured biomaterials for regenerative medicine. Curr. Nanosci. 2006, 2, 155-177.

112. Dee, K.C.; Bizios, R. Mini-review: Proactive biomaterials and bone tissue engineering. Biotechnol. Bioeng. 1996, 50, 438-442.

113. Ashammakhi, N.; Rokkanen, P. Absorbable polyglycolide devices in trauma and bone surgery. Biomaterials 1997, 18, 3-9.

114. Boyan, B.; Lohmann, C.; Somers, A.; Neiderauer, G.; Wozney, J.; Dean, D.; Carnes, D.; Schwartz, Z. Potential of porous poly-D,L-lactide-co-glycolide particles as a carrier for recombinant human bone morphogenetic protein-2 during osteoinduction in vivo. J. Biomed. Mater. Res. 1999, 46, 51-59.

115. Hollinger, J.O.; Leong, K. Poly( $\alpha$-hydroxyacids): Carriers for bone morphogenetic proteins. Biomaterials 1996, 17, 187-194.

116. Griffith, L.G. Polymeric biomaterials. Acta Mater. 2000, 48, 263-277.

117. Peter, S.J.; Miller, M.J.; Yasko, A.W.; Yaszemski, M.J.; Mikos, A.G. Polymer concepts in tissue engineering. J. Biomed. Mater. Res. 1998, 43, 422-427.

118. Ishuang, S.L.; Payne, R.G.; Yaszemski, M.J.; Aufdemorte, T.B.; Bizios, R.; Mikos, A.G. Osteoblast migration on poly( $\alpha$-hydroxy esters). Biotechnol. Bioeng. 1996, 50, 443-451. 
119. Shikinami, Y.; Okuno, M. Bioresorbable devices made of forged composites of hydroxyapatite (HA) particles and poly-L-lactide (PLLA): Part I. Basic characteristics. Biomaterials 1999, 20, 859-877.

120. Khor, E.; Lim, L.Y. Implantable applications of chitin and chitosan. Biomaterials 2003, 24, 2339-2349.

121. Di Martino, A.; Sittinger, M.; Risbud, M.V. Chitosan: A versatile biopolymer for orthopaedic tissue-engineering. Biomaterials 2005, 26, 5983-5990.

122. Piskin, E.; Bölgen, N.; Egri, S.; Isoglu, I.A. Electrospun matrices made of poly( $\alpha$-hydroxy acids) for medical use. Nanomedicine 2007, 2, 441-457.

123. Rezwana, K.; Chena, Q.Z.; Blakera, J.J.; Boccaccini, A.R. Biodegradable and bioactive porous polymer/inorganic composite scaffolds for bone tissue engineering. Biomaterials 2006, 27 , 3413-3431.

124. Seal, B.L.; Otero, T.C.; Panitch, A. Polymeric biomaterials for tissue and organ regeneration. Mater. Sci. Eng. R 2001, 34, 147-230.

125. Mano, J.F.; Sousa, R.A.; Boesel, L.F.; Neves, N.M.; Reis, R.L. Bioinert, biodegradable and injectable polymeric matrix composites for hard tissue replacement: state of the art and recent developments. Compos. Sci. Technol. 2004, 64, 789-817.

126. Middleton, J.; Tipton, A. Synthetic biodegradable polymers as orthopedic devices. Biomaterials 2000, 21, 2335-2346.

127. Coombes, A.G.; Meikle, M.C. Resorbable synthetic polymers as replacements for bone graft. Clin. Mater. 2004, 17, 35-67.

128. De las Heras Alarcón, C.; Pennadam, S.; Alexander, C. Stimuli responsive polymers for biomedical applications. Chem. Soc. Rev. 2005, 34, 276-285.

129. Kohane, D.S.; Langer, R. Polymeric biomaterials in tissue engineering. Pediatric Res. 2008, 63, 487-491.

130. Okada, M. Chemical syntheses of biodegradable polymers. Prog. Polym. Sci. 2002, 27, 87-133.

131. Jordan, J.; Jacob, K.I.; Tannenbaum, R.; Sharaf, M.A.; Jasiuk, I. Experimental trends in polymer nanocomposites - A review. Mater. Sci. Eng. A 2005, 393, 1-11.

132. Liu, X.; Chu, P.K.; Ding, C. Surface modification of titanium, titanium alloys, and related materials for biomedical applications. Mater. Sci. Eng. R 2004, 47, 49-121.

133. Hanawa, T. Biofunctionalization of titanium for dental implant. Jpn. Dent. Sci. Rev. 2010, 46, 93-101.

134. Frosch, K.H.; Stürmer, K.M. Metallic biomaterials in skeletal repair. Eur. J. Trauma 2006, 32, 149-159.

135. Nasab, M.B.; Hassan, M.R.; Sahari, B.B. Metallic biomaterials of knee and hip-A review. Trends Biomater. Artif. Organ. 2010, 24, 69-82.

136. Chen, Q.; Thouas, G.A. Metallic implant biomaterials. Mater. Sci. Eng. R 2015, 87, 1-57.

137. Ryan, G.; Pandit, A.; Apatsidis, D.P. Fabrication methods of porous metals for use in orthopaedic applications. Biomaterials 2006, 27, 2651-2670.

138. Levine, B. A new era in porous metals: applications in orthopaedics. Adv. Eng. Mater. 2008, 10, 788-792. 
139. Chen, C.; Zhang, M. Fabrication methods of porous tantalum metal implants for use as biomaterials. Adv. Mater. Res. 2012, 476-478, 2063-2066.

140. Ma, P.X. Biomimetic materials for tissue engineering. Adv. Drug Deliver. Rev. 2008, 60, 184-198.

141. Kathuria, Y.P. The potential of biocompatible metallic stents and preventing restenosis. Mater. Sci. Eng. A 2006, 417, 40-48.

142. Purnama, A.; Hermawan, H.; Couet, J.; Mantovani, D. Assessing the biocompatibility of degradable metallic materials: State-of-the-art and focus on the potential of genetic regulation. Acta Biomater. 2010, 6, 1800-1807.

143. Walker, J.; Shadanbaz, S.; Woodfield, T.B.F.; Staiger, M.P.; Dias, G.J. Magnesium biomaterials for orthopedic application: a review from a biological perspective. J. Biomed. Mater. Res. B Appl. Biomater. 2014, 102, 1316-1331.

144. Knowles, J.C. Phosphate based glasses for biomedical applications. J. Mater. Chem. 2003, 13, 2395-2401.

145. Tilocca, A. Current challenges in atomistic simulations of glasses for biomedical applications. Phys. Chem. Chem. Phys. 2014, 16, 3874-3880.

146. Kasuga, T. Development of phosphate glass-ceramics for biomedical applications. J. Ceram. Soc. Jpn. 2007, 115, 455-459.

147. Abdelghany, A.M.; El Batal, F.H.; El Batal, H.A. Zinc containing borate glasses and glassceramics: Search for biomedical applications. Process. Appl. Ceram. 2014, 8, 185-193.

148. Hench, L.L. Bioceramics. J. Am. Ceram. Soc. 1998, 81, 1705-1728.

149. Hench, L.L. The story of Bioglass ${ }^{\circledR}$. J. Mater. Sci. Mater. Med. 2006, 17, 967-978.

150. Jones, J.R. Review of bioactive glass: From Hench to hybrids. Acta Biomater. 2013, 9, 4457-4486.

151. Weizhong, Y.; Dali, Z.; Guangfu, Y. Research and development of A-W bioactive glass ceramic. J. Biomed. Eng. 2003, 20, 541-545.

152. Li, G.; Zhou, D.; Xue, M.; Yang, W.; Long, Q.; Cao, B.; Feng, D. Study on the surface bioactivity of novel magnetic A-W glass ceramic in vitro. Appl. Surf. Sci. 2008, 255, 559-561.

153. Höland, W.; Schweiger, M.; Watzke, R.; Peschke, A.; Kappert, H. Ceramics as biomaterials for dental restoration. Expert Rev. Med. Dev. 2008, 5, 729-745.

154. Li, R.W.K.; Chow, T.W.; Matinlinna, J.P. Ceramic dental biomaterials and CAD/CAM technology: State of the art. J. Prosthodont. Res. 2014, 58, 208-216.

155. Ramesh, T.R.; Gangaiah, M.; Harish, P.V.; Krishnakumar, U.; Nandakishore, B. Zirconia ceramics as a dental biomaterial-An over view. Trends Biomater. Artif. Organs 2012, 26, 154-160.

156. Wang, C.H.; Wang, M.C.; Du, J.K.; Sie, Y.Y.; Hsi, C.S.; Lee, H.E. Phase transformation and nanocrystallite growth behavior of $2 \mathrm{~mol} \%$ yttria-partially stabilized zirconia (2Y-PSZ) powders. Ceram. Int. 2013, 39, 5165-5174.

157. Garvie, R.C. A personal history of the development of transformation toughened PSZ ceramics. J. Aust. Ceram. Soc. 2014, 50, 15-22.

158. Benson, J. Elemental carbon as a biomaterial. J. Biomed. Mater. Res. 1972, 5, 41-47. 
159. Olborska, A.; Swider, M.; Wolowiec, R.; Niedzielski, P.; Rylski, A.; Mitura, S. Amorphous carbon - biomaterial for implant coatings. Diamond Relat. Mater. 1994, 3, 899-901.

160. Saito, N.; Usui, Y.; Aoki, K.; Narita, N.; Shimizu, M.; Hara, K.; Ogiwara, N.; Nakamura, K.; Ishigaki, N.; Kato, H.; et al. Carbon nanotubes: biomaterial applications. Chem. Soc. Rev. 2009, 38, 1897-1903.

161. Saito, N.; Haniu, H.; Usui, Y.; Aoki, K.; Hara, K.; Takanashi, S.; Shimizu, M.; Narita, N.; Okamoto, M.; Kobayashi, S.; et al. Safe clinical use of carbon nanotubes as innovative biomaterials. Chem. Rev. 2014, 114, 6040-6079.

162. Chlopek, J.; Czajkowska, B.; Szaraniec, B.; Frackowiak, E.; Szostak, K.; Beguin, F. In vitro studies of carbon nanotubes biocompatibility. Carbon 2006, 44, 1106-1111.

163. Saito, N.; Usui, Y.; Aoki, K.; Narita, N.; Shimizu, M.; Ogiwara, N.; Nakamura, K.; Ishigaki, N.; Kato, H.; Taruta, S. Carbon nanotubes for biomaterials in contact with bone. Curr. Med. Chem. 2008, 15, 523-527.

164. Banerjee, S.; Kahn, M.G.C.; Wong, S.S. Rational chemical strategies for carbon nanotube functionalization. Chem. Eur. J. 2003, 9, 1898-1908.

165. Beuvelot, J.; Bergeret, C.; Mallet, R.; Fernandez, V.; Cousseau, J.; Baslé, M.F.; Chappard, D. In vitro calcification of chemically functionalized carbon nanotubes. Acta Biomater. 2010, 6, 4110-4117.

166. Xiao, Y.; Gong, T.; Zhou, S. The functionalization of multi-walled carbon nanotubes by in situ deposition of hydroxyapatite. Biomaterials 2010, 31, 5182-5190.

167. Converse, G.L.; Yue, W.; Roeder, R.K. Processing and tensile properties of hydroxyapatitewhisker-reinforced polyetheretherketone. Biomaterials 2007, 28, 927-935.

168. Converse, G.L.; Roeder, R.K. Tensile properties of hydroxyapatite whisker reinforced polyetheretherketone. Mater. Res. Soc. Symp. Proc. 2005, 898, 44-49.

169. Choi, W.Y.; Kim, H.E.; Kim, M.J.; Kim, U.C.; Kim, J.H.; Koh, Y.H. Production and characterization of calcium phosphate $(\mathrm{CaP})$ whisker-reinforced poly( $\varepsilon$-caprolactone) composites as bone regenerative. Mater. Sci. Eng. C 2010, 30, 1280-1284.

170. Zhang, H.; Darvell, B.W. Failure and behavior in water of hydroxyapatite whisker-reinforced bis-GMA-based resin composites. J. Mech. Behav. Biomed. Mater. 2012, 10, 39-47.

171. Liu, F.; Wang, R.; Cheng, Y.; Jiang, X.; Zhang, Q.; Zhu, M. Polymer grafted hydroxyapatite whisker as a filler for dental composite resin with enhanced physical and mechanical properties. Mater. Sci. Eng. C 2013, 33, 4994-5000.

172. Liu, F.W.; Bao, S.; Jin, Y.; Jiang, X.Z.; Zhu, M.F. Novel bionic dental resin composite reinforced by hydroxyapatite whisker. Mater. Res. Innov. 2014, 18, S4854-S4858.

173. Nouri-Felekori, M.; Mesgar, A.S.M.; Mohammadi, Z. Development of composite scaffolds in the system of gelatin - Calcium phosphate whiskers/fibrous spherulites for bone tissue engineering. Ceram. Int. 2015, 41, 6013-6019.

174. Watanabe, T.; Ban, S.; Ito, T.; Tsuruta, S.; Kawai, T.; Nakamura, H. Biocompatibility of composite membrane consisting of oriented needle-like apatite and biodegradable copolymer with soft and hard tissues in rats. Dental Mater. J. 2004, 23, 609-612. 
175. Li, H.; Chen, Y.; Xie, Y. Photo-crosslinking polymerization to prepare polyanhydride/needlelike hydroxyapatite biodegradable nanocomposite for orthopedic application. Mater. Lett. 2003, 57, 2848-2854.

176. Nejati, E.; Firouzdor, V.; Eslaminejad, M.B.; Bagheri, F. Needle-like nano hydroxyapatite/ poly(L-lactide acid) composite scaffold for bone tissue engineering application. Mater. Sci. Eng. C 2009, 29, 942-949.

177. Sun, S.P.; Wei, M.; Olson, J.R.; Shaw, M.T. A modified pultrusion process for preparing composites reinforced with continuous fibers and aligned hydroxyapatite nano needles. Polym. Compos. 2015, 36, 931-938.

178. Kasuga, T.; Ota, Y.; Nogami, M.; Abe, Y. Preparation and mechanical properties of polylactic acid composites containing hydroxyapatite fibers. Biomaterials 2000, 22, 19-23.

179. Smith, L. Ceramic-plastic material as a bone substitute. Arch. Surg. 1963, 87, 653-661.

180. Bonfield, W.; Grynpas, M.D.; Tully, A.E.; Bowman, J.; Abram, J. Hydroxyapatite reinforced polyethylene-A mechanically compatible implant material for bone replacement. Biomaterials 1981, 2, 185-189.

181. Bonfield, W.; Bowman, J.; Grynpas, M.D. Composite material for use in orthopaedics. UK Patent 8032647, 1981.

182. Bonfield, W. Composites for bone replacement. J. Biomed. Eng. 1988, 10, 522-526.

183. Guild, F.J.; Bonfield, W. Predictive character of hydroxyapatite-polyethelene HAPEX ${ }^{\mathrm{TM}}$ composite. Biomaterials 1993, 14, 985-993.

184. Huang, J.; di Silvio, L.; Wang, M.; Tanner, K.E.; Bonfield, W. In vitro mechanical and biological assessment of hydroxyapatite-reinforced polyethylene composite. J. Mater. Sci. Mater. Med. 1997, 8, 775-779.

185. Wang, M.; Joseph, R.; Bonfield, W. Hydroxyapatite-polyethylene composites for bone substitution: effect of ceramic particle size and morphology. Biomaterials 1998, 19, 2357-2366.

186. Ladizesky, N.H.; Ward, I.M.; Bonfield, W. Hydroxyapatite/high-performance polyethylene fiber composites for high load bearing bone replacement materials. J. Appl. Polym. Sci. 1997, 65, 1865-1882.

187. Nazhat, S.N.; Joseph, R.; Wang, M.; Smith, R.; Tanner, K.E.; Bonfield, W. Dynamic mechanical characterisation of hydroxyapatite reinforced polyethylene: effect of particle size. J. Mater. Sci. Mater. Med. 2000, 11, 621-628.

188. Guild, F.J.; Bonfield, W. Predictive modelling of the mechanical properties and failure processes of hydroxyapatite-polyethylene (HAPEX'M) composite. J. Mater. Sci. Mater. Med. 1998, 9, 497-502.

189. Wang M.; Ladizesky NH.; Tanner, K.E.; Ward IM.; Bonfield, W. Hydrostatically extruded HAPEX ${ }^{\mathrm{TM}}$. J. Mater. Sci. 2000, 35, 1023-1030.

190. That PT.; Tanner, K.E.; Bonfield, W. Fatigue characterization of a hydroxyapatite-reinforced polyethylene composite. I. Uniaxial fatigue. J. Biomed. Mater. Res. 2000, 51, 453-460.

191. That PT.; Tanner, K.E.; Bonfield, W. Fatigue characterization of a hydroxyapatite-reinforced polyethylene composite. II. Biaxial fatigue. J. Biomed. Mater. Res. 2000, 51, 461-468.

192. Bonner M.; Saunders LS.; Ward IM.; Davies GW.; Wang M.; Tanner, K.E.; Bonfield, W. Anisotropic mechanical properties of oriented HAPEX ${ }^{\mathrm{TM}}$. J. Mater. Sci. 2002, 37, 325-334. 
193. Di Silvio, L.; Dalby, M.J.; Bonfield, W. Osteoblast behaviour on HA/PE composite surfaces with different HA volumes. Biomaterials 2002, 23, 101-107.

194. Dalby, M.J.; Kayser, M.V.; Bonfield, W.; di Silvio, L. Initial attachment of osteoblasts to an optimised HAPEX ${ }^{\mathrm{TM}}$ topography. Biomaterials 2002, 23, 681-690.

195. Zhang, Y.; Tanner, K.E.; Gurav, N.; di Silvio, L. In vitro osteoblastic response to 30 vol\% hydroxyapatite-polyethylene composite. J. Biomed. Mater. Res. A 2007, 81, 409-417.

196. Rea, S.M.; Brooks, R.A.; Schneider, A.; Best, S.M.; Bonfield, W. Osteoblast-like cell response to bioactive composites-surface-topography and composition effects. J. Biomed. Mater. Res. B Appl. Biomater. 2004, 70, 250-261.

197. Salernitano, E.; Migliaresi, C. Composite materials for biomedical applications: A review. $J$. Appl. Biomater. Biomech. 2003, 1, 3-18.

198. Pandey, A.; Jan, E.; Aswath, P.B. Physical and mechanical behavior of hot rolled HDPE/HA composites. J. Mater. Sci. 2006, 41, 3369-3376.

199. Bonner M.; Ward IM.; McGregor W.; Tanner, K.E.; Bonfield, W. Hydroxyapatite/polypropylene composite: a novel bone substitute material. J. Mater. Sci. Lett. 2001, 20, 2049-2052.

200. Suppakarn, N.; Sanmaung, S.; Ruksakulpiwa, Y.; Sutapun, W. Effect of surface modification on properties of natural hydroxyapatite/polypropylene composites. Key Eng. Mater. 2008, 361-363, 511-514.

201. Younesi, M.; Bahrololoom, M.E. Formulating the effects of applied temperature and pressure of hot pressing process on the mechanical properties of polypropylene-hydroxyapatite bio-composites by response surface methodology. Mater. Des. 2010, 31, 4621-4630.

202. Younesi, M.; Bahrololoom, M.E. Effect of polypropylene molecular weight, hydroxyapatite particle size, and Ringer's solution on creep and impact behavior of polypropylene-surface treated hydroxyapatite biocomposites. J. Compos. Mater. 2011, 45, 513-523.

203. Sousa, R.A.; Reis, R.L.; Cunha, A.M.; Bevis, M.J. Processing and properties of bone-analogue biodegradable and bioinert polymeric composites. Compos. Sci. Technol. 2003, 63, 389-402.

204. Wang, M.; Deb, S.; Bonfield, W. Chemically coupled hydroxyapatite-polyethylene composites: processing and characterisation. Mater. Lett. 2000, 44, 119-124.

205. Wang, M.; Bonfield, W. Chemically coupled hydroxyapatite-polyethylene composites: structure and properties. Biomaterials 2001, 22, 1311-1320.

206. Homaeigohar, S.S.; Shokrgozar, M.A.; Khavandi, A.; Sadi, A.Y. In vitro biological evaluation of $\beta$-TCP/HDPE—A novel orthopedic composite: A survey using human osteoblast and fibroblast bone cells. J. Biomed. Mater. Res. A 2008, 84A, 491-499.

207. Sadi, A.Y.; Homaeigohar, S.SH.; Khavandi, A.R.; Javadpour, J. The effect of partially stabilized zirconia on the mechanical properties of the hydroxyapatite-polyethylene composites. J. Mater. Sci. Mater. Med. 2004, 15, 853-858.

208. Nath, S.; Bodhak, S.; Basu, B. HDPE-Al ${ }_{2} \mathrm{O}_{3}-\mathrm{HAp}$ composites for biomedical applications: processing and characterizations. J. Biomed. Mater. Res. B Appl. Biomater. 2009, 88, 1-11.

209. Downes, R.N.; Vardy, S.; Tanner, K.E.; Bonfield, W. Hydroxyapatite-polyethylene composite in orbital surgery. Bioceramics 1991, 4, 46.

210. Dornhoffer, H.L. Hearing results with the dornhoffer ossicular replacement prostheses. Laryngoscope 1998, 108, 531-536. 
211. Swain, R.E.; Wang, M.; Beale, B.; Bonfield, W. HAPEX ${ }^{\mathrm{TM}}$ for otologic applications. Biomed. Eng. Appl. Basis Commun. 1999, 11, 315-320.

212. Zuo, Y.; Li, Y.; Li, J.; Zhang, X.; Liao, H.; Wang, Y.; Yang, W. Novel bio-composite of hydroxyapatite reinforced polyamide and polyethylene: Composition and properties. Mater. Sci. Eng. A 2007, 452-453, 512-517.

213. Unwin, A.P.; Ward, I.M.; Ukleja, P.; Weng, J. The role of pressure annealing in improving the stiffness of polyethylene/hydroxyapatite composites. J. Mater. Sci. 2001, 36, 3165-3177.

214. Fang, L.M.; Leng, Y.; Gao, P. Processing and mechanical properties of HA/UHMWPE nanocomposites. Biomaterials 2006, 27, 3701-3707.

215. Fang, L.M.; Gao, P.; Leng, Y. High strength and bioactive hydroxyapatite nano-particles reinforced ultrahigh molecular weight polyethylene. Composites B 2007, 38, 345-351.

216. Fang, L.M.; Leng, Y.; Gao, P. Processing of hydroxyapatite reinforced ultrahigh molecular weight polyethylene for biomedical applications. Biomaterials 2005, 26, 3471-3478.

217. Selvin, T.P.; Seno, J.; Murukan, B.; Santhosh, A.A.; Sabu, T.; Weimin, Y.; Sri, B. Poly(ethyleneco-vinyl acetate)/calcium phosphate nanocomposites: thermo mechanical and gas permeability measurements. Polym. Composite 2010, 31, 1011-1019.

218. Reis, R.L.; Cunha, A.M.; Oliveira, M.J.; Campos, A.R.; Bevis, M.J. Relationship between processing and mechanical properties of injection molded high molecular mass polyethylene + hydroxyapatite composites. Mater. Res. Inn. 2001, 4, 263-272.

219. Sousa, R.A.; Reis, R.L.; Cunha, A.M.; Bevis, M.J. Structure development and interfacial interactions in high-density polyethylene/hydroxyapatite (HDPE/HA) composites molded with preferred orientation. J. Appl. Polym. Sci. 2002, 86, 2873-2886.

220. Mirsalehi, S.A.; Khavandi, A.; Mirdamadi, S.; Naimi-Jamal, M.R.; Kalantari, S.M. Nanomechanical and tribological behavior of hydroxyapatite reinforced ultrahigh molecular weight polyethylene nanocomposites for biomedical applications. J. Appl. Polym. Sci. 2015, 132, doi:10.1002/app.42052.

221. Donners, J.J.J.M.; Nolte, R.J.M.; Sommerdijk, N.A.J.M. Dendrimer-based hydroxyapatite composites with remarkable materials properties. Adv. Mater. 2003, 15, 313-316.

222. Schneider, O.D.; Stepuk, A.; Mohn, D.; Luechinger, N.A.; Feldman, K.; Stark, W.J. Lightcurable polymer/calcium phosphate nanocomposite glue for bone defect treatment. Acta Biomater. 2010, 6, 2704-2710.

223. Ignjatovic, N.L.; Plavsic, M.; Miljkovic, M.S.; Zivkovic, L.M.; Uskokovic, D.P. Microstructural characteristics of calcium hydroxyapatite/poly-L-lactide based composites. J. Microsc. 1999, 196, 243-248.

224. Skrtic, D.; Antonucci, J.M.; Eanes, E.D. Amorphous calcium phosphate-based bioactive polymeric composites for mineralized tissue regeneration. J. Res. Natl. Inst. Stand. Technol. 2003, 108, 167-182.

225. Rizzi, S.C.; Heath, D.J.; Coombes, A.G.A.; Bock, N.; Textor, M.; Downes, S. Biodegradable polymer/hydroxyapatite composites: Surface analysis and initial attachment of human osteoblasts. J. Biomed. Mater. Res. 2001, 55, 475-486.

226. Navarro, M.; Planell, J.A. Bioactive composites based on calcium phosphates for bone regeneration. Key Eng. Mater. 2010, 441, 203-233. 
227. Zhang, R.Y.; Ma, P.X. Porous poly(L-lactic acid)/apatite composites created by biomimetic process. J. Biomed. Mater. Res. 1999, 45, 285-293.

228. Liu, Q.; de Wijn, J.R.; van Blitterswijk, C.A. Composite biomaterials with chemical bonding between hydroxyapatite filler particles and PEG/PBT copolymer matrix. J. Biomed. Mater. Res. 1998, 40, 490-497.

229. Cerrai, P.; Guerra, G.D.; Tricoli, M.; Krajewski, A.; Ravaglioli, A.; Martinetti, R.; Dolcini, L. Fini, M.; Scarano, A.; Piattelli, A. Periodontal membranes from composites of hydroxyapatite and bioresorbable block copolymers. J. Mater. Sci. Mater. Med. 1999, 10, 677-682.

230. Roeder, R.K.; Sproul, M.M.; Turner, C.H. Hydroxyapatite whiskers provide improved mechanical properties in reinforced polymer composites. J. Biomed. Mater. Res. A 2003, 67, 801-812.

231. Wagoner Johnson, A.J.; Herschler, B.A. A review of the mechanical behavior of CaP and $\mathrm{CaP} /$ polymer composites for applications in bone replacement and repair. Acta Biomater. 2011, 7, 16-30.

232. Hutmacher, D.W. Scaffolds in tissue engineering bone and cartilage. Biomaterials 2000, 21, 2529-2543.

233. Mathieu, L.M.; Bourban, P.E.; Manson, J.A.E. Processing of homogeneous ceramic/polymer blends for bioresorbable composites. Compos. Sci. Technol. 2006, 66, 1606-1614.

234. Redepenning, J.; Venkataraman, G.; Chen, J.; Stafford, N. Electrochemical preparation of chitosan/hydroxyapatite composite coatings on titanium substrates. J. Biomed. Mater. Res. A 2003, 66, 411-416.

235. Rhee, S.H.; Tanaka, J. Synthesis of a hydroxyapatite/collagen/chondroitin sulfate nanocomposite by a novel precipitation method. J. Am. Ceram. Soc. 2001, 84, 459-461.

236. Pezzotti, G.; Asmus, S.M.F. Fracture behavior of hydroxyapatite/polymer interpenetrating network composites prepared by in situ polymerization process. Mater. Sci. Eng. A 2001, 316, 231-237.

237. Weickmann, H.; Gurr, M.; Meincke, O.; Thomann, R.; Mülhaupt, R. A versatile solvent-free "one-pot" route to polymer nanocomposites and the in situ formation of calcium phosphate/layered silicate hybrid nanoparticles. Adv. Funct. Mater. 2010, 20, 1778-1786.

238. Aryal, S.; Bhattarai, S.R.; Bahadur, K.C.R.; Khil, M.S.; Lee, D.R.; Kim, H.Y. Carbon nanotubes assisted biomimetic synthesis of hydroxyapatite from simulated body fluid. Mater. Sci. Eng. A 2006, 426, 202-207.

239. Kealley, C.; Ben-Nissan, B.; van Riessen, A.; Elcombe, M. Development of carbon nanotube reinforced hydroxyapatite bioceramics. Key Eng. Mater. 2006, 309-311, 597-600.

240. Kealley, C.; Elcombe, M.; van Riessen, A.; Ben-Nissan, B. Development of carbon nanotube reinforced hydroxyapatite bioceramics. Physica B 2006, 385-386, 496-498.

241. Aryal, S.; Bahadur, K.C.R.; Dharmaraj, N.; Kim, K.W.; Kim, H.Y. Synthesis and characterization of hydroxyapatite using carbon nanotubes as a nano-matrix. Scripta Mater. 2006, 54, 131-135.

242. Rautaray, D.; Mandal, S.; Sastry, M. Synthesis of hydroxyapatite crystals using amino acid-capped gold nanoparticles as a scaffold. Langmuir 2005, 21, 5185-5191.

243. Wang, X.J.; Li, Y.; Wei, J.; de Groot, K. Development of biomimetic nano-hydroxyapatite/ poly(hexamethylene adipamide) composites. Biomaterials 2002, 23, 4787-4791. 
244. Wei, J.; Li, Y. Tissue engineering scaffold material of nano-apatite crystals and polyamide composite. Eur. Polym. J. 2004, 40, 509-515.

245. Memoto, R.; Nakamura, S.; Isobe, T.; Senna, M. Direct synthesis of hydroxyapatite-silk fibroin nano-composite sol via a mechano-chemical route. J. Sol Gel Sci. Technol. 2001, 21, 7-12.

246. Yoshida, A.; Miyazaki, T.; Ashizuka, M.; Ishida, E. Bioactivity and mechanical properties of cellulose/carbonate hydroxyapatite composites prepared in situ through mechanochemical reaction. J. Biomater. Appl. 2006, 21, 179-194.

247. Fujiwara, M.; Shiokawa, K.; Morigaki, K.; Tatsu, Y.; Nakahara, Y. Calcium phosphate composite materials including inorganic powders, BSA or duplex DNA prepared by $\mathrm{W} / \mathrm{O} / \mathrm{W}$ interfacial reaction method. Mater. Sci. Eng. C 2008, 28, 280-288.

248. Nagata, F.; Miyajima, T.; Yokogawa, Y. A method to fabricate hydroxyapatite/poly(lactic acid) microspheres intended for biomedical application. J. Eur. Ceram. Soc. 2006, 26, 533-535.

249. Russias, J.; Saiz, E.; Nalla, R.K.; Tomsia, A.P. Microspheres as building blocks for hydroxyapatite/polylactide biodegradable composites. J. Mater. Sci. 2006, 41, 5127-5133.

250. Khan, Y.M.; Cushnie, E.K.; Kelleher, J.K.; Laurencin, C.T. In situ synthesized ceramic-polymer composites for bone tissue engineering: bioactivity and degradation studies. J. Mater. Sci. 2007, $42,4183-4190$.

251. Liu, X.; Okada, M.; Maeda, H.; Fujii, S.; Furuzono, T. Hydroxyapatite/biodegradable poly(L-lactide- $c o$ - $\varepsilon$-caprolactone) composite microparticles as injectable scaffolds by a Pickering emulsion route. Acta Biomater. 2011, 7, 821-828.

252. Hu, Y.; Zou, S.; Chen, W.; Tong, Z.; Wang, C. Mineralization and drug release of hydroxyapatite/poly(L-lactic acid) nanocomposite scaffolds prepared by Pickering emulsion templating. Colloids Surf. B 2014, 122, 559-565.

253. Kim, H.W.; Knowles, J.C.; Kim, H.E. Hydroxyapatite and gelatin composite foams processed via novel freeze-drying and crosslinking for use as temporary hard tissue scaffolds. J. Biomed. Mater. Res. A 2005, 72A, 136-145.

254. Mohandes, F.; Salavati-Niasari, M. Freeze-drying synthesis, characterization and in vitro bioactivity of chitosan/graphene oxide/hydroxyapatite nanocomposite. RSC Adv. 2014, 4, 25993-26001.

255. Sinha, A.; Das, G.; Sharma, B.K.; Roy, R.P.; Pramanick, A.K.; Nayar, S. Poly(vinyl alcohol)hydroxyapatite biomimetic scaffold for tissue regeneration. Mater. Sci. Eng. C 2007, 27, 70-74.

256. Sugawara, A.; Yamane, S.; Akiyoshi, K. Nanogel-templated mineralization: polymer-calcium phosphate hybrid nanomaterials. Macromol. Rapid Commun. 2006, 27, 441-446.

257. Kickelbick, G. Concepts for the incorporation of inorganic building blocks into organic polymers on a nanoscale. Prog. Polym. Sci. 2003, 28, 83-114.

258. Liu, Q.; de Wijn, J.R.; van Blitterswijk, C.A. Nanoapatite/polymer composites: mechanical and physicochemical characteristics. Biomaterials 1997, 18, 1263-1270.

259. Uskokovic, P.S.; Tang, C.Y.; Tsui, C.P.; Ignjatovic, N.; Uskokovic, D.P. Micromechanical properties of a hydroxyapatite/poly-L-lactide biocomposite using nanoindentation and modulus mapping. J. Eur. Ceram. Soc. 2007, 27, 1559-1564.

260. Todo, M.; Kagawa, T. Improvement of fracture energy of HA/PLLA biocomposite material due to press processing. J. Mater. Sci. 2008, 43, 799-801. 
261. Woo, K.M.; Seo, J.; Zhang, R.Y.; Ma, P.X. Suppression of apoptosis by enhanced protein adsorption on polymer/hydroxyapatite composite scaffolds. Biomaterials 2007, 28, 2622-2630.

262. Baji, A.; Wong, S.C.; Srivatsan, T.S.; Njus, G.O.; Mathur, G. Processing methodologies for polycaprolactone-hydroxyapatite composites: A review. Mater. Manuf. Process. 2006, 21, 211-218.

263. Guan, L.; Davies, J.E. Preparation and characterization of a highly macroporous biodegradable composite tissue engineering scaffold. J. Biomed. Mater. Res. A 2004, 71, 480-487.

264. Sun, F.; Zhou, H.; Lee, J. Various preparation methods of highly porous hydroxyapatite/polymer nanoscale biocomposites for bone regeneration. Acta Biomater. 2011, 7, 3813-3828.

265. Kumar, A.; Negi, Y.S.; Choudhary, V.; Bhardwaj, N.K. Microstructural and mechanical properties of porous biocomposite scaffolds based on polyvinyl alcohol, nano-hydroxyapatite and cellulose nanocrystals. Cellulose 2015, 21, 3409-3426.

266. Teng, X.R.; Ren, J.; Gu, S.Y. Preparation and characterization of porous PDLLA/HA composite foams by supercritical carbon dioxide technology. J. Biomed. Mater. Res. B Appl. Biomater. 2007, 81, 185-193.

267. Ren, J.; Zhao, P.; Ren, T.; Gu, S.; Pan, K. Poly (D,L-lactide)/nano-hydroxyapatite composite scaffolds for bone tissue engineering and biocompatibility evaluation. J. Mater. Sci. Mater. Med. 2008, 19, 1075-1082.

268. Wang, M.; Yue, C.Y.; Chua, B. Production and evaluation of hydroxyapatite reinforced polysulfone for tissue replacement. J. Mater. Sci. Mater. Med. 2001, 12, 821-826.

269. Chlopek, J.; Rosol, P.; Morawska-Chochol, A. Durability of polymer-ceramics composite implants determined in creep tests. Compos. Sci. Technol. 2006, 66, 1615-1622.

270. Robinson, P.; Wilson, C.; Mecholsky, J. Processing and mechanical properties of hydroxyapatitepolysulfone laminated composites. J. Eur. Ceram. Soc. 2014, 34, 1387-1396.

271. Xu, F.; Li, Y.; Yao, X.; Liao, H.; Zhang, L. Preparation and in vivo investigation of artificial cornea made of nano-hydroxyapatite/poly (vinyl alcohol) hydrogel composite. J. Mater. Sci. Mater. Med. 2007, 18, 635-640.

272. Xu, F.; Li, Y.; Deng, Y.; Xiong, G. Porous nano-hydroxyapatite/poly(vinyl alcohol) composite hydrogel as artificial cornea fringe: characterization and evaluation in vitro. J. Biomater. Sci. Polym. Edn. 2008, 19, 431-439.

273. Nayar, S.; Pramanick, A.K.; Sharma, B.K.; Das, G.; Kumar, B.R.; Sinha, A. Biomimetically synthesized polymer-hydroxyapatite sheet like nano-composite. J. Mater. Sci. Mater. Med. 2008, 19, 301-304.

274. Poursamar, S.A.; Orang, F.; Bonakdar, S.; Savar, M.K. Preparation and characterisation of poly vinyl alcohol/hydroxyapatite nanocomposite via in situ synthesis: a potential material as bone tissue engineering scaffolds. Int. J. Nanomanuf. 2010, 5, 330-334.

275. Guha, A.; Nayar, S.; Thatoi, H.N. Microwave irradiation enhances kinetics of the biomimetic process of hydroxyapatite nanocomposites. Bioinspir. Biomim. 2010, 5, 024001:1- 024001:5.

276. Pramanik, N.; Biswas, S.K.; Pramanik, P. Synthesis and characterization of hydroxyapatite/ poly(vinyl alcohol phosphate) nanocomposite biomaterials. Int. J. Appl. Ceram. Technol. 2008, $5,20-28$. 
277. Bigi, A.; Boanini, E.; Gazzano, M.; Rubini, K. Structural and morphological modifications of hydroxyapatite-polyaspartate composite crystals induced by heat treatment. Cryst. Res. Technol. 2005, 40, 1094-1098.

278. Bertoni, E.; Bigi, A.; Falini, G.; Panzavolta, S.; Roveri, N. Hydroxyapatite polyacrylic acid nanocrystals. J. Mater. Chem. 1999, 9, 779-782.

279. Qiu, H.J.; Yang, J.; Kodali, P.; Koh, J.; Ameer, G.A. A citric acid-based hydroxyapatite composite for orthopedic implants. Biomaterials 2006, 27, 5845-5854.

280. Greish, Y.E.; Brown, P.W. Chemically formed HAp-Ca poly(vinyl phosphonate) composites. Biomaterials 2001, 22, 807-816.

281. Greish, Y.E.; Brown, P.W. Preparation and characterization of calcium phosphate-poly(vinyl phosphonic acid) composites. J. Mater. Sci. Mater. Med. 2001, 12, 407-411.

282. Greish, Y.E.; Brown, P.W. Formation and properties of hydroxyapatite-calcium poly(vinyl phosphonate) composites. J. Am. Ceram. Soc. 2002, 85, 1738-1744.

283. Sailaja, G.S.; Velayudhan, S.; Sunny, M.C.; Sreenivasan, K.; Varma, H.K.; Ramesh, P. Hydroxyapatite filled chitosan-polyacrylic acid polyelectrolyte complexes. J. Mater. Sci. 2003, $38,3653-3662$.

284. Piticescu, R.M.; Chitanu, G.C.; Albulescu, M.; Giurginca, M.; Popescu, M.L.; Łojkowski, W. Hybrid HAp-maleic anhydride copolymer nanocomposites obtained by in-situ functionalisation. Solid State Phenom. 2005, 106, 47-56.

285. Song, J.; Saiz, E.; Bertozzi, C.R. A new approach to mineralization of biocompatible hydrogel scaffolds: an efficient process toward 3-dimensional bonelike composites. J. Am. Chem. Soc. 2003, 125, 1236-1243.

286. Kutikov, A.B.; Song J. An amphiphilic degradable polymer/hydroxyapatite composite with enhanced handling characteristics promotes osteogenic gene expression in bone marrow stromal cells. Acta Biomater. 2013, 9, 8354-8364.

287. Abu Bakar, M.S.; Cheng, M.H.W.; Tang, S.M.; Yu, S.C.; Liao, K.; Tan, C.T.; Khor, K.A.; Cheang, P. Tensile properties, tension-tension fatigue and biological response of polyetheretherketone-hydroxyapatite composites for load-bearing orthopedic implants. Biomaterials 2003, 24, 2245-2250.

288. Abu Bakar, M.S.; Cheang, P.; Khor, K.A. Mechanical properties of injection molded hydroxyapatite-polyetheretherketone biocomposites. Compos. Sci. Technol. 2003, 63, 421-425.

289. Abu Bakar, M.S.; Cheang, P.; Khor, K.A. Tensile properties and microstructural analysis of spheroidized hydroxyapatite-poly(etheretherketone) biocomposites. Mater. Sci. Eng. A 2003, 345, 55-63.

290. Fan, J.P.; Tsui, C.P.; Tang, C.Y. Modeling of the mechanical behavior of HA/PEEK biocomposite under quasi-static tensile load. Mater. Sci. Eng. A 2004, 382, 341-350.

291. Wang, L.; Weng, L.; Song, S.; Sun, Q. Mechanical properties and microstructure of polyetheretherketone-hydroxyapatite nanocomposite materials. Mater. Lett. 2010, 64, 2201-2204.

292. Li, K.; Yeung, C.Y.; Yeung, K.W.K.; Tjong, S.C. Sintered hydroxyapatite/polyetheretherketone nanocomposites: Mechanical behavior and biocompatibility. Adv. Eng. Mater. 2012, 14, B155-B165. 
293. Wang, L.; He, S.; Wu, X.; Liang, S.; Mu, Z.; Wei, J.; Deng, F.; Deng, Y.; Wei, S. Polyetheretherketone/nano-fluorohydroxyapatite composite with antimicrobial activity and osseointegration properties. Biomaterials 2014, 35, 6758-6775.

294. Gong, X.H.; Tang, C.Y.; Hu, H.C.; Zhou, X.P. Improved mechanical properties of HIPS/hydroxyapatite composites by surface modification of hydroxyapatite via in situ polymerization of styrene. J. Mater. Sci. Mater. Med. 2004, 15, 1141-1146.

295. Fu, G.; Xia, Z.; Jiang, J.; Jing, B.; Zhang, X. Fabrication and characterization of nanocomposites with high-impact polystyrene and hydroxyapatite with well-defined polystyrene via ATRP. $J$. Reinf. Plast. Comp. 2011, 30, 1445-1453.

296. Petricca, S.E.; Marra, K.G.; Kumta, P.N. Chemical synthesis of poly(lactic-co-glycolic acid)/hydroxyapatite composites for orthopaedic applications. Acta Biomater. 2006, 2, 277-286.

297. Kim, S.S.; Ahn, K.M.; Park, M.S.; Lee, J.H.; Choi, C.Y.; Kim, B.S. A poly(lactide-co-glycolide)/ hydroxyapatite composite scaffold with enhanced osteoconductivity. J. Biomed. Mater. Res. A 2007, 80, 206-215.

298. Oliveira, J.; Miyazaki, T.; Lopes, M.; Ohtsuki, C.; Santos, J. Bonelike ${ }^{\circledR} /$ PLGA hybrid materials for bone regeneration: Preparation route and physicochemical characterization. J. Mater. Sci. Mater. Med. 2005, 16, 253-259.

299. Aboudzadeh, N.; Imani, M.; Shokrgozar, M.A.; Khavandi, A.; Javadpour, J.; Shafieyan, Y.; Farokhi, M. Fabrication and characterization of poly(D,L-lactide-co-glycolide)/ hydroxyapatite nanocomposite scaffolds for bone tissue regeneration. J. Biomed. Mater. Res. A 2010, 94, 137-145.

300. Zhou, H.; Lawrence, J.G.; Bhaduri, S.B. Fabrication aspects of PLA-CaP/PLGA-CaP composites for orthopedic applications: a review. Acta Biomater. 2012, 8, 1999-2016.

301. Hoekstra, J.W.M.; Ma, J.; Plachokova, A.S.; Bronkhorst, E.M.; Bohner, M.; Pan, J.; Meijer, G.J.; Jansen, J.A.; van den Beucken, J.J.J.P. The in vivo performance of CaP/PLGA composites with varied PLGA microsphere sizes and inorganic compositions. Acta Biomater. 2013, 9, 7518-7526.

302. Leung, L.H.; Naguib, H.E. Characterizing the viscoelastic behaviour of poly(lactide-co-glycolide acid)-hydroxyapatite foams. J. Cell. Plast. 2013, 49, 497-505.

303. Takeoka, Y.; Hayashi, M.; Sugiyama, N.; Yoshizawa-Fujita, M.; Aizawa, M.; Rikukawa, M. In situ preparation of poly(1-lactic acid-co-glycolic acid)/hydroxyapatite composites as artificial bone materials. Polym. J. 2015, 47, 164-170.

304. Fisher, P.D.; Venugopal, G.; Milbrandt, T.A.; Hilt, J.Z.; Puleo, D.A. Hydroxyapatite-reinforced in situ forming PLGA systems for intraosseous injection. J. Biomed. Mater. Res. A 2015, 103, 2365-2373.

305. Athanasiou, K.A.; Schmitz, J.P.; Agrawal, C.M. The effects of porosity on in vitro degradation of polylactic acid- polyglycolic acid implants used in repair of articular cartilage. Tissue Eng. 1998, 4, 53-63.

306. Verheyen, C.C.P.M.; Klein, C.P.A.T.; de Blieck-Hogervorst, J.M.A.; Wolke, J.G.C.; de Wijin, J.R.; van Blitterswijk, C.A.; de Groot, K. Evaluation of hydroxylapatite poly(L-lactide) composites: physico-chemical properties. J. Mater. Sci. Mater. Med. 1993, 4, 58-65.

307. Agrawal, C.M.; Athanasiou, K.A. Technique to control pH in vicinity of biodegrading PLA-PGA implants. J. Biomed. Mater. Res. Appl. Biomater. 1997, 38, 105-114. 
308. Li, H.; Chang, J. pH-compensation effect of bioactive inorganic fillers on the degradation of PLGA. Compos. Sci. Technol. 2005, 65, 2226-2232.

309. Peter, S.J.; Miller, S.T.; Zhu, G.; Yasko, A.W.; Mikos, A.G. In vivo degradation of a poly(propylene fumarate)/ $\beta$-tricalcium phosphate injectable composite scaffold. J. Biomed. Mater. Res. 1998, 41, 1-7.

310. Ara, M.; Watanabe, M.; Imai, Y. Effect of blending calcium compounds on hydrolitic degradation of poly(D,L-lactic acid-co-glycolic acid). Biomaterials 2002, 23, 2479-2483.

311. Linhart, W.; Peters, F.; Lehmann, W.; Schwarz, K.; Schilling, A.; Amling, M.; Rueger, J.M.; Epple, M. Biologically and chemically optimized composites of carbonated apatite and polyglycolide as bone substitution materials. J. Biomed. Mater. Res. 2001, 54, 162-171.

312. Schiller, C.; Epple, M. Carbonated apatites can be used as pH-stabilizing filler for biodegradable polyesters. Biomaterials 2003, 24, 2037-2043.

313. Schiller, C.; Rasche, C.; Wehmöller, M.; Beckmann, F.; Eufinger, H.; Epple, M.; Weihe, S. Geometrically structured implants for cranial reconstruction made of biodegradable polyesters and calcium phosphate/calcium carbonate. Biomaterials 2004, 25, 1239-1247.

314. Shikinami, Y.; Okuno, M. Bioresorbable devices made of forged composites of hydroxyapatite (HA) particles and poly L-lactide (PLLA). Part II: Practical properties of miniscrews and miniplates. Biomaterials 2001, 22, 3197-3211.

315. Russias, J.; Saiz, E.; Nalla, R.K.; Gryn, K.; Ritchie, R.O.; Tomsia, A.P. Fabrication and mechanical properties of PLA/HA composites: A study of in vitro degradation. Mater. Sci. Eng. C 2006, 26, 1289-1295.

316. Akagi, H.; Iwata, M.; Ichinohe, T.; Amimoto, H.; Hayashi, Y.; Kannno, N.; Ochi, H.; Fujita, Y.; Harada, Y.; Tagawa, M.; et al. Hydroxyapatite/poly-L-lactide acid screws have better biocompatibility and femoral burr hole closure than does poly-L-lactide acid alone. J. Biomater. Appl. 2014, 28, 954-962.

317. Kim, H.W.; Lee, H.H.; Knowles, J.C. Electrospinning biomedical nanocomposite fibers of hydroxyapaite/poly(lactic acid) for bone regeneration. J. Biomed. Mater. Res. A 2006, 79, 643-649.

318. Gross, K.A.; Rodríguez-Lorenzo, L.M. Biodegradable composite scaffolds with an interconnected spherical network for bone tissue engineering. Biomaterials 2004, 25, 4955-4962.

319. Zhang, H.; Chen, Z. Fabrication and characterization of electrospun PLGA/MWNTs/hydroxyapatite biocomposite scaffolds for bone tissue engineering. J. Bioact. Compat. Polym. 2010, 25, 241-259.

320. Durucan, C.; Brown, P.W. Low temperature formation of calcium-deficient hydroxyapatitePLA/PLGA composites. J. Biomed. Mater. Res. 2000, 51, 717-725.

321. Durucan, C.; Brown, P.W. Calcium-deficient hydroxyapatite-PLGA composites: mechanical and microstructural investigation. J. Biomed. Mater. Res. 2000, 51, 726-734.

322. Durucan, C.; Brown, P.W. Biodegradable hydroxyapatite-polymer composites. Adv. Eng. Mater. 2001, 3, 227-231.

323. Nazhat, S.N.; Kellomäki, M.; Törmälä, P.; Tanner, K.E.; Bonfield, W. Dynamic mechanical characterization of biodegradable composites of hydroxyapatite and polylactides. J. Biomed. Mater. Res. 2001, 58, 335-343. 
324. Ignjatovic, N.; Suljovrujic, E.; Biudinski-Simendic, J.; Krakovsky, I.; Uskokovic, D. Evaluation of hot-presses hydroxyapatite/poly-L-lactide composite biomaterial characteristics. J. Biomed. Mater. Res. B Appl. Biomater. 2004, 71, 284-294.

325. Wang, X.; Lou, T.; Yang, J.; Yang, Z.; He, K. Preparation of PLLA/HAP/ $\beta-T C P$ composite scaffold for bone tissue engineering. Appl. Mech. Mater. 2014, 513-517, 143-146.

326. Hasegawa, S.; Tamura, J.; Neo, M.; Goto, K.; Shikinami, Y.; Saito, M.; Kita, M.; Nakamura, T. In vivo evaluation of a porous hydroxyapatite/poly-D,L-lactide composite for use as a bone substitute. J. Biomed. Mater. Res. A 2005, 75, 567-579.

327. Hasegawa, S.; Neo, M.; Tamura, J.; Fujibayashi, S.; Takemoto, M.; Shikinami, Y.; Okazaki, K.; Nakamura, T. In vivo evaluation of a porous hydroxyapatite/poly-D,L-lactide composite for bone tissue engineering. J. Biomed. Mater. Res. A 2007, 81A, 930-938.

328. Kim, S.S.; Park, M.S.; Jeon, Q.; Choi, C.Y.; Kim, B.S. Poly(lactide-co-glycolide)/hydroxyapatite composite scaffolds for bone tissue engineering. Biomaterials 2006, 27, 1399-1409.

329. Reis, R.L.; Cunha, A.M. New degradable load-bearing biomaterials composed of reinforced starch based blends. J. Appl. Med. Polym. 2000, 4, 1-5.

330. Sousa, R.A.; Mano, J.F.; Reis, R.L.; Cunha, A.M.; Bevis, M.J. Mechanical performance of starch based bioactive composites moulded with preferred orientation for potential medical applications. Polym. Eng. Sci. 2002, 42, 1032-1045.

331. Marques, A.P.; Reis, R.L. Hydroxyapatite reinforcement of different starch-based polymers affects osteoblast-like cells adhesion/spreading and proliferation. Mater. Sci. Eng. C 2005, 25, 215-229.

332. Reis, R.L.; Cunha, A.M.; Allan, P.S.; Bevis, M.J. Structure development and control of injectionmolded hydroxylapatite-reinforced starch/EVOH composites. J. Polym. Adv. Tech. 1997, 16, 263-277.

333. Vaz, C.M.; Reis, R.L.; Cunha, A.M. Use of coupling agents to enhance the interfacial interactions in starch-EVOH/hydroxylapatite composites. Biomaterials 2002, 23, 629-635.

334. Leonor, I.B.; Ito, A.; Onuma, K.; Kanzaki, N.; Reis, R.L. In vitro bioactivity of starch thermoplastic/hydroxyapatite composite biomaterials: an in situ study using atomic force microscopy. Biomaterials 2003, 24, 579-585.

335. Vaz, C.M.; Reis, R.L.; Cunha, A.M. Degradation model of starch-EVOH+HA composites. Mater. Res. Innov. 2001, 4, 375-380.

336. Chen, L.J.; Wang, M. Production and evaluation of biodegradable composites based on PHB-PHV copolymer. Biomaterials 2002, 23, 2631-2639.

337. Ni, J.; Wang, M. In vitro evaluation of hydroxyapatite reinforced polyhydroxybutyrate composite. Mater. Sci. Eng. C 2002, 20, 101-109.

338. Carlo, E.C.; Borges, A.P.B.; Del Carlo, R.J.; Martinez, M.M.M.; Oliveira, P.M.; Morato, G.O.; Eleotério, R.B.; Reis, M.S. Comparison of in vivo properties of hydroxyapatite-polyhydroxybutyrate composites assessed for bone substitution. J. Craniofac. Surg. 2009, 20, 853-859.

339. Reis, E.C.C.; Borges, A.P.B.; Fonseca, C.C.; Martinez, M.M.M.; Eleotério, R.B.; Morato, G.O.; Oliveira, P.M. Biocompatibility, osteointegration, osteoconduction, and biodegradation of a hydroxyapatite-polyhydroxybutyrate composite. Braz. Arch. Biol. Technol. 2010, 53, 817-826. 
340. Sadat-Shojai, M.; Khorasani, M.T.; Jamshidi, A.; Irani, S. Nano-hydroxyapatite reinforced polyhydroxybutyrate composites: a comprehensive study on the structural and in vitro biological properties. Mater. Sci. Eng. C 2013, 33, 2776-2787.

341. Chen, D.Z.; Tang, C.Y.; Chan, K.C.; Tsui, C.P.; Yu, P.H.F.; Leung, M.C.P.; Uskokovic, P.S. Dynamic mechanical properties and in vitro bioactivity of PHBHV/HA nanocomposite. Compos. Sci. Technol. 2007, 67, 1617-1626.

342. Rai, B.; Noohom, W.; Kithva, P.H.; Grøndahl, L.; Trau, M. Bionanohydroxyapatite/poly(3hydroxybutyrate-co-3-hydroxyvalerate) composites with improved particle dispersion and superior mechanical properties. Chem. Mater. 2008, 20, 2802-2808.

343. Wang, Y.W.; Wu, Q.; Chen, J.; Chen, G.Q. Evaluation of three-dimensional scaffolds made of blends of hydroxyapatite and poly(3-hydroxybutyrate-co-3-hydroxyhexanoate) for bone reconstruction. Biomaterials 2005, 26, 899-904.

344. Linhart, W.; Lehmann, W.; Siedler, M.; Peters, F.; Schilling, A.F.; Schwarz, K.; Amling, M.; Rueger, J.M.; Epple, M. Composites of amorphous calcium phosphate and poly(hydroxybutyrate) and poly(hydroxybutyrate-co-hydroxyvalerate) for bone substitution: Assessment of the biocompatibility. J. Mater. Sci. 2006, 41, 4806-4813.

345. Azevedo, M.; Reis, R.L.; Claase, M.; Grijpma, D.; Feijen, J. Development and properties of polycaprolactone/hydroxyapatite composite biomaterials. J. Mater. Sci. Mater. Med. 2003, 14, 103-107.

346. Walsh, D.; Furuzono, T.; Tanaka, J. Preparation of porous composite implant materials by in situ polymerization of porous apatite containing $\varepsilon$-caprolactone or methyl methacrylate. Biomaterials 2001, 22, 1205-1212.

347. Kim, H.W. Biomedical nanocomposites of hydroxyapatite/polycaprolactone obtained by surfactant mediation. J. Biomed. Mater. Res. A 2007, 83, 169-177.

348. Heo, S.J.; Kim, S.E.; Wei, J.; Hyun, Y.T.; Yun, H.S.; Kim, D.H.; Shin, J.W.; Shin, J.W. Fabrication and characterization of novel nano- and micro-HA/PCL composite scaffolds using a modified rapid prototyping process. J. Biomed. Mater. Res. A 2009, 89A, 108-116.

349. Chuenjitkuntaworn, B.; Inrung, W.; Damrongsri, D.; Mekaapiruk, K.; Supaphol, P.; Pavasant, P. Polycaprolactone/hydroxyapatite composite scaffolds: preparation, characterization, and in vitro and in vivo biological responses of human primary bone cells. J. Biomed. Mater. Res. A 2010, 94, 241-251.

350. Bang, L.T.; Kawachi, G.; Nakagawa, M.; Munar, M.; Ishikawa, K.; Othman, R. The use of poly ( $\varepsilon$-caprolactone) to enhance the mechanical strength of porous Si-substituted carbonate apatite. J. Appl. Polym. Sci. 2013, 130, 426-433.

351. Mohd Yusoff, M.F.; Abdul Kadir, M.R.; Iqbal, N.; Hassan, M.A.; Hussain, R. Dipcoating of poly ( $\varepsilon$-caprolactone)/hydroxyapatite composite coating on Ti6Al4V for enhanced corrosion protection. Surf. Coat. Technol. 2014, 245, 102-107.

352. Kim, B.S.; Yang, S.S.; Lee, J. A polycaprolactone/cuttlefish bone-derived hydroxyapatite composite porous scaffold for bone tissue engineering. J. Biomed. Mater. Res. B Appl. Biomater. 2014, 102, 943-951. 
353. Causa, F.; Netti, P.A.; Ambrosio, L.; Ciapetti, G.; Baldini, N.; Pagani, S.; Martini, D.; Giunti, A. Poly-e-caprolactone/hydroxyapatite composites for bone regeneration: in vitro characterization and human osteoblast response. J. Biomed. Mater. Res. A 2006, 76, 151-162.

354. Thomas, V.; Jagani, S.; Johnson, K.; Jose, M.V.; Dean, D.R.; Vohra, Y.K.; Nyairo, E. Electrospun bioactive nanocomposite scaffolds of polycaprolactone and nanohydroxyapatite for bone tissue engineering. J. Nanosci. Nanotechol. 2006, 6, 487-493.

355. Marra, K.G.; Szem, J.W.; Kumta, P.N.; DiMilla, P.A.; Weiss, L.E. In vitro analysis of biodegradable polymer blend/hydroxyapatite composites for bone tissue engineering. J. Biomed. Mater. Res. 1999, 47, 324-335.

356. Dunn, A.; Campbell, P.; Marra, K.G. The influence of polymer blend composition on the degradation of polymer/hydroxyapatite biomaterials. J. Mater. Sci. Mater. Med. 2001, 12, 673-677.

357. Calandrelli, L.; Immirzi, B.; Malinconico, M.; Volpe, M.; Oliva, A.; Ragione, F. Preparation and characterization of composites based on biodegradable polymers for in vivo application. Polymer 2000, 41, 8027-8033.

358. Chen, B.; Sun, K. Poly( $\varepsilon$-caprolactone)/hydroxyapatite composites: effects of particle size, molecular weight distribution and irradiation on interfacial interaction and properties. Polym. Test. 2005, 24, 64-70.

359. Kim, H.W.; Knowles, J.C.; Kim, H.E. Hydroxyapatite/poly(e-caprolactone) composite coatings on hydroxyapatite porous bone scaffold for drug delivery. Biomaterials 2004, 25, 1279-1287.

360. Ural, E.; Kesenci, K.; Fambri, L.; Migliaresi, C.; Piskin, E. Poly(D,L-lactide/ع-caprolactone)/ hydroxyapatite composites. Biomaterials 2000, 21, 2147-2154.

361. Takayama, T.; Todo, M. Improvement of fracture properties of hydroxyapatite particle filled poly(L-lactide)/poly(e-caprolactone) biocomposites using lysine tri-isocyanate. J. Mater. Sci. 2010, 45, 6266-6270.

362. Kim, H.W.; Lee, E.J.; Kim, H.E.; Salih, V.; Knowles, J.C. Effect of fluoridation of hydroxyapatite in hydroxyapatite/polycaprolactone composites on osteoblast activity. Biomaterials 2005, 26, 4395-4404.

363. Gloria, A.; Russo, T.; D’Amora, U.; Zeppetelli, S.; D’Alessandro, T.; Sandri, M.; BañobreLópez, M.; Piñeiro-Redondo, Y.; Uhlarz, M.; Tampieri, A.; et al. Magnetic poly( $\varepsilon$-caprolactone)/ iron-doped hydroxyapatite nanocomposite substrates for advanced bone tissue engineering. $J . R$. Soc. Interface 2013, 10, doi:10.1098/rsif.2012.0833.

364. Shokrollahi, P.; Mirzadeh, H.; Scherman, O.A.; Huck, W.T.S. Biological and mechanical properties of novel composites based on supramolecular polycaprolactone and functionalized hydroxyapatite. J. Biomed. Mater. Res. A 2010, 95, 209-221.

365. Mehmanchi, M.; Shokrollahi, P.; Atai, M.; Omidian, H.; Bagheri, R. Supramolecular polycaprolactone nanocomposite based on functionalized hydroxyapatite. J. Bioact. Compat. Polym. 2012, 27, 467-480.

366. Busch, S.; Dolhaine, H.; DuChesne, A.; Heinz, S.; Hochrein, O.; Laeri, F.; Podebrad, O.; Vietze, U.; Weiland, T.; Kniep, R. Biomimetic morphogenesis of fluorapatite-gelatin composites: Fractal growth, the question of intrinsic electric fields, core/shell assemblies, hollow spheres and reorganization of denatured collagen. Eur. J. Inorg. Chem. 1999, 1999, 1643-1653. 
367. Simon, P.; Schwarz, U.; Kniep, R. Hierarchical architecture and real structure in a biomimetic nano-composite of fluorapatite with gelatine: A model system for steps in dentino- and osteogenesis? J. Mater. Chem. 2005, 15, 4992-4996.

368. Tlatlik, H.; Simon, P.; Kawska, A.; Zahn, D.; Kniep, R. Biomimetic fluorapatite-gelatin nanocomposites: Pre-structuring of gelatin matrices by ion impregnation and its effect on form development. Angew. Chem. Int. Ed. Engl. 2006, 45, 1905-1910.

369. Simon, P.; Zahn, D.; Lichte, H.; Kniep, R. Intrinsic electric dipole fields and the induction of hierarchical form developments in fluorapatite-gelatin nanocomposites: A general principle for morphogenesis of biominerals? Angew. Chem. Int. Ed. Engl. 2006, 45, 1911-1915.

370. Kniep, R.; Simon, P. "Hidden" hierarchy of microfibrils within 3D-periodic fluorapatite-gelatin nanocomposites: Development of complexity and form in a biomimetic system. Angew. Chem. Int. Ed. Engl. 2008, 47, 1405-1409.

371. Brickmann, J.; Paparcone, R.; Kokolakis, S.; Zahn, D.; Duchstein, P.; Carrillocabrera, W.; Simon, P.; Kniep, R. Fluorapatite-gelatine nanocomposite superstructures: New insights into a biomimetic system of high complexity. ChemPhysChem 2010, 11, 1851-1853.

372. Vyalikh, A.; Simon, P.; Rosseeva, E.; Buder, J.; Kniep, R.; Scheler, U. Intergrowth and interfacial structure of biomimetic fluorapatite-gelatin nanocomposite: a solid-state NMR study. J. Phys. Chem. B 2014, 118, 724-730.

373. Gashti, M.P.; Stir, M.; Hulliger, J. Synthesis of bone-like micro-porous calcium phosphate/iotacarrageenan composites by gel diffusion. Colloid Surface B 2013, 110, 426-433.

374. Behl, M.; Razzaq, M.Y.; Lendlein, A. Multifunctional shape-memory polymers. Adv. Mater. 2010, 22, 3388-3410.

375. Deng, C.; Wang, B.; Dongqin, X.; Zhou, S.; Duan, K.; Weng, J. Preparation and shape memory property of hydroxyapatite/poly (vinyl alcohol) composite. Polym. Plast. Technol. Eng. 2012, 51, 1315-1318.

376. Kutikov, A.B.; Reyer, K.A.; Song, J. Shape-memory performance of thermoplastic amphiphilic triblock copolymer poly(D,L-lactic acid-co-ethylene glycol-co-D,L-lactic acid) (PELA)/ hydroxyapatite composites. Macromol. Chem. Phys. 2014, 215, 2482-2490.

377. Wong, T.W.; Wahit, M.U.; Abdul Kadir, M.R.; Soheilmoghaddam, M.; Balakrishnan, H. A novel poly(xylitol-co-dodecanedioate)/hydroxyapatite composite with shape-memory behaviour. Mater. Lett. 2014, 126, 105-108.

378. Handschel, J.; Wiesmann, H.P.; Stratmann, U.; Kleinheinz, J.; Meyer, U.; Joos, U. TCP is hardly resorbed and not osteoconductive in a non-loading calvarial model. Biomaterials 2002, 23, 1689-1695.

379. Kikuchi, M.; Tanaka, J. Chemical interaction in $\beta$-tricalcium phosphate/copolymerized poly-Llactide composites. J. Ceram. Soc. Jpn. 2000, 108, 642-645.

380. Aunoble, S.; Clement, D.; Frayssinet, P.; Harmand, MF.; le Huec, J.C. Biological performance of a new $\beta$-TCP/PLLA composite material for applications in spine surgery: in vitro and in vivo studies. J. Biomed. Mater. Res. A 2006, 78, 416-422.

381. Haaparanta, A.M.; Haimi, S.; Ellä, V.; Hopper, N.; Miettinen, S.; Suuronen, R.; Kellomäki, M. Porous polylactide/ $\beta$-tricalcium phosphate composite scaffolds for tissue engineering applications. J. Tissue Eng. Regen. Med. 2010, 4, 366-373. 
382. Kikuchi, M.; Koyama, Y.; Yamada, T.; Imamura, Y.; Okada, T.; Shirahama, N.; Akita, K.; Takakuda, K.; Tanaka, J. Development of guided bone regeneration membrane composed of $\beta$-tricalcium phosphate and poly(L-lactide-co-glycolide-co- $\varepsilon$-caprolactone) composites. Biomaterials 2004, 25, 5979-5986.

383. Chen, T.M.; Yao, C.H.; Wang, H.J.; Chou, G.H.; Lee, T.W.; Lin, F.H. Evaluation of a novel malleable, biodegradable osteoconductive composite in a rabbit cranial defect model. Mater. Chem. Phys. 1998, 55, 44-50.

384. Dong, G.C.; Chen, H.M.; Yao, C.H. A novel bone substitute composite composed of tricalcium phosphate, gelatin and drynaria fortunei herbal extract. J. Biomed. Mater. Res. A 2008, 84, 167-177.

385. Ji, J.; Yuan, X.; Xia, Z.; Liu, P.; Chen, J. Porous $\beta$-tricalcium phosphate composite scaffold reinforced by $\mathrm{K}_{2} \mathrm{HPO}_{4}$ and gelatin. Key Eng. Mater. 2010, 434-435, 620-623.

386. Yao, C.H.; Liu, B.S.; Hsu, S.H.; Chen, Y.S.; Tsai, C.C. Biocompatibility and biodegradation of a bone composite containing tricalcium phosphate and genipin crosslinked gelatin. J. Biomed. Mater. Res. A 2004, 69, 709-717.

387. Eslaminejad, M.B.; Mirzadeh, H.; Mohamadi, Y.; Nickmahzar, A. Bone differentiation of marrow-derived mesenchymal stem cells using $\beta$-tricalcium phosphate-alginate-gelatin hybrid scaffolds. J. Tissue Eng. Regen. Med. 2007, 1, 417-424.

388. Takahashi, Y.; Yamamoto, M.; Tabata, Y. Osteogenic differentiation of mesenchymal stem cells in biodegradable sponges composed of gelatin and $\beta$-tricalcium phosphate. Biomaterials 2005, 26, 3587-3596.

389. Bigi, A.; Cantelli, I.; Panzavolta, S.; Rubini, K. $\alpha$-tricalcium phosphate-gelatin composite cements. J. Appl. Biomater. Biomech. 2004, 2, 81-87.

390. Yang, S.H.; Hsu, C.K.; Wang, K.C.; Hou, S.M.; Lin, F.H. Tricalcium phosphate and glutaraldehyde crosslinked gelatin incorporating bone morphogenetic protein-A viable scaffold for bone tissue engineering. J. Biomed. Mater. Res. B Appl. Biomater. 2005, 74, 468-475.

391. Kato, M.; Namikawa, T.; Terai, H.; Hoshino, M.; Miyamoto, S.; Takaoka, K. Ectopic bone formation in mice associated with a lactic acid/dioxanone/ethylene glycol copolymer-tricalcium phosphate composite with added recombinant human bone morphogenetic protein-2. Biomaterials 2006, 27, 3927-3933.

392. Muramatsu, K.; Oba, K.; Mukai, D.; Hasegawa, K.; Masuda, S.; Yoshihara, Y. Subacute systemic toxicity assessment of $\beta$-tricalcium phosphate/carboxymethyl-chitin composite implanted in rat femur. J. Mater. Sci. Mater. Med. 2007, 18, 513-522.

393. Panzavolta, S.; Fini, M.; Nicoletti, A.; Bracci, B.; Rubini, K.; Giardino, R.; Bigi, A. Porous composite scaffolds based on gelatin and partially hydrolyzed $\alpha$-tricalcium phosphate. Acta Biomater. 2009, 5, 636-643.

394. Uchino, T.; Kamitakahara, M.; Otsuka, M.; Ohtsuki, C. Hydroxyapatite-forming capability and mechanical properties of organic-inorganic hybrids and $\alpha$-tricalcium phosphate porous bodies. $J$. Ceram. Soc. Jpn. 2010, 118, 57-61.

395. Boguń, M.; Rabiej, S. The influence of fiber formation conditions on the structure and properties of nanocomposite alginate fibers containing tricalcium phosphate or montmorillonite. Polym. Compos. 2010, 31, 1321-1331. 
396. Park, C.H.; Kim, E.K.; Tijing, L.D.; Amarjargal, A.; Pant, H.R.; Kim, C.S.; Shon, H.K. Preparation and characterization of LA/PCL composite fibers containing beta tricalcium phosphate ( $\beta$-TCP) particles. Ceram. Int. 2014, 40, 5049-5054.

397. Ngamviriyavong, P.; Patntirapong, S.; Janvikul, W.; Arphavasin, S.; Meesap, P.; Singhatanadgit, W. Development of poly(butylene succinate)/calcium phosphate composites for bone engineering. Compos. Interface 2014, 21, 431-441.

398. Flauder, S.; Sajzew, R.; Müller, F.A. Mechanical properties of porous $\beta$-tricalcium phosphate composites prepared by ice-templating and poly( $\sigma$-caprolactone) impregnation. ACS Appl. Mater. Interf. 2015, 7, 845-851.

399. Agyemang, F.O.; Sheikh, F.A.; Appiah-Ntiamoah, R.; Chandradass, J.; Kim, H. Synthesis and characterization of poly (vinylidene fluoride)-calcium phosphate composite for potential tissue engineering applications. Ceram. Int. 2015, 41, 7066-7072.

400. Cohen, B.; Panker, M.; Zuckerman, E.; Foox, M.; Zilberman, M. Effect of calcium phosphate-based fillers on the structure and bonding strength of novel gelatin-alginate bioadhesives. J. Biomater. Appl. 2014, 28, 1366-1375.

401. Bleach, N.C.; Tanner, K.E.; Kellomäki, M.; Törmälä, P. Effect of filler type on the mechanical properties of self-reinforced polylactide-calcium phosphate composites. J. Mater. Sci. Mater. Med. 2001, 12, 911-915.

402. Liu, L.; Xiong, Z.; Yan, Y.N.; Hu, Y.Y.; Zhang, R.J.; Wang, S.G. Porous morphology, porosity, mechanical properties of poly( $\alpha$-hydroxy acid)-tricalcium phosphate composite scaffolds fabricated by low-temperature deposition. J. Biomed. Mater. Res. A 2007, 82, 618-629.

403. Zhang, Y.; Zhang, M.Q. Synthesis and characterization of macroporous chitosan/calcium phosphate composite scaffolds for tissue engineering. J. Biomed. Mater. Res. 2001, 55, 304-312.

404. Rai, B.; Teoh, S.H.; Hutmacher, D.W.; Cao, T.; Ho, K.H. Novel PCL-based honeycomb scaffolds as drug delivery systems for rhBMP-2. Biomaterials 2005, 26, 3739-3748.

405. Rai, B.; Teoh, S.H.; Ho, K.H.; Hutmacher, D.W.; Cao, T.; Chen, F.; Yacob, K. The effect of rhBMP-2 on canine osteoblasts seeded onto 3D bioactive polycaprolactone scaffolds. Biomaterials 2004, 25, 5499-5506.

406. Lei, Y.; Rai, B.; Ho, K.H.; Teoh, S.H. In vitro degradation of novel bioactive polycaprolactone - $20 \%$ tricalcium phosphate composite scaffolds for bone engineering. Mater. Sci. Eng. C 2007, 27, 293-298.

407. Miyai, T.; Ito, A.; Tamazawa, G.; Matsuno, T.; Sogo, Y.; Nakamura, C.; Yamazaki, A.; Satoh, T. Antibiotic-loaded poly- $\varepsilon$-caprolactone and porous $\beta$-tricalcium phosphate composite for treating osteomyelitis. Biomaterials 2008, 29, 350-358.

408. Li, Y.; Wu, Z.G.; Li, X.K.; Guo, Z.; Wu, S.H.; Zhang, Y.Q.; Shi, L.; Teoh, S.H.; Liu, Y.C.; Zhang, Z.Y. A polycaprolactone-tricalcium phosphate composite scaffold as an autograft-free spinal fusion cage in a sheep model. Biomaterials 2014, 35, 5647-5659.

409. Takahashi, Y.; Yamamoto, M.; Tabata, Y. Enhanced osteoinduction by controlled release of bone morphogenetic protein-2 from biodegradable sponge composed of gelatin and $\beta$-tricalcium phosphate. Biomaterials 2005, 26, 4856-4865. 
410. Ignatius, A.A.; Betz, O.; Augat, P.; Claes, L.E. In vivo investigations on composites made of resorbable ceramics and poly(lactide) used as bone graft substitutes. J. Biomed. Mater. Res. Appl. Biomater. 2001, 58, 701-709.

411. Miao, X.; Lim, W.K.; Huang, X.; Chen, Y. Preparation and characterization of interpenetrating phased TCP/HA/PLGA composites. Mater. Lett. 2005, 59, 4000-4005.

412. Dorozhkin, S.V. Biphasic, triphasic and multiphasic calcium orthophosphates. Acta Biomater. 2012, 8, 963-977.

413. Brodie, J.C.; Goldie, E.; Connel, G.; Merry, J.; Grant, M.H. Osteoblast interactions with calcium phosphate ceramics modified by coating with type I collagen. J. Biomed. Mater. Res. A 2005, 73, 409-421.

414. Zhang, L.F.; Sun, R.; Xu, L.; Du, J.; Xiong, Z.C.; Chen, H.C.; Xiong, C.D. Hydrophilic poly (ethylene glycol) coating on PDLLA/BCP bone scaffold for drug delivery and cell culture. Mater. Sci. Eng. C 2008, 28, 141-149.

415. Ignjatovic, N.; Ninkov, P.; Ajdukovic, Z.; Konstantinovic, V.; Uskokovic, D. Biphasic calcium phosphate/poly-(D,L-lactide-co-glycolide) biocomposite as filler and blocks for reparation of bone tissue. Mater. Sci. Forum 2005, 494, 519-524.

416. Ignjatovic, N.; Ninkov, P.; Ajdukovic, Z.; Vasiljevic-Radovic, D.; Uskokovic, D. Biphasic calcium phosphate coated with poly-D,L-lactide-co-glycolide biomaterial as a bone substitute. $J$. Eur. Ceram. Soc. 2007, 27, 1589-1594.

417. Yang, W.; Yin, G.; Zhou, D.; Gu, J.; Li, Y. In vitro characteristics of surface-modified biphasic calcium phosphate/poly(L-Lactide) biocomposite. Adv. Eng. Mater. 2010, 12, B128-B132.

418. Ignjatovic, N.; Ninkov, P.; Kojic, V.; Bokurov, M.; Srdic, V.; Krnojelac, D.; Selakovic, S.; Uskokovic, D. Cytotoxicity and fibroblast properties during in vitro test of biphasic calcium phosphate/poly-D,L-lactide-co-glycolide biocomposites and different phosphate materials. Microsc. Res. Tech. 2006, 69, 976-982.

419. Ajdukovic, Z.; Ignjatovic, N.; Petrovic, D.; Uskokovic, D. Substitution of osteoporotic alveolar bone by biphasic calcium phosphate/poly-D,L-lactide-co-glycolide biomaterials. J. Biomater. Appl. 2007, 21, 317-328.

420. Kim, H.W.; Knowles, J.C.; Kim, H.E. Effect of biphasic calcium phosphates on drug release and biological and mechanical properties of poly( $\varepsilon$-caprolactone) composite membranes. J. Biomed. Mater. Res. A 2004, 70, 467-479.

421. Kwak, K.A.; Jyoti, M.A.; Song, H.Y. In vitro and in vivo studies of three dimensional porous composites of biphasic calcium phosphate/poly $\varepsilon$-caprolactone: effect of bio-functionalization for bone tissue engineering. Appl. Surf. Sci. 2014, 301, 307-314.

422. Van Leeuwen, A.C.; Yuan, H.; Passanisi, G.; van der Meer, J.W.; de Bruijn, J.D.; van Kooten, T.G.; Grijpma, D.W.; Bos, R.R. Poly(trimethylene carbonate) and biphasic calcium phosphate composites for orbital floor reconstruction: A feasibility study in sheep. Eur. Cell. Mater. 2014, 27, 81-97.

423. Bakhtiari, L.; Rezai, H.R.; Hosseinalipour, S.M.; Shokrgozar, M.A. Investigation of biphasic calcium phosphate/gelatin nanocomposite scaffolds as a bone tissue engineering. Ceram. Int. 2010, 36, 2421-2426. 
424. Bakhtiari, L.; Rezai, H.R.; Hosseinalipour, S.M.; Shokrgozar, M.A. Preparation of porous Biphasic calcium phosphate-gelatin nanocomposite for bone tissue engineering. J. Nano Res. 2010, 11, 67-72.

425. Matsuda, A.; Ikoma, T.; Kobayashi, H.; Tanaka, J. Preparation and mechanical property of coreshell type chitosan/calcium phosphate composite fiber. Mater. Sci. Eng. C 2004, 24, 723-728.

426. Rattanachan, S.; Lorprayoon, C.; Boonphayak, P. Synthesis of chitosan/brushite powders for bone cement composites. J. Ceram. Soc. Jpn. 2008, 116, 36-41.

427. Ohsawa, H.; Ito, A.; Sogo, Y.; Yamazaki, A.; Ohno, T. Synthesis of albumin/DCP nanocomposite particles. Key Eng. Mater. 2007, 330-332, 239-242.

428. Xu, H.H.K.; Sun, L.; Weir, M.D.; Antonucci, J.M.; Takagi, S.; Chow, L.C.; Peltz, M. Nano DCPA-whisker composites with high strength and $\mathrm{Ca}$ and $\mathrm{PO}_{4}$ release. J. Dent. Res. 2006, 85, $722-727$.

429. Xu, H.H.K.; Weir, M.D.; Sun, L.; Takagi, S.; Chow, L.C. Effects of calcium phosphate nanoparticles on Ca-PO4 composite. J. Dent. Res. 2007, 86, 378-383.

430. Xu, H.H.K.; Weir, M.D.; Sun, L. Nanocomposites with $\mathrm{Ca}$ and $\mathrm{PO}_{4}$ release: Effects of reinforcement, dicalcium phosphate particle size and silanization. Dent. Mater. 2007, 23, 1482-1491.

431. Chen, W.C.; Chang, K.C.; Wu, H.Y.; Ko, C.L.; Huang, C.L. Thermal cycling effect of dicalcium phosphate-reinforced composites on auto-mineralized dental resin. Mater. Sci. Eng. C 2014, 45, 359-368.

432. Tortet, L.; Gavarri, J.R.; Nihoul, G.; Dianoux, A.J. Proton mobilities in brushite and brushite/polymer composites. Solid State Ionics 1997, 97, 253-256.

433. Tortet, L.; Gavarri, J.R.; Musso, J.; Nihoul, G.; Sarychev, A.K. Percolation and modeling of proton conduction in polymer/brushite composites. J. Solid State Chem. 1998, 141, 392-403.

434. Dorozhkin, S.V. Amorphous calcium orthophosphates: nature, chemistry and biomedical applications. Int. J. Mater. Chem. 2012, 2, 19-46.

435. Dorozhkin, S.V. Calcium orthophosphates in dentistry. J. Mater. Sci. Mater. Med. 2013, 24, 1335-1363.

436. Gutierrez, M.C.; Jobbágy, M.; Ferrer, M.L.; del Monte, F. Enzymatic synthesis of amorphous calcium phosphate-chitosan nanocomposites and their processing into hierarchical structures. Chem. Mater. 2008, 20, 11-13.

437. Hakimimehr, D.; Liu, D.M.; Troczynski, T. In-situ preparation of poly(propylene fumarate)hydroxyapatite composite. Biomaterials 2005, 26, 7297-7303.

438. Antonucci, J.M.; Regnault, W.F.; Skrtic, D. Polymerization shrinkage and stress development in amorphous calcium phosphate/urethane dimethacrylate polymeric composites. J. Compos. Mater. 2010, 44, 355-367.

439. Wang, K.W.; Zhu, Y.J.; Chen, F.; Cao, S.W. Calcium phosphate/block copolymer hybrid porous nanospheres: preparation and application in drug delivery. Mater. Lett. 2010, 64, 2299-2301.

440. Cao, S.W.; Zhu, Y.J.; Wu, J.; Wang, K.W.; Tang, Q.L. Preparation and sustained-release property of triblock copolymer/calcium phosphate nanocomposite as nanocarrier for hydrophobic drug. Nanoscale Res. Lett. 2010, 5, 781-785.

441. Suzuki, O. Octacalcium phosphate (OCP)-based bone substitute materials. Jpn. Dent. Sci. Rev. 2013, 49, 58-71. 
442. Dorozhkin, S.V. Self-setting calcium orthophosphate formulations. J. Funct. Biomater. 2013, 4, 209-311.

443. Barralet, J.A.; Hofmann, M.; Grover, L.M.; Gbureck, U. High-strength apatitic cement by modification with $\alpha$-hydroxyacid salts. Adv. Mater. 2003, 15, 2091-2094.

444. Lou, C.W.; Huang, C.C.; Chen, W.C.; Hu, J.J.; Lu, C.T.; Lin, J.H. Preliminary studies in composite scaffolds of calcium phosphate bone cement with polylactide. Appl. Mech. Mater. 2012, 184-185, 1098-1101.

445. Zhong, M.L.; Chen, X.Q.; Fan, H.S.; Zhang, X.D. Incorporation of salmon calcitonin-loaded poly(lactide-co-glycolide) (PLGA) microspheres into calcium phosphate bone cement and the biocompatibility evaluation in vitro. J. Bioact. Compat. Polym. 2012, 27, 133-147.

446. Bigi, A.; Bracci, B.; Panzavolta, S. Effect of added gelatin on the properties of calcium phosphate cement. Biomaterials 2004, 25, 2893-2899.

447. Bigi, A.; Panzavolta, S.; Sturba, L.; Torricelli, P.; Fini, M.; Giardino, R. Normal and osteopenic bone-derived osteoblast response to a biomimetic gelatin-calcium phosphate bone cement. $J$. Biomed. Mater. Res. A 2006, 78, 739-745.

448. Panzavolta, S.; Torricelli, P.; Sturba, L.; Bracci, B.; Giardino, R.; Bigi, A. Setting properties and in vitro bioactivity of strontium-enriched gelatin-calcium phosphate bone cements. J. Biomed. Mater. Res. A 2008, 84, 965-972.

449. Maazouz, Y.; Montufar, E.B.; Guillem-Marti, J.; Fleps, I.; Öhman, C.; Persson, C.; Ginebra, M.P. Robocasting of biomimetic hydroxyapatite scaffolds using self-setting inks. J. Mater. Chem. B 2014, 2, 5378-5386.

450. Rammelt, S.; Neumann, M.; Hanisch, U.; Reinstorf, A.; Pompe, W.; Zwipp, H.; Biewener, A. Osteocalcin enhances bone remodeling around hydroxyapatite/collagen composites. J. Biomed. Mater. Res. A 2005, 73A, 284-294.

451. Park, J.H.; Lee, E.J.; Knowles, J.C.; Kim, H.W. Preparation of in situ hardening composite microcarriers: Calcium phosphate cement combined with alginate for bone regeneration. $J$. Biomater. Appl. 2014, 28, 1079-1084.

452. Wang, X.; Ma, J.; Feng, Q.; Cui, F.Z. Skeletal repair in rabbits with calcium phosphate cements incorporated phosphorylated chitin. Biomaterials 2002, 23, 4591-4600.

453. Xue, B.; Zhang, C.; Wang, Y.; Wang, J.; Zhang, J.; Lu, M.; Li, G.; Cao, Z.; Huang, Q. A novel controlled-release system for antibacterial enzyme lysostaphin delivery using hydroxyapatite/ chitosan composite bone cement. PLoS ONE 2014, 9, e113797.

454. Cao, C.; Li, H.; Li, J.; Liu, C.; Yang, H.; Li, B. Mechanical reinforcement of injectable calcium phosphate cement/silk fibroin (SF) composite by mineralized SF. Ceram. Int. 2014, 40, 13987-13993.

455. Liu, W.; Zhang, J.; Rethore, G.; Khairoun, K.; Pilet, P.; Tancret, F.; Bouler, J.M.; Weiss, P. A novel injectable, cohesive and toughened Si-HPMC (silanized-hydroxypropyl methylcellulose) composite calcium phosphate cement for bone substitution. Acta Biomater. 2014, 10, 3335-3345.

456. Sadiasa, A.; Sarkar, S.K.; Franco, R.A.; Min, Y.K.; Lee, B.T. Bioactive glass incorporation in calcium phosphate cement-based injectable bone substitute for improved in vitro biocompatibility and in vivo bone regeneration. J. Biomater. Appl. 2014, 28, 739-756. 
457. Perez, R.A.; Patel, K.D.; Kim, H.W. Novel magnetic nanocomposite injectables: Calcium phosphate cements impregnated with ultrafine magnetic nanoparticles for bone regeneration. $R S C$ Adv. 2015, 5, 13411-13419.

458. Canal, C.; Ginebra, M.P. Fibre-reinforced calcium phosphate cements: A review. J. Mech. Behav. Biomed. Mater. 2011, 4, 1658-1671.

459. Hasan, M.S.; Carpenter, N.; Wei, T.L.; McNally, D.; Ahmed, I.; Boszczyk, B.M. Effects of adding resorbable phosphate glass fibres and PLA to calcium phosphate bone cements. J. Appl. Biomater. Funct. Mater. 2014, 12, 203-209.

460. Low, K.L.; Tan, S.H.; Zein, S.H.S.; McPhail, D.S.; Boccaccini, A.R. Optimization of the mechanical properties of calcium phosphate/multi-walled carbon nanotubes/bovine serum albumin composites using response surface methodology. Mater. Des. 2011, 32, 3312-3319.

461. Chew, K.K.; Low, K.L.; Zein, S.H.S.; McPhail, D.S.; Gerhardt, L.C.; Roether, J.A.; Boccaccini, A.R. Reinforcement of calcium phosphate cement with multiwalled carbon nanotubes and bovine serum albumin for injectable bone substitute applications. J. Mech. Behav. Biomed. Mater. 2011, 4, 331-339.

462. Kim, S.B.; Kim, Y.J.; Yoon, T.L.; Park, S.A.; Cho, I.H.; Kim, E.J.; Kim, I.A.; Shin, J.W. The characteristics of a hydroxyapatite-chitosan-PMMA bone cement. Biomaterials 2004, 25, 5715-5723.

463. Vallo, C.I.; Montemartini, P.E.; Fanovich, M.A.; Lópes, J.M.P.; Cuadrado, T.R. Polymethylmethacrylate-based bone cement modified with hydroxyapatite. J. Biomed. Mater. Res. B Appl. Biomater. 1999, 48, 150-158.

464. Moursi, A.M.; Winnard, A.V.; Winnard, P.L.; Lannutti, J.J.; Seghi, R.R. Enhanced osteoblast response to a PMMA-HA composite. Biomaterials 2002, 23, 133-144.

465. Dalby, MJ.; di Silvio, L.; Harper, E.J.; Bonfield, W. Increasing hydroxyapatite incorporation into poly(methylmethacrylate) cement increases osteoblast adhesion and response. Biomaterials 2002, 23, 569-576.

466. Itokawa, H.; Hiraide, T.; Moriya, M.; Fujimoto, M.; Nagashima, G.; Suzuki, R.; Fujimoto, T. A 12 month in vivo study on the response of bone to a hydroxyapatite-polymethylmethacrylate cranioplasty composite. Biomaterials 2007, 28, 4922-4927.

467. Tham, W.L.; Chow, W.S.; Ishak, Z.A.M. Flexural and morphological properties of poly(methyl methacrylate)/hydroxyapatite composites: effects of planetary ball mill grinding time. J. Reinf. Plastics Compos. 2010, 29, 2065-2075.

468. Pattanayak, D.K.; Rao, B.T.; Mohan, T.R.R. Calcium phosphate bioceramics and bioceramic composites. J. Sol Gel Sci. Technol. 2011, 59, 432-447.

469. Arabmotlagh, M.; Bachmaier, S.; Geiger, F.; Rauschmann, M. PMMA-hydroxyapatite composite material retards fatigue failure of augmented bone compared to augmentation with plain PMMA: In vivo study using a sheep model. J. Biomed. Mater. Res. B Appl. Biomater. 2014, 102, 1613-1619.

470. Rao, M.; Su, Q.; Liu, Z.; Liang, P.; Wu, N.; Quan, C.; Jiang, Q. Preparation and characterization of a poly(methyl methacrylate) based composite bone cement containing poly(acrylate-co-silane) modified hydroxyapatite nanoparticles. J. Appl. Polym. Sci. 2014, 131, doi:10.1002/app.40587. 
471. Harper, E.J.; Behiri, J.C.; Bonfield, W. Flexural and fatigue properties of a bone cement based upon polyethylmethacrylate and hydroxyapatite. J. Mater. Sci. Mater. Med. 1995, 6, 799-803.

472. Arnold, J.C.; Venditti, N.P. Prediction of the long-term creep behaviour of hydroxyapatite-filled polyethylmethacrylate bone cements. J. Mater. Sci. Mater. Med. 2007, 18, 1849-1858.

473. Ohgaki, M.; Yamashita, K. Preparation of polymethylmethacrylate-reinforced functionally graded hydroxyapatite composites. J. Am. Ceram. Soc. 2003, 86, 1440-1442.

474. Del Real, RP.; Padilla, S.; Vallet-Regi, M. Gentamicin release from hydroxyapatite/poly(ethyl methacrylate)/poly(methyl methacrylate)composites. J. Biomed. Mater. Res. 2000, 52, 1-7.

475. Saito, M.; Maruoka, A.; Mori, T.; Sugano, N.; Hino, K. Experimental studies on a new bioactive bone cement: hydroxyapatite composite resin. Biomaterials 1994, 15, 156-160.

476. Kawagoe, K.; Saito, M.; Shibuya, T.; Nakashima, T.; Hino, K.; Yoshikawa, H. Augmentation of cancellous screw fixation with hydroxyapatite composite resin (CAP) in vivo. J. Biomed. Mater. Res. 2000, 53, 678-684.

477. Turner, A.W.L.; Gillies, R.M.; Svehla, M.J.; Saito, M.; Walsh, W.R. Hydroxyapatite composite resin cement augmentation of pedicle screw fixation. Clin. Orthop. Rel. Res. 2003, 406, 253-261.

478. Watson, K.E.; Ten Huisen, K.S.; Brown, P.W. The formation of hydroxyapatite-calcium polyacrylate composites. J. Mater. Sci. Mater. Med. 1999, 10, 205-213.

479. Reed, C.S.; Ten Huisen, K.S.; Brown, P.W.; Allcock, H.R. Thermal stability and compressive strength of calcium-deficient hydroxyapatite-poly[bis(carboxylatophenoxy)phosphazene] composites. Chem. Mater. 1996, 8, 440-447.

480. Peter, S.J.; Kim, P.; Yasko, A.W.; Yaszemski, M.J.; Mikos, A.G. Crosslinking characteristics of an injectable poly(propylene fumarate)/ $\beta$-tricalcium phosphate paste and mechanical properties of the crosslinked composite for use as a biodegradable bone cement. J. Biomed. Mater. Res. 1999, 44, 314-321.

481. He, S.; Yaszemski, M.J.; Yasko, A.W.; Engel, P.S.; Mikos, A.G. Injectable biodegradable polymer composites based on poly (propylene fumarate) crosslinked with poly(ethylene glycol)-dimethacrylate. Biomaterials 2000, 21, 2389-2394.

482. Ignjatovic, N.; Jovanovic, J.; Suljovrujic, E.; Uskokovic, D. Injectable polydimethylsiloxane/ hydroxyapatite composite cement. Biomed. Mater. Eng. 2003, 13, 401-410.

483. Fei, Z.; Hu, Y.; Wu, D.; Wu, H.; Lu, R.; Bai, J.; Song, H. Preparation and property of a novel bone graft composite consisting of rhBMP-2 loaded PLGA microspheres and calcium phosphate cement. J. Mater. Sci. Mater. Med. 2008, 19, 1109-1116.

484. Link, D.P.; van den Dolder, J.; van den Beucken, J.J.J.P.; Cuijpers, V.M.; Wolke, J.G.C.; Mikos, A.G.; Jansen, J.A. Evaluation of the biocompatibility of calcium phosphate cement/PLGA microparticle composites. J. Biomed. Mater. Res. A 2008, 87, 760-769.

485. Chiang, T.Y.; Ho, C.C.; Chen, D.C.H.; Lai, M.H.; Ding, S.J. Physicochemical properties and biocompatibility of chitosan oligosaccharide/gelatin/calcium phosphate hybrid cements. Mater. Chem. Phys. 2010, 120, 282-288.

486. Fujishiro, Y.; Takahashi, K.; Sato, T. Preparation and compressive strength of $\alpha$-tricalcium phosphate/gelatin gel composite cement. J. Biomed. Mater. Res. 2001, 54, 525-230.

487. Miyazaki, K.; Horibe, T.; Antonucci, J.M.; Takagi, S.; Chow, L.C. Polymeric calcium phosphate cements: Analysis of reaction products and properties. Dental Mater. 1993, 9, 41-45. 
488. Miyazaki, K.; Horibe, T.; Antonucci, J.M.; Takagi, S.; Chow, L.C. Polymeric calcium phosphate cements: Setting reaction modifiers. Dental Mater. 1993, 9, 46-50.

489. dos Santos, LA.; de Oliveira, L.C.; Rigo, E.C.S.; Carrodeguas, R.G.; Boschi, A.O.; de Arruda, A.C.F. Influence of polymeric additives on the mechanical properties of $\alpha$-tricalcium phosphate cement. Bone 1999, 25, 99S-102S.

490. Greish, Y.E.; Brown, P.W.; Bender, J.D.; Allcockm H.R.; Lakshmim S.; Laurencin, C.T. Hydroxyapatite-polyphosphazane composites prepared at low temperatures. J. Am. Ceram. Soc. 2007, 90, 2728-2734.

491. Greish, Y.E.; Bender, J.D.; Lakshmi, S.; Brown, P.W.; Allcock, H.R.; Laurencin, C.T. Formation of hydroxyapatite-polyphosphazene polymer composites at physiologic temperature. J. Biomed. Mater. Res. A 2006, 77, 416-425.

492. Greish, Y.E.; Bender, J.D.; Lakshmi, S.; Brown, P.W.; Allcock, H.R.; Laurencin, C.T. Low temperature formation of hydroxyapatite-poly(alkyl oxybenzoate) phosphazene composites for biomedical applications. Biomaterials 2005, 26, 1-9.

493. Mickiewicz, R.A.; Mayes, A.M.; Knaack, D. Polymer-calcium phosphate cement composites for bone substitutes. J. Biomed. Mater. Res. 2002, 61, 581-592.

494. Carey, L.E.; Xu, H.H.K.; Simon, C.G.; Takagi, S.; Chow, L.C. Premixed rapid-setting calcium phosphate composites for bone repair. Biomaterials 2005, 26, 5002-5014.

495. Krüger, R.; Seitz, J.M.; Ewald, A.; Bach, F.W.; Groll, J. Strong and tough magnesium wire reinforced phosphate cement composites for load-bearing bone replacement. J. Mech. Behav. Biomed. Mater. 2013, 20, 36-44.

496. Miao, X.; Tan, L.P.; Tan, L.S.; Huang, X. Porous calcium phosphate ceramics modified with PLGA-bioactive glass. Mater. Sci. Eng. C 2007, 27, 274-279.

497. Lickorish, D.; Guan, L.; Davies, J.E. A three-phase, fully resorbable, polyester/calcium phosphate scaffold for bone tissue engineering: Evolution of scaffold design. Biomaterials 2007, 28, $1495-1502$.

498. Iwasakia, Y.; Takahataa, Y.; Fujii, S. Self-setting particle-stabilized emulsion for hard-tissue engineering. Colloids Surf. B 2015, 126, 394-400.

499. Xu, H.H.K.; Simon, C.G. Fast setting calcium phosphate-chitosan scaffold: Mechanical properties and biocompatibility. Biomaterials 2005, 26, 1337-1348.

500. Zhang, L.; Li, Y.; Zhou, G.; Lu, GY.; Zuo, Y. Setting mechanism of nano-hydroxyapatite/ chitosan bone cement. J. Inorg. Mater. 2006, 21, 1197-1202.

501. Ruhe, P.Q.; Hedberg, E.L.; Padron, N.T.; Spauwen, P.H.M.; Jansen, J.A.; Mikos, A.G. Biocompatibility and degradation of poly(D,L-lactic-co-glycolic acid)/calcium phosphate cement composites. J. Biomed. Mater. Res. A 2005, 74, 533-544.

502. Guo, D.G.; Sun, H.L.; Xu, K.W.; Han, Y. Long-term variations in mechanical properties and in vivo degradability of CPC/PLGA composite. J. Biomed. Mater. Res. B Appl. Biomater. 2007, $82,533-544$.

503. Habraken, W.J.E.M.; Wolke, J.G.C.; Mikos, A.G.; Jansen, J.A. Injectable PLGA microsphere/calcium phosphate cements: physical properties and degradation characteristics. J. Biomater. Sci. Polym. Edn. 2006, 17, 1057-1074. 
504. Ruhe, P.Q.; Hedberg-Dirk, E.L.; Padron, N.T.; Spauwen, P.H.M.; Jansen, J.A.; Mikos, A.G. Porous poly (D,L-lactic-co-glycolic acid)/calcium phosphate cement composite for reconstruction of bone defects. Tissue Eng. 2006, 12, 789-800.

505. Ruhe, P.Q.; Hedberg, E.L.; Padron, N.T.; Spauwen, P.H.M.; Jansen, J.A.; Mikos, A.G. rhBMP2 release from injectable poly (D,L-lactic-co-glycolic acid)/calcium phosphate composites. $J$. Bone Joint Surg. (Am.) 2003, 85A, 75-81.

506. Ruhe, P.Q.; Boerman, O.C.; Russel, F.G.M.; Spauwen, P.H.M.; Mikos, A.G.; Jansen, J.A. Controlled release of rhBMP-2 loaded poly (D,L-lactic-co-glycolic acid)/calcium phosphate cement composites in vivo. J. Control. Release 2005, 106, 162-171.

507. Plachokova, A.; Link. D.; van den Dolder. J.; van den Beucken. J.; Jansen, J.A. Bone regenerative properties of injectable PGLA-CaP composite with TGF- $\beta 1$ in a rat augmentation model. J. Tissue Eng. Regen. Med. 2007, 1, 457-464.

508. Sato, S.; Koshino, T.; Saito, T. Osteogenic response of rabbit tibia to hydroxyapatite particle_-Plaster of Paris mixture. Biomaterials 1998, 19, 1895-1900.

509. Chen, W.L.; Chen, C.K.; Lee, J.W.; Lee, Y.L.; Ju, C.P.; Ju, C.P.; Lin, J.H.C. Structure, properties and animal study of a calcium phosphate/calcium sulfate composite cement. Mater. Sci. Eng. C 2014, 37, 60-67.

510. Zhou, S.; Ma, J.; Shen, Y.; Haapasalo, M.; Ruse, N.D.; Yang, Q.; Troczynski, T. In vitro studies of calcium phosphate silicate bone cements. J. Mater. Sci. Mater. Med. 2013, 24, 355-364.

511. Motisuke, M.; Santos, V.R.; Bazanini, N.C.; Bertran, C.A. Apatite bone cement reinforced with calcium silicate fibers. J. Mater. Sci. Mater. Med. 2014, 25, 2357-2363.

512. Correa, D.; Almirall, A.; Carrodeguas, R.G.; dos Santos, L.A.; de Aza, A.H.; Parra, J.; Morejon, L.; Delgado, J.A. $\alpha$-Tricalcium phosphate cements modified with $\beta$-dicalcium silicate and tricalcium aluminate: physicochemical characterization, in vitro bioactivity and cytotoxicity. J. Biomed. Mater. Res. B Appl. Biomater. 2015, 103, 72-83.

513. Morejón-Alonso, L.; Motisuke, M.; Correa, J.R.; Carrodeguas, R.G.; dos Santos, L.A. In situ synchrotron X-ray powder diffraction study of the early hydration of $\alpha$-tricalcium phosphate/tricalcium silicate composite bone cement. Mater. Res. 2015, 18, 164-169.

514. Webster, T.J. Nanophase ceramics: the future orthopedic and dental implant material. Adv. Chem. Eng. 2001, 27, 125-166.

515. Webster, T.J.; Ergun, C.; Doremus, R.H.; Siegel, R.W.; Bizios, R. Enhanced functions of osteoblasts on nanophase ceramics. Biomaterials 2000, 21, 1803-1810.

516. Zakaria, S.M.; Zein, S.H.S.; Othman, M.R.; Yang, F.; Jansen, J.A. Nanophase hydroxyapatite as a biomaterial in advanced hard tissue engineering: A review. Tissue Eng. B 2013, 19, 431-441.

517. Dorozhkin, S.V. Nanodimensional and nanocrystalline calcium orthophosphates. Int. J. Chem. Mater. Sci. 2013, 1, 105-174.

518. Li, G.; Huang, J.; Li, Y.; Zhang, R.; Deng, B.; Zhang, J.; Aoki, H. In vitro study on influence of a discrete nano-hydroxyapatite on leukemia P388 cell behavior. Biomed. Mater. Eng. 2007, 17, 321-327.

519. Tadic, D.; Peters, F.; Epple, M. Continuous synthesis of amorphous carbonated apatites. Biomaterials 2002, 23, 2553-2559. 
520. Xu, H.H.K.; Sun, L.; Weir, M.D.; Takagi, S.; Chow, L.C.; Hockey, B. Effects of incorporating nanosized calcium phosphate particles on properties of whisker-reinforced dental composites. J. Biomed. Mater. Res. B Appl. Biomater. 2007, 81, 116-125.

521. Shu, X.; Shi, Q.; Feng, J.; Xie, X.; Chen, Y. Design and in vitro evaluation of novel $\gamma$-PGA/hydroxyapatite nanocomposites for bone tissue engineering. J. Mater. Sci. 2014, 49, 7742-7749.

522. Hong, Z.K.; Zhang, P.B.; He, C.L.; Qiu, X.Y.; Liu, A.X.; Chen, L.; Chen. X.; Jing, X. Nanocomposite of poly(L-lactide) and surface grafted hydroxyapatite: mechanical properties and biocompatibility. Biomaterials 2005, 26, 6296-6304.

523. Deng, C.; Weng, J.; Cheng, QY.; Zhou, S.B.; Lu, X.; Wan, J.X.; Qu, S.X.; Feng, B.; Li, X.H. Choice of dispersants for the nano-apatite filler of polylactide-matrix composite biomaterial. Curr. Appl. Phys. 2007, 7, 679-682.

524. Deng, C.; Weng, J.; Lu, X.; Zhou, S.B.; Wan, J.X.; Qu, S.X.; Feng, B.; Li. X.H.; Cheng, Q.Y. Mechanism of ultrahigh elongation rate of poly(D,L-lactide)-matrix composite biomaterial containing nano-apatite fillers. Mater. Lett. 2008, 62, 607-610.

525. Kothapalli, C.R.; Shaw, M.T.; Wei, M. Biodegradable HA-PLA 3-D porous scaffolds: Effect of nano-sized filler content on scaffold properties. Acta Biomater. 2005, 1, 653-662.

526. Hong, Z.; Qiu, X.; Sun, J.; Deng, M.; Chen, X.; Jing, X. Grafting polymerization of L-lactide on the surface of hydroxyapatite nano-crystals. Polymer 2004, 45, 6699-6706.

527. Xiao, Y.; Li, D.; Fan, H.; Li, X.; Gu, Z.; Zhang, X. Preparation of nano-HA/PLA composite by modified-PLA for controlling the growth of HA crystals. Mater. Lett. 2007, 61, 59-62.

528. Qiu, X.; Han, Y.; Zhuang, X.; Chen, X.; Li, Y.; Jing, X. Preparation of nano-hydroxyapatite/ poly(L-lactide) biocomposite microspheres. J. Nanopart. Res. 2007, 9, 901-908.

529. Deplaine, H.; Ribelles, J.L.G.; Ferrer, G.G. Effect of the content of hydroxyapatite nanoparticles on the properties and bioactivity of poly(L-lactide)-hybrid membranes. Compos. Sci. Technol. 2010, 70, 1805-1812.

530. Aydin, E.; Planell, J.A.; Hasirci, V. Hydroxyapatite nanorod-reinforced biodegradable poly(L-lactic acid) composites for bone plate applications. J. Mater. Sci. Mater. Med. 2011, 22, 2413-2427.

531. Bianco, A.; Bozzo, B.M.; Del Gaudio, C.; Cacciotti, I.; Armentano, I.; Dottori, M.; D’Angelo, F.; Martino, S.; Orlacchio, A.; Kenny, J.M. Poly (L-lactic acid)/calcium-deficient nanohydroxyapatite electrospun mats for bone marrow stem cell cultures. J. Bioact. Compat. Polym. 2011, 26, 225-241.

532. Chae, T.; Yang, H.; Ko, F.; Troczynski, T. Bio-inspired dicalcium phosphate anhydrate/poly(lactic acid) nanocomposite fibrous scaffolds for hard tissue regeneration: In situ synthesis and electrospinning. J. Biomed. Mater. Res. A 2014, 10, 514-522.

533. Liu, Z.; Chen, Y.; Ding, W.; Zhang, C. Filling behavior, morphology evolution and crystallization behavior of microinjection molded poly(lactic acid)/hydroxyapatite nanocomposites. Composites $A$ 2015, 72, 85-95.

534. Hong, Z.; Zhang, P.; Liu, A.; Chen, L.; Chen, X.; Jing, X. Composites of poly(lactide-coglycolide) and the surface modified carbonated hydroxyapatite nanoparticles. J. Biomed. Mater. Res. A 2007, 81, 515-522. 
535. Huang, Y.X.; Ren, J.; Chen, C.; Ren, T.B.; Zhou, X.Y. Preparation and properties of poly(lactideco-glycolide) (PLGA)/nano-hydroxyapatite (NHA) scaffolds by thermally induced phase separation and rabbit MSCs culture on scaffolds. J. Biomater. Appl. 2008, 22, 409-432.

536. Xue, D.; Zheng, Q.; Zong, C.; Li, Q.; Li, H.; Qian, S.; Zhang, B.; Yu, L.; Pan, Z. Osteochondral repair using porous poly(lactide-co-glycolide)/nano-hydroxyapatite hybrid scaffolds with undifferentiated mesenchymal stem cells in a rat model. J. Biomed. Mater. Res. A 2010, 94, 259-270.

537. Torabinejad, B.; Mohammadi-Rovshandeh, J.; Davachi, S.M.; Zamanian, A. Synthesis and characterization of nanocomposite scaffolds based on triblock copolymer of L-lactide, $\varepsilon$-caprolactone and nano-hydroxyapatite for bone tissue engineering. Mater. Sci. Eng. C 2014, 42, 199-210.

538. Wang, R.Z.; Cui, F.Z.; Lu, H.B.; Wen, H.B.; Ma, C.L.; Li, H.D. Synthesis of nanophase hydroxyapatite/collagen composite. J. Mater. Sci. Lett. 1995, 14, 490-492.

539. Kikuchi, M.; Itoh, S.; Ichinose, S.; Shinomiya, K.; Tanaka, J. Self-organization mechanism in a bone-like hydroxyapatite/collagen nanocomposite synthesized in vitro and its biological reaction in vivo. Biomaterials 2001, 22, 1705-1711.

540. Kikuchi, M.; Matsumoto, H.N.; Yamada, T.; Koyama, Y.; Takakuda, K.; Tanaka, J. Glutaraldehyde cross-linked hydroxyapatite/collagen self-organized nanocomposites. Biomaterials 2004, 25, 63-69.

541. Lynn, A.K.; Nakamura, T.; Patel, N.; Porter, A.E.; Renouf, A.C.; Laity, P.R.; Best, S.M.; Cameron, R.E.; Shimizu, Y.; Bonfield, W. Composition-controlled nanocomposites of apatite and collagen incorporating silicon as osseopromotive agent. J. Biomed. Mater. Res. A 2005, 74, 447-453.

542. Chang, M.C.; Tanaka, J. FTIR study for hydroxyapatite/collagen nanocomposite cross-linked by glutaraldehyde. Biomaterials 2002, 23, 4811-4818.

543. Chang, M.C.; Tanaka, J. XPS study for the microstructure development of hydroxyapatitecollagen nanocomposites cross-linked using glutaraldehyde. Biomaterials 2002, 23, 3879-3885.

544. Thomas, V.; Dean, D.R.; Jose, M.V.; Mathew, B.; Chowdhury, S.; Vohra. Y.K. Nanostructured biocomposite scaffolds based on collagen coelectrospun with nanohydroxyapatite. Biomacromolecules 2007, 8, 631-637.

545. Fukui, N.; Sato, T.; Kuboki, Y.; Aoki, H. Bone tissue reaction of nano-hydroxyapatite/collagen composite at the early stage of implantation. Biomed. Mater. Eng. 2008, 18, 25-33.

546. Kim, T.G.; Park, S.H.; Chung, H.J.; Yang, D.Y.; Park, T.G. Microstructured scaffold coated with hydroxyapatite/collagen nanocomposite multilayer for enhanced osteogenic induction of human mesenchymal stem cells. J. Mater. Chem. 2010, 20, 8927-8933.

547. Ebrahimi, M.; Pripatnanont, P.; Suttapreyasri, S.; Monmaturapoj, N. In vitro biocompatibility analysis of novel nano-biphasic calcium phosphate scaffolds in different composition ratios. $J$. Biomed. Mater. Res. B Appl. Biomater. 2014, 102, 52-61.

548. Chen, L.; Hu, J.; Ran, J.; Shen, X.; Tong, H. Synthesis and cytocompatibility of collagen/hydroxyapatite nanocomposite scaffold for bone tissue engineering. Polym. Compos. 2015, doi:10.1002/pc.23157. 
549. Liao, S.S.; Cui, F.Z.; Zhu, Y. Osteoblasts adherence and migration through three-dimensional porous mineralized collagen based composite: nHAC/PLA. J. Bioact. Compat. Polym. 2004, 19, $117-130$.

550. Liao, S.S.; Cui, F.Z.; Zhang, W.; Feng, Q.L. Hierarchically biomimetic bone scaffold materials: nano-HA/collagen/PLA composite. J. Biomed. Mater. Res. B Appl. Biomater. 2004, 69, 158-165.

551. Liao, S.S.; Cui, F.Z. In vitro and in vivo degradation of the mineralized collagen based composite scaffold: nanohydroxyapatite/collagen/poly(L-lactide). Tissue Eng. 2004, 10, 73-80.

552. Liao, S.S.; Wang, W.; Uo, M.; Ohkawa, S.; Akasaka, T.; Tamura, K.; Cui, F.Z.; Watari, F. A three-layered nano-carbonated hydroxyapatite/collagen/PLGA composite membrane for guided tissue regeneration. Biomaterials 2005, 26, 7564-7571.

553. Zhang, C.; Hu, Y.Y.; Cui, F.Z.; Zhang, S.M.; Ruan, D.K. A study on a tissue-engineered bone using rhBMP-2 induced periosteal cells with a porous nano-hydroxyapatite/collagen/poly(L-lactic acid) scaffold. Biomed. Mater. 2006, 1, 56-62.

554. Liao, S.; Watari, F.; Zhu, Y.; Uo, M.; Akasaka, T.; Wang, W.; Xu, G.; Cui, F.Z. The degradation of the three layered nano-carbonated hydroxyapatite/collagen/PLGA composite membrane in vitro. Dent. Mater. 2007, 23, 1120-1128.

555. Degirmenbasi, N.; Kalyon, D.M.; Birinci, E. Biocomposites of nanohydroxyapatite with collagen and poly(vinyl alcohol). Colloids Surf. B 2006, 48, 42-49.

556. Zhang, S.M.; Cui, F.Z.; Liao, S.S.; Zhu, Y.; Han, L. Synthesis and biocompatibility of porous nanohydroxyapatite/collagen/alginate composite. J. Mater. Sci. Mater. Med. 2003, 14, 641-645.

557. Sotome, S.; Uemura, T.; Kikuchi, M.; Chen, J.; Itoh, S.; Tanaka, J.; Tateishi, T.; Shinomiya, K. Synthesis and in vivo evaluation of a novel hydroxyapatite/collagen-alginate as a bone filler and a drug delivery carrier of bone morphogenetic protein. Mater. Sci. Eng. C 2004, 24, 341-347.

558. Pandi, K.; Viswanathan, N. Synthesis of alginate bioencapsulated nano-hydroxyapatite composite for selective fluoride sorption. Carbohyd. Polym. 2014, 112, 662-667.

559. Chang, M.C.; Ikoma, T.; Tanaka, J. Cross-linkage of hydroxyapatite/gelatin nanocomposite using EGDE. J. Mater. Sci. 2004, 39, 5547-5550.

560. Teng, S.; Shi, J.; Peng, B.; Chen, L. The effect of alginate addition on the structure and morphology of hydroxyapatite/gelatin nanocomposites. Compos. Sci. Technol. 2006, 66, 1532-1538.

561. Chang, M.C.; Ko, C.C.; Douglas, W.H. Preparation of hydroxyapatite-gelatin nanocomposite. Biomaterials 2003, 24, 2853-2862.

562. Mobini, S.; Javadpour, J.; Hosseinalipour, M.; Ghazi-Khansari, M.; Khavandi, A.; Rezaie, H.R. Synthesis and characterisation of gelatin-nano hydroxyapatite composite scaffolds for bone tissue engineering. Adv. Appl. Ceram. 2008, 107, 4-8.

563. Mostafa, A.A.; Ibrahim, D.M.; Korowash, S.I.; Fahim, F.; Oudadesse, H. Nano-hybrid-composite scaffolds from substituted apatite/gelatin. Key Eng. Mater. 2014, 587, 233-238.

564. Wang, H.; Bongio, M.; Farbod, K.; Nijhuis, A.W.; van den Beucken, J.; Boerman, O.C.; van Hest, J.C.; Li, Y.; Jansen, J.A.; Leeuwenburgh, S.C. Development of injectable organic/inorganic colloidal composite gels made of self-assembling gelatin nanospheres and calcium phosphate nanocrystals. Acta Biomater. 2014, 10, 508-519. 
565. Lewandrowski, K.U.; Bondre, S.P.; Wise, D.L.; Trantolo, D.J. Enhanced bioactivity of a poly(propylene fumarate) bone graft substitute by augmentation with nano-hydroxyapatite. Biomed. Mater. Eng. 2003, 13, 115-124.

566. Jayabalan, M.; Shalumon, K.T.; Mitha, M.K.; Ganesan, K.; Epple, M. Effect of hydroxyapatite on the biodegradation and biomechanical stability of polyester nanocomposites for orthopaedic applications. Acta Biomater. 2010, 6, 763-775.

567. Jayabalan, M.; Shalumon, K.T.; Mitha, M.K.; Ganesan, K.; Epple, M. The effect of radiation processing and filler morphology on the biomechanical stability of a thermoset polyester composite. Biomed. Mater. 2010, 5, doi:10.1088/1748-6041/5/2/025009.

568. Wei, J.; Li, Y.; Chen, W.; Zuo, Y. A study on nano-composite of hydroxyapatite and polyamide. J. Mater. Sci. 2003, 38, 3303-3306.

569. Wang, H.; Li, Y.; Zuo, Y.; Li, J.; Ma, S.; Cheng, L. Biocompatibility and osteogenesis of biomimetic nano-hydroxyapatite/polyamide composite scaffolds for bone tissue engineering. Biomaterials 2007, 28, 3338-3348.

570. Sender, C.; Dantras, E.; Dantras-Laffont, L.; Lacoste, M.H.; Dandurand, J.; Mauzac, M.; Lacout, J.L.; Lavergne, C.; Demont, P.; Bernes, A.; et al. Dynamic mechanical properties of a biomimetic hydroxyapatite/polyamide 6,9 nanocomposite. J. Biomed. Mater. Res. B Appl. Biomater. 2007, $83,628-635$.

571. Zhang, X.; Li, Y.; Zuo, Y.; Lv, G.Y.; Mu, Y.H.; Li, H. Morphology, hydrogen-bonding and crystallinity of nano-hydroxyapatite/polyamide 66 biocomposites. Composites $A$ 2007, 38, 843-848.

572. Zhang, L.; Li, Y.; Wang, X.; Wei, J.; Peng, X. Studies on the porous scaffold made of the nanoHA/PA66 composite. J. Mater. Sci. 2005, 40, 107-110.

573. Zhang, X.; Li, Y.; Lv, G.Y.; Zuo, Y.; Mu, Y.H.; Lan, W. The study on interaction mechanism between n-HA and PA66 in n-HA/PA66 biocomposites. Funct. Mater. 2005, 36, 896-899.

574. Menon, D.; Anand, K.A.; Anitha, V.C.; Nair, S. Hydroxyapatite-reinforced polyamide 6,6 nanocomposites through melt compounding. Int. J. Polym. Mater. 2010, 59, 498-509.

575. Hu, G.; Wang, H.; Yao, X.; Bi, D.; Zhu, G.; Tang, S.; Wei, J.; Yang, L.; Tong, P.; Xiao, L. Development of nanofluorapatite polymer-based composite for bioactive orthopedic implants and prostheses. Int. J. Nanomed. 2014, 9, 3875-3884.

576. Yusong, P.; Dangsheng, X.; Xiaolin, C. Mechanical properties of nanohydroxyapatite reinforced poly(vinyl alcohol) gel composites as biomaterial. J. Mater. Sci. 2007, 42, 5129-5134.

577. Xu, F.; Li, Y.; Wang, X.; Wei, J.; Yang, A. Preparation and characterization of nanohydroxyapatite/poly(vinyl alcohol) hydrogel biocomposite. J. Mater. Sci. 2004, 39, 5669-5672.

578. Wang, H.S.; Wang, G.X.; Pan, Q.X. Electrochemical study of the interactions of DNA with redox-active molecules based on the immobilization of dsDNA on the sol-gel derived nano porous hydroxyapatite-polyvinyl alcohol hybrid material coating. Electroanalysis 2005, 17, 1854-1860.

579. Pramanik, N.; Mohapatra, S.; Pramanik, P.; Bhargava, P. Processing and properties of nanohydroxyapatite (n-HAp)/poly(ethylene-co-acrylic acid) (EAA) composite using a phosphonic acid coupling agent for orthopedic applications. J. Am. Ceram. Soc. 2007, 90, 369-375. 
580. Pramanik, N.; Bhargava, P.; Alam, S.; Pramanik, P. Processing and properties of nano- and macro-hydroxyapatite/poly(ethylene-co-acrylic acid) composites. Polym. Compos. 2006, 27, 633-641.

581. Zhang, Y.F.; Cheng, X.R.; Chen, Y.; Shi, B.; Chen, X.H.; Xu, D.X.; Ke, J. Three-dimensional nanohydroxyapatite/chitosan scaffolds as potential tissue engineered periodontal tissue. $J$. Biomater. Appl. 2007, 21, 333-349.

582. Lu, X.Y.; Wang, X.H.; Qu, S.X.; Weng, J. Preparation of nano-hydroxyapatite/chitosan hybrids. J. Inorg. Mater. 2008, 23, 332-336.

583. Chen, J.D.; Wang, Y.; Chen, X. In situ fabrication of nano-hydroxyapatite in a macroporous chitosan scaffold for tissue engineering. J. Biomater. Sci. Polym. Edn. 2009, 20, 1555-1565.

584. Thein-Han, W.W.; Misra, R.D.K. Biomimetic chitosan-nanohydroxyapatite composite scaffolds for bone tissue engineering. Acta Biomater. 2009, 5, 1182-1197.

585. Chen, J.; Nan, K.; Yin, S.; Wang, Y.; Wu, T.; Zhang, Q. Characterization and biocompatibility of nanohybrid scaffold prepared via in situ crystallization of hydroxyapatite in chitosan matrix. Colloids Surf. B 2010, 81, 640-647.

586. Ma, X.; Wang, Y.; Guo H.; Wang, J. Nano-hydroxyapatite/chitosan sponge-like biocomposite for repairing of rat calvarial critical-sized bone defect. J. Bioact. Compat. Polym. 2011, 26, 335-346.

587. Reves, B.T.; Jennings, J.A.; Bumgardner, J.D.; Haggard, W.O. Preparation and functional assessment of composite chitosan-nano-hydroxyapatite scaffolds for bone regeneration. J. Funct. Biomater. 2012, 3, 114-130.

588. Qu, Y.; Ao, D.; Wang, P.; Wang, Y.; Kong, X.; Man, Y. Chitosan/nano-hydroxyapatite composite electret membranes enhance cell proliferation and osteoblastic expression in vitro. J. Bioact. Compat. Polym. 2014, 29, 3-14.

589. Roy, P.; Sailaja, R.R.N. Chitosan-nanohydroxyapatite composites: mechanical, thermal and bio-compatibility studies. Int. J. Biol. Macromol. 2015, 73, 170-181.

590. Lu, Y.; Zhu, A.; Wang, W.; Shi, H. New bioactive hybrid material of nano-hydroxyapatite based on N-carboxyethylchitosan for bone tissue engineering. Appl. Surf. Sci. 2010, 256, 7228-7233.

591. Zhou, G.; Li, Y.; Zhang, L.; Li, H.; Wang, M.; Cheng, L.; Wang, Y.; Wang, H.; Shi, P. The study of tri-phasic interactions in nano-hydroxyapatite/konjac glucomannan/chitosan composite. $J$. Mater. Sci. 2007, 42, 2591-2597.

592. Mohamed, K.R.; El-Rashidy, Z.M.; Salama, A.A. Preparation and characterization of nano hydroxyapatite/polymeric composites materials. Part I. Mater. Chem. Phys. 2011, 130, 561-568.

593. Bueno, V.B.; Bentini, R.; Catalani, L.H.; Barbosa, L.R.S.; Petri, D.F.S. Synthesis and characterization of xanthan-hydroxyapatite nanocomposites for cellular uptake. Mater. Sci. Eng. C 2014, 37, $195-230$.

594. Huang, J.; Lin, Y.W.; Fu, X.W.; Best, S.M.; Brooks, R.A.; Rushton, N.; Bonfield, W. Development of nano-sized hydroxyapatite reinforced composites for tissue engineering scaffolds. J. Mater. Sci. Mater. Med. 2007, 18, 2151-2157.

595. Lee, H.J.; Choi, H.W.; Kim, K.J.; Lee, S.C. Modification of hydroxyapatite nanosurfaces for enhanced colloidal stability and improved interfacial adhesion in nanocomposites. Chem. Mater. 2006, 18, 5111-5118. 
596. Lee, H.J.; Kim, S.E.; Choi, H.W.; Kim, C.W.; Kim, K.J.; Lee, S.C. The effect of surface-modified

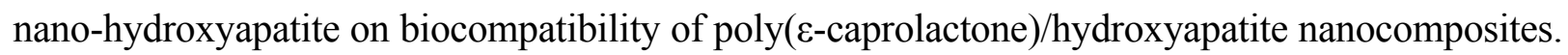
Eur. Polym. J. 2007, 43, 1602-1608.

597. Hao, J.Y.; Liu, Y.; Zhou, S.; Li, Z.; Deng, X. Investigation of nanocomposites based on semi-interpenetrating network of [L-poly ( $\varepsilon$-caprolactone)]/[net-poly ( $\varepsilon$-caprolactone)] and hydroxyapatite nanocrystals. Biomaterials 2003, 24, 1531-1539.

598. Boissard, C.I.R.; Bourban, P.E.; Tami, A.E.; Alini, M.; Eglin, D. Nanohydroxyapatite/poly(ester urethane) scaffold for bone tissue engineering. Acta Biomater. 2009, 5, 3316-3327.

599. Grande, C.J.; Torres, F.G.; Gomez, C.M.; Bañó, M.C. Nanocomposites of bacterial cellulose/hydroxyapatite for biomedical applications. Acta Biomater. 2009, 5, 1605-1615.

600. Zadegan, S.; Hossainalipour, M.; Ghassai, H.; Rezaie, H.R.; Naimi-Jamal, M.R. Synthesis of cellulose-nanohydroxyapatite composite in 1-n-butyl-3-methylimidazolium chloride. Ceram. Int. 2010, 36, 2375-2381.

601. Jia, N.; Li, S.M.; Zhu, J.F.; Ma, M.G.; Xu, F.; Wang, B.; Sun, R.C. Microwave-assisted synthesis and characterization of cellulose-carbonated hydroxyapatite nanocomposites in $\mathrm{NaOH}$-urea aqueous solution. Mater. Lett. 2010, 64, 2223-2225.

602. Pang, P.; Li, W.; Liu, Y. Effect of ball milling process on the microstructure of titaniumnanohydroxyapatite composite powder. Rare Metals 2007, 26, 118-123.

603. Li, W.; Pang, P.; Liu, Y. Microstructure and phase composition of Ti-based biocomposites with different contents of nano-hydroxyapatite. Trans. Nonferrous Metals Soc. China 2007, 17, S1148-S1151.

604. Niespodziana, K.; Jurczyk, K.; Jakubowicz, J.; Jurczyk, M. Fabrication and properties of titanium-hydroxyapatite nanocomposites. Mater. Chem. Phys. 2010, 123, 160-165.

605. Brook, I.; Freeman, C.; Grubb, S.; Cummins, N.; Curran, D.; Reidy, C.; Hampshire, S.; Towler, M. Biological evaluation of nano-hydroxyapatite-zirconia $\left(\mathrm{HA}-\mathrm{ZrO}_{2}\right)$ composites and strontiumhydroxyapatite (Sr-HA) for load-bearing applications. J. Biomater. Appl. 2012, 27, 291-298.

606. Chaudhry, A.A.; Yan, H.; Viola, G.; Reece, M.J.; Knowles, J.C.; Gong, K.; Rehman, I.; Darr, J.A. Phase stability and rapid consolidation of hydroxyapatite-zirconia nano-coprecipitates made using continuous hydrothermal flow synthesis. J. Biomater. Appl. 2012, 27, 79-90.

607. Gain, A.K.; Zhang, L.; Liu, W. Microstructure and material properties of porous hydroxyapatitezirconia nanocomposites using polymethyl methacrylate powders. Mater. Des. 2015, 67, 136-144.

608. Cicuéndez, M.; Portolés, M.T.; Izquierdo-Barba, I.; Vallet-Regí, M. New nanocomposite system with nanocrystalline apatite embedded into mesoporous bioactive glass. Chem. Mater. 2012, 24 , 1100-1106.

609. Govindan, R.; Girija, E.K. Drug loaded phosphate glass/hydroxyapatite nanocomposite for orthopedic applications. J. Mater. Chem. B 2014, 2, 5468-5477.

610. Akhavan, A.; Sheikh, N.; Khoylou, F.; Naimian, F.; Ataeivarjovi, E. Synthesis of antimicrobial silver/hydroxyapatite nanocomposite by gamma irradiation. Radiat. Phys. Chem. 2014, 98, $46-50$. 
611. Bahrami, M.; Fathi, M.H.; Ahmadian, M. The effect of nanobioceramic reinforcement on mechanical and biological properties of Co-base alloy/hydroxyapatite nanocomposite. Mater. Sci. Eng. C 2015, 48, 572-578.

612. Yan, Y.; Li, Y.; Zheng, Y.; Yi, Z.; Wei, J.; Xia, C.; Chen, Y. Synthesis and properties of a copolymer of poly(1,4-phenylene sulfide)-poly(2,4-phenylene sulfide acid) and its nano-apatite reinforced composite. Eur. Polym. J. 2003, 39, 411-416.

613. Bhattacharyya, S.; Nair, L.S.; Singh, A.; Krogman, N.R.; Bender, J.; Greish, Y.E.; Brown, P.W.; Allcock, H.R.; Laurencin, C.T. Development of biodegradable polyphosphazene-nanohydroxyapatite composite nanofibers via electrospinning. MRS Symp. Proc. 2005, 845, 91-96.

614. Zuo, Y.; Li, Y.; Wei, J.; Han, J.; Xu, F. The preparation and characterization of n-HA/PA series biomedical composite. Funct. Mater. 2004, 35, 513-516.

615. Zhou, G.; Li, Y.; Zhang, L.; Zuo, Y.; Jansen, J.A. Preparation and characterization of nanohydroxyapatite/chitosan/konjac glucomannan composite. J. Biomed. Mater. Res. A 2007, 83, 931-939.

616. Daniel-da-Silva, A.L.; Lopes, A.B.; Gil, A.M.; Correia, R.N. Synthesis and characterization of porous $\kappa$-carrageenan/calcium phosphate nanocomposite scaffolds. J. Mater. Sci. 2007, 42, 8581-8591.

617. Yang, K.; Wang, C.; Wei, J. A study on biocomposite of nano apatite/poly (1,4-phenylenesulfide)-poly (2,4-phenylene sulfide acid). Composites B 2007, 38, 306-310.

618. Jiang, L.; Li, Y.; Zhang, L.; Liao, J. Preparation and properties of a novel bone repair composite: Nano-hydroxyapatite/chitosan/carboxymethyl cellulose. J. Mater. Sci. Mater. Med. 2008, 19, 981-987.

619. Liu, L.; Liu, J.; Wang, M.; Min, S.; Cai, Y.; Zhu, L.; Yao, J. Preparation and characterization of nano-hydroxyapatite/silk fibroin porous scaffolds. J. Biomater. Sci. Polym. Edn. 2008, 19, 325-338.

620. Ren, Y.J.; Sun, X.D.; Cui, F.Z.; Wei, Y.T.; Cheng, Z.J.; Kong, X.D. Preparation and characterization of antheraea pernyi silk fibroin based nanohydroxyapatite composites. J. Bioact. Compat. Polym. 2007, 22, 465-474.

621. Wei, J.; Li, Y.; Lau, K.T. Preparation and characterization of a nano apatite/polyamide 6 bioactive composite. Composites B 2007, 38, 301-305.

622. Sundaraseelan, J.; Sastry, T.P. Fabrication of a biomimetic compound containing nano hydroxyapatite-Demineralised bone matrix. J. Biomed. Nanotechnol. 2007, 3, 401-405.

623. Hesaraki, S.; Ebadzadeh, T.; Ahmadzadeh-Asl, S. Nanosilicon carbide/hydroxyapatite nanocomposites: Structural, mechanical and in vitro cellular properties. J. Mater. Sci. Mater. Med. 2010, 21, 2141-2149.

624. Niu, Y.; Cao, L.; Wei, J.; Ma, Y.; Song, S.; Weng, W.; Li, H.; Liu, C.; Su, J. Development of a bioactive composite of nano fluorapatite and poly(butylene succinate) for bone tissue regeneration. J. Mater. Chem. B 2014, 2, 1174-1181.

625. Li, H.; Yang, L.; Dong, X.; Gu, Y.; Lv, G.; Yan, Y. Composite scaffolds of nano calcium deficient hydroxyapatite/multi-(amino acid) copolymer for bone tissue regeneration. J. Mater. Sci. Mater. Med. 2014, 25, 1257-1265. 
626. Pourdanesh, F.; Jebali, A.; Hekmatimoghaddam, S.; Allaveisie, A. In vitro and in vivo evaluation of a new nanocomposite, containing high density polyethylene, tricalcium phosphate, hydroxyapatite, and magnesium oxide nanoparticles. Mater. Sci. Eng. C 2014, 40, 382-388.

627. Pistone, A.; Iannazzo, D.; Panseri, S.; Montesi, M.; Tampieri, A.; Galvagno, S. Hydroxyapatitemagnetite-MWCNT nanocomposite as a biocompatible multifunctional drug delivery system for bone tissue engineering. Nanotechnol. 2014, 25, doi:10.1088/0957-4484/25/42/425701.

628. Li, Z.; Mi, W.; Wang, H.; Su, Y.; He, C. Nano-hydroxyapatite/polyacrylamide composite hydrogels with high mechanical strengths and cell adhesion properties. Colloids Surf. B 2014, 123, 959-964.

629. Siqueira, I.A.W.B.; Oliveira, C.A.G.S.; Zanin, H.; Grinet, M.A.V.M.; Granato, A.E.C.; Porcionatto, M.A.; Marciano, F.R.; Lobo, A.O. Bioactivity behaviour of nano-hydroxyapatite/ freestanding aligned carbon nanotube oxide composite. J. Mater. Sci. Mater. Med. 2015, 26, $1-10$.

630. Gheisari, H.; Karamian, E.; Abdellahi, M. A novel hydroxyapatite - hardystonite nanocomposite ceramic. Ceram. Int. 2015, 41, 5967-5975.

631. Sekar, S.; Mandal, A.; Manikandan, R.; Sankar, S.; Sastry, T.P. Synthesis and characterization of synthetic and natural nano hydroxyapatite composites containing poloxamer coated demineralized bone matrix as bone graft material: a comparative study. Int. J. Polym. Mater. Polym. Biomater. 2015, 64, 534-540.

632. Zandi, M.; Mirzadeh, H.; Mayer, C.; Urch, H.; Eslaminejad, M.B.; Bagheri, F.; Mivehchi, H. Biocompatibility evaluation of nano-rod hydroxyapatite/gelatin coated with nano-HAp as a novel scaffold using mesenchymal stem cells. J. Biomed. Mater. Res. A 2010, 92, 1244-1255.

633. Sun, T.S.; Guan, K.; Shi, S.S.; Zhu, B.; Zheng, Y.J.; Cui, F.Z.; Zhang, W.; Liao, S.S. Effect of nano-hydroxyapatite/collagen composite and bone morphogenetic protein-2 on lumbar intertransverse fusion in rabbits. Chin. J. Traumatol. 2004, 7, 18-24.

634. Itoh, S.; Kikuehi, M.; Koyama, Y.; Takakuda, K.; Shinomiya, K.; Tanaka, J. Development of a hydroxyapatite/collagen nanocomposite as a medical device. Cell Transp. 2004, 13, 451-461.

635. Kester, M.; Heakal, Y.; Fox, T.; Sharma, A.; Robertson, G.P.; Morgan, T.T.; Altinoğlu, E.I.; Tabaković, A.; Parette, M.R.; Rouse, S.M.; et al. Calcium phosphate nanocomposite particles for in vitro imaging and encapsulated chemotherapeutic drug delivery to cancer cells. Nano Lett. 2008, $8,4116-4121$.

636. Wang, K.W.; Zhou, L.Z.; Sun, Y.; Wu, G.J.; Gu, H.C.; Duan, Y.R.; Chen, F.; Zhu, Y.J. Calcium phosphate/PLGA-mPEG hybrid porous nanospheres: A promising vector with ultrahigh gene loading and transfection efficiency. J. Mater. Chem. 2010, 20, 1161-1166.

637. Gelinsky, M.; Welzel, P.B.; Simon, P.; Bernhardt, A.; König, U. Porous three-dimensional scaffolds made of mineralized collagen: Preparation and properties of a biomimetic nanocomposite material for tissue engineering of bone. Chem. Eng. J. 2008, 137, 84-96.

638. Hu, Q.; Li, B.Q.; Wang, M.; Shen, J.C. Preparation and characterization of biodegradable chitosan/hydroxyapatite nanocomposite rods via in situ hybridization: a potential material as internal fixation of bone fracture. Biomaterials 2004, 25, 779-785.

639. Wei, G.; Ma, P.X. Structure and properties of nano-hydroxyapatite/polymer composite scaffolds for bone tissue engineering. Biomaterials 2004, 25, 4749-4757. 
640. Liou, S.C.; Chen, S.Y.; Liu, D.M. Synthesis and characterization of needlelike apatitic nanocomposite with controlled aspect ratios. Biomaterials 2003, 24, 3981-3988.

641. Liou, S.C.; Chen, S.Y.; Liu, D.M. Manipulation of nanoneedle and nanosphere apatite/poly(acrylic acid) nanocomposites. J. Biomed. Mater. Res. B Appl. Biomater. 2005, 73, $117-122$.

642. Huang, J.; Best, S.M.; Bonfield, W.; Brooks, R.A.; Rushton, N.; Jayasinghe, S.N.; Edirisinghe, M.J. In vitro assessment of the biological response to nano-size hydroxyapatite. J. Mater. Sci. Mater. Med. 2004, 15, 441-415.

643. Kong, L.; Gao, Y.; Cao, W.; Gong, Y.; Zhao, N.; Zhang, X. Preparation and characterization of nano-hydroxyapatite/chitosan composite scaffolds. J. Biomed. Mater. Res. A 2005, 75, 275-282.

644. Venkatesan, J.; Kim, S.K. Nano-hydroxyapatite composite biomaterials for bone tissue engineering-A review. J. Biomed. Nanotechnol. 2014, 10, 3124-3140.

645. Christenson, E.M.; Anseth, K.S.; van den Beucken, J.J.J.P.; Chan, C.K.; Ercan, B.; Jansen, J.A.; Laurencin, C.T.; Li, W.J.; Murugan, R.; Nair, L.S.; et al. Nanobiomaterial applications in orthopedics. J. Orthop. Res. 2007, 25, 11-22.

646. Gelse, K.; Pöschl, E.; Aigner, T. Collagens - structure, function, and biosynthesis. Adv. Drug Deliv. Rev. 2003, 55, 1531-1546.

647. Fratzl, P. Collagen: Structure and Mechanics; Springer: New York, NY, USA, 2010; p. 510.

648. Tsai, C.H.; Chou, M.Y.; Jonas, M.; Tien, Y.T.; Chi, E.Y. A composite graft material containing bone particles and collagen in osteoinduction in mouse. J. Biomed. Mater. Res. 2002, 63, 65-70.

649. Xie, J.; Baumann, M.J.; McCabe, L.R. Osteoblasts respond to hydroxyapatite surfaces with immediate changes in gene expression. J. Biomed. Mater. Res. A 2004, 71A, 108-117.

650. Tcacencu, I.; Wendel, M. Collagen-hydroxyapatite composite enhances regeneration of calvaria bone defects in young rats but postpones the regeneration of calvaria bone in aged rats. J. Mater. Sci. Mater. Med. 2008, 19, 2015-2021.

651. Yamauchi, K.; Goda, T.; Takeuchi, N.; Einaga, H.; Tanabe, T. Preparation of collagen/calcium phosphate multilayer sheet using enzymatic mineralization. Biomaterials 2004, 25, 5481-5489.

652. Liu, C. Collagen-Hydroxyapatite Composite Scaffolds for Tissue Engineering. In Hydroxyapatite (HAp) for Biomedical Applications; Mucalo, M.R., Ed.; Elsevier: Cambridge, UK, 2015; pp. 211-234.

653. Hellmich, C.; Ulm, F.J. Are mineralized tissues open crystal foams reinforced by crosslinked collagen?-Some energy arguments. J. Biomech. 2002, 35, 1199-1212.

654. Roveri, N.; Falini, G.; Sidoti, M.C.; Tampieri, A.; Landi, E.; Sandri, M.; Parma, B. Biologically inspired growth of hydroxyapatite nanocrystals inside self-assembled collagen fibers. Mater. Sci. Eng. C 2003, 23, 441-446.

655. Tampieri, A.; Celotti, G.; Landi, E. From biomimetic apatites to biologically inspired composites. Anal. Bioanal. Chem. 2005, 381, 568-576.

656. Tampieri, A.; Celotti, G.; Landi, E.; Sandri, M.; Roveri, N.; Falini, G. Biologically inspired synthesis of bone-like composite: self-assembled collagen fibers/hydroxyapatite nanocrystals. $J$. Biomed. Mater. Res. A 2003, 67, 618-625.

657. Clarke, K.I.; Graves, S.E.; Wong, A.T.C.; Triffit, J.T.; Francis, M.J.O.; Czernuszka, J.T. Investigation into the formation and mechanical properties of a bioactive material based on collagen and calcium phosphate. J. Mater. Sci. Mater. Med. 1993, 4, 107-110. 
658. Ten Huisen, K.S.; Martin, R.I.; Klimkiewicz, M.; Brown, P.W. Formation and properties of a synthetic bone composite: hydroxyapatite-collagen. J. Biomed. Mater. Res. 1995, 29, 803-810.

659. Ishikawa, H.; Koshino, T.; Takeuchi, R.; Saito, T. Effects of collagen gel mixed with hydroxyapatite power on interface between newly formed bone and grafted Achilles tendon in rabbit femoral bone tunnel. Biomaterials 2001, 22, 1689-1694.

660. Itoh, S.; Kikuchi, M.; Takakuda, K.; Koyama, Y.; Matsumoto, H.N.; Ichinose, S.; Tanaka, J.; Kawauchi, T.; Shinomiya, K. The biocompatibility and osteoconductive activity of a novel hydroxyapatite/collagen composite biomaterial and its function as a carrier of rhBMP-2. J. Biomed. Mater. Res. 2001, 54, 445-453.

661. Uskokovic, V.; Ignjatovic, N.; Petranovic, N. Synthesis and characterization of hydroxyapatitecollagen biocomposite materials. Mater. Sci. Forum 2002, 413, 269-274.

662. Yoon, B.H.; Kim, H.W.; Lee, S.H.; Bae, C.J.; Koh, Y.H.; Kong, Y.M.; Kim, H.E. Stability and cellular responses to fluorapatite-collagen composites. Biomaterials 2005, 26, 2957-2963.

663. Wahl, D.A.; Czernuszka, J.T. Collagen-hydroxyapatite composites for hard tissue repair. Eur. Cell Mater. 2006, 11, 43-56.

664. Sachlos, E.; Gotora, D.; Czernuszka, J.T. Collagen scaffolds reinforced with biomimetic composite nano-sized carbonate-substituted hydroxyapatite crystals and shaped by rapid prototyping to contain internal microchannels. Tissue Eng. 2006, 12, 2479-2487.

665. Teng, S.H.; Lee, E.J.; Park, C.S.; Choi, W.Y.; Shin, D.S.; Kim, H.E. Bioactive nanocomposite coatings of collagen/hydroxyapatite on titanium substrates. J. Mater. Sci. Mater. Med. 2008, 19, 2453-2461.

666. Song, J.H.; Kim, H.E.; Kim, H.W. Collagen-apatite nanocomposite membranes for guided bone regeneration. J. Biomed. Mater. Res. B Appl. Biomater. 2007, 83, 248-257.

667. Pek, Y.S.; Gao, S.; Arshad, M.S.M.; Leck, K.J.; Ying, J.Y. Porous collagen-apatite nanocomposite foams as bone regeneration scaffolds. Biomaterials 2008, 29, 4300-4305.

668. Zhao, H.; Huang, C.; Jin, H.; Cai, J. A novel route for collagen/hydroxyapatite preparation by enzymatic decomposition of urea. J. Compos. Mater. 2010, 44, 2127-2133.

669. Kozłowska, J.; Sionkowska, A. Effects of different crosslinking methods on the properties of collagen-calcium phosphate composite materials. Int. J. Biol. Macromol. 2015, 74, 397-403.

670. Banglmaier, R.F.; Sander, E.A.; VandeVord, P.J. Induction and quantification of collagen fiber alignment in a three-dimensional hydroxyapatite-collagen composite scaffold. Acta Biomater. 2015, 17, 26-35.

671. Banks, E.; Nakajima, S.; Shapiro, L.C.; Tilevitz, O.; Alonzo, J.R.; Chianelli, R.R. Fibrous apatite grown on modified collagen. Science 1977, 198, 1164-1166.

672. Hayashi, K.; Yabuki, T.; Tabuchi, K.; Fujii, T. Repair of experimental bone defect with a collagen block containing synthesized apatite. Arch. Orthop. Traum. Surg. 1982, 99, 265-269.

673. Mittelmeier, H.; Nizzard, M. Knochenregeneration mit industriell gefertigtem Collagen Apatit Implantat ("Collapat"). In Osteogenese und Knochenwachstum; Hackenbroch, M.H., Refior, H.J., Jäger, M.G., Eds.; Thieme: Stuttgart, Germany, 1982; pp. 194-197.

674. Serre, C.M.; Papillard, M.; Chavassieux, P.; Boivin, G. In vitro induction of a calcifying matrix by biomaterials constituted of collagen and/or hydroxyapatite: an ultrastructural comparison of three types of biomaterials. Biomaterials 1993, 14, 97-106. 
675. Scabbia, A.; Trombelli, L. A comparative study on the use of a HA/collagen/chondroitin sulphate biomaterial $\left(\right.$ Biostite $^{\circledR}$ ) and a bovine-derived HA xenograft $\left(\right.$ Bio-Oss $\left.^{\circledR}\right)$ in the treatment of deep intraosseous defects. J. Clin. Periodontol. 2004, 31, 348-355.

676. Yamasaki, Y.; Yoshida, Y.; Okazaki, M.; Shimazu, A.; Kubo, T.; Akagawa, Y.; Uchida, T. Action of $\mathrm{FGMgCO}_{3} \mathrm{Ap}$-collagen composite in promoting bone formation. Biomaterials $\mathbf{2 0 0 3}$, 24, 4913-4920.

677. Wang, X.; Grogan, S.P.; Rieser, F.; Winkelmann, V.; Maquet, V.; Berge, M.L.; Mainil-Varlet, P. Tissue engineering of biphasic cartilage constructs using various biodegradable scaffolds: An in vitro study. Biomaterials 2004, 25, 3681-3688.

678. Chang, M.C.; Ikoma, T.; Kikuchi, M.; Tanaka, J. The cross-linkage effect of hydroxyapatite/collagen nanocomposites on a self-organization phenomenon. J. Mater. Sci. Mater. Med. 2002, 13, 993-997.

679. Iijima, M.; Moriwaki, Y.; Kuboki, Y. Oriented growth of octacalcium phosphate on and inside the collagenous matrix in vitro. Connect. Tissue Res. 1996, 32, 519-524.

680. Miyamoto, Y.; Ishikawa, K.; Takechi, M.; Toh, T.; Yuasa, T.; Nagayama, M.; Suzuki, K. Basic properties of calcium phosphate cement containing atelocollagen in its liquid or powder phases. Biomaterials 1998, 19, 707-715.

681. Iijima, M.; Moriwaki, Y.; Kuboki, Y. In vitro crystal growth of octacalcium phosphate on type I collagen fiber. J. Cryst. Growth 1994, 137, 553-560.

682. Iijima, M.; Iijima, K.; Moriwaki, Y.; Kuboki, Y. Oriented growth of octacalcium phosphate crystals on type I collagen fibrils under physiological conditions. J. Cryst. Growth 1994, 140, 91-99.

683. Lawson, A.C.; Czernuszka, J.T. Collagen - calcium phosphate composites. Proc. Inst. Mech. Eng. H 1998, 212, 413-425.

684. Du, C.; Cui, F.Z.; Zhang, W.; Feng, Q.L.; Zhu, X.D.; de Groot, K. Formation of calcium phosphate/collagen composites through mineralization of collagen matrix. J. Biomed. Mater. Res. 2000, 50, 518-527.

685. Kamakura, S.; Sasaki, K.; Honda, Y.; Anada, T.; Suzuki, O. Octacalcium phosphate combined with collagen orthotopically enhances bone regeneration. J. Biomed. Mater. Res. B Appl. Biomater. 2006, 79, 210-217.

686. Kawai, T.; Anada, T.; Honda, Y.; Kamakura, S.; Matsui, K.; Matsui, A.; Sasaki, K.; Morimoto, S.; Echigo, S, Suzuki, O. Synthetic octacalcium phosphate augments bone regeneration correlated with its content in collagen scaffold. Tissue Eng. A 2009, 15, 23-32.

687. Masuda, T.; Kawai, T.; Anada, T.; Kamakura, S.; Suzuki, O. Quality of regenerated bone enhanced by implantation of octacalcium phosphate-collagen composite. Tissue Eng. C 2010, 16, 471-478.

688. Kikuchi, M.; Ikoma, T.; Itoh, S.; Matsumoto, H.N.; Koyama, Y.; Takakuda, K.; Shinomiya, K.; Tanaka, J. Biomimetic synthesis of bone-like nanocomposites using the self-organization mechanism of hydroxyapatite and collagen. Compos. Sci. Technol. 2004, 64, 819-825.

689. Yunoki, S.; Ikoma, T.; Monkawal, A.; Ohtal, K.; Tanaka, J. Preparation and characterization of hydroxyapatite/collagen nanocomposite gel. J. Nanosci. Nanotechnol. 2007, 7, 818-821.

690. Li, X.; Chang, J. Preparation of bone-like apatite-collagen nanocomposites by a biomimetic process with phosphorylated collagen. J. Biomed. Mater. Res. A 2008, 85, 293-300. 
691. Sun, T.; Wang, M. Electrochemical deposition of apatite/collagen composite coating on NiTi shape memory alloy and coating properties. Mater. Res. Soc. Symp. Proc. 2010, 1239, 141-146.

692. Ficai, A.; Andronescu, E.; Voicu, G.; Ghitulica, C.; Vasile, B.S.; Ficai, D.; Trandafir, V. Selfassembled collagen/hydroxyapatite composite materials. Chem. Eng. J. 2010, 160, 794-800.

693. Jee, S.S.; Thula, T.T.; Gower, L.B. Development of bone-like composites via the polymer-induced liquid-precursor (PILP) process. Part 1: Influence of polymer molecular weight. Acta Biomater. 2010, 6, 3676-3686.

694. Miyazaki, T.; Kuramoto, A. Fabrication of composites from apatite and chemically synthesized collagen. Key Eng. Mater. 2013, 529-530, 426-429.

695. Andronescu, E.; Ficai, M.; Voicu, G.; Ficai, D.; Maganu, M.; Ficai, A. Synthesis and characterization of collagen/hydroxyapatite: magnetite composite material for bone cancer treatment. J. Mater. Sci. Mater. Med. 2010, 21, 2237-2242.

696. Ficai, M.; Andronescu, E.; Ficai, D.; Voicu, G.; Ficai, A. Synthesis and characterization of COLL-PVA/HA hybrid materials with stratified morphology. Colloids Surf. B 2010, 81, 614-619.

697. Inzana, J.A.; Olvera, D.; Fuller, S.M.; Kelly, J.P.; Graeve, O.A.; Schwarz, E.M.; Kates, S.L.; Awad, H.A. 3D printing of composite calcium phosphate and collagen scaffolds for bone regeneration. Biomaterials 2014, 35, 4026-4034.

698. Tamimi, F.; Kumarasami, B.; Doillon, C.; Gbureck, U.; Nihouannen, D.L.; Cabarcos, E.L.; Barralet, J.E. Brushite-collagen composites for bone regeneration. Acta Biomater. 2008, 4, 1315-1321.

699. Mai, R.; Reinstorf, A.; Pilling, E.; Hlawitschka, M.; Jung, R.; Gelinsky, M.; Schneider, M.; Loukota, R.; Pompe, W.; Eckelt, U.; et al. Histologic study of incorporation and resorption of a bone cement-collagen composite: An in vivo study in the minipig. Oral Surg. Oral Med. Oral Pathol. Oral Radiol. Endod. 2008, 105, e9-e14.

700. Moreau, J.L.; Weir, M.D.; Xu, H.H.K. Self-setting collagen-calcium phosphate bone cement: Mechanical and cellular properties. J. Biomed. Mater. Res. A 2009, 91, 605-613.

701. Liu, X.; Wang, X.M.; Chen, Z.; Cui, F.Z.; Liu, H.Y.; Mao, K.; Wang, Y. Injectable bone cement based on mineralized collagen. J. Biomed. Mater. Res. B Appl. Biomater. 2010, 94, 72-79.

702. Otsuka, M.; Nakagawa, H.; Ito, A.; Higuchi, W.I. Effect of geometrical structure on drug release rate of a three-dimensionally perforated porous apatite/collagen composite cement. J. Pharm. Sci. 2010, 99, 286-292.

703. Cui, F.Z.; Li, Y.; Ge, J. Self-assembly of mineralized collagen composites. Mater. Sci. Eng. $R$ 2007, 57, 1-27.

704. Hirota, K.; Nishihara, K.; Tanaka, H. Pressure sintering of apatite-collagen composite. Biomed. Mater. Eng. 1993, 3, 147-151.

705. Zahn, D.; Hochrein, O.; Kawska, A.; Brickmann, J.; Kniep, R. Towards an atomistic understanding of apatite-collagen biomaterials: linking molecular simulation studies of complex-, crystal- and composite-formation to experimental findings. J. Mater. Sci. 2007, 42, 8966-8973.

706. Silva, C.C.; Pinheiro, A.G.; Figueiro, S.D.; Goes, J.C.; Sasaki, J.M.; Miranda, M.A.R.; Sombra, A.S.B. Piezoelectric properties of collagen-nanocrystalline hydroxyapatite composites. J. Mater. Sci. 2002, 37, 2061-2070. 
707. Yunoki, S.; Ikoma, T.; Tsuchiya, A.; Monkawa, A.; Ohta, K.; Sotome, S.; Shinomiya, K.; Tanaka, J. Fabrication and mechanical and tissue ingrowth properties of unidirectionally porous hydroxyapatite/collagen composite. J. Biomed. Mater. Res. B Appl. Biomater. 2007, 80, 166-173.

708. Keeney, M.; Collin, E.; Pandit, A. Multi-channelled collagen-calcium phosphate scaffolds: their physical properties and human cell response. Tissue Eng. C 2009, 15, 265-273.

709. Chapman, M.W.; Bucholz, R.; Cornell, C. Treatment of acute fractures with a collagen-calcium phosphate graft material: A randomized clinical trial. J. Bone Joint Surg. (Am.) 1997, 79A, 495-502.

710. Rodrigues, C.V.M.; Serricella, P.; Linhares, A.B.R.; Guerdes, R.M.; Borojevic, R.; Rossi, M.A.; Duarte, M.E.L.; Farina, M. Characterization of a bovine collagen-hydroxyapatite composite scaffold for bone tissue engineering. Biomaterials 2003, 24, 4987-4997.

711. Miura, K.-I.; Anada, T.; Honda, Y.; Shiwaku, Y.; Kawai, T.; Echigo, S.; Takahashi, T.; Suzuki, O. Characterization and bioactivity of nano-submicro octacalcium phosphate/gelatin composite. Appl. Surf. Sci. 2013, 282, 138-145.

712. Kawai, T.; Echigo, S.; Matsui, K.; Tanuma, Y.; Takahashi, T.; Suzuki, O.; Kamakura, S. First clinical application of octacalcium phosphate collagen composite in human bone defect. Tissue Eng. A 2014, 20, 1336-1341.

713. Lickorish, D.; Ramshaw, J.A.M.; Werkmeister, J.A.; Glattauer, V.; Howlett, C.R. Development of a collagen-hydroxyapatite composite biomaterial via biomimetic process. J. Biomed. Mater. Res. A 2004, 68, 19-27.

714. Sionkowska, A.; Kozłowska, J. Characterization of collagen/hydroxyapatite composite sponges as a potential bone substitute. Int. J. Biol. Macromol. 2010, 47, 483-487.

715. Hsu, F.Y.; Chueh, S.C.; Wang, J.Y. Microspheres of hydroxyapatite/reconstituted collagen as supports for osteoblast cell growth. Biomaterials 1999, 20, 1931-1936.

716. Wu, T.J.; Huang, H.H.; Lan, C.W.; Lin, C.H.; Hsu, F.Y.; Wang, Y.J. Studies on the microspheres comprised of reconstituted collagen and hydroxyapatite. Biomaterials 2004, 25, 651-658.

717. Wei, Q.; Lu, J.; Wang, Q.; Fan, H.; Zhang, X. Novel synthesis strategy for composite hydrogel of collagen/hydroxyapatite-microsphere originating from conversion of $\mathrm{CaCO}_{3}$ templates. Nanotechnology 2015, 26, doi:doi:10.1088/0957-4484/26/11/115605.

718. Liao, S.S.; Watari, F.; Uo, M.; Ohkawa, S.; Tamura, K.; Wang, W.; Cui, F.Z. The preparation and characteristics of a carbonated hydroxyapatite/collagen composite at room temperature. $J$. Biomed. Mater. Res. B Appl. Biomater. 2005, 74, 817-821.

719. Yokoyama, A.; Gelinsky, M.; Kawasaki, T.; Kohgo, T.; König, U.; Pompe, W.; Watari, F. Biomimetic porous scaffolds with high elasticity made from mineralized collagen-An animal study. J. Biomed. Mater. Res. B Appl. Biomater. 2005, 75B, 464-472.

720. Zou, C.; Weng, W.; Deng, X.J.; Cheng, K.; Liu, X.; Du, P.; Shen, G.; Han, G. Preparation and characterization of porous $\beta$-tricalcium phosphate/collagen composites with an integrated structure. Biomaterials 2005, 26, 5276-5284.

721. Martins, V.C.A.; Goissis, G. Nonstoichiometric hydroxyapatite-anionic collagen composite as a support for the double sustained release of gentamicin and norfloxacin/ciprofloxacin. Artif. Organs 2000, 24, 224-230. 
722. Gotterbarm, T.; Richter, W.; Jung, M.; Berardi-Vilei, S.; Mainil-Varlet, P.; Yamashita, T.; Breusch, S.J. An in vivo study of a growth-factor enhanced, cell free, two-layered collagentricalcium phosphate in deep osteochondral defects. Biomaterials 2006, 27, 3387-3395.

723. Martins, V.C.; Goissis, G.; Ribeiro, A.C.; Marcantonio, E.; Jr.; Bet, M.R. The controlled release of antibiotic by hydroxyapatite: anionic collagen composites. Artif. Organs 1998, 22, 215-221.

724. Jayaraman, M.; Subramanian, M.V. Preparation and characterization of two new composites: Collagen-brushite and collagen-octacalcium phosphate. Medical Sci. Monitor 2002, 8, BR481-BR487.

725. Matsui, K.; Matsui, A.; Handa, T.; Kawai, T.; Suzuki, O.; Kamakura, S.; Echigo, S. Bone regeneration by octacalcium phosphate collagen composites in a dog alveolar cleft model. Int. J. Oral Maxillofac. Surg. 2010, 39, 1218-1225.

726. Iibuchi, S.; Matsui, K.; Kawai, T.; Sasaki, K.; Suzuki, O.; Kamakura, S.; Echigo, S. Octacalcium phosphate (OCP) collagen composites enhance bone healing in a dog tooth extraction socket model. Int. J. Oral Maxillofac. Surg. 2010, 39, 161-168.

727. Xia, Z.; Wei, M. Biomimetic fabrication of collagen-apatite scaffolds for bone tissue regeneration. J. Biomater. Tissue Eng. 2013, 3, 369-384.

728. Ikeda, H.; Yamaza, T.; Yoshinari, M.; Ohsaki, Y.; Ayukawa, Y.; Kido, M.A.; Inoue, T.; Shimono, M.; Koyano, K.; Tanaka, T. Ultrastructural and immunoelectron microscopic studies of the periimplant epithelium-implant (Ti-6Al-4V) interface of rat maxilla. J. Periodontol. 2000, 71, 961973.

729. Uchida, M.; Oyane, A.; Kim, H.M.; Kokubo, T.; Ito, A. Biomimetic coating of laminin-apatite composite on titanium metal and its excellent cell-adhesive properties. Adv. Mater. 2004, 16, 1071-1074.

730. Oyane, A.; Uchida, M.; Ito, A. Laminin-apatite composite coating to enhance cell adhesion to ethylene-vinyl alcohol copolymer. J. Biomed. Mater. Res. A 2005, 72A, 168-174.

731. Oyane, A.; Uchida, M.; Onuma, K.; Ito, A. Spontaneous growth of a laminin-apatite nano-composite in a metastable calcium phosphate solution. Biomaterials 2006, 27, 167-175.

732. Oyane, A.; Tsurushima, H.; Ito, A. Highly efficient gene transfer system using a laminin-DNA-apatite composite layer. J. Gene Med. 2010, 12, 194-206.

733. Oyane, A.; Wang, X.; Sogo, Y.; Ito, A.; Tsurushima, H. Calcium phosphate composite layers for surface-mediated gene transfer. Acta Biomater. 2012, 8, 2034-2046.

734. Yaylaoglu, M.B.; Korkusuz, P.; Ors, U.; Korkusuz, F.; Hasirci, V. Development of a calcium phosphate-gelatin composite as a bone substitute and its use in drug release. Biomaterials 1999, 20, 711-719.

735. Kim, H.W.; Knowles, J.C.; Kim, H.E. Porous scaffolds of gelatin-hydroxyapatite nanocomposites obtained by biomimetic approach: characterization and antibiotic drug release. J. Biomed. Mater. Res. B Appl. Biomater. 2005, 74, 686-698.

736. Hillig, W.B.; Choi, Y.; Murtha, S.; Natravali, N.; Ajayan, P. An open-pored gelatin/hydroxyapatite composite as a potential bone substitute. J. Mater. Sci. Mater. Med. 2008, 19, 11-17.

737. Chang, M.C.; Douglas, W.H.; Tanaka, J. Organic-inorganic interaction and the growth mechanism of hydroxyapatite crystals in gelatin matrices between 37 and $80^{\circ} \mathrm{C}$. J. Mater. Sci. Mater. Med. 2006, 17, 387-396. 
738. Chang, M.C.; Douglas, W.H. Cross-linkage of hydroxyapatite/gelatin nanocomposite using imide-based zero-length cross-linker. J. Mater. Sci. Mater. Med. 2007, 18, 2045-2051.

739. Liu, X.; Smith, L.A.; Hu, J.; Ma, P.X. Biomimetic nanofibrous gelatin/apatite composite scaffolds for bone tissue engineering. Biomaterials 2009, 30, 2252-2258.

740. Lin, H.R.; Yeh, Y.J. Porous alginate/hydroxyapatite composite scaffolds for bone tissue engineering: Preparation, characterization and in vitro studies. J. Biomed. Mater. Res. B Appl. Biomater. 2004, 71, 52-65.

741. Turco, G.; Marsich, E.; Bellomo, F.; Semeraro, S.; Donati, I.; Brun, F.; Grandolfo, M.; Accardo, A.; Paoletti, S. Alginate/hydroxyapatite biocomposite for bone ingrowth: a trabecular structure with high and isotropic connectivity. Biomacromolecules 2009, 10, 1575-1583.

742. Chae, T.; Yang, H.; Leung, V.; Ko, F.; Troczynski, T. Novel biomimetic hydroxyapatite/alginate nanocomposite fibrous scaffolds for bone tissue regeneration. J. Mater. Sci. Mater. Med. 2013, 24, 1885-1894.

743. Cuozzo, R.C.; da Leão, M.H.M.R.; de Gobbo, L.A.; da Rocha, D.N.; Ayad, N.M.E.; Trindade, W.; Costa, A.M.; da Silva, M.H.P. Zinc alginate-hydroxyapatite composite microspheres for bone repair. Ceram. Int. 2014, 40, 11369-11375.

744. Li, S.; Kan, B.; Zhao, K.; Ren, T.; Lin, B.; Wei, J.; Chen, T. Preparation of tricalcium phosphatecalcium alginate composite flat sheet membranes and their application for protein release. Polym. Composite 2015, doi:10.1002/pc.23098.

745. Won, J.E.; El-Fiqi, A.; Jegal, S.H.; Han, C.M.; Lee, E.J.; Knowles, J.C.; Kim, H.W. Gelatin-apatite bone mimetic co-precipitates incorporated within biopolymer matrix to improve mechanical and biological properties useful for hard tissue repair. J. Biomater. Appl. 2014, 28, 1213-1225.

746. Yamaguchi, I.; Tokuchi, K.; Fukuzaki, H.; Koyama, Y.; Takakuda, K.; Monma, H.; Tanaka, J. Preparation and microstructure analysis of chitosan/hydroxyapatite nanocomposites. J. Biomed. Mater. Res. 2001, 55, 20-27.

747. Zhang, Y.; Ni, M.; Zhang, M.Q.; Ratner, B. Calcium phosphate-Chitosan composite scaffolds for bone tissue engineering. Tissue Eng. 2003, 9, 337-345.

748. Tang, X.J.; Gui, L.; Lü, X.Y. Hard tissue compatibility of natural hydroxyapatite/chitosan composite. Biomed. Mater. 2008, 3, 044115.

749. Zhang, Y.; Venugopal, J.R.; El-Turki, A.; Ramakrishna, S.; Su, B.; Lim, C.T. Electrospun biomimetic nanocomposite nanofibers of hydroxyapatite/chitosan for bone tissue engineering. Biomaterials 2008, 29, 4314-4322.

750. Cai, X.; Tong, H.; Shen, X.; Chen, W.; Yan, J.; Hu, J. Preparation and characterization of homogeneous chitosan-polylactic acid/hydroxyapatite nanocomposite for bone tissue engineering and evaluation of its mechanical properties. Acta Biomater. 2010, 5, 2693-2703.

751. Ge, H.; Zhao, B.; Lai, Y.; Hu, X.; Zhang, D.; Hu, K. From crabshell to chitosan-hydroxyapatite composite material via a biomorphic mineralization synthesis method. J. Mater. Sci. Mater. Med. 2010, 21, 1781-1787.

752. Onoki, T.; Nakahira, A.; Tago, T.; Hasegawa, Y.; Kuno, T. Novel low temperature processing techniques for apatite ceramics and chitosan polymer composite bulk materials and its mechanical properties. Appl. Surf. Sci. 2012, 262, 263-266. 
753. Zugravu, M.V.; Smith, R.A.; Reves, B.T.; Jennings, J.A.; Cooper, J.O.; Haggard, W.O.; Bumgardner, J.D. Physical properties and in vitro evaluation of collagen-chitosan-calcium phosphate microparticle-based scaffolds for bone tissue regeneration. J. Biomater. Appl. 2013, 28, 566-579.

754. Fernández, T.; Olave, G.; Valencia, C.H.; Arce, S.; Quinn, J.M.W.; Thouas, G.A.; Chen, Q.Z. Effects of calcium phosphate/chitosan composite on bone healing in rats: calcium phosphate induces osteon formation. Tissue Eng. A 2014, 20, 1948-1960.

755. Tang, S.; Tian, B.; Guo, Y.J.; Zhu, Z.A.; Guo, Y.P. Chitosan/carbonated hydroxyapatite composite coatings: fabrication, structure and biocompatibility. Surf. Coat. Technol. 2014, 251, 210-216.

756. Leal, R.C.A.; Nascimento, I.V.S.R.; Fook, M.V.L.; Meira, R.C. Evaluation of the cytotoxic potential chitosan/hydroxyapatite biocomposites. Mater. Sci. Forum 2015, 805, 30-34.

757. Kucharska, M.; Walenko, K.; Lewandowska-Szumieł, M.; Brynk, T.; Jaroszewicz, J.; Ciach, T. Chitosan and composite microsphere-based scaffold for bone tissue engineering: evaluation of tricalcium phosphate content influence on physical and biological properties. J. Mater. Sci. Mater. Med. 2015, 26, 143:1-143:12.

758. Wan, A.C.A.; Khor, E.; Hastings, G.W. Preparation of a chitin-apatite composite by in situ precipitation onto porous chitin scaffolds. J. Biomed. Mater. Res. 1998, 41, 541-548.

759. Wan, A.C.A.; Khor, E.; Hastings, G.W. Hydroxyapatite modified chitin as potential hard tissue substitute material. J. Biomed. Mater. Res. 1997, 38, 235-241.

760. Geçer, A.; Yldz, N.; Erol, M.; Çalml, A. Synthesis of chitin calcium phosphate composite in different growth media. Polym. Composite 2008, 29, 84-91.

761. Dong, H.; Ye, J.D.; Wang, X.P.; Yang, J.J. Preparation of calcium phosphate cement tissue engineering scaffold reinforced with chitin fiber. J. Inorg. Mater. 2007, 22, 1007-1010.

762. Silva, S.S.; Duarte, A.R.C.; Oliveira, J.M.; Mano, J.F.; Reis, R.L. Alternative methodology for chitin-hydroxyapatite composites using ionic liquids and supercritical fluid technology. $J$. Bioact. Compat. Polym. 2013, 28, 481-491.

763. Wang, J.; Sun, Q.Z.; Gao, J.; Liu, D.M.; Meng, X.C.; Li, M.Q. Preparation and properties on silk fibers reinforced hydroxyapatite/chitosan composites. Adv. Mater. Res. 2010, 105-106, 557-560.

764. Zhang, Y.; Reddy, V.J.; Wong, S.Y.; Li, X.; Su, B.; Ramakrishna, S.; Lim, C.T. Enhanced biomineralization in osteoblasts on a novel electrospun biocomposite nanofibrous substrate of hydroxyapatite/collagen/chitosan. Tissue Eng. A 2010, 16, 1949-1960.

765. Kousalya, G.N.; Gandhi, R.M.; Sundaram, S.C.; Meenakshi, S. Synthesis of nano-hydroxyapatite chitin/chitosan hybrid biocomposites for the removal of Fe(III). Carbohydr. Polym. 2010, 82, 594-599.

766. Sundaram, C.S.; Viswanathan, N.; Meenakshi, S. Uptake of fluoride by nano-hydroxyapatite/chitosan, a bioinorganic composite. Bioresour. Technol. 2008, 99, 8226-8230.

767. Sundaram, C.S.; Viswanathan, N.; Meenakshi, S. Fluoride sorption by nano-hydroxyapatite/chitin composite. J. Hazard. Mater. 2009, 172, 147-151.

768. Wen, H.B.; de Wijn, J.R.; van Blitterswijk, C.A.; de Groot, K. Incorporation of bovine serum albumin in calcium phosphate coating on titanium. J. Biomed. Mater. Res. 1999, 46, 245-252. 
769. Liu, T.Y.; Chen, S.Y.; Liu, D.M.; Liou, S.C. On the study of BSA-loaded calcium-deficient hydroxyapatite nano-carriers for controlled drug delivery. J. Control. Release 2005, 107, 112-121.

770. Liu, Y.; Hunziker, E.; Randall, N.; de Groot, K.; Layrolle, P. Proteins incorporated into biomimetically prepared calcium phosphate coatings modulate their mechanical strength and dissolution rate. Biomaterials 2003, 24, 65-70.

771. Dorozhkin, S.V.; Dorozhkina, E.I. The influence of bovine serum albumin on the crystallization of calcium phosphates from a revised simulated body fluid. Colloids Surf. A 2003, 215, 191-199.

772. Fu, H.H.; Hu, Y.H.; McNelis, T.; Hollinger, J.O. A calcium phosphate-based gene delivery system. J. Biomed. Mater. Res. A 2005, 74A, 40-48.

773. Bisht, S.; Bhakta, G.; Mitra, S.; Maitra, A. pDNA loaded calcium phosphate nanoparticles: highly efficient non-viral vector for gene delivery. Int. J. Pharm. 2005, 288, 157-168.

774. Kakizawa, Y.; Miyata, K.; Furukawa, S.; Kataoka, K. Size-controlled formation of a calcium phosphate-based organic-inorganic hybrid vector for gene delivery using poly(ethylene glycol)block-poly(aspartic acid). Adv. Mater. 2004, 16, 699-702.

775. Singh, R.; Saxena, A.; Mozumdar, S. Calcium phosphate-DNA nanocomposites: morphological studies and their bile duct infusion for liver-directed gene therapy. Int. J. Appl. Ceram. Technol. 2008, 5, 1-10.

776. Oyane, A.; Araki, H.; Sogo, Y.; Ito, A.; Tsurushima, H. Coprecipitation of DNA and calcium phosphate using an infusion fluid mixture. Key Eng. Mater. 2013, 529-530, 465-470.

777. Sporysh, I.; Shynkaruk, E.; Lysko, O.; Shynkaruk, A.; Dubok, V.; Buzaneva, E.; Ritter, U.; Scharff, P. Biomimetic hydroxyapatite nanocrystals in composites with $\mathrm{C}_{60}$ and Au-DNA nanoparticles: IR-spectral study. Mater. Sci. Eng. B 2010, 169, 128-133.

778. Taguchi, T.; Kishida, A.; Akashi, M. Hydroxyapatite formation on/in poly(vinyl alcohol) hydrogel matrices using a novel alternate soaking process. Chem. Lett. 1998, 8, 711-712.

779. Tachaboonyakiat, W.; Serizawa, T.; Akashi, M. Hydroxyapatite formation on/in biodegradable chitosan hydrogels by an alternate soaking process. Polym. J. 2001, 33, 177-181.

780. Schnepp, Z.A.C.; Gonzalez-McQuire, R.; Mann, S. Hybrid biocomposites based on calcium phosphate mineralization of self-assembled supramolecular hydrogels. Adv. Mater. 2006, 18, 1869-1872.

781. Patel, M.; Patel, K.J.; Caccamese, J.F.; Coletti, D.P.; Sauk, J.J.; Fisher, J.P. Characterization of cyclic acetal hydroxyapatite nanocomposites for craniofacial tissue engineering. J. Biomed. Mater. Res. A 2010, 94, 408-418.

782. Bigi, A.; Boanini, E.; Gazzano, M.; Kojdecki, M.A.; Rubini, K. Microstructural investigation of hydroxyapatite-polyelectrolyte composites. J. Mater. Chem. 2004, 14, 274-279.

783. Bigi, A.; Boanini, E.; Gazzano, M.; Rubini, K.; Torricelli, P. Nanocrystalline hydroxyapatitepolyaspartate composites. Biomed. Mater. Eng. 2004, 14, 573-579.

784. Boanini, E.; Fini, M.; Gazzano, M.; Bigi, A. Hydroxyapatite nanocrystals modified with acidic amino acids. Eur. J. Inorg. Chem. 2006, 4821-4826.

785. Boanini, E.; Torricelli, P.; Gazzano, M.; Giardino, R.; Bigi, A. Nanocomposites of hydroxyapatite with aspartic acid and glutamic acid and their interaction with osteoblast-like cells. Biomaterials 2006, 27, 4428-4433. 
786. Ikawa, N.; Kimura, T.; Oumi, Y.; Sano, T. Amino acid containing amorphous calcium phosphates and the rapid transformation into apatite. J. Mater. Chem. 2009, 19, 4906-4913.

787. Sánchez-Salcedo, S.; Nieto, A.; Vallet-Regi, M. Hydroxyapatite/ $\beta$-tricalcium phosphate/agarose macroporous scaffolds for bone tissue engineering. Chem. Eng. J. 2005, 137, 62-71.

788. Román, J.; Cabañas, M.V.; Peña, J.; Doadrio, J.C.; Vallet-Regi, M. An optimized $\beta$-tricalcium phosphate and agarose scaffold fabrication technique. J. Biomed. Mater. Res. A 2008, 84, 99-107.

789. Alcaide, M.; Serrano, M.C.; Pagani, R.; Sánchez-Salcedo, S.; Nieto, A.; Vallet-Regí, M.; Portolés, M.T. L929 fibroblast and SAOS-2 osteoblast response to hydroxyapatite- $\beta$ TCP/ agarose biomaterial. J. Biomed. Mater. Res. A 2009, 89, 539-549.

790. Abiraman, S.; Varma, H.; Umashankar, P.; John, A. Fibrin sealant as an osteoinductive protein in a mouse model. Biomaterials 2002, 23, 3023-3031.

791. Wittkampf, A. Fibrin sealant as sealant for hydroxyapatite granules. J. Craniomaxillofac. Surg. 1989, 17, 179-181.

792. d'Arc, M.B.; Daculsi, G. Micro macroporous biphasic ceramics and fibrin sealant as a mouldable material for bone reconstruction in chronic otitis media surgery: A 15 years experience. J. Mater. Sci. Mater. Med. 2003, 14, 229-233.

793. le Nihouannen, D.; Guehennec, L.L.; Rouillon, T.; Pilet, P.; Bilban, M.; Layrolle, P.; Daculsi, G. Micro-architecture of calcium phosphate granules and fibrin glue composites for bone tissue engineering. Biomaterials 2006, 27, 2716-2722.

794. le Nihouannen D.; Saffarzadeh A.; Aguado E.; Goyenvalle E.; Gauthier O.; Moreau F.; Pilet P.; Spaethe R.; Daculsi, G.; Layrolle, P. Osteogenic properties of calcium phosphate ceramics and fibrin glue based composites. J. Mater. Sci. Mater. Med. 2007, 18, 225-235.

795. le Nihouannen, D.; Goyenvalle, E.; Aguado, E.; Pilet, P.; Bilban, M.; Daculsi, G.; Layrolle, P. Hybrid composites of calcium phosphate granules, fibrin glue, and bone marrow for skeletal repair. J. Biomed. Mater. Res. A 2007, 81A, 399-408.

796. Yoh, R.; Matsumoto, T.; Sasaki, J.I.; Sohmura, T. Biomimetic fabrication of fibrin/apatite composite material. J. Biomed. Mater. Res. A 2008, 87, 222-228.

797. Cui, G.; Li, J.; Lei, W.; Bi, L.; Tang, P.; Liang, Y.; Tao, S.; Wang, Y. The mechanical and biological properties of an injectable calcium phosphate cement-fibrin glue composite for bone regeneration. J. Biomed. Mater. Res. B Appl. Biomater. 2010, 92, 377-385.

798. Boanini, E.; Torricelli, P.; Gazzano, M.; Giardino, R.; Bigi, A. Alendronate-hydroxyapatite nanocomposites and their interaction with osteoclasts and osteoblast-like cells. Biomaterials 2008, 29, 790-796.

799. Wang, L.; Nemoto, R.; Senna, M. Microstructure and chemical states of hydroxyapatite/silk fibroin nanocomposites synthesized via a wet-mechanochemical route. J. Nanopart. Res. 2002, 4, 535-540.

800. Nemoto, R.; Wang, L.; Ikoma, T.; Tanaka, J.; Senna, M. Preferential alignment of hydroxyapatite crystallites in nanocomposites with chemically disintegrated silk fibroin. J. Nanopart. Res. 2004, 6, 259-265.

801. Wang, L.; Li, C.Z.; Senna, M. High-affinity integration of hydroxyapatite nanoparticles with chemically modified silk fibroin. J. Nanopart. Res. 2007, 9, 919-929. 
802. Li, L.; Wei, K.M.; Lin, F.; Kong, X.D.; Yao, J.M. Effect of silicon on the formation of silk fibroin/calcium phosphate composite. J. Mater. Sci. Mater. Med. 2008, 19, 577-582.

803. Fan, C.; Li, J.; Xu, G.; He, H.; Ye, X.; Chen, Y.; Sheng, X.; Fu, J.; He, D. Facile fabrication of nano-hydroxyapatite/silk fibroin composite via a simplified coprecipitation route. J. Mater. Sci. 2010, 45, 5814-5819.

804. Yan, L.P.; Silva-Correia, J.; Correia, C.; Caridade, S.G.; Fernandes, E.M.; Sousa, R.A.; Mano, J.F.; Oliveira, J.M.; Oliveira, A.L.; Reis, R.L. Bioactive macro/micro porous silk fibroin/nanosized calcium phosphate scaffolds with potential for bone-tissue-engineering applications. Nanomedicine 2013, 8, 359-378.

805. Salama, A.; Neumann, M.; Günter, C.; Taubert, A. Ionic liquid-assisted formation of cellulose/calcium phosphate hybrid materials. Beilstein J. Nanotechnol. 2014, 5, 1553-1568.

806. Wang, L.; Li, C.Z. Preparation and physicochemical properties of a novel hydroxyapatite/chitosan-silk fibroin composite. Carbohydr. Polym. 2007, 68, 740-745.

807. Oliveira, J.M.; Costa, S.A.; Leonor, I.B.; Malafaya, P.B.; Mano, J.F.; Reis, R.L. Novel hydroxyapatite/carboxymethylchitosan composite scaffolds prepared through an innovative "autocatalytic" electroless coprecipitation route. J. Biomed. Mater. Res. A 2009, 88, 470-480.

808. Sogo, Y.; Ito, A.; Matsuno, T.; Oyane, A.; Tamazawa, G.; Satoh, T.; Yamazaki, A.; Uchimura, E.; Ohno, T. Fibronectin-calcium phosphate composite layer on hydroxyapatite to enhance adhesion, cell spread and osteogenic differentiation of human mesenchymal stem cells in vitro. Biomed. Mater. 2007, 2, 116-123.

809. Rhee, S.H.; Suetsugu, Y.; Tanaka, J. Biomimetic configurational arrays of hydroxyapatite nanocrystals on bio-organics. Biomaterials 2001, 22, 2843-2847.

810. Cross, K.J.; Huq, N.L.; Palamara, J.E.; Perich, J.W.; Reynolds, E.C. Physicochemical characterization of casein phosphopeptide-amorphous calcium phosphate nanocomplexes. J. Biol. Chem. 2005, 280, 15362-15369.

811. Dimopoulou, M.; Ritzoulis, C.; Papastergiadis, E.S.; Panayiotou, C. Composite materials based on okra hydrocolloids and hydroxyapatite. Food Hydrocolloid 2014, 42, 348-354.

812. Nakata, R.; Tachibana, A.; Tanabe, T. Preparation of keratin hydrogel/hydroxyapatite composite and its evaluation as a controlled drug release carrier. Mater. Sci. Eng. C 2014, 41, 59-64.

813. Li, C.; Born, A.K.; Schweizer, T.; Zenobi-Wong, M.; Cerruti, M.; Mezzenga, R. Amyloidhydroxyapatite bone biomimetic composites. Adv. Mater. 2014, 26, 3207-3212.

814. Kolanthai, E.; Colon, V.S.D.; Sindu, P.A.; Chandra, V.S.; Karthikeyan, K.R.; Babu, M.S.; Sundaram, S.M.; Palanichamy, M.; Kalkura, S.N. Effect of solvent; enhancing the wettability and engineering the porous structure of a calcium phosphate/agarose composite for drug delivery. RSC Adv. 2015, 5, 18301-18311.

815. Jung, J.Y.; Hong, Y.J.; Choi, Y.S.; Jeong, S.; Lee, W.K. A new method for the preparation of

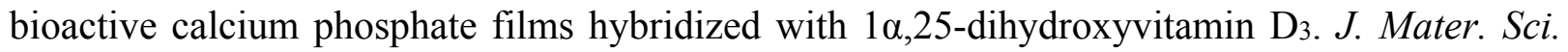
Mater. Med. 2010, 20, 2441-2453.

816. Killion, J.A.; Geever, L.M.; Devine, D.M.; Higginbotham, C.L. Fabrication and in vitro biological evaluation of photopolymerisable hydroxyapatite hydrogel composites for bone regeneration. J. Biomater. Appl. 2014, 28, 1274-1283. 
817. Shchukin, D.G.; Sukhorukov, G.B.; Möhwald, H. Biomimetic fabrication of nanoengineered hydroxyapatite/polyelectrolyte composite shell. Chem. Mater. 2003, 15, 3947-3950.

818. Jacoveila, P.F. Use of calcium hydroxylapatite $\left(\right.$ Radiesse $^{\circledR}$ ) for facial augmentation. Clin. Interv. Aging 2008, 3, 161-174.

819. Lizzul, P.F.; Narurkar, V.A. The role of calcium hydroxylapatite (Radiesse ${ }^{\circledR}$ ) in nonsurgical aesthetic rejuvenation. J. Drugs Dermatol. 2010, 9, 446-450.

820. Klesing, J.; Chernousova, S.; Kovtun, A.; Neumann, S.; Ruiz, L.; Gonzalez-Calbet, J.M.; ValletRegi, M.; Heumann, R.; Epple, M. An injectable paste of calcium phosphate nanorods, functionalized with nucleic acids, for cell transfection and gene silencing. J. Mater. Chem. 2010, 20, 6144-6148.

821. Thai, V.V.; Lee, B.T. Fabrication of calcium phosphate-calcium sulfate injectable bone substitute using hydroxy-propyl-methyl-cellulose and citric acid. J. Mater. Sci. Mater. Med. 2010, 21, 1867-1874.

822. Low, K.L.; Tan, S.H.; Zein, S.H.S.; Roether, J.A.; Mouriño, V.; Boccaccini, A.R. Calcium phosphate-based composites as injectable bone substitute materials. J. Biomed. Mater. Res. B Appl. Biomater. 2010, 94, 273-286.

823. D'Este, M.; Eglin, D. Hydrogels in calcium phosphate moldable and injectable bone substitutes: sticky excipients or advanced 3-D carriers? Acta Biomater. 2013, 9, 5421-5430.

824. Weiss, P.; Gauthier, O.; Bouler, J.M.; Grimandi, G.; Daculsi, G. Injectable bone substitute using a hydrophilic polymer. Bone 1999, 25, 67S-70S.

825. Daculsi, G.; Weiss, P.; Bouler, J.M.; Gauthier, O.; Millot, F.; Aguado, E. Biphasic calcium phosphate/hydrosoluble polymer composites: A new concept for bone and dental substitution biomaterials. Bone 1999, 25, 59S-61S.

826. Turczyn, R.; Weiss, P.; Lapkowski, M.; Daculsi, G. In situ self-hardening bioactive composite for bone and dental surgery. J. Biomater. Sci. Polym. Edn. 2000, 11, 217-223.

827. Bennett, S.; Connolly, K.; Lee, D.R.; Jiang, Y.; Buck, D.; Hollinger, J.O.; Gruskin, E.A. Initial biocompatibility studies of a novel degradable polymeric bone substitute that hardens in situ. Bone 1996, 19, 101S-107S.

828. Bongio, M.; van den Beucken, J.J.; Nejadnik, M.R.; Leeuwenburgh, S.C.; Kinard, L.A.; Kasper, F.K.; Mikos, A.G.; Jansen, J.A. Biomimetic modification of synthetic hydrogels by incorporation of adhesive peptides and calcium phosphate nanoparticles: in vitro evaluation of cell behavior. Eur. Cell. Mater. 2011, 22, 359-376.

829. Bongio, M.; van den Beucken, J.J.; Nejadnik, M.R.; Birgani, Z.T.; Habibovic, P.; Kinard, L.A.; Kasper, F.K.; Mikos, A.G.; Leeuwenburgh, S.C.; Jansen, J.A. Subcutaneous tissue response and osteogenic performance of calcium phosphate nanoparticle-enriched hydrogels in the tibial medullary cavity of guinea pigs. Acta Biomater. 2013, 9, 5464-5474.

830. Chernousova, S.; Klesing, J.; Soklakova, N.; Epple, M. A genetically active nano-calcium phosphate paste for bone substitution, encoding the formation of BMP-7 and VEGF-A. RSC Adv. 2013, 3, 11155-11161. 
831. Yu, B.; Zhang, Y.; Li, X.; Wang, Q.; Ouyang, Y.; Xia, Y.; Lin, B.; Li, S.; Fan, Y.; Chen, Y. The use of injectable chitosan/nanohydroxyapatite/collagen composites with bone marrow mesenchymal stem cells to promote ectopic bone formation in vivo. J. Nanomater. 2013, 2013, doi:10.1155/2013/506593.

832. Bodakhe, S.; Verma, S.; Garkhal, K.; Samal, S.K.; Sharma, S.S.; Kumar, N. Injectable photocrosslinkable nanocomposite based on poly(glycerol sebacate) fumarate and hydroxyapatite: Development, biocompatibility and bone regeneration in a rat calvarial bone defect model. Nanomedicine 2013, 8, 1777-1795.

833. Lin, G.; Cosimbescu, L.; Karin, N.J.; Tarasevich, B.J. Injectable and thermosensitive PLGA-g-PEG hydrogels containing hydroxyapatite: Preparation, characterization and in vitro release behavior. Biomed. Mater. 2012, 7, doi:10.1088/1748-6041/7/2/024107.

834. Nejadnik, M.R.; Yang, X.; Bongio, M.; Alghamdi, H.S.; van den Beucken, J.J.J.P.; Huysmans, M.C.; Jansen, J.A.; Hilborn, J.; Ossipov, D.; Leeuwenburgh, S.C.G. Self-healing hybrid nanocomposites consisting of bisphosphonated hyaluronan and calcium phosphate nanoparticles. Biomaterials 2014, 35, 6918-6929.

835. Munarin, F.; Petrini, P.; Gentilini, R.; Pillai, R.S.; Dirè, S.; Tanzi, M.C.; Sglavo, V.M. Microand nano-hydroxyapatite as active reinforcement for soft biocomposites. Int. J. Biol. Macromol. 2015, 72, 199-209.

836. Daculsi, G.; Rohanizadeh, R.; Weiss, P.; Bouler, J.M. Crystal polymer interaction with new injectable bone substitute: SEM and HrTEM study. J. Biomed. Mater. Res. 2000, 50, 1-7.

837. Grimande, G.; Weiss, P.; Millot, F.; Daculsi, G. In vitro evaluation of a new injectable calcium phosphate material. J. Biomed. Mater. Res. 1998, 39, 660-666.

838. Weiss, P.; Lapkowski, M.; LeGeros, R.Z.; Bouler, J.M.; Jean, A.; Daculsi, G. FTIR spectroscopic study of an organic/mineral composite for bone and dental substitute materials. J. Mater. Sci. Mater. Med. 1997, 8, 621-629.

839. Weiss, P.; Bohic, S.; Lapkowski, M.; Daculsi, G. Application of FTIR microspectroscopy to the study of an injectable composite for bone and dental surgery. J. Biomed. Mater. Res. 1998, 41, 167-170.

840. Schmitt, M.; Weiss, P.; Bourges, X.; del Valle, G.A.; Daculsi, G. Crystallization at the polymer/calcium-phosphate interface in a sterilized injectable bone substitute IBS. Biomaterials 2002, 23, 2789-2794.

841. Gauthier, O.; Müller, R.; von Stechow, D.; Lamy, B.; Weiss, P.; Bouler, J.M.; Aguado, E.; Daculsi, G. In vivo bone regeneration with injectable calcium phosphate biomaterial: A threedimensional micro-computed tomographic, biomechanical and SEM study. Biomaterials 2005, 26, 5444-5453.

842. Weiss, P.; Layrolle, P.; Clergeau, L.P.; Enckel, B.; Pilet, P.; Amouriq, Y.; Daculsi, G.; Giumelli, B. The safety and efficacy of an injectable bone substitute in dental sockets demonstrated in a human clinical trial. Biomaterials 2007, 28, 3295-3305.

843. Fatimi, A.; Tassin, J.F.; Axelos, M.A.V.; Weiss, P. The stability mechanisms of an injectable calcium phosphate ceramic suspension. J. Mater. Sci. Mater. Med. 2010, 21, 1799-1809.

844. Trojani, C.; Boukhechba, F.; Scimeca, J.C.; Vandenbos, F.; Michiels, J.F.; Daculsi, G.; Boileau, P.; Weiss, P.; Carle, G.F.; Rochet, N. Ectopic bone formation using an injectable biphasic calcium 
phosphate/Si-HPMC hydrogel composite loaded with undifferentiated bone marrow stromal cells. Biomaterials 2006, 27, 3256-3264.

845. Zhang, S.M.; Lü, G. Clinical application of compound injectable bone substitutes in bone injury repair. J. Clin. Rehabil. Tissue Eng. Res. 2009, 13, 10117-10120.

846. Daculsi, G.; Uzel, P.A.; Bourgeois, N.; le François, T.; Rouvillain, J.L.; Bourges, X.; Baroth, S. New injectable bone substitute using reversible thermosensitive hydrogel and BCP granules: In vivo rabbit experiments. Key Eng. Mater. 2009, 396-398, 457-460.

847. Iooss, P.; le Ray, A.M.; Grimandi, G.; Daculsi, G.; Merle, C. A new injectable bone substitute combining poly(e-caprolactone) microparticles with biphasic calcium phosphate granules. Biomaterials 2001, 22, 2785-2794.

848. Bohner, M. Design of ceramic-based cements and putties for bone graft substitution. Eur. Cell Mater. 2010, 20, 1-12.

849. Evis, Z.; Ergun, C.; Doremus, R.H. Hydroxylapatite-zirconia composites: thermal stability of phases and sinterability as related to the $\mathrm{CaO}-\mathrm{ZrO}_{2}$ phase diagram. J. Mater. Sci. 2005, 40, 11271134.

850. Rao, R.R.; Kannan, T.S. Synthesis and sintering of hydroxyapatite-zirconia composites. Mater. Sci. Eng. C 2002, 20, 187-193.

851. Mansur, C.; Pope, M.; Pascucci, M.R.; Shivkumar, S. Zirconia-calcium phosphate composites for bone replacement. Ceram. Int. 1998, 24, 77-79.

852. Kim, H.W.; Kim, H.E.; Salih, V.; Knowles, J.C. Dissolution control and cellular responses of calcium phosphate coatings on zirconia porous scaffold. J. Biomed. Mater. Res. A 2004, 68A, 522-530.

853. Milella, E.; Cosentino, F.; Licciulli, A.; Massaro, C. Preparation and characterisation of titania/hydroxyapatite composite coatings obtained by sol-gel process. Biomaterials 2001, 22, $1425-1431$.

854. Goller, G.; Demirkiran, H.; Oktar, F.N.; Demirkesen, E. Processing and characterization of Bioglass reinforced hydroxyapatite composites. Ceram. Int. 2003, 29, 721-724.

855. Tancred, D.C.; Carr, A.J.; McCormack, B.A. The sintering and mechanical behaviour of hydroxyapatite with bioglass additions. J. Mater. Sci. Mater. Med. 2001, 12, 81-93.

856. Lopes, M.A.; Silva, R.F.; Monteiro, F.J.; Santos, J.D. Microstructural dependence of Young's moduli of $\mathrm{P}_{2} \mathrm{O}_{5}$ glass reinforced hydroxyapatite for biomedical applications. Biomaterials 2000, 21, 749-754.

857. Juang, H.Y.; Hon, M.H. Fabrication and mechanical properties of hydroxyapatite-alumina composites. Mater. Sci. Eng. C 1994, 2, 77-81.

858. Li, J.; Forbreg, S.; Hermansson, L. Evaluation of the mechanical properties of hot isotatically pressed titania and titania-calcium phosphate composites. Biomaterials 1991, 12, 438-440.

859. Noma, T.; Shoji, N.; Wada, S.; Suzuki, T. Preparation of spherical $\mathrm{Al}_{2} \mathrm{O}_{3}$ particle dispersed hydroxyapatite ceramics. J. Ceram. Soc. Jpn. 1993, 101, 923-927.

860. Gautier, S.; Champion, E.; Bernache-Assollant, D. Toughening characterization in alumina platelet-hydroxyapatite matrix composites. J. Mater. Sci. Mater. Med. 1999, 10, 533-540.

861. Fang, Y.; Roy, D.M.; Cheng, J.; Roy. R.; Agrawal, D.K. Microwave sintering of hydroxyapatite-based composites. Ceram. Trans. 1993, 36, 397-407. 
862. Park, K.; Vasilosa, T. Microstructure and mechanical properties of silicon carbide whisker/calcium phosphate composites produced by hot pressing. Mater. Lett. 1997, 32, 229-233.

863. Jin, H.B.; Oktar, F.N.; Dorozhkin, S.; Agathopoulos, S. Sintering behavior and properties of reinforced hydroxyapatite/TCP biphasic bioceramics with ZnO-whiskers. J. Compos. Mater. 2011, 45, 1435-1445.

864. De With, G.; Corbijn, A.T. Metal fibre reinforced hydroxyapatite ceramics. J. Mater. Sci. 1989, 24, 3411-3415.

865. Ruys, A.J.; Simpson, S.A.; Sorrell, C.C. Thixotropic casting of fibre-reinforced ceramic matrix composites. J. Mater. Sci. Lett. 1994, 13, 1323-1325.

866. Miao, X.; Ruys, A.J.; Milthorpe, B.K. Hydroxyapatite-316L fibre composites prepared by vibration assisted slip casting. J. Mater. Sci. 2001, 36, 3323-3332.

867. Kim, H.M.; Chae, W.P.; Chang, K.W.; Chun, S.; Kim, S.; Jeong, Y.; Kang, I.K. Composite nanofiber mats consisting of hydroxyapatite and titania for biomedical applications. J. Biomed. Mater. Res. B Appl. Biomater. 2010, 94, 380-387.

868. Hirakura, S.; Kobayashi, T.; Ono, S.; Oaki, Y.; Imai, H. Fibrous nanocrystals of hydroxyapatite loaded with $\mathrm{TiO}_{2}$ nanoparticles for the capture and photocatalytic decomposition of specific proteins. Colloids Surf. B 2010, 79, 131-135.

869. Wu, J.M.; Yeh, T.S. Sintering of hydroxylapatite-zirconia composite materials. J. Mater. Sci. 1988, 23, 3771-3777.

870. Li, J.; Liao, H.; Hermansson, L. Sintering of partially-stabilized zirconia and partially-stabilized zirconia-hydroxyapatite composites by hot isostatic pressing and pressureless sintering. Biomaterials 1996, 17, 1787-1790.

871. Takagi, M.; Mochida, M.; Uchida, N.; Saito, K.; Uematsu, K. Filter cake forming and hot isostatic pressing for TZP-dispersed hydroxyapatite composite. J. Mater. Sci. Mater. Med. 1992, 3, 199-203.

872. Shen, Z.; Adolfsson, E.; Nygren, M.; Gao, L.; Kawaoka, H.; Niihara, K. Dense hydroxyapatite-zirconia ceramic composites with high strength for biological applications. Adv. Mater. 2001, 13, 214-216.

873. Kumar, B.R.; Prakash, K.H.; Cheang, P.; Khor, K.A. Microstructure and mechanical properties of spark plasma sintered zirconia-hydroxyapatite nano-composite powders. Acta Mater. 2005, 53, 2327-2335.

874. Nagarajan, V.S.; Rao, K.J. Structural, mechanical and biocompatibility studies of hydroxyapatitederived composites toughened by zirconia addition. J. Mater. Chem. 1993, 3, 43-51.

875. Evis, Z.; Doremus, R.H. Hot-pressed hydroxylapatite/monoclinic zirconia composites with improved mechanical properties. J. Mater. Sci. 2007, 42, 2426-2431.

876. Erkmen, Z.E.; Genç, Y.; Oktar, F.N. Microstructural and mechanical properties of hydroxyapatite-zirconia composites. J. Am. Ceram. Soc. 2007, 90, 2885-2892.

877. Rapacz-Kmita, A.; Slosarczyk, A.; Paszkiewicz, Z. Mechanical properties of $\mathrm{HAp}-\mathrm{ZrO}_{2}$ composites. J. Eur. Ceram. Soc. 2006, 26, 1481-1488.

878. Sung, Y.M.; Shin, Y.K.; Ryu, J.J. Preparation of hydroxyapatite/zirconia bioceramic nanocomposites for orthopaedic and dental prosthesis applications. Nanotechnology 2007, 18, 065602:1-065602:6. 
879. Quan, R.; Yang, D.; Wu, X.; Wang, H.; Miao, X.; Li, W. In vitro and in vivo biocompatibility of graded hydroxyapatite-zirconia composite bioceramic. J. Mater. Sci. Mater. Med. 2008, 19, 183187.

880. Khalil, K.A.; Kim, S.W.; Kim, H.Y. Consolidation and mechanical properties of nanostructured hydroxyapatite- $\left(\mathrm{ZrO}_{2}+3 \mathrm{~mol} \% \mathrm{Y}_{2} \mathrm{O}_{3}\right)$ bioceramics by high-frequency induction heat sintering. Mater. Sci. Eng. A 2007, 456, 368-372.

881. Wang, L.L.; Wang, X.F.; Jiang, H.T.; Yu, C.L. Preparation of porous hydroxyapatite-zirconia composite scaffolds by combination of gel-casting and polymer sponge methods. Adv. Mater. Res. 2010, 105-106, 616-619.

882. Li, J.; Fartash, B.; Hermansson, L. Hydroxyapatite-alumina composites and bone-bonding. Biomaterials 1995, 16, 417-422.

883. Adolfsson, E.; Hermansson, L. Phase stability aspects of various apatite-aluminium oxide composites. J. Mater. Sci. 2000, 35, 5719-5723.

884. Jun, Y.K.; Kim, W.H.; Kweon, O.K.; Hong, S.H. The fabrication and biochemical evaluation of alumina reinforced calcium phosphate porous implants. Biomaterials 2003, 24, 3731-3739.

885. Viswanath, B.; Ravishankar, N. Interfacial reactions in hydroxyapatite/alumina nanocomposites. Scripta Mater. 2006, 55, 863-866.

886. Epure, L.M.; Dimitrievska, S.; Merhi, Y.; Yahia, L.H. The effect of varying $\mathrm{Al}_{2} \mathrm{O}_{3}$ percentage in hydroxyapatite $/ \mathrm{Al}_{2} \mathrm{O}_{3}$ composite materials: morphological, chemical and cytotoxic evaluation. $J$. Biomed. Mater. Res. A 2007, 83A, 1009-1023.

887. Evis, Z.; Doremus, R.H. A study of phase stability and mechanical properties of hydroxylapatitenanosize $\alpha$-alumina composites. Mater. Sci. Eng. C 2007, 27, 421-425.

888. Lu, Y.P.; Li, M.S.; Li, S.T.; Wang, Z.G.; Zhu, R.F. Plasma-sprayed hydroxyapatite+titania composite bond coat for hydroxyapatite coating on titanium substrate. Biomaterials 2004, 25, 4393-4403.

889. Lee, S.H.; Kim, H.W.; Lee, E.J.; Li, L.H.; Kim, H.E. Hydroxyapatite-TiO ${ }_{2}$ hybrid coating on Ti implants. J. Biomater. Appl. 2006, 20, 195-208.

890. Boyd, A.R.; Duffy, H.; McCann, R.; Meenan, B.J. Sputter deposition of calcium phosphate/titanium dioxide hybrid thin films. Mater. Sci. Eng. C 2008, 28, 228-236.

891. Fidancevska, E.; Ruseska, G.; Bossert, J.; Linc, Y.M.; Boccaccini, A.R. Fabrication and characterization of porous bioceramic composites based on hydroxyapatite and titania. Mater. Chem. Phys. 2007, 103, 95-100.

892. Pushpakanth, S.; Srinivasan, B.; Sreedhar, B.; Sastry, T.P. An in situ approach to prepare nanorods of titania-hydroxyapatite $\left(\mathrm{TiO}_{2}-\mathrm{HAp}\right)$ nanocomposite by microwave hydrothermal technique. Mater. Chem. Phys. 2008, 107, 492-498.

893. Nath, S.; Tripathi, R.; Basu, B. Understanding phase stability, microstructure development and biocompatibility in calcium phosphate-titania composites, synthesized from hydroxyapatite and titanium powder mix. Mater. Sci. Eng. C 2009, 29, 97-107.

894. Ün, S.; Durucan, C. Preparation of hydroxyapatite-titania hybrid coatings on titanium alloy. $J$. Biomed. Mater. Res. B Appl. Biomater. 2009, 90, 574-583. 
895. Nathanael, A.J.; Mangalaraj, D.; Ponpandian, N. Controlled growth and investigations on the morphology and mechanical properties of hydroxyapatite/titania nanocomposite thin films. Compos. Sci. Technol. 2010, 70, 1645-1651.

896. Ebrahimi-Kahrizsangi, R.; Nasiri-Tabrizi, B.; Chami, A. Synthesis and characterization of fluorapatite-titania $\left(\mathrm{FAp}-\mathrm{TiO}_{2}\right)$ nanocomposite via mechanochemical process. Solid State Sci. 2010, 12, 1645-1651.

897. Sakka, S.; Bouaziz, J.; Ben Ayed, F. Sintering and mechanical properties of the alumina-tricalcium phosphate-titania composites. Mater. Sci. Eng. C 2014, 40, 92-101.

898. Lee, B.T.; Lee, C.W.; Gain, A.K.; Song, H.Y. Microstructures and material properties of fibrous $\mathrm{Ap} / \mathrm{Al}_{2} \mathrm{O}_{3}-\mathrm{ZrO}_{2}$ composites fabricated by multi-pass extrusion process. J. Eur. Ceram. Soc. 2007, 27, 157-163.

899. Oktar, F.N.; Agathopoulos, S.; Ozyegin, L.S.; Gunduz, O.; Demirkol, N.; Bozkurt, Y.; Salman, $\mathrm{S}$. Mechanical properties of bovine hydroxyapatite (BHA) composites doped with $\mathrm{SiO}_{2}, \mathrm{MgO}$, $\mathrm{Al}_{2} \mathrm{O}_{3}$ and $\mathrm{ZrO}_{2}$. J. Mater. Sci. Mater. Med. 2007, 18, 2137-2143.

900. Gunduz, O.; Erkan, E.M.; Daglilar, S.; Salman, S.; Agathopoulos, S.; Okta, F.N. Composites of bovine hydroxyapatite (BHA) and ZnO. J. Mater. Sci. 2008, 43, 2536-2540.

901. Ajeesh, M.; Francis, B.F.; Annie, J.; Varma, P.R.H. Nano iron oxide-hydroxyapatite composite ceramics with enhanced radiopacity. J. Mater. Sci. Mater. Med. 2010, 21, 1427-1434.

902. Yang, Z.P.; Gong, X.Y.; Zhang, C.J. Recyclable $\mathrm{Fe}_{3} \mathrm{O}_{4} /$ hydroxyapatite composite nanoparticles for photocatalytic applications. Chem. Eng. J. 2010, 165, 117-121.

903. Liu, Y.; Zhong, H.; Li, L.; Zhang, C. Temperature dependence of magnetic property and photocatalytic activity of $\mathrm{Fe}_{3} \mathrm{O}_{4} /$ hydroxyapatite nanoparticles. Mater. Res. Bull. 2010, 45, 20362039.

904. Gu, L.; He, X.; Wu, Z. Mesoporous $\mathrm{Fe}_{3} \mathrm{O}_{4}$ /hydroxyapatite composite for targeted drug delivery. Mater. Res. Bull. 2014, 59, 65-68.

905. Dong, L.; Zhu, Z.; Qiu, Y.; Zhao, J. Removal of lead from aqueous solution by hydroxyapatite/manganese dioxide composite. Front. Environ. Sci. Eng. 2015, doi:10.1007/s11783-014-0722-5.

906. Lopes, M.A.; Monterio, F.J.; Santos, J.D. Glass-reinforced hydroxyapatite composites: Fracture toughness and hardness dependence on microstructural characteristics. Biomaterials 1999, 20, 2085-2090.

907. Ragel, C.V.; Vallet-Regi, M.; Rodríguez-Lorenzo, L.M. Preparation and in vitro bioactivity of hydroxyapatite/sol-gel glass biphasic material. Biomaterials 2002, 23, 1865-1872.

908. Li, X.W.; Yasuda, H.Y.; Umakoshi, Y. Bioactive ceramic composites sintered from hydroxyapatite and silica at $1200{ }^{\circ} \mathrm{C}$ : preparation, microstructures and in vitro bone-like layer growth. J. Mater. Sci. Mater. Med. 2006, 17, 573-581.

909. Padilla, S.; Sánchez-Salcedo, S.; Vallet-Regi, M. Bioactive and biocompatible pieces of HA/solgel glass mixtures obtained by the gel-casting method. J. Biomed. Mater. Res. A 2005, 75, 63-72.

910. Padilla, S.; Román, J.; Sánchez-Salcedo, S.; Vallet-Regi, M. Hydroxyapatite/SiO $2-\mathrm{CaO}_{2}-\mathrm{P}_{2} \mathrm{O}_{5}$ glass materials: In vitro bioactivity and biocompatibility. Acta Biomater. 2006, 2, 331-342.

911. Sych, E.E.; Pinchuk, N.D.; Ivanchenko, L.A. Effect of sintering temperature on the properties of biogenic hydroxyapatite-glass composites. Powder Metall. Metal Ceram. 2010, 49, 153-158. 
912. Liu, X.; Ei-Ghannam, A. Effect of processing parameters on the microstructure and mechanical behavior of silica-calcium phosphate nanocomposite. J. Mater. Sci. Mater. Med. 2010, 21, 20872094.

913. Bellucci, D.; Sola, A.; Cacciotti, I.; Bartoli, C.; Gazzarri, M.; Bianco, A.; Chiellini, F.; Cannillo, $\mathrm{V}$. Mg and/or Sr-doped tricalcium phosphate/bioactive glass composites: Synthesis, microstructure and biological responsiveness. Mater. Sci. Eng. C 2014, 42, 312-324.

914. Bellucci, D.; Sola, A.; Lusvarghi, L.; Cannillo, V. Hydroxyapatite-tricalcium phosphate-bioactive glass ternary composites. Ceram. Int. 2014, 40, 3805-3808.

915. Yazdanpanah, Z.; Bahrololoom, M.E.; Hashemi, B. Evaluating morphology and mechanical properties of glass-reinforced natural hydroxyapatite composites. J. Mech. Behav. Biomed. Mater. 2015, 41, 36-42.

916. Kokubo, T.; Shigematsu, M.; Nagashima, Y.; Tashiro, M.; Nakamura, T.; Yamamuro, T.; Higashi, S. Apatite- and wollastonite-containing glass ceramics for prosthetic applications. Bull. Inst. Chem. Res. Kyoto Univ. 1982, 60, 260-268.

917. Kokubo, T.; Ito, S.; Shigematsu, M.; Sakka, S.; Yamamuro, T. Fatigue and life-time of bioactive glass-ceramic A-W containing apatite and wollastonite. J. Mater. Sci. 1987, 22, 4067-4070.

918. Nishio, K.; Neo, M.; Akiyama, H.; Okada, Y.; Kokubo, T.; Nakamura, T. Effects of apatite and wollastonite containing glass-ceramic powder and two types of alumina powder in composites on osteoblastic differentiation of bone marrow cells. J. Biomed. Mater. Res. 2001, 55, 164-176.

919. Encinas-Romero, M.A.; Aguayo-Salinas, S.; Valenzuela-García, J.L.; Payán, S.R.; CastillónBarraza, F.F. Mechanical and bioactive behavior of hydroxyapatite-wollastonite sintered composites. Int. J. Appl. Ceram. Technol. 2010, 7, 164-177.

920. Ha, N.R.; Yang, Z.X.; Hwang, K.H.; Kim, T.S.; Lee, J.K. Improvement of the stability of hydroxyapatite through glass ceramic reinforcement. J. Nanosci. Nanotechnol. 2010, 10, 3459-3462.

921. Nath, S.; Biswas, K.; Wang, K.; Bordia, R.K.; Basu, B. Sintering, phase stability, and properties of calcium phosphate-mullite composites. J. Am. Ceram. Soc. 2010, 93, 1639-1649.

922. Nath, S.; Ummethala, R.; Basu, B. Fretting wear behavior of calcium phosphate-mullite composites in dry and albumin-containing simulated body fluid conditions. J. Mater. Sci. Mater. Med. 2010, 21, 1151-1161.

923. Nath, S.; Dubey, A.K.; Basu, B. Mechanical properties of novel calcium phosphate-mullite biocomposites. J. Biomater. Appl. 2012, 27, 67-78.

924. Nath, S.; Kalmodia, S.; Basu, B. In vitro biocompatibility of novel biphasic calcium phosphatemullite composites. J. Biomater. Appl. 2013, 27, 497-509.

925. Chaki, T.K.; Wang, P.E. Densification and strengthening of silver-reinforced hydroxyapatite-matrix composite prepared by sintering. J. Mater. Sci. Mater. Med. 1994, 5, 533-542.

926. Zhang, X.; Gubbels, G.H.M.; Terpstra, R.A.; Metselaar, R. Toughening of calcium hydroxyapatite with silver particles. J. Mater. Sci. 1997, 32, 235-243.

927. Chu, C.; Lin, P.; Dong, Y.; Xue, X.; Zhu, J.; Yin, Z. Fabrication and characterization of hydroxyapatite reinforced with $20 \mathrm{vol} \%$ Ti particles for use as hard tissue replacement. J. Mater. Sci. Mater. Med. 2002, 13, 985-992. 
928. Ning, C.Q.; Zhou, Y. In vitro bioactivity of a biocomposite fabricated from HA and Ti powders by powder metallurgy method. Biomaterials 2002, 23, 2909-2915.

929. Chu, C.; Xue, X.; Zhu, J.; Yin, Z. Mechanical and biological properties of hydroxyapatite reinforced with 40 vol. \% titanium particles for use as hard tissue replacement. J. Mater. Sci. Mater. Med. 2004, 15, 665-670.

930. Karanjai, M.; Kumarb, M.B.V.; Sundaresan, R.; Basu, B.; Mohan, R.T.R.; Kashyap, B.P. Fretting wear study on Ti-Ca-P biocomposite in dry and simulated body fluid. Mater. Sci. Eng. A 2008, 475, 299-307.

931. Chu, C.; Xue, X.; Zhu, J.; Yin, Z. Fabrication and characterization of titanium-matrix composite with 20 vol. \% hydroxyapatite for use as heavy load-bearing hard tissue replacement. J. Mater. Sci. Mater. Med. 2006, 17, 245-251.

932. Miranda, M.; Fernández, A.; Díaz, M.; Esteban-Tejeda, L.; López-Esteban, S.; Malpartida, F.; Torrecillas, R.; Moya, J.S. Silver-hydroxyapatite nanocomposites as bactericidal and fungicidal materials. Int. J. Mater. Res. 2010, 101, 122-127.

933. Younesi, M.; Bahrololoom, M.E. Optimizations of wear resistance and toughness of hydroxyapatite nickel free stainless steel new bio-composites for using in total joint replacement. Mater. Des. 2010, 31, 234-243.

934. Murakoshi, Y.; Kikuchi, K.; Katoh, M.; Matsuzaki, K. Fabrication and property of degradable magnesium-calcium alloy composites with hydroxyapatite. IFMBE Proc. 2010, 31, 1226-1229.

935. Razavi, M.; Fathi, M.H.; Meratian, M. Fabrication and characterization of magnesium-fluorapatite nanocomposite for biomedical applications. Mater. Character. 2010, 61, 1363-1370.

936. Kumar, A.; Biswas, K.; Basu, B. On the toughness enhancement in hydroxyapatite-based composites. Acta Mater. 2013, 61, 5198-5215.

937. Huang, Y.; Liu, D.B.; Xia, M.; Anguilano, L. Characterization of an Mg-2Zn-1Ca 1ß-TCP composite fabricated by high shear solidification and ECAE. Mater. Sci. Forum 2013, 765, 813817.

938. Sun, J.; Chen, M.; Cao, G.; Bi, Y.; Liu, D.; Wei, J. The effect of nano-hydroxyapatite on the microstructure and properties of $\mathrm{Mg}-3 \mathrm{Zn}-0.5 \mathrm{Zr}$ alloy. J. Compos. Mater. 2014, 48, 825-834.

939. Chang, Q.; Ru, H.Q.; Chen, D.L.; Zhang, C.P.; Yang, J.L.; Hu, S.L. Interfacial reactions in TiFe particles reinforced hydroxyapatite matrix composites. Mater. Lett. 2014, 128, 245-247.

940. Sunil, B.R.; Kumar, T.S.S.; Chakkingal, U.; Nandakumar, V.; Doble, M. Friction stir processing of magnesium-nanohydroxyapatite composites with controlled in vitro degradation behavior. Mater. Sci. Eng. C 2014, 39, 315-324.

941. Buyong, S.A.; Jamaludin, S.B.; Malek, R.A. Effect of beta tricalcium phosphate ( $\beta$-TCP) on properties of Mg-Zn composites. Key Eng. Mater. 2014, 594-595, 203-206.

942. Arifin, A.; Sulong, A.B.; Muhamad, N.; Syarif, J.; Ramli, M.I. Material processing of hydroxyapatite and titanium alloy (HA/Ti) composite as implant materials using powder metallurgy: a review. Mater. Des. 2014, 55, 165-175.

943. Wakily, H.; Dabbagh, A.; Abdullah, H.; Abdul Halim, N.F.; Abu Kasim, N.H. Improved thermal and mechanical properties in hydroxyapatite - titanium composites by incorporating silica-coated titanium. Mater. Lett. 2015, 143, 322-325. 
944. Damien, C.J.; Parsons, J.R.; Benedict, J.J.; Weisman, D.S. Investigation of a hydroxyapatite and calcium sulfate composite supplemented with an osteoinductive factor. J. Biomed. Mater. Res. 1990, 24, 639-654.

945. Rauschmann, M.A.; Wichelhaus, T.A.; Stirnal, V.; Dingeldein, E.; Zichner, L.; Schnettler, R.; Alt, V. Nanocrystalline hydroxyapatite and calcium sulphate as biodegradable composite carrier material for local delivery of antibiotics in bone infections. Biomaterials 2005, 26, 2677-2684.

946. Urban, R.M.; Turner, T.M.; Hall, D.J.; Inoue, N.; Gitelis, S. Increased bone formation using calcium sulfate-calcium phosphate composite graft. Clin. Orthop. Relat. Res. 2007, 459, 110-117.

947. Rauschmann, M.; Vogl, T.; Verheyden, A.; Pflugmacher, R.; Werba, T.; Schmidt, S.; Hierholzer, J. Bioceramic vertebral augmentation with a calcium sulphate/hydroxyapatite composite (Cerament ${ }^{\mathrm{TM}}$ SpineSupport) in vertebral compression fractures due to osteoporosis. Eur. Spine J. 2010, 19, 887-892.

948. Nilsson, M.; Zheng, M.H.; Tägil, M. The composite of hydroxyapatite and calcium sulphate: A review of preclinical evaluation and clinical applications. Expert Rev. Med. Dev. 2013, 10, 675684.

949. Du, M.K.; Kuang, Z.D.; Ji, H.R.; Mao, K.Y. Enhanced degradation and osteogenesis of $\beta$-tricalcium phosphate/calcium sulfate composite bioceramics: the effects of phase ratio. $J$. Biomater. Tissue Eng. 2014, 4, 389-398.

950. Kumar, G.S.; Girija, E.K.; Thamizhavel, A.; Yokogawa, Y.; Kalkura, S.N. Synthesis and characterization of bioactive hydroxyapatite-calcite nanocomposite for biomedical applications. J. Colloid Interf. Sci. 2010, 349, 56-62.

951. Smirnov, V.V.; Goldberg, M.A.; Shvorneva, L.I.; Fadeeva, I.V.; Shibaeva, T.V.; Barinov, S.M. Synthesis of composite biomaterials in the hydroxyapatite-calcite system. Doklady Chem. 2010, 432, 151-154.

952. Gittings, J.P.; Bowena, C.R.; Turner, I.G.; Baxter, F.; Chaudhuri, J. Characterisation of ferroelectric-calcium phosphate composites and ceramics. J. Eur. Ceram. Soc. 2007, 27, 41874190.

953. Watanabe, Y.; Ikoma, T.; Suetsugu, Y.; Yamada, H.; Tamura, K.; Komatsu, Y.; Tanaka, J.; Moriyoshi, Y. The densification of zeolite/apatite composites using a pulse electric current sintering method: a long-term assurance material for the disposal of radioactive waste. $J$. Eur. Ceram. Soc. 2006, 26, 481-486.

954. Lahiri, D.; Singh, V.; Benaduce, A.P.; Seal, S.; Kos, L.; Agarwal, A. Boron nitride nanotube reinforced hydroxyapatite composite: mechanical and tribological performance and in vitro biocompatibility to osteoblasts. J. Mech. Behav. Biomed. Mater. 2011, 4, 44-56.

955. Nathanael, A.J.; Yuvakkumar, R.; Hong, S.I.; Oh, T.H. Novel zirconium nitride and hydroxyapatite nanocomposite coating: detailed analysis and functional properties. ACS Appl. Mater. Interf. 2014, 6, 9850-9857.

956. Liu, Y.; Huang, J.; Li, H. Synthesis of hydroxyapatite-reduced graphite oxide nanocomposites for biomedical applications: oriented nucleation and epitaxial growth of hydroxyapatite. J. Mater. Chem. B 2013, 1, 1826-1834.

957. Agathopoulos, S.; Tulyaganov, D.U.; Marques, P.A.A.P.; Ferro, M.C.; Fernandes, M.H.V.; Correia, R.N. The fluorapatite-anorthite system in biomedicine. Biomaterials 2003, 24, 1317-1331. 
958. Khor, K.A.; Gu, Y.W.; Pan, D.; Cheang, P. Microstructure and mechanical properties of plasma sprayed HA/YSZ/Ti-6Al-4V composite coatings. Biomaterials 2004, 25, 4009-4017.

959. Gu, Y.W.; Khor, K.A.; Pan, D.; Cheang, P. Activity of plasma sprayed yttria stabilized zirconia reinforced hydroxyapatite/Ti-6Al-4V composite coatings in simulated body fluid. Biomaterials 2004, 25, 3177-3185.

960. Zhang, Y.; Chen, L.; Zeng, J.; Zhou, K.; Zhang, D. Aligned porous barium titanate/hydroxyapatite composites with high piezoelectric coefficients for bone tissue engineering. Mater. Sci. Eng. C 2014, 39, 143-149.

961. Kalmodia, S.; Goenka, S.; Laha, T.; Lahiri, D.; Basu, B.; Balani, K. Microstructure, mechanical properties, and in vitro biocompatibility of spark plasma sintered hydroxyapatite-aluminum oxide-carbon nanotube composite. Mater. Sci. Eng. C 2010, 30, 1162-1169.

962. Best, S.M.; Porter, A.E.; Thian, E.S.; Huang, J. Bioceramics: past, present and for the future. $J$. Eur. Ceram. Soc. 2008, 28, 1319-1327.

963. De Aza, P.N.; Guitián, F.; de Aza, S. Bioeutectic: A new ceramic material for human bone replacement. Biomaterials 1997, 18, 1285-1291.

964. Huang, X.; Jiang, D.; Tan, S. Apatite formation on the surface of wollastonite/tricalcium phosphate composite immersed in simulated body fluid. J. Biomed. Mater. Res. B Appl. Biomater. 2004, 69, 70-72.

965. Zhang, F.; Chang, J.; Lin, K.; Lu, J. Preparation, mechanical properties and in vitro degradability of wollastonite/tricalcium phosphate macroporous scaffolds from nanocomposite powders. J. Mater. Sci. Mater. Med. 2008, 19, 167-173.

966. Juhasz, J.A.; Best, S.M.; Kawashita, M.; Miyata, N.; Kokubo, T.; Nakamura, T.; Bonfield, W. Bonding strength of the apatite layer formed on glass-ceramic apatite-wollastonite-polyethylene composites. J. Biomed. Mater. Res. A 2003, 67A, 952-959.

967. Juhasz, J.A.; Best, S.M.; Bonfield, W.; Kawashita, M.; Miyata, N.; Kokubo, T.; Nakamura, T. Apatite-forming ability of glass-ceramic apatite-wollastonite - polyethylene composites: Effect of filler content. J. Mater. Sci. Mater. Med. 2003, 14, 489-495.

968. Juhasz, J.A.; Best, S.M.; Brooks, R.; Kawashita, M.; Miyata, N.; Kokubo, T.; Nakamura, T.; Bonfield, W. Mechanical properties of glass-ceramic A-W-polyethylene composites: Effect of filler content and particle size. Biomaterials 2004, 25, 949-955.

969. Rea, S.M.; Brooks, R.A.; Best, S.M.; Kokubo, T.; Bonfield, W. Proliferation and differentiation of osteoblast-like cells on apatite-wollastonite/polyethylene composites. Biomaterials 2004, 25, 4503-4512.

970. Zhao, S.; Zhou, Z.; Wu, J.; Wang, S.; Guo, X.; Zhang, Q. Preparation and characterization of a novel hydroxyapatite-wollastonite/silk fibroin composite. J. Compos. Mater. 2012, 46, 15711581 .

971. Greish, Y.E.; Brown, P.W. Characterization of wollastonite-reinforced HAp-Ca polycarboxylate composites. J. Biomed. Mater. Res. 2001, 55, 618-628.

972. Greish, Y.E.; Brown, P.W. Characterization of bioactive glass-reinforced HAP-polymer composites. J. Biomed. Mater. Res. 2000, 52, 687-694. 
973. Radev, L.; Hristov, V.; Samuneva, B.; Ivanova, D. Organic/inorganic bioactive materials. Part II: in vitro bioactivity of collagen-calcium phosphate silicate/wollastonite hybrids. Central Eur. J. Chem. 2009, 7, 711-720.

974. Radev, L.; Hristov, V.; Fernandes, M.H.V.; Salvado, I.M.M. Organic/inorganic bioactive materials. Part IV: In vitro assessment of bioactivity of gelatin-calcium phosphate silicate/wollastonite hybrids. Central Eur. J. Chem. 2010, 8, 278-284.

975. Kangasniemi, I.; de Groot, K.; Wolke, J.; Andersson, O.; Luklinska, Z.; Becht, J.G.M.; Lakkisto, M.; Yli-Urpo, A. The stability of hydroxyapatite in an optimized bioactive glass matrix at sintering temperatures. J. Mater. Sci. Mater. Med. 1991, 2, 133-137.

976. Kangasniemi, I.M.O.; de Groot, K.; Becht, J.G.M.; Yli-Urpo, A. Preparation of dense hydroxylapatite or rhenanite containing bioactive glass composites. J. Biomed. Mater. Res. 1992, 26, 663-674.

977. Kangasniemi, I.M.O.; Vedel, E.; de Blick-Hogerworst, J.; Yli-Urpo. A.; de Groot, K. Dissolution and scanning electron microscopic studies of $\mathrm{Ca}, \mathrm{P}$ particle-containing bioactive glasses. J. Biomed. Mater. Res. 1993, 27, 1225-1233.

978. Maruno, S.; Ban, S.; Wang, Y.F.; Iwata, H.; Itoh, H. Properties of functionally gradient composite consisting of hydroxyapatite containing glass coated titanium and characters for bioactive implant. J. Ceram. Soc. Jpn. 1992, 100, 362-367.

979. White, A.A.; Best, S.M.; Kinloch, I.A. Hydroxyapatite-carbon nanotube composites for biomedical applications: a review. Int. J. Appl. Ceram. Technol. 2007, 4, 1-13.

980. Zhao, L.P.; Gao, L. Novel in situ synthesis of MWNT-hydroxyapatite composites. Carbon 2004, 42, 423-460.

981. Wei, Q.; Yang, X.P.; Chen, G.Q.; Tang, J.T.; Deng, X.L. The ultrasonic assisted synthesis of nano-hydroxyapatite and MWNT/hydroxyapatite composites. New Carbon Mater. 2005, 20, 164-170.

982. Bai, Y.; Neupane, M.P.; Park, I.S.; Lee, M.H.; Bae, T.S.; Watari, F.; Uo, M. Electrophoretic deposition of carbon nanotubes-hydroxyapatite nanocomposites on titanium substrate. Mater. Sci. Eng. C 2010, 30, 1043-1049.

983. Shin, U.S.; Yoon, I.K.; Lee, G.S.; Jang, W.C.; Knowles, J.C.; Kim, H.W. Carbon nanotubes in nanocomposites and hybrids with hydroxyapatite for bone replacements. J. Tissue Eng. 2011, 2011, 674287:1-674287:10.

984. Lahiri, D.; Ghosh, S.; Agarwal, A. Carbon nanotube reinforced hydroxyapatite composite for orthopedic application: a review. Mater. Sci. Eng. C 2012, 32, 1727-1758.

985. Kobayashi, S.; Kawai, W. Development of carbon nanofiber reinforced hydroxyapatite with enhanced mechanical properties. Composites A 2007, 38, 114-123.

986. Sopyan, I.; Nurfaezah, S.; Ammar, M. Development of triphasic calcium phosphate-carbon nanotubes (HA/TCP-CNT) composite: A preliminary study. Key Eng. Mater. 2013, 531-532, 258-261.

987. Balani, K.; Anderson, R.; Laha, T.; Andara, M.; Tercero, J.; Crumpler, E.; Agarwal, A. Plasma-sprayed carbon nanotube reinforced hydroxyapatite coatings and their interaction with human osteoblasts in vitro. Biomaterials 2007, 28, 618-624. 
988. Chen, Y.; Gan, C.H.; Zhang, T.H.; Yu, G.; Bai, P.; Kaplan, A. Laser-surface-alloyed carbon nanotubes reinforced hydroxyapatite composite coatings. Appl. Phys. Lett. 2005, 86, 251905:1-251905:3.

989. Chen, Y.; Zhang, T.H.; Gan, C.H.; Yu, G. Wear studies of hydroxyapatite composite coating reinforced by carbon nanotubes. Carbon 2007, 45, 998-1004.

990. Chen, Y.; Zhang, Y.Q.; Zhang, T.H.; Gan, C.H.; Zheng, C.Y.; Yu, G. Carbon nanotube reinforced hydroxyapatite composite coatings produced through laser surface alloying. Carbon 2006, 44, $37-45$.

991. Xu, J.L.; Khor, K.A.; Sui, J.J.; Chen, W.N. Preparation and characterization of a novel hydroxyapatite/carbon nanotubes composite and its interaction with osteoblast-like cells. Mater. Sci. Eng. C 2009, 29, 44-49.

992. Mukherjee, S.; Kundu, B.; Sen, S.; Chanda, A. Improved properties of hydroxyapatite-carbon nanotube biocomposite: mechanical, in vitro bioactivity and biological studies. Ceram. Int. 2014, $40,5635-5643$.

993. Hooshmand, T.; Abrishamchian, A.; Najafi, F.; Mohammadi, M.; Najafi, H.; Tahriri, M. Development of sol-gel-derived multi-wall carbon nanotube/hydroxyapatite nanocomposite powders for bone substitution. J. Compos. Mater. 2014, 48, 483-489.

994. Lee, H.H.; Shin, U.S.; Won, J.E.; Kim, H.W. Preparation of hydroxyapatite-carbon nanotube composite nanopowders. Mater. Lett. 2010, 65, 208-211.

995. White, A.A.; Kinloch, I.A.; Windle, A.H.; Best, S.M. Optimization of the sintering atmosphere for high-density hydroxyapatite-carbon nanotube composites. J. Royal Soc. Interf. 2010, 7, S529-S539.

996. Ding, Y.; Liu, J.; Jin, X.; Lu, H.; Shen, G.; Yu, R. Poly-L-lysine/hydroxyapatite/carbon nanotube hybrid nanocomposite applied for piezoelectric immunoassay of carbohydrate antigen 19-9. Analyst 2008, 133, 184-190.

997. Zhao, H.Y.; Xu, X.X.; Zhang, J.X.; Zheng, W.; Zheng, Y.F. Carbon nanotube-hydroxyapatitehemoglobin nanocomposites with high bioelectrocatalytic activity. Bioelectrochemistry 2010, 78, 124-129.

998. Slosarcyk, A.; Klisch, M.; Blazewicz, M.; Piekarczyk, J.; Stobierski, L.; Rapacz-Kmita, A. Hot pressed hydroxyapatite-carbon fibre composites. J. Eur. Ceram. Soc. 2000, 20, 1397-1402.

999. Dorner-Reisel, A.; Berroth, K.; Neubauer, R.; Nestler, K.; Marx, G.; Scislo, M.; Müller, E.; Slosarcyk, A. Unreinforced and carbon fibre reinforced hydroxyapatite: resistance against microabrasion. J. Eur. Ceram. Soc. 2004, 24, 2131-2139.

1000. Fu, T.; Zhao, J.L.; Wei, J.H.; Han, Y.; Xu, K.W. Preparation of carbon fiber fabric reinforced hydroxyapatite/epoxy composite by RTM processing. J. Mater. Sci. 2004, 39, 1411-1413.

1001. Pecheva, E.; Pramatarova, L.; Hikov, T.; Fingarova, D.; Tanaka, Y.; Sakamoto, H.; Doi, H.; Tsutsumi, Y.; Hanawa, T. Apatite-nanodiamond composite as a functional coating of stainless steel. Surf. Interf. Anal. 2010, 42, 475-480.

1002. Liu, Y.; Dang, Z.; Wang, Y.; Huang, J.; Li, H. Hydroxyapatite/graphene-nanosheet composite coatings deposited by vacuum cold spraying for biomedical applications: Inherited nanostructures and enhanced properties. Carbon 2014, 67, 250-259. 
1003. Baradaran, S.; Moghaddam, E.; Basirun, W.J.; Mehrali, M.; Sookhakian, M.; Hamdi, M.; Moghaddam, M.R.N.; Alias, Y. Mechanical properties and biomedical applications of a nanotube hydroxyapatite-reduced graphene oxide composite. Carbon 2014, 69, 32-45.

1004. Zhang, L.; Zhang, X.G.; Chen, Y.; Su, J.N.; Liu, W.W.; Zhang, T.H.; Qi, F.; Wang, Y.G. Interfacial stress transfer in a graphene nanosheet toughened hydroxyapatite composite. Appl. Phys. Lett. 2014, 105, 161908.

1005. Azhari, A.; Toyserkani, E.; Villain, C. Additive manufacturing of graphene-hydroxyapatite nanocomposite structures. Int. J. Appl. Ceram. Technol. 2015, 12, 8-17.

1006. Janković, A.; Eraković, S.; Mitrić, M.; Matić, I.Z.; Juranić, Z.D.; Tsui, G.C.P.; Tang, C.Y.; Mišković-Stanković, V.; Rhee, K.Y.; Park, S.J. Bioactive hydroxyapatite/graphene composite coating and its corrosion stability in simulated body fluid. J. Alloy Compd. 2015, 624, 148-157.

1007. Klebert, S.; Balazsi, C.; Balazsi, K.; Bodis, E.; Fazekas, P.; Keszler, A.M.; Szepvolgyi, J.; Karoly, Z. Spark plasma sintering of graphene reinforced hydroxyapatite composites. Ceram. Int. 2015, 41, 3647-3652.

1008. Djordjevic, A.; Ignjatovic, N.; Seke, M.; Jovic, D.; Uskokovic, D.; Rakocevic, Z. Synthesis and characterization of hydroxyapatite/fullerenol nanocomposites. J. Nanosci. Nanotechnol. 2015, $15,1538-1542$.

1009. Yoshimura, M. Phase stability of zirconia. Am. Ceram. Soc. Bull. 1988, 67, 1950-1955.

1010. Thompson, I.; Rawlings, R.D. Mechanical behaviour of zirconia and zirconia-toughened alumina in a simulated body environment. Biomaterials 1990, 11, 505-508.

1011. Egorov, A.; Smirnov, V.; Shvorneva, L.; Kutsev, S.; Barinov, S. High-temperature hydroxyapatite-titanium interaction. Inorg. Mater. 2010, 46, 168-171.

1012. You, C.; Bi, Y.; Chen, M.F.; Sun, Y.; Liu, D.B. Effect of the addition of nano- $\beta$-TCP on the microstructure of Mg-Zn-Zr alloy. Adv. Mater. Res. 2012, 535-537, 259-263.

1013. Monma, H. Tricalcium phosphate ceramics complexed with hydroxyapatite. J. Ceram. Soc. Jpn. 1987, 96, 60-64.

1014. Farzadi, A.; Solati-Hashjin, M.; Bakhshi, F.; Aminian, A. Synthesis and characterization of hydroxyapatite/ $\beta$-tricalcium phosphate nanocomposites using microwave irradiation. Ceram. Int. 2011, 37, 65-71.

1015. Wu, C.C.; Huang, S.T.; Tseng, T.W.; Rao, Q.L.; Lin, H.C. FT-IR and XRD investigations on sintered fluoridated hydroxyapatite composites. J. Mol. Struct. 2010, 979, 72-76.

1016. Suchanek, W.; Yashima, M.; Kakihana, M.; Yoshimura, M. Processing and mechanical properties of hydroxyapatite reinforced with hydroxyapatite whiskers. Biomaterials 1996, 17, 1715-1723.

1017. Suchanek, W.; Yashima, M.; Kakihana, M.; Yoshimura, M. Hydroxyapatite/hydroxyapatite-whisker composites without sintering additives: mechanical properties and microstructural evolution. $J$. Am. Ceram. Soc. 1997, 80, 2805-2813.

1018. Kaito, T.; Mukai, Y.; Nishikawa, M.; Ando, W.; Yoshikawa, H.; Myoui, A. Dual hydroxyapatite composite with porous and solid parts: Experimental study using canine lumbar interbody fusion model. J. Biomed. Mater. Res. B Appl. Biomater. 2006, 78B, 378-384.

1019. Ramay, H.R.; Zhang, M. Biphasic calcium phosphate nanocomposite porous scaffolds for loadbearing bone tissue engineering. Biomaterials 2004, 21, 5171-5180. 
1020. Kobayashi, S.; Murakoshi, T. Characterization of mechanical properties and bioactivity of hydroxyapatite/ $\beta$-tricalcium phosphate composites. Adv. Compos. Mater. 2014, 23, 163-177.

1021. Matsumoto, K.; Tsuru, K.; Kawachi, G.; Maruta, M.; Matsuya, S.; Takahashi, I.; Ishikawa, K. Reinforcement of carbonate apatite bone substitutes with carbonate apatite by $\mathrm{Ca}$ salt introduction. J. Ceram. Soc. Jpn. 2010, 118, 521-524.

1022. Tampieri, A.; Celotti, G.; Sprio, S.; Delcogliano, A.; Franzese, S. Porosity-graded hydroxyapatite ceramics to replace natural bone. Biomaterials 2001, 22, 1365-1370.

1023. Werner, J.; Linner-Krcmar, B.; Friess, W.; Greil, P. Mechanical properties and in vitro cell compatibility of hydroxyapatite ceramics with graded pore structure. Biomaterials 2002, 23, 4285-4294.

1024. Hsu, Y.H.; Turner, I.G.; Miles, A.W. Fabrication of porous bioceramics with porosity gradients similar to the bimodal structure of cortical and cancellous bone. J. Mater. Sci. Mater. Med. 2007, $18,2251-2256$.

1025. Macchetta, A.; Turner, I.G.; Bowen, C.R. Fabrication of HA/TCP scaffolds with a graded and porous structure using a camphene-based freeze-casting method. Acta Biomater. 2009, 5, 13191327.

1026. Watari, F.; Yokoyama, A.; Saso, F.; Uo, M.; Kawasaki, T. Fabrication and properties of functionally graded dental implant. Composites B 1997, 28B, 5-11.

1027. Watari, F.; Yokoyama, A.; Omori, M.; Hirai, T.; Kondo, H.; Uo, M.; Kawasaki, T. Biocompatibility of materials and development to functionally graded implant for bio-medical application. Compos. Sci. Technol. 2004, 64, 893-908.

1028. Chu, C.; Zhu, J.; Yin, Z.; Wang, S. Hydroxyapatite-Ti functionally graded biomaterial fabricated by powder metallurgy. Mater. Sci. Eng. A 1999, 271, 95-100.

1029. Chu, C.; Zhu, J.; Yin, Z.; Lin, P. Structure optimization and properties of hydroxyapatite-Ti symmetrical functionally graded biomaterial. Mater. Sci. Eng. A 2001, 316, 205-210.

1030. Chu, C.; Zhu, J.; Yin, Z.; Lin, P. Optimal design and fabrication of hydroxyapatite-Ti asymmetrical functionally graded biomaterial. Mater. Sci. Eng. A 2003, 348, 244-250.

1031. Bai, X.; More, K.; Rouleau, C.M.; Rabiei, A. Functionally graded hydroxyapatite coatings doped with antibacterial components. Acta Biomater. 2010, 6, 2264-2273.

1032. Inagaki, M.; Yokogawa, Y.; Kameyama, T. Effects of plasma gas composition on bond strength of hydroxyapatite/titanium composite coatings prepared by rf-plasma spraying. J. Eur. Ceram. Soc. 2006, 26, 495-499.

1033. Pei, X.; Wang, J.; Wan, Q.; Kang, L.; Xiao, M.; Bao, H. Functionally graded carbon nanotubes/hydroxyapatite composite coating by laser cladding. Surf. Coat. Technol. 2011, 205, 4380-4387.

1034. Boanini, E.; Torricelli, P.; Sima, F.; Axente, E.; Fini, M.; Mihailescu, I.N.; Bigi, A. Strontium and zoledronate hydroxyapatites graded composite coatings for bone prostheses. J. Colloid Interf. Sci. 2015, 448, 1-7.

1035. Liu, C.; Han, Z.; Czernuszka, J.T. Gradient collagen/nanohydroxyapatite composite scaffold: development and characterization. Acta Biomater. 2009, 5, 661-669. 
1036. Jamuna-Thevi, K.; Saarani, N.N.; Kadir, M.R.A.; Hermawan, H. Triple-layered PLGA/nanoapatite/lauric acid graded composite membrane for periodontal guided bone regeneration. Mater. Sci. Eng. C 2014, 43, 253-263.

1037. Erisken, C.; Kalyon, D.M.; Wang, H. Functionally graded electrospun polycaprolactone and $\beta$ tricalcium phosphate nanocomposites for tissue engineering applications. Biomaterials 2008, 29, 4065-4073.

1038. Nindhia, T.G.T.; Koyoshi, Y.; Kaneko, A.; Sawada, H.; Ohta, M.; Hirai, S.; Uo, M. Hydroxyapatite-silk functionally graded material by pulse electric current sintering. Trends Biomater. Artif. Organs 2008, 22, 25-29.

1039. Ban, S.; Hasegawa, J.; Maruno, S. Fabrication and properties of functionally gradient bioactive composites comprising hydroxyapatite containing glass coated titanium. Mater. Sci. Forum 1999, 308-311, 350-355.

1040. Stojanovic, D.; Jokic, B.; Veljovic, D.; Petrovic, R.; Uskokovic, P.S.; Janackovic, D. Bioactive glass-apatite composite coating for titanium implant synthesized by electrophoretic deposition. J. Eur. Ceram. Soc. 2007, 27, 1595-1599.

1041. Wong, L.H.; Tio, B.; Miao, X. Functionally graded tricalcium phosphate/fluoroapatite composites. Mater. Sci. Eng. C 2002, 20, 111-115.

1042. Afzal, M.A.F.; Kesarwani, P.; Reddy, K.M.; Kalmodia, S.; Basu, B.; Balani, K. Functionally graded hydroxyapatite-alumina-zirconia biocomposite: synergy of toughness and biocompatibility Mater. Sci. Eng. C 2012, 32, 1164-1173.

1043. Okazaki, M.; Miake, Y.; Tohda, H.; Yanagisawa, T.; Matsumoto, T.; Takahashi, J. Functionally graded fluoridated apatites. Biomaterials 1999, 20, 1421-1426.

1044. Okazaki, M.; Takahashi, J. Synthesis of functionally graded $\mathrm{CO}_{3}$ apatite as surface biodegradable crystals. Biomaterials 1999, 20, 1073-1078.

1045. Peltola, T.; Patsi, M.; Rahiala, H.; Kangasniemi, I.; Yli-Urpo, A. Calcium phosphate induction by sol-gel-derived titania coatings on titanium substrates in vitro. J. Biomed. Mater. Res. 1998, 41, 504-510.

1046. Heilmann, F.; Standard, O.C.; Müller, F.A.; Hoffman, M. Development of graded hydroxyapatite $/ \mathrm{CaCO}_{3}$ composite structures for bone ingrowth. J. Mater. Sci. Mater. Med. 2007, $18,1817-1824$.

1047. Katti, K.S. Biomaterials in total joint replacement. Colloids Surf. B 2004, 39, 133-142.

1048. Cavalcanti, A.; Shirinzadeh, B.; Zhang, M.; Kretly, L.C. Nanorobot hardware architecture for medical defense. Sensors 2008, 8, 2932-2958.

1049. Zhang, H.; Xu, J.J.; Chen, H.Y. Electrochemically deposited 2D nanowalls of calcium phosphatePDDA on a glassy carbon electrode and their applications in biosensing. J. Phys. Chem. C 2007, 111, 16564-16570.

1050. Ding, Y.; Liu, J.; Wang, H.; Shen, G.; Yu, R. A piezoelectric immunosensor for the detection of $\alpha$-fetoprotein using an interface of gold/hydroxyapatite hybrid nanomaterial. Biomaterials 2007, $28,2147-2154$.

1051. Ding, Y.; Liu, J.; Jin, X.; Shen, G.; Yu, R. A novel piezoelectric immunosensor for CA125 using a hydroxyapatite/chitosan nanocomposite-based biomolecular immobilization method. Aust. J. Chem. 2008, 61, 500-505. 
1052. López, M.S.P.; Tamimi, F.; López-Cabarcos, E.; López-Ruiz, B. Highly sensitive amperometric biosensor based on a biocompatible calcium phosphate cement. Biosens. Bioelectron. 2009, 24, 2574-2579.

1053. Wang, B.; Zhang, J.J.; Pan, Z.Y.; Tao, X.Q.; Wang, H.S. A novel hydrogen peroxide sensor based on the direct electron transfer of horseradish peroxidase immobilized on silica-hydroxyapatite hybrid film. Biosens. Bioelectron. 2009, 24, 1141-1145.

1054. Lu, L.; Zhang, L.; Zhang, X.; Huan, S.; Shen, G.; Yu, R. A novel tyrosinase biosensor based on hydroxyapatite-chitosan nanocomposite for the detection of phenolic compounds. Anal. Chim. Acta 2010, 665, 146-151.

1055. Wang, S.; Lei, Y.; Zhang, Y.; Tang, J.; Shen, G.; Yu, R. Hydroxyapatite nanoarray-based cyanide biosensor. Anal. Biochem. 2010, 398, 191-197.

1056. Tagaya, M.; Ikoma, T.; Migita, S.; Okuda, M.; Takemura, T.; Hanagata, N.; Yoshioka, T.; Tanaka, J. Fetal bovine serum adsorption onto hydroxyapatite sensor monitoring by quartz crystal microbalance with dissipation technique. Mater. Sci. Eng. B 2010, 173, 176-181.

1057. Wypych, G. Handbook of Fillers, 3rd ed.; ChemTec Publishing: New York, NY, USA, 2009; p. 800.

1058. Almora-Barrios, N.; de Leeuw, N.H. A density functional theory study of the interaction of collagen peptides with hydroxyapatite surfaces. Langmuir 2010, 26, 14535-14542.

1059. Zhang, H.P.; Lu, X.; Leng, Y.; Fang, L.; Qu, S.; Feng, B.; Weng, J.; Wang, J. Molecular dynamics simulations on the interaction between polymers and hydroxyapatite with and without coupling agents. Acta Biomater. 2009, 5, 1169-1181.

1060. Rhee, S.H.; Lee, J.D.; Tanaka, J. Nucleation of hydroxyapatite crystal through chemical interaction with collagen. J. Am. Ceram. Soc. 2000, 83, 2890-2892.

1061. Lin, X.; Li, X.; Fan, H.; Wen, X.; Lu, J.; Zhang, X. In situ synthesis of bone-like apatite/collagen nano-composite at low temperature. Mater. Lett. 2004, 58, 3569-3572.

1062. Zhang, W.; Liao, S.S.; Cui, F.Z. Hierarchical self-assembly of nanofibrils in mineralized collagen. Chem. Mater. 2003, 15, 3221-3226.

1063. Liu, Q.; de Wijn, J.R.; van Blitterswijk, C.A. Covalent bonding of PMMA, PBMA and poly(HEMA) to hydroxyapatite particles. J. Biomed. Mater. Res. 1998, 40, 257-263.

1064. Li, J.; Chen, Y.P.; Yin, Y.; Yao, F.; Yao, K. Modulation of nano-hydroxyapatite size via formation on chitosan-gelatin network film in situ. Biomaterials 2007, 28, 781-790.

1065. Zhou, S.; Zheng, X.; Yu, X.; Wang, J.; Weng, J.; Li, X.; Feng, B.; Yin, M. Hydrogen bonding interaction of poly(D,L-lactide)/hydroxyapatite nanocomposites. Chem. Mater. 2007, 19, 247-253.

1066. Ficai, A.; Andronescu, E.; Ghitulica, C.; Voicu, G.; Trandafir, V.; Manzu, D.; Ficai, M.; Pall, S. Colagen/hydroxyapatite interactions in composite biomaterials. Materiale Plastice 2009, 46, 11-15.

1067. Li, J.; Dou, Y.; Yang, J.; Yin, Y.; Zhang, H.; Yao, F.; Wang, H.; Yao, K. Surface characterization and biocompatibility of micro- and nano-hydroxyapatite/chitosan-gelatin network films. Mater. Sci. Eng. C 2009, 29, 1207-1215.

1068. Danilchenko, S.N.; Kalinkevich, O.V.; Kuznetsov, V.N.; Kalinkevich, A.N.; Kalinichenko, T.G.; Poddubny, I.N.; Starikov, V.V.; Sklyar, A.M.; Sukhodub, L.F. Thermal transformations of the mineral component of composite biomaterials based on chitosan and apatite. Crystal Res. Technol. 2010, 45, 685-691. 
1069. Popescu, L.M.; Rusti, C.F.; Piticescu, R.M.; Buruiana, T.; Valero, T.; Kintzios, S. Synthesis and characterization of acid polyurethane-hydroxyapatite composites for biomedical applications. J. Compos. Mater. 2013, 47, 603-612.

1070. Boanini, E.; Gazzano, M.; Rubini, K.; Bigi, A. Composite nanocrystals provide new insight on alendronate interaction with hydroxyapatite structure. Adv. Mater. 2007, 19, 2499-2502.

1071. Tjandra, W.; Yao, J.; Ravi, P.; Tam, K.C.; Alamsjah, A. Nanotemplating of calcium phosphate using a double-hydrophilic block copolymer. Chem. Mater. 2005, 17, 4865-4872.

1072. Tsuchiya, K.; Yoshioka, T.; Ikoma, T.; Tanaka, J. Chemical interaction between hydroxyapatite and organic molecules in biomaterials. Ceram. Trans. 2010, 210, 531-535.

1073. Grossman, R.F. Coupling Agents. In Polymer modifiers and additives; Lutz, J.T., Jr., Grossman, R.F., Eds.: CRC Press: Boca Raton, FL, USA, 2000; pp. 95-106.

1074. Chang, M.C.; Ikoma, T.; Kikuchi, M.; Tanaka, J. Preparation of a porous hydroxyapatite/collagen nanocomposite using glutataldehyde as a crosslinkage agent. J. Mater. Sci. Lett. 2001, 20, 11991201.

1075. Sousa, R.A.; Reis, R.L.; Cunha, A.M.; Bevis, M.J. Coupling of HDPE/hydroxyapatite composites by silane-based methodologies. J. Mater. Sci. Mater. Med. 2003, 14, 475-487.

1076. Dupraz, A.M.P.; de Wijn, J.R.; van der Meer, S.A.T.; Goedemoed, J.H. Biocompatibility screening of silane-treated hydroxyapatite powders for use as filler in resorbable composites. $J$. Mater. Sci. Mater. Med. 1996, 7, 731-738.

1077. Dupraz, A.M.P.; de Wijn, J.R.; van der Meer, S.A.T.; de Groot, K. Characterization of silanetreated hydroxyapatite powders reinforced for use as filler in biodegradable composites. $J$. Biomed. Mater. Res. 1996, 30, 231-238.

1078. Liao, J.G.; Wang, X.J.; Zuo, Y.; Zhang, L.; Wen, J.Q.; Li, Y. Surface modification of nanohydroxyapatite with silane agent. J. Inorg. Mater. 2008, 23, 145-149.

1079. Rakmae, S.; Ruksakulpiwat, Y.; Sutapun, W.; Suppakarn, N. Physical properties and cytotoxicity of surface-modified bovine bone-based hydroxyapatite/poly(lactic acid) composites. J. Compos. Mater. 2011, 45, 1259-1269.

1080. Rakmae, S.; Ruksakulpiwat, Y.; Sutapun, W.; Suppakarn, N. Effect of silane coupling agent treated bovine bone based carbonated hydroxyapatite on in vitro degradation behavior and bioactivity of PLA composites. Mater. Sci. Eng. C 2012, 32, 1428-1436.

1081. Carmen, A.; Rosestela, P.; Arquimedes, K.; Gema, G.; Nohemy, D.; Yanixia, S.; Luís, B.J. Characterization of HDPE/HA composites treated with titanate and zirconate coupling agents. Macromol. Symp. 2007, 247, 190-198.

1082. Misra, D.N. Adsorption of zirconyl salts and their acids on hydroxyapatite: use of salts as coupling agents to dental polymer composites. J. Dent. Res. 1985, 12, 1405-1408.

1083. Chow, W.S.; Tham, W.L.; Ishak, Z.A.M. Improvement of microstructure and properties of poly(methyl methacrylate)/hydroxyapatite composites treated with zirconate coupling agent. $J$. Thermoplast. Compos. Mater. 2012, 25, 165-180.

1084. Tham, W.L.; Chow, W.S.; Ishak, Z.A.M. Effects of titanate coupling agent on the mechanical, thermal, and morphological properties of poly(methyl methacrylate)/hydroxyapatite denture base composites. J. Compos. Mater. 2011, 45, 2335-2345. 
1085. Shen, D.; Fang, L.; Chen, X.; Tang, Y. Structure and properties of polyacrylic acid modified hydroxyapatite/liquid crystal polymer composite. J. Reinfor. Plast. Comp. 2011, 30, 1155-1163.

1086. Liu, Q.; de Wijn, J.R.; van Blitterswijk, C.A. A study on the grafting reaction of isocyanates with hydroxyapatite particles. J. Biomed. Mater. Res. 1998, 40, 358-364.

1087. Tanaka, H.; Yasukawa, A.; Kandori, K.; Ishikawa, T. Surface modification of calcium hydroxyapatite with hexyl and decyl phosphates. Colloids Surf. A 1997, 125, 53-62.

1088. Tanaka, H.; Watanabe, T.; Chikazawa, M.; Kandori, K.; Ishikawa, T. TPD, FTIR, and molecular adsorption studies of calcium hydroxyapatite surface modified with hexanoic and decanoic acids J. Colloid Interf. Sci. 1999, 214, 31-37.

1089. Borum-Nicholas, L.; Wilson, O.C., Jr. Surface modification of hydroxyapatite. Part I. Dodecyl alcohol. Biomaterials 2003, 24, 3671-3679.

1090. Borum, L.; Wilson, O.C., Jr. Surface modification of hydroxyapatite. Part II. Silica. Biomaterials 2003, 24, 3681-3688.

1091. Li, Y.; Weng, W. Surface modification of hydroxyapatite by stearic acid: Characterization and in vitro behaviors. J. Mater. Sci. Mater. Med. 2008, 19, 19-25.

1092. Lee, S.C.; Choi, H.W.; Lee, H.J.; Kim, K.J.; Chang, J.H.; Kim, S.Y.; Choi, J.; Oh, K.S.; Jeong, Y.K. In-situ synthesis of reactive hydroxyapatite nano-crystals for a novel approach of surface grafting polymerization. J. Mater. Chem. 2007, 17, 174-180.

1093. Sánchez-Salcedo, S.; Colilla, M.; Izquierdo-Barba, I.; Vallet-Regi, M. Design and preparation of biocompatible zwitterionic hydroxyapatite. J. Mater. Chem. B 2013, 1, 1595-1606.

1094. Morita, S.; Furuya, K.; Ishihara, K.; Nakabayashi, N. Performance of adhesive bone cement containing hydroxyapatite particles. Biomaterials 1998, 19, 1601-1606.

1095. Shinzato, S.; Nakamura, T.; Tamura, J.; Kokubo, T.; Kitamura, Y. Bioactive bone cement: Effects of phosphoric ester monomer on mechanical properties and osteoconductivity. J. Biomed. Mater. Res. 2001, 56, 571-577.

1096. Dorozhkin, S.V. Is there a chemical interaction between calcium phosphates and hydroxypropylmethylcellulose (HPMC) in organic/inorganic composites? J. Biomed. Mater. Res. 2001, 54, 247-255.

1097. Omori, M.; Okubo, A.; Otsubo, M.; Hashida, T.; Tohji, K. Consolidation of multi-walled carbon nanotube and hydroxyapatite coating by the spark plasma system (SPS). Key Eng. Mater. 2004, 254-256, 395-398.

1098. Zhao, B.; Hu, H.; Mandal, S.K.; Haddon, R.C. A bone mimic based on the self-assembly of hydroxyapatite on chemically functionalized single-walled carbon nanotubes. Chem. Mater. 2005, 17, 3235-3241.

1099. Aminzare, M.; Eskandari, A.; Baroonian, M.H.; Berenov, A.; Hesabi, Z.R.; Taheri, M.; Sadrnezhaad, S.K. Hydroxyapatite nanocomposites: Synthesis, sintering and mechanical properties. Ceram. Int. 2013, 39, 2197-2206.

1100. Kasuga, T.; Yoshida, M.; Ikushima, A.J.; Tuchiya, M.; Kusakari, H. Bioactivity of zirconia-toughened glass-ceramics. J. Am. Ceram. Soc. 1992, 75, 1884-1888.

1101. Ehrenfried, L.M.; Patel, M.H.; Cameron, R.E. The effect of tri-calcium phosphate (TCP) addition on the degradation of polylactide-co-glycolide (PLGA). J. Mater. Sci. Mater. Med. 2008, 19, 459-466. 
1102. Ehrenfried, L.M.; Farrar, D.; Cameron, R.E. Degradation properties of co-continuous calciumphosphate-polyester composites. Biomacromolecules 2009, 10, 1976-1985.

1103. Pan, J.; Han, X.; Niu, W.; Cameron, R.E. A model for biodegradation of composite materials made of polyesters and tricalcium phosphates. Biomaterials 2011, 32, 2248-2255.

1104. Barrett, C.E.; Cameron, R.E. X-ray microtomographic analysis of $\alpha$-tricalcium phosphatepoly(lactic-co-glycolic) acid nanocomposite degradation. Polymer 2014, 55, 4041-4049.

1105. Heidemann, W.; Jeschkeit, S.; Ruffieux, K.; Fischer, J.H.; Wagner, M.; Krüger, G.; Wintermantel, E.; Gerlach, K.L. Degradation of poly(D,L)lactide implants with or without addition of calciumphosphates in vivo. Biomaterials 2001, 22, 2371-2381.

1106. Adamus, A.; Jozwiakowska, J.; Wach, R.A.; Suarez-Sandoval, D.; Ruffieux, K. Rosiak, J.M. In vitro degradation of $\beta$-tricalcium phosphate reinforced poly(L-lactic acid). Mater. Sci. Forum 2012, 714, 283-290.

1107. Ahola, N.; Veiranto, M.; Rich, J.; Efimov, A.; Hannula, M.; Seppälä, J.; Kellomäki, M. Hydrolytic degradation of composites of poly(L-lactide-co-e-caprolactone) $70 / 30$ and $\beta$-tricalcium phosphate. J. Biomater. Appl. 2013, 28, 529-543.

1108. Dorozhkin, S.V. Inorganic chemistry of the dissolution phenomenon: The dissolution mechanism of calcium apatites at the atomic (ionic) level. Comments Inorg. Chem. 1999, 20, 285-299.

1109. Dorozhkin, S.V. Dissolution mechanism of calcium apatites in acids: a review of literature. World J. Methodol. 2012, 2, 1-17.

1110. Furukawa, T.; Matsusue, Y.; Yasunaga, T.; Shikinami, Y.; Okuno, M.; Nakamura, T. Biodegradation behavior of ultra-high strength hydroxyapatite/poly(L-lactide) composite rods for internal fixation of bone fractures. Biomaterials 2000, 21, 889-898.

1111. Furukawa, T.; Matsusue, Y.; Yasunaga, T.; Nakagawa, Y.; Okada, Y.; Shikinami, Y.; Okuno, M.; Nakamura, T. Histomorphometric study on high-strength hydroxyapatite/poly(L-lactide) composite rods for internal fixation of bone fractures. J. Biomed. Mater. Res. 2000, 50, 410-419.

1112. Yasunaga, T.; Matsusue, Y.; Furukawa, T.; Shikinami, Y.; Okuno, M.; Nakamura, T. Bonding behaviour of ultrahigh strength unsintered hydroxyapatite particles/poly(L-lactide) composites to surface of tibial cortex in rabbits. J. Biomed. Mater. Res. 1999, 47, 412-419.

1113. Marques, A.P.; Reis, R.L.; Hunt, J.A. In vitro evaluation of the biocompatibility of novel starch based polymeric and composite material. Biomaterials 2002, 6, 1471-1478.

1114. Mendes, S.C.; Bovell, Y.P.; Reis, R.L.; Cunha, A.M.; de Bruijn, J.D.; van Blitterswijk, C.A. Biocompatibility testing of novel starch-based materials with potential application in orthopaedic surgery. Biomaterials 2001, 22, 2057-2064.

1115. Habraken, W.J.E.M.; Liao, H.B.; Zhang. Z.; Wolke, J.G.C.; Grijpma, D.W.; Mikos, A.G.; Feijen, J.; Jansen, J.A. In vivo degradation of calcium phosphate cement incorporated into biodegradable microspheres. Acta Biomater. 2010, 6, 2200-2211.

1116. Ngiam, M.; Liao, S.; Patil, A.J.; Cheng, Z.; Chan, C.K.; Ramakrishna, S. The fabrication of nano-hydroxyapatite on PLGA and PLGA/collagen nanofibrous composite scaffolds and their effects in osteoblastic behavior for bone tissue engineering. Bone 2009, 45, 4-16.

1117. Hasegawa, S.; Ishii, S.; Tamura, J.; Furukawa, T.; Neo, M.; Matsusue, Y.; Shikinami, Y.; Okuno, M.; Nakamura, T. A 5-7 year in vivo study of high-strength hydroxyapatite/poly(L-lactide) composite rods for the internal fixation of bone fractures. Biomaterials 2006, 27, 1327-1332. 
1118. Bongio, M.; van den Beucken, J.J.J.P.; Leeuwenburgh, S.C.G.; Jansen, J.A. Development of bone substitute materials: From 'biocompatible' to 'instructive'. J. Mater. Chem. 2010, 20, 8747-8759.

1119. Meyers, M.A.; Lin, A.Y.M.; Seki, Y.; Chen, P.Y.; Kad, B.K.; Bodde, S. Structural biological composites: An overview. JOM 2006, 58, 36-43.

(C) 2015 by the author; licensee MDPI, Basel, Switzerland. This article is an open access article distributed under the terms and conditions of the Creative Commons Attribution license (http://creativecommons.org/licenses/by/4.0/). 